\title{
A FLEXIBLE HIERARCHICAL MODEL-BASED CONTROL METHODOLOGY FOR VEHICLE ACTIVE SAFETY SYSTEMS
}

\author{
by
}

\section{Sehyun Chang}

A dissertation submitted in partial fulfillment of the requirements for the degree of

Doctor of Philosophy

(Mechanical Engineering)

in The University of Michigan

2007

Doctoral Committee:

Professor Timothy J. Gordon, Chair

Professor Huei Peng

Professor A.Galip Ulsoy

Associate Professor Jing Sun 
To my family 


\section{ACKNOWLEDGMENTS}

I would like to especially thank my advisor, Prof. Dr. Timothy J. Gordon, for his support, encouragement, and confidence during my doctorial studies. He provided great inspiration and gentle guidance, both of which encouraged me to pursue my interests throughout my research. I also would like to express my deep appreciation to the other members of my committee, Prof. Galip Ulsoy, Prof. Huei Peng and Prof. Jing Sun, for their advice and comments, which helped guide the direction of my work. To Prof. Dr. Taehyun Shim at the University of Michigan-Dearborn, I express my thanks for his insights into vehicle dynamics and control systems.

I gratefully acknowledge the support of Hyundai Motor Company and KIA Motors Corporation in the form of a scholarship for my study. I also appreciate University of Michigan Transportation Research Institute (UMTRI) giving me the opportunity to conduct my dissertation research in UMTRI.

I do not want to forget my colleagues and friends in the Automotive Laboratory whose friendship and inspiring ideas were a great source of encouragement. I can not imagine completing this work without Dr. Dae-Kyun Kim, Dr. Min Joong Kim, Dr. Ashish Deshpande, Jeong-Seok Kim, Youseok Kou, Dongsoo Kang, Jing Zhou, Jinming Liu, Yongsong Chen, Dongsuk Kum, Hsin-Hsiang Yang, and Seok Lee. I also want to thank my colleagues, Keychun Park, Youngjae Kim, and Seungjun Shin, who came together with me from Hyundai Motor Company to Michigan.

Finally, I would like to express my loving appreciation to my wife, Myounghee Lee, my daughter, Yuji, and my son, Yeonje (Phillip), for their love and patience. 


\section{TABLE OF CONTENTS}

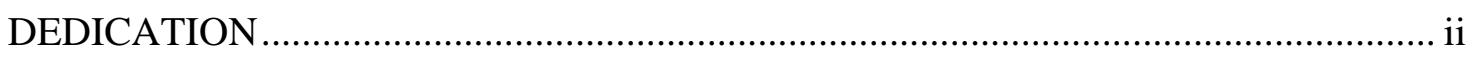

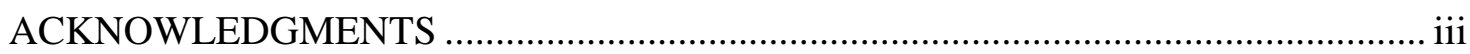

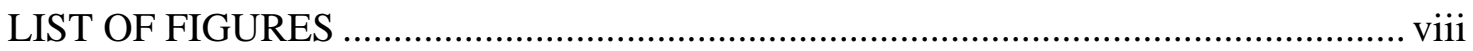

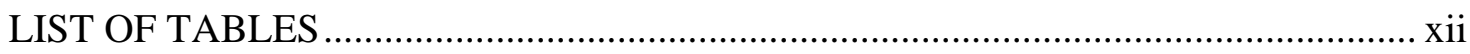

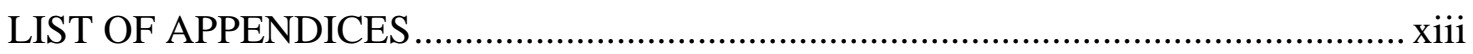

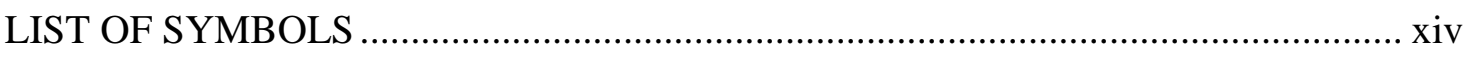

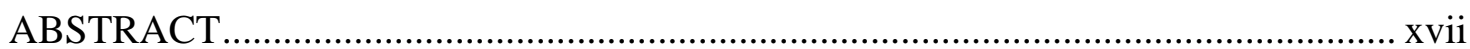

\section{CHAPTER}

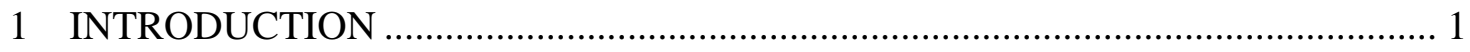

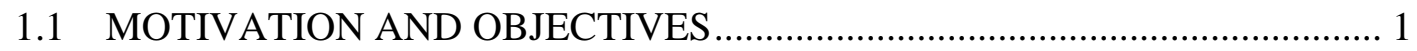

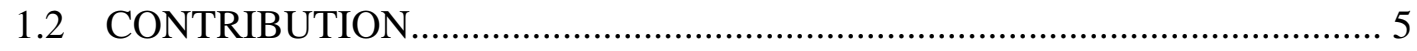

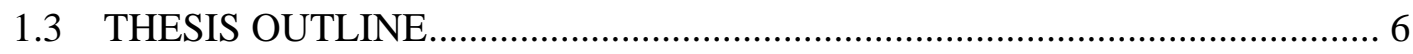

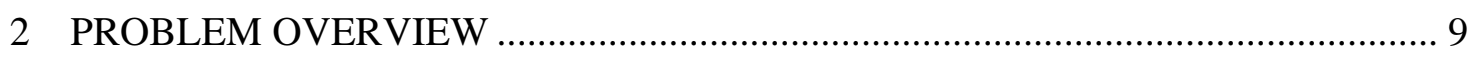

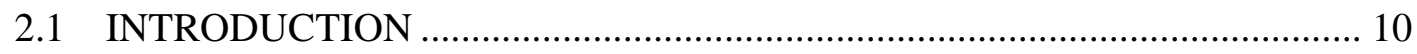

2.1.1 Why an integrated vehicle control system? ........................................ 10

2.1.2 What are the intended benefits of an Integrated vehicle control

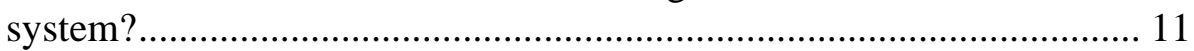

2.2 INTEGRATED VEHICLE CONTROL APPLICATIONS ............................... 11

2.3 INTEGRATED VEHICLE CONTROL ARCHITECTURE.............................. 13

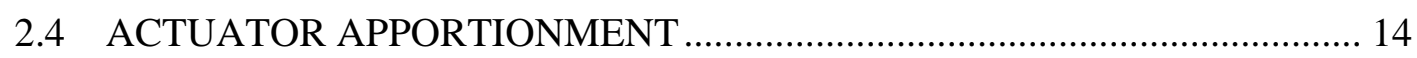

2.4.1 Comparision of the actuator potential for yaw moment generation ...... 15

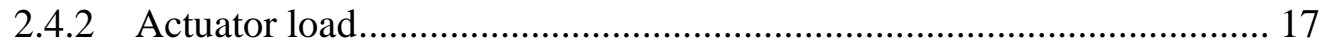

2.5 MODEL PREDICTIVE CONTROL REVIEW................................................ 19

2.5.1 Why should MPC be used in integrated chassis control? ..................... 19

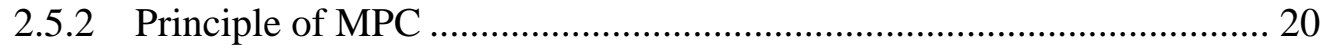

2.5.3 Approaches to MPC implementation...................................................... 22

2.5.4 Model formulation ............................................................................ 23 


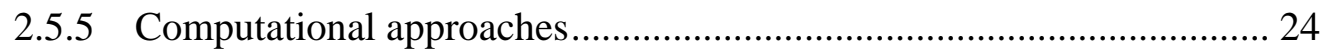

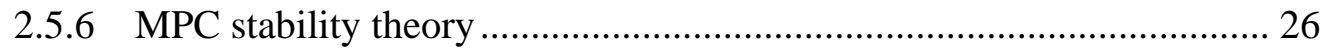

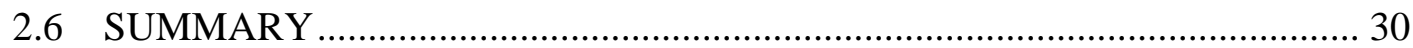

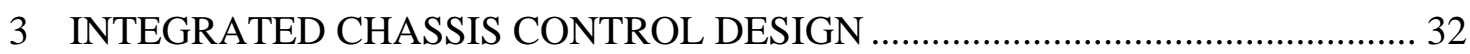

3.1 OVERALL CONTROLLER STRUCTURE …................................................ 33

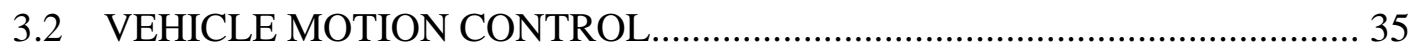

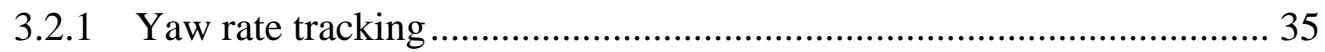

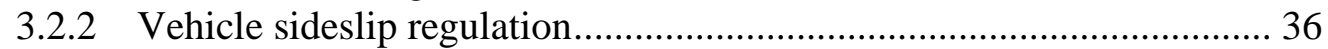

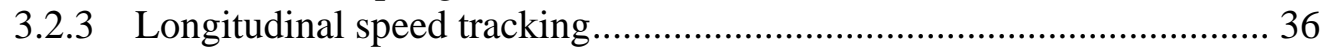

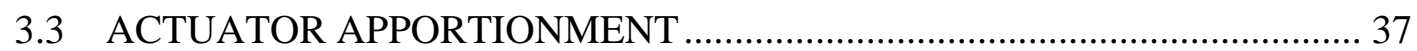

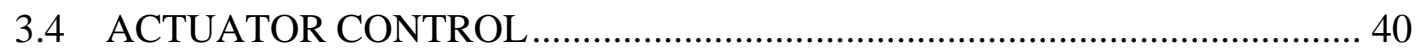

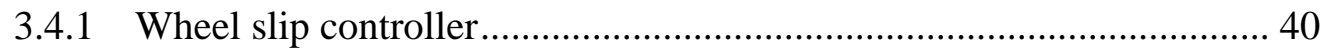

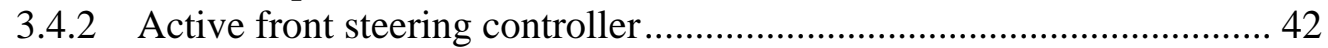

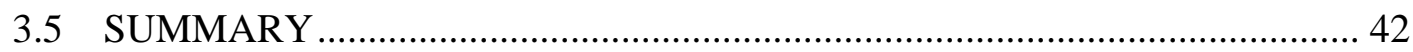

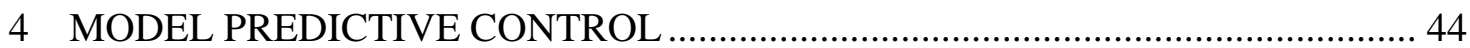

4.1 LINEAR TIME-VARYING MPC DESIGN ................................................. 44

4.1.1 Difference equations at non-equilibrium points .................................. 45

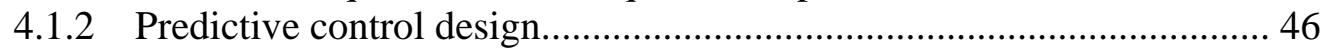

4.2 IMPLEMENTATION OF MPC ON VEHICLE CONTROL SYSTEMS ......... 50

4.2.1 Degree of Freedom of vehicle model................................................. 50

4.2.2 Weighting parameters in the cost function ........................................ 52

4.2.3 Prediction horizon and sample time of MPC ...................................... 53

4.2.4 Actuator constraints ............................................................................ 55

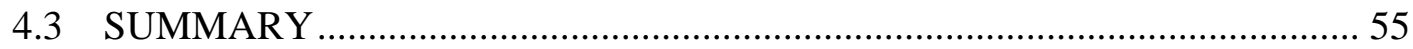

5 PERFORMANCE BENEFITS OF OPTIMAL ACTUATOR

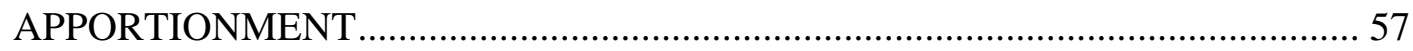

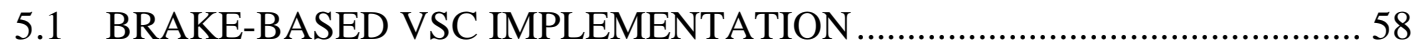

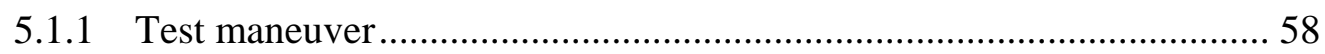

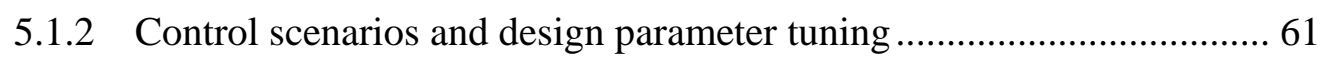

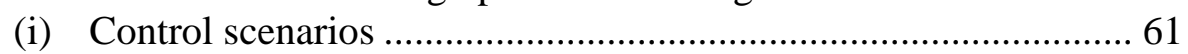

(ii) Control design parameter tuning ................................................... 62

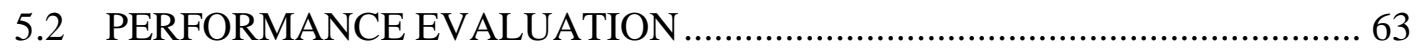

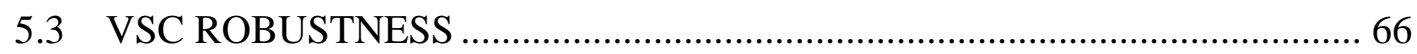

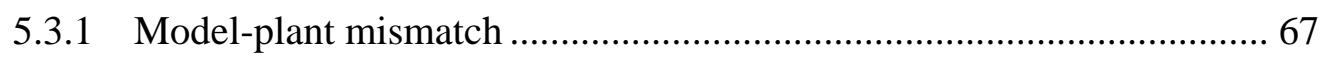

(i) Controller design parameter tuning ............................................. 67

(ii) Performance comparison between Retuned-weight LTV-

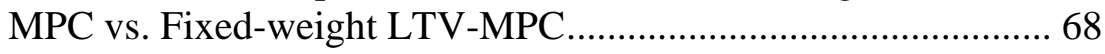

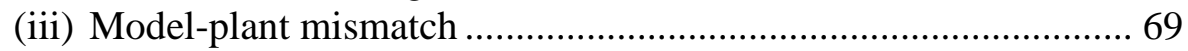

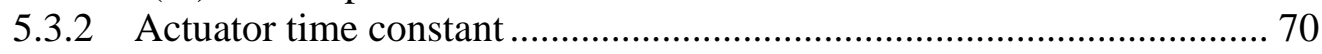




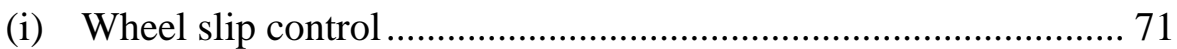

(ii) Brake-based VSC........................................................................ 73

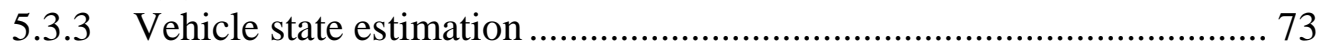

(i) Vehicle state estimation results................................................... 75

(ii) Performance comparison of the VSC between exact measurement vs. EKF ............................................................. 77

5.3.4 Road surface friction coefficient adaptation .......................................... 78

(i) VSC performance without road surface friction adaptation .......... 78

(ii) VSC performance with road surface friction adaptation .............. 80

(iii) Road surface friction estimation: .................................................... 82

5.4 MORE COMPLEX DRIVING MANEUVERS …......................................... 83

5.4.1 Open-loop brake-in-turn maneuver........................................................ 83

5.4.2 Closed-loop Lane change maneuver ..................................................... 86

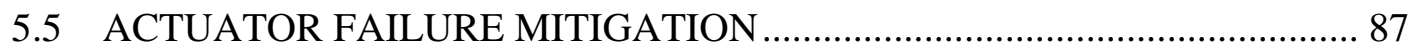

5.6 FEASIBILITY OF REAL-TIME IMPLEMENTATION ….............................. 90

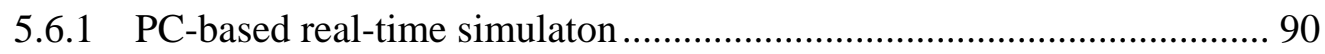

5.6.2 Evaluation of real-time simulation performance ................................. 92

(i) Comparison of controller performance between the real-time QP solver and off-line QP solver ............................................... 92

(ii) Computational load of the real-time QP solver ........................... 93

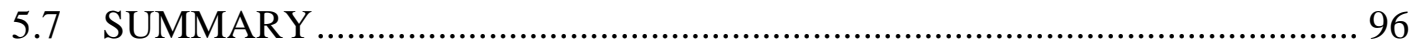

6 CONTROL METHODOLOGY FOR COLLISION AVOIDANCE SYSTEMS ....... 98

6.1 REVIEW OF COLLISION AVOIDANCE SYSTEMS .................................... 99

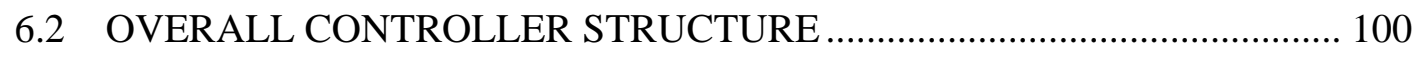

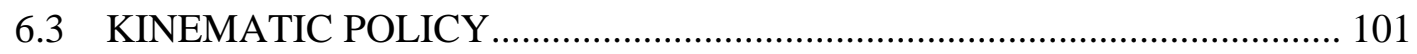

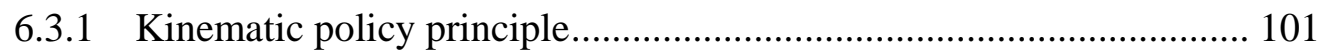

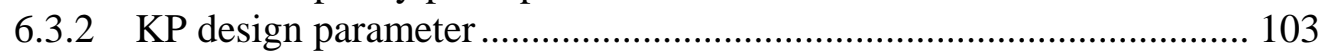

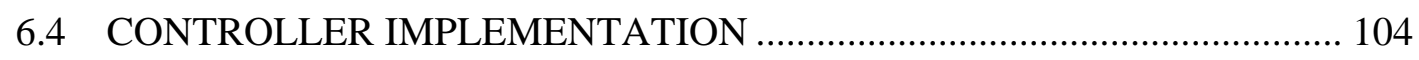

6.4.1 Course layout and vehicle outline..................................................... 105

6.4.2 Vehicle motion critera after lane change ............................................ 105

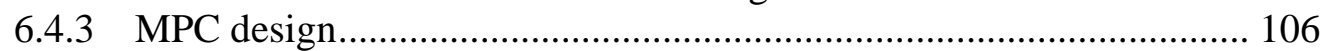

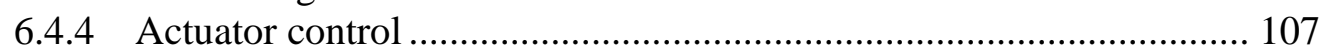

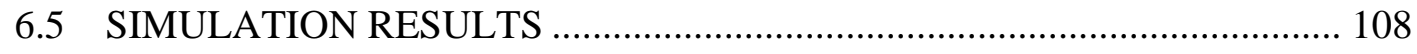

6.5.1 Control performance by an idealized driver ........................................ 108

6.5.2 Controller evaluation for collision avoidance..................................... 109

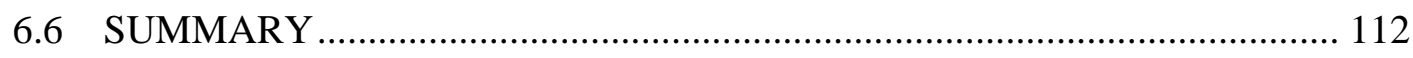

7 CONCLUSIONS AND FUTURE RESEARCH SUGGESTIONS .......................... 114 


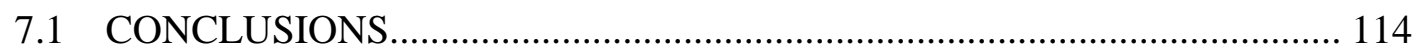

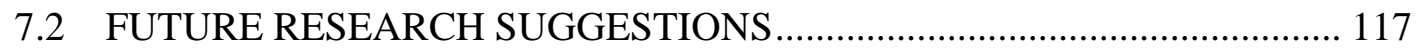

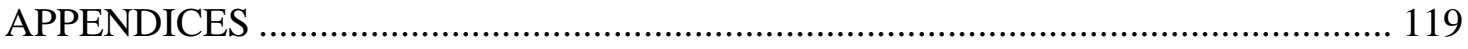

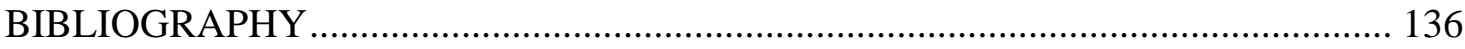




\section{LIST OF FIGURES}

\section{Figure}

Fig. 2.1 Examples of chassis actuators for vehicle control systems 10

Fig. 2.2 Integrated control and the g-g diagram (Tanaka et al., 1992; Gordon et al., 2003)

Fig. 2.3 Potential for yaw moment generation using a nonlinear 8DoF vehicle model ... 16

Fig. 2.4 Yaw moment comparison of the rule-based actuator apportionment and the

flexible actuator apportionment for sine maneuver.

Fig. 2.5 Vehicle responses comparison of the rule-based actuator apportionment and the flexible actuator apportionment for sinusoidal steering maneuver.....

Fig. 2.6 Model Predictive Control concept

Fig. 2.7 Exponential decay of the state trajectory (de Oliveira Kothare and Morari, 2000)

Fig. 3.1 Hierarchical control architecture of flexible integrated chassis control

Fig. 4.1 Comparison of a nonlinear model and a LTV-model at instant 1.4s during a sine with dwell test (8DoF nonlinear vehicle model and no braking)

Fig. 5.1 Over-steer vehicle response during emergency lane change maneuver on high friction road - Arrow represents a conceptual lateral tire force at a front axle (Hac and Bodie, 2002)

Fig. 5.2 Sine-With-Dwell maneuver steering wheel position and yaw velocity information (NHTSA, 2007).

Fig. 5.3 Performance comparison for Sine-With-Dwell maneuver (8DoF, $\left.U_{0}=80 \mathrm{~km} / \mathrm{h}, \mu_{H}=0.9\right)$

Fig. 5.4 Yaw rate and sideslip responses during Sine-With-Dwell maneuver (8DoF, $U_{0}=80 \mathrm{~km} / \mathrm{h}, \mu_{H}=0.9$ ) 64

Fig. 5.5 Slip ratio responses (a-c) and braking torque responses (d-f) according to different controllers during Sine-With-Dwell maneuver (8DoF, $\left.U_{0}=80 \mathrm{~km} / \mathrm{h}, \mu_{H}=0.9\right)$ 65 
Fig. 5.6 Vehicle trajectories and moving distances $(d)$ for Sine-With-Dwell maneuver (8DoF, $U_{0}=80 \mathrm{~km} / \mathrm{h}, \mu_{H}=0.9$ )......

Fig. 5.7 Retuned weighs of cost function for different driving conditions w.r.t. road surface friction and initial vehicle speed (8 DoF)

Fig. 5.8 Performance metrics of (a) retuned weights of cost function and (b) fixed weights of cost function for the exact model-plant match (8DoF).

Fig. 5.9 Comparison between CarSim and 8 DoF tire models

Fig. 5.10 Performance metrics of (a) retuned weights of cost function and (b) fixed weights of cost function for the model-plant mismatch (CarSim).

Fig. 5.11 Block diagram of actuator time constant test using wheel slip controller.

Fig. 5.12 Comparisons of total tracking error for target slip ratio with varying brake time constants $\left(\lambda_{d, f}=-0.12, \lambda_{d, r}=-0.1, \mu_{H}=0.9,8 \mathrm{DoF}\right)$

Fig. 5.13 Comparisons of target slip ratio tracking with varying brake time constants $\left(\lambda_{d, f}=-0.12, \lambda_{d, r}=-0.1, \mu_{H}=0.9,8 \mathrm{DoF}\right)$

Fig. 5.14 Performance summary of the fixed-weight LTV-MPC VSC for different brake time constants in terms of mean and standard deviation of 9 different driving conditions

Fig. 5.15 Vehicle longitudinal and lateral speed estimation using EKF during SineWith-Dwell maneuver of LTV-MPC VSC (CarSim, $\left.U_{0}=80 \mathrm{~km} / \mathrm{h}, \mu_{H}=0.9\right)$......... 76

Fig. 5.16 Longitudinal tire force estimation using EKF during Sine-With-Dwell maneuver of LTV-MPC VSC (CarSim, $U_{0}=80 \mathrm{~km} / \mathrm{h}, \mu_{H}=0.9$ ).

Fig. 5.17 Performance metrics of (a) the fixed-weight LTV-MPC with no noise and known state assumption and (b) the fixed-weight LTV-MPC with EKF estimation during Sine-With-Dwell maneuver

Fig. 5.18 Overall procedure of road surface friction estimation.

Fig. 5.19 Performance metrics of (a) exact known road surface friction for the controller and (b) model-plant mismatch (Controller: $\mu_{H}=0.9$, Plant: various $\mu_{H}$ ) during Sine-With-Dwell maneuver

Fig. 5.20 Flow chart of road surface friction estimation algorithm 80

Fig. 5.21 Examples of road surface friction estimation during Sine-With-Dwell maneuver under three different driving conditions (CarSim and EKF estimation).. 82 
Fig. 5.22 Performance metrics of the road surface friction adaptation using the fixedweight LTV MPC during Sine-With-Dwell maneuver (CarSim and EKF estimation).

Fig. 5.23 Vehicle responses during the open-loop brake-in-turn maneuver (CarSim and EKF estimation. $U_{0}=60 \mathrm{~km} / \mathrm{h}, \mu_{H}=0.3, \delta_{H W}=180 \mathrm{deg}$ ).

Fig. 5.24 Vehicle trajectories during the open-loop brake-in-turn maneuver (CarSim and EKF estimation. $\left.U_{0}=60 \mathrm{~km} / \mathrm{h}, \mu_{H}=0.3, \delta_{H W}=180 \mathrm{deg}\right)$.

Fig. 5.25 Performance metrics of the ABS controller during an open-loop brake-inturn maneuver $\delta_{H W}=180 \mathrm{deg}$ (CarSim and EKF estimation).....

Fig. 5.26 Performance metrics of the FICC controller during an open-loop brake-inturn maneuver $\delta_{H W}=180 \mathrm{deg}$ (CarSim and EKF estimation).

Fig. 5.27 Performance comparison during the closed-loop lane change maneuver with an ideal driver (CarSim and EKF estimation, $U_{0}=60 \mathrm{~km} / \mathrm{h}, \mu_{H}=0.3$ ).

Fig. 5.28 Vehicle trajectories and handwheel steer inputs during the closed-loop lane change maneuver with an ideal driver (CarSim and EKF estimation. $U_{0}=60 \mathrm{~km} / \mathrm{h}, \mu_{H}=0.3$ )

Fig. 5.29 Vehicle trajectory during open-loop straight maneuver with full braking and front left wheel braking failure (CarSim).

Fig. 5.30 Vehicle response comparisons during open-loop straight maneuver with full braking and front left wheel braking failure (CarSim)

Fig. 5.31 Handwheel steer response during open-loop straight maneuver with full braking and front left wheel braking failure (CarSim) ....

Fig. 5.32 Performance comparison of the VSC controller using real-time QP solver and Matlab QP solver during Sine-With-Dwell maneuver (8 DoF).

Fig. 5.33 Comparison of vehicle yaw rate and sideslip responses between real-time QP solver and Matlab solver during Sine-With-Dwell maneuver (8 DoF) .............. 91

Fig. 5.34 Simulink block for the evaluation of real-time simulation performance. 93

Fig. 5.35 Mean value of real-time ratio with respect to (a) sample time and $H_{P}$ (fixed $H_{U}=1$ ), (b) $H_{P}$ and $H_{U}$ of the LTV MPC-based VSC (fixed $T_{s}=0.01 \mathrm{~s}$ ), and $H_{P}$ and $H_{U}$ of the LTV MPC-based VSC (fixed $T_{s}=0.002 \mathrm{~s}$ ).

Fig. 5.36 Number of iteration for the QP solver to converge at each instant 96 
Fig. 6.1 (a) Arc-like projection of relative motion between vehicle and external reference point and (b) Simplified geometry of the projected arc

Fig. 6.2 Concepts of compensation time $\tau_{\text {comp }}$ and safety margin radius $\rho_{S F} \ldots \ldots \ldots \ldots . . . . . .103$

Fig. 6.3 Course layout for emergency lane change and vehicle outline ......................... 105

Fig. 6.4 Vehicle motion criteria after lane change ......................................................... 106

Fig. 6.5 Animation frames during emergency lane change - animation time ( 2.9s)

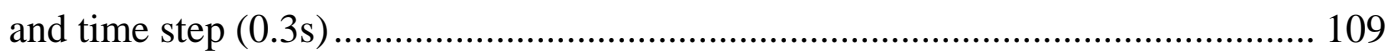

Fig. 6.6 Performance comparison for emergency lane change $\left(d_{o b s}=30 \mathrm{~m}\right)$................. 110

Fig. 6.7 Vehicle trajectories and moving distances $(d)$ for emergency lane change $\left(d_{o b s}=30 \mathrm{~m}\right)$.

Fig. 6.8 Performance comparison for emergency lane change $\left(d_{o b s}=30 \mathrm{~m}\right)$ with a fixed initial speed $U_{0}=87 \mathrm{~km} / \mathrm{h}$

Fig. 6.9 Vehicle trajectories and moving distances $(d)$ for emergency lane change $\left(d_{o b s}=30 \mathrm{~m}\right.$, Initial vehicle speed $\left.=87 \mathrm{~km} / \mathrm{h}\right)$

Fig. 6.10 Vehicle responses comparison for emergency lane change $\left(\mathrm{d}_{\mathrm{obs}}=30 \mathrm{~m}\right.$, Initial vehicle speed $=87 \mathrm{~km} / \mathrm{h}$ )

Fig. A.1 Geometric definition of the 8 DoF vehicle model in the SAE coordinate system

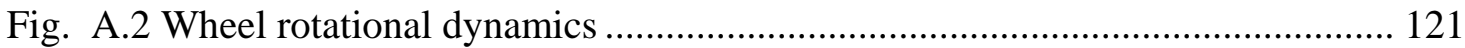

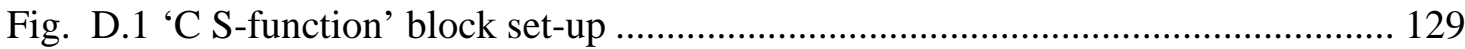

Fig. D.2 Configure the parameters of Simulink solver from the 'Simulation' popup menu in Simulink (Step 1).

Fig. D.3 Configure the parameters of Hardware implementation and Real-time workshop from the 'Simulation' popup menu in Simulink (Step 2)

Fig. D.4 Configure the parameters of signal monitoring from 'Tools' popup menu in Simulink (Step 3)

Fig. D.5 Build a real-time executable model and run simulation from 'Tools' popup menu in Simulink (Step 4)

Fig. E.1 Block diagram of the FICC system. 


\section{LIST OF TABLES}

$\underline{\text { Table }}$

Table 2.1 General classification of MPC approaches ..................................................... 23

Table 4.1 State space components of a predictive vehicle model................................... 51

Table 5.1 Sensor noise characteristics in terms of standard deviation, $\sigma$....................... 74

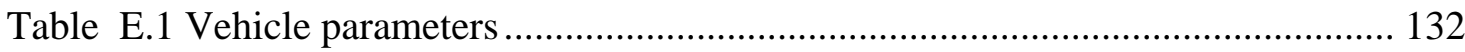

Table E.2 Design parameters of the MPC controller ..................................................... 133

Table E.3 Design parameters of the rule-based controller............................................ 133

Table E.4 List of the inputs and outputs of each module in the FICC system................ 135 


\section{LIST OF APPENDICES}

Appendix

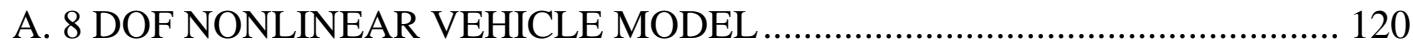

B. SLIDING MODE CONTROL(SMC) FOR WHEEL SLIP CONTROL ............. 123

B.1 Equivalent control ........................................................................... 123

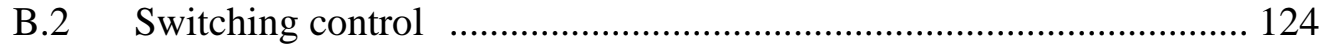

C. RULE-BASED REFENCE CONTROLLER DESIGN ...................................... 127

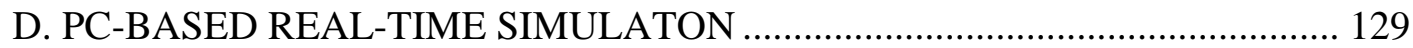

D.1 Configuration of Simulink block, C S-function.................................. 129

D.2 Procedure to run Real-Time Application ............................................ 130

E. LIST OF SIMULATION STUDY PARAMETERS .......................................... 132

E.1 List of vehicle model parameters .................................................. 132

E.2 List of control design parameters................................................... 132

E.3 List of the FICC data flow .............................................................. 134 


\section{LIST OF SYMBOLS}

\begin{tabular}{|c|c|c|}
\hline$m$ & vehicle sprung mass & $(\mathrm{kg})$ \\
\hline g & gravitational acceleration & $\left(\mathrm{m} / \mathrm{s}^{2}\right)$ \\
\hline$I_{x x}$ & roll moment of inertia w.r.t. $\mathrm{x}$-axis & $\left(\mathrm{kg}-\mathrm{m}^{2}\right)$ \\
\hline$I_{z z}$ & yaw moment of inertia w.r.t. z-axis & $\left(\mathrm{kg}-\mathrm{m}^{2}\right)$ \\
\hline$I_{x z}$ & product of inertia w.r.t. $\mathrm{x}$ and $\mathrm{z}$-axis & $\left(\mathrm{kg}-\mathrm{m}^{2}\right)$ \\
\hline$a$ & distance of c.g to front axle & (m) \\
\hline$b$ & distance of c.g to rear axle & (m) \\
\hline$c_{f}, c_{r}$ & half track of front and rear axle & $(\mathrm{m})$ \\
\hline$h_{c g}$ & c.g height above ground & (m) \\
\hline$h_{0}$ & ground to roll axis distance below c.g & (m) \\
\hline$h_{1}$ & distance of $h_{c g}$ to $h_{0}$ & (m) \\
\hline$h_{r f}$ & height of front roll center above ground & $(\mathrm{m})$ \\
\hline$h_{r r}$ & height of rear roll center above ground & (m) \\
\hline$\varepsilon$ & inclined angle between roll axis and $\mathrm{x}$-axis & (deg) \\
\hline$K_{\varphi f}, K_{\varphi r}$ & front and rear roll stiffness & (N-m-s/rad) \\
\hline$K_{p f}, K_{p r}$ & front and rear damping rate & $(\mathrm{N}-\mathrm{m}-\mathrm{s} / \mathrm{rad})$ \\
\hline$I_{w i}$ & $i$-th wheel rotational inertia & $\left(\mathrm{kg}-\mathrm{m}^{2}\right)$ \\
\hline$R_{w}$ & effective wheel rolling radius & $(\mathrm{m})$ \\
\hline$\mu_{H}$ or $\mu_{s}$ & surface friction coefficient & \\
\hline$\tau_{t}$ & tire force build-up time constant & (s) \\
\hline$\tau_{b}$ & brake torque build-up time constant & (s) \\
\hline$\tau_{\mathrm{s}}$ & steering system time constant & (s) \\
\hline$V_{x} / V_{y}$ & longitudinal/lateral velocities of c.g. in body fixed coordinate & $(\mathrm{m} / \mathrm{s})$ \\
\hline$U_{0}$ & initial longitudinal velocity of c.g. & $(\mathrm{m} / \mathrm{s})$ \\
\hline
\end{tabular}




\begin{tabular}{|c|c|c|}
\hline$\phi$ & roll angle & (rad) \\
\hline$p$ & roll angular velocity of c.g. in body fixed coordinate & $(\mathrm{rad} / \mathrm{s})$ \\
\hline$\psi$ & yaw angle & (rad) \\
\hline$r$ or $\dot{\psi}$ & yaw angular velocity of c.g. in body fixed coordinate & $(\mathrm{rad} / \mathrm{s})$ \\
\hline$\dot{r}$ & yaw acceleration of c.g. in body fixed coordinate & $\left(\mathrm{rad} / \mathrm{s}^{2}\right)$ \\
\hline$a_{x}$ & longitudinal acceleration at c.g. & $\left(\mathrm{m} / \mathrm{s}^{2}\right)$ \\
\hline$a_{y}$ & lateral acceleration at c.g. & $\left(\mathrm{m} / \mathrm{s}^{2}\right)$ \\
\hline$S_{x i}$ & longitudinal wheel slip at the $i$-th wheel & \\
\hline$S_{y i}$ & lateral wheel slip at the $i$-th wheel & \\
\hline$\omega_{i}$ & angular velocity of wheel rotation at the $i$-th wheel & $(\mathrm{rad} / \mathrm{s})$ \\
\hline$\delta$ & road wheel steer angle at the front wheel & (rad) \\
\hline$\delta_{H W}$ & peak handwheel steer angle & (rad) \\
\hline$\beta$ & vehicle sideslip angle & $(\mathrm{rad})$ \\
\hline$T_{b i}$ & brake torque at the $i$-th wheel & $(\mathrm{Nm})$ \\
\hline$F_{x i}, F_{y i}$ and $F_{z i}$ & tire forces in vehicle $x, y$ and $\mathrm{z}$ directions at $i$-th tire contact patch & $(\mathrm{N})$ \\
\hline$F_{x w i}$ and $F_{y w i}$ & tire forces in $x$ and $y$ wheel coordinate at $i$-th tire contact patch & $(\mathrm{N})$ \\
\hline$K_{u s}$ & target vehicle under-steer gradient & \\
\hline$r_{d}$ & desired yaw rate & $(\mathrm{rad} / \mathrm{s})$ \\
\hline$r_{\text {threshold }}$ & yaw rate error threshold & $(\mathrm{rad} / \mathrm{s})$ \\
\hline$r_{\text {threshold_percent }}$ & yaw rate error percentage threshold & \\
\hline$\beta_{\text {threshold }}$ & sideslip threshold & (rad) \\
\hline$a_{\text {threshold }}$ & acceleration threshold & $\left(\mathrm{m} / \mathrm{s}^{2}\right)$ \\
\hline$a_{\text {ref }}$ & reference acceleration & $\left(\mathrm{m} / \mathrm{s}^{2}\right)$ \\
\hline$V_{x \text { ref }}$ & reference longitudinal speed & $(\mathrm{m} / \mathrm{s})$ \\
\hline$K_{\text {Paccel }}$ & constant proportional gain of reference longitudinal speed & \\
\hline$H_{P}, H_{U}$ and $H_{W}$ & prediction horizons of outputs $y$, control inputs $u$ and disturbances $w$ & \\
\hline$Q_{V x}, Q_{\beta}$ and $Q_{r}$ & longitudinal speed, sideslip and yaw rate weights of MPC cost functior & \\
\hline$X$ & vehicle $\mathrm{x}$ coordinate & (m) \\
\hline
\end{tabular}


$d_{i}$ relative distance from a vehicle in CAS

$\phi_{i}$

azimuth angle at the $i$ th boundary point in CAS

$\rho_{S F}$

safety radius of CAS

$d_{0 \text { min }}$

minimum distance of CAS

$\tau_{\text {comp }}$

compensation time of CAS 


\begin{abstract}
To improve the stability and safety performance of active safety systems, vehicle control systems are increasingly incorporating sophisticated chassis actuator control functions. Thus vehicle control systems require efficient control algorithms to reduce the amount of duplicate hardware or adopt various actuator combinations in response to diverse customer demands and various hardware combinations from different suppliers. One of the main challenges for efficient controller design is how to create a flexible modular design which can respond to different reference vehicle behaviors and provide optimal actuator apportionment, while avoiding functional conflicts between chassis subsystems.

The proposed control methodology offers a total vehicle motion control solution for an active safety system by addressing the issue of flexible modular design in integrated vehicle control systems. In this thesis, modularity is realized by a model-based hierarchical control structure consisting of three layers: an upper layer for reference vehicle motions, an intermediate layer for actuator apportionment, and a lower layer for stand-alone actuator control. The reference vehicle motions can be determined by any type of reference model for vehicle stability control or collision avoidance control. The actuator apportionment uses Model Predictive Control (MPC) to provide flexibility by balancing tire forces to track target reference vehicle motions while simultaneously considering constrained conditions, such as actuator limits or available actuator combinations. Moreover, the MPC is designed to be feasible in real-time using a linear time-varying MPC approach which avoids the complexity of full nonlinear MPC and addresses the vehicle nonlinearity.
\end{abstract}


The effectiveness of the proposed control structure is investigated for vehicle stability control in terms of handling stability and handling responsiveness. The handing stability and responsiveness of the control structure appear to be robust with respect to various uncertain environments including model-plant mismatch and diverse driving conditions. It is then applied to collision avoidance systems by adapting the reference vehicle motions at the upper level of the controller. Collision avoidance is found to be nearly as effective as that of the combination of an optimizing driver supported by the MPC-based stability controller; this suggests that the MPC approach could be used in future high performance collision avoidance systems. 


\section{CHAPTER 1}

\section{INTRODUCTION}

\subsection{MOTIVATION AND OBJECTIVES}

Vehicle safety devices have been developed to avoid car crashes and minimize harm. These safety devices can be divided into two types: passive systems or active systems. Passive safety systems include such devices as airbags, seatbelt tensioners and other devices designed to protect vehicle occupants when a crash occurs. Active safety systems are designed to maintain vehicle stability or avoid collision using vehicle control systems. Examples of active safety systems are ABS (Anti-locking Braking System), VSC (Vehicle Stability Control) or collision avoidance systems (CAS). Active safety systems are potentially more effective than passive safety systems because the damage due to a crash can be prevented by appropriate control actions. Active safety systems can be represented as vehicle control systems from control viewpoint, which are becoming increasingly refined with advances in hardware.

In the early stages of vehicle control system development, the systems acted to enhance basic actuator performance in the chassis control system to provide special control objectives or special functionalities. For example, ABS was developed to minimize stopping distance, VSC improved yaw stability, and active suspension was introduced for better ride comfort. Other devices were designed for longitudinal, lateral, vertical, yaw and roll motion controls. As the vehicle control systems consisted of more chassis actuator systems to improve vehicle performance, the amount of software (e.g. control logic) and hardware (e.g. sensors or microcomputer) for each actuator system also 
increased. Moreover, automotive manufacturers now face numerous combinations of control systems designed in response to diverse customer demands and various hardware combinations from different suppliers despite having related functionality. This diverse development environment for control systems requires efficient control algorithms which provide ways of reducing duplicate hardware or adopting various actuator combinations in order to save resources (e.g. vehicle cost and human workload). For these reasons, integrated vehicle control as an active safety system has been extensively developed. Integrated vehicle control can improve multiple-objective performance (Ghoneim et al., 2000; Gordon et al., 2003) in terms of safety and comfort, reducing complexity of the control design, reducing system costs and providing design flexibility.

However, to achieve optimal vehicle control in an active safety system, several important issues must be addressed:

\section{Flexible and modular design}

- Vehicle control systems tend to achieve more multiple objectives such as vehicle stability control and collision avoidance control simultaneously as the demands of high vehicle performance increase due to increased safety concern of customers.

- The number of possible combination of available chassis actuators increases due to diverse demands of customer or different automotive suppliers.

- From the control design viewpoint, flexible and modular design become more important to reduce duplicated hardware or adopt various actuator combinations under these demands achieving multiple objectives with increased actuator combination (Gordon et al., 2003)

\section{Controller tuning issues}

- Even though vehicle control systems provide more design freedom to improve vehicle motion control than the mechanistically-based design such as suspension kinematics, vehicle controllers must balance between multiple objectives because 
the emphasis on one objective can cause other objectives to degrade. For example, aggressive handling responsiveness tracking can lead to handling stability problems in terms of vehicle spin-out (Hac et al., 2007).

\section{Real-time implementable controller design from the practical viewpoint}

- Vehicle motion control aims to assist a driver during driver's unfamiliar situations such as severe transient maneuvers by compensating driver's mistake or too late driver's response. This means that control action should be taken on time with fast control decision.

- For optimal actuator apportionment on vehicle control systems or optimal decision approaches in collision avoidance systems, the computational burden of optimization techniques should be addressed for on-line implementation because computational delay causes controller performance to be sacrificed. (Tøndel and Johansen, 2003; Falcone et al., 2007a)

\section{Controller robustness}

- Vehicle control systems are exposed in many uncertain environments such as model-plant mismatch resulting from the difference between a controller and an actual vehicle, unknown road surface friction, measurement noise, vehicle state estimation error, and other vehicle and road conditions.

- Vehicle control systems should be robust under these uncertain driving conditions because safety is a prime goal for vehicle control systems regardless of driving conditions.

\section{Fault detection and isolation (FDI)}

- Fault tolerant control is becoming increasingly essential in automotive chassis safety systems design, for reasons of increased complexity and increased criticality (a single fault causing a major safety system failure). 
- When one of the system components fails due to mechanical or electrical causes, the control performance can not be guaranteed without appropriate fault diagnosis and control adaptation. Thus, it is desirable that a fault should be detected before any hazard is encountered, isolated to identify the source of the fault, and the control system managing the fault condition by adapting and reconfiguring its control logic (J. Yi et al., 2002; Song et al., 2003; Hac, 2006).

\section{Vehicle state estimation}

- Reliable vehicle state estimation is desirable because vehicle control systems need vehicle state information to work appropriately and some of vehicle states are not available by using direct measurements because of measurement hardware limits or cost issues, even though sensors provide more vehicle state information with advances in hardware, for example, the integration of inertial sensors with GPS (Ray, 1997; Ryu et al., 2000; Bevly, 2004; Kiencke and Nielsen, 2005; Wilkin et al., 2005).

\section{Environment information processing}

- Environmental information processing is a critical part, and requires fast and reliable obstacle state estimation (Prakah-Asante el al., 2003). Regarding this obstacle state estimation, intensive research is ongoing and some of its results are already implemented on commercially available vehicles (Takahashi, 2000, Furutani, 2004 and Knoll et al, 2004).

\section{Controller authority policy}

- Controller authority policy is related with the decision or timing for overriding driver inputs using automatic control, and is a critical issue for collision avoidance systems (Seiler et al., 1998; Mai et al., 2000; Hac and Dickinson, 2006; Zhang et al., 2006). 


\subsection{CONTRIBUTION}

Some of important issues mentioned in the previous Section are addressed in this thesis, and the major contributions of this thesis are summarized as follows:

\section{Development of a general hierarchical model-based control methodology for active} safety systems (Chang and Gordon, 2006; Chang and Gordon, 2007a; Chang and Gordon, 2007b)

- The flexible integrated chassis control (FICC) consists of three layers: : (1) vehicle motion control as an upper layer for reference vehicle motions, (2) an intermediate layer for the actuator apportionment, and (3) a lower layer for individual actuator control. The inherent flexibility of the controller comes from the higher layer for reference vehicle motions and the intermediate layer for actuator apportionment.

- The upper layer provides reference motions in terms of desired longitudinal speed, sideslip and yaw rate. In particular, the FICC controller includes the reference motions for vehicle stability control and collision avoidance control as an active safety system.

\section{Flexible actuator apportionment using LTV-MPC}

- MPC is used for the apportionment of available chassis actuators because of its flexibility to handle multi-variables. MPC balances the tracking error and the actuator efficiency, and considers constraint conditions such as physical limit of actuator or vehicle states, simultaneously.

- The proposed MPC is based on the linear time-varying (LTV) MPC. This approach avoids the complexity of full nonlinear MPC and addresses the vehicle nonlinearity by using time-varying linearization at arbitrary (non-equilibrium) operating points. 
- Several strategies for establishing predictive vehicle models of the MPC-based VSC are proposed from the practical implementation viewpoint.

\section{Real-time feasible MPC-based VSC design}

- The LTV-MPC VSC is based on a convex quadratic programming (QP) by using a linearized predictive model. Thus, the pre-existing reliable QP solver is used and the real-time feasibility of the controller is evaluated through a PC-based realtime simulation using Matlab toolbox Real Time Simulation Target.

Collision avoidance controller design (Chang and Gordon, 2007c)

- The proposed control methodology includes both lateral and longitudinal control, extending the functionality of the vehicle stability controller by adopting the reference vehicle motions at the upper level of the controller. The controller performance is evaluated under emergency lane change with full braking.

- The desired vehicle motions are determined using a kinematic policy (KP) for collision avoidance. The KP uses simple information about range and azimuth angles for multiple points that bound the available vehicle trajectory, and prioritizes yaw motion response based on the worst case collision threat.

The more specific contributions are described in Chapter 7.

\subsection{THESIS OUTLINE}

A review of the literature on vehicle control systems is presented in Chapter 2. This review follows the general control architecture of the vehicle control systems described by Gordon et al. (2003), and the role of the actuator apportionment is emphasized in terms of actuator modularity and flexibility, and classified according to two approaches: rule-based apportionment and flexible apportionment. As one solution to realize flexible actuator apportionment in this thesis, a brief review of MPC is 
presented and discusses MPC principles, an overview of MPC approaches, model formulation, computational approaches, and MPC stability theory.

Chapter 3 proposes the flexible integrated chassis control (FICC) as a means to provide a total vehicle control solution in an active safety system, particularly in terms of two control modes: vehicle stability control and collision avoidance control. The FICC is based on a hierarchical control structure consisting of three layers: (1) vehicle motion control as an upper layer for reference vehicle motions, (2) an intermediate layer for the actuator apportionment using Model Predictive Control (MPC) and (3) a lower layer for individual actuator control. The MPC approach in vehicle control systems and stand-alone actuator control are described from practical viewpoints.

Chapter 4 describes the proposed MPC design in detail, and presents several strategies to construct predictive vehicle models for MPC, for example, 2 DoF or $3 \mathrm{DoF}$ nonlinear vehicle models with various actuators for vehicle control systems. Moreover, regarding the MPC design parameter tuning, the weights of the cost function and actuator constrains are discussed.

Chapter 5 investigates the performance benefits of the proposed vehicle stability controller in terms of handling stability and handling responsiveness for a Sine-WithDwell maneuver. The robustness is investigated for various driving conditions by considering model-plant mismatch, estimation effect and other uncertainties. Flexibility is demonstrated by tracking multiple objectives during more complex driving maneuvers, and mitigating actuator failure. A feasibility study of real-time implementation is performed through a PC-based real-time simulation using Matlab toolbox Real Time Simulation target. Finally, we conclude by discussing the dominant factors affecting the controller performance.

In Chapter 6, the proposed controller is implemented in a collision avoidance system and its performance is evaluated under emergency lane change with full braking. The proposed control methodology includes both lateral and longitudinal control, 
extending the functionality of the vehicle stability controller by adapting the reference vehicle motions at the upper level of the controller. The desired vehicle motions in the upper layer are determined using a kinematic policy (KP) for collision avoidance. The KP uses simple information about range and azimuth angles for multiple points that bound the available vehicle trajectory, and prioritizes yaw motion response based on the worst case collision threat.

Finally, Chapter 7 summarizes findings and presents overall conclusions from the study, and outlooks are presented as the subject of ongoing research. 


\section{CHAPTER 2}

\section{PROBLEM OVERVIEW}

This chapter briefly reviews the relevant literature on vehicle control systems which focuses on the general control architecture of the systems, as described by Gordon et al. (2003). The role of the actuator apportionment is emphasized in terms of actuator modularity and flexibility, and classified according to two approaches: rule-based apportionment and flexible apportionment.

As one solution to realize flexible actuator apportionment, model predictive control (MPC) is used to design the proposed controller in this thesis. A brief review of MPC is presented and discusses MPC principles, an overview of MPC approaches, model formulation, computational approaches, and MPC stability theory. A more comprehensive literature review can be found in (Chen and Allgower, 1998; Morari and Lee, 1999; Mayne et al., 2000; Maciejowski, 2002; Allgower et al., 2004) which also examine theoretical MPC limitations such as model uncertainty, stability and robustness analysis.

The expected benefits of integrated vehicle control systems are summarized in Section 2.1. The integrated vehicle control applications and vehicle control architecture are briefly described in Sections 2.2 and 2.3, respectively. Section 2.4 then compares flexible apportionment and rule-based apportionment in terms of yaw moment generation and actuator load on four individual braking control systems. In Section 2.5, a brief review of the MPC is presented as a way to realize the flexible actuator apportionment in this thesis. Finally, Section 2.6 summarizes findings and presents overall conclusions from the study. 


\section{$2.1 \quad$ INTRODUCTION}

\subsubsection{WHY AN INTEGRATED VEHICLE CONTROL SYSTEM?}

In order to assist safe driving, several vehicle control systems have been developed, for example, anti-lock brake systems (ABS), active steering systems, active four-wheel steering control (4WS), traction control(TRC), active suspension systems, active anti-roll bar control systems and other chassis actuators, as shown in Fig. 2.1.

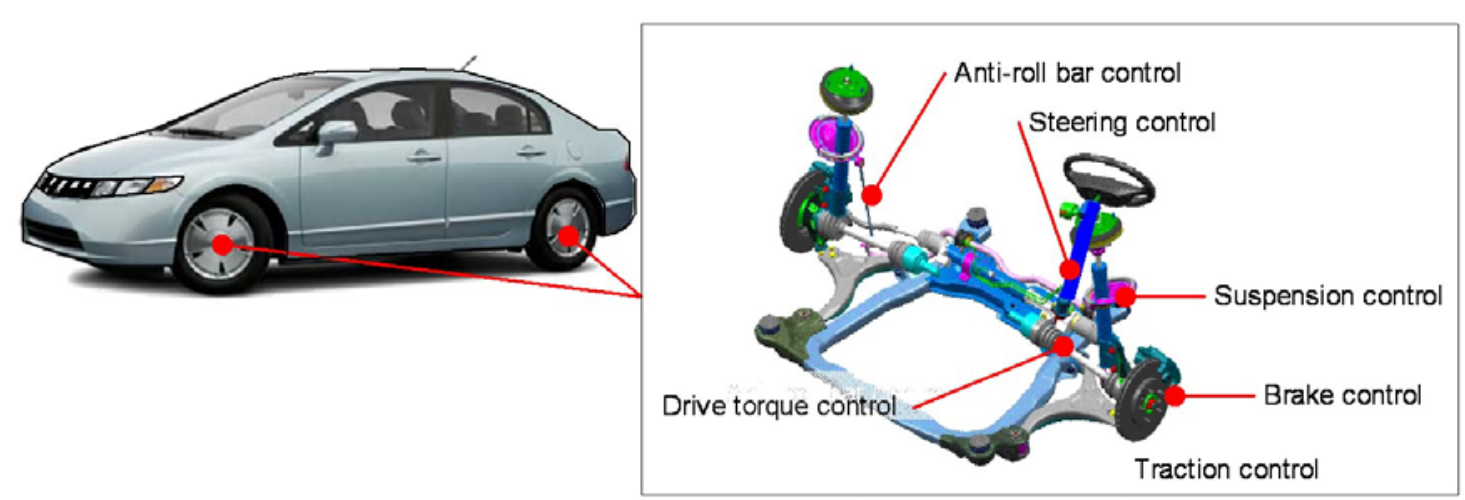

Fig. 2.1 Examples of chassis actuators for vehicle control systems

As chassis control comprises increasing numbers of chassis actuators to achieve multiple control objectives, the amount of software (e.g. control logic) and hardware (e.g. sensors or microcomputer) for each actuator system has increased. Moreover, automotive manufacturers face various combinations of control systems designed in response to diverse customer demands and the different hardware combinations from different suppliers, despite having related functionality. This diverse development environment of vehicle control systems requires that control algorithms provide ways of reducing duplicate hardware or adopting various actuator combinations in order to save resources (e.g. vehicle cost and human workload). Thus, integrated vehicle control applied to active safety systems has been extensively developed. Integrated control aims 
to improve vehicle performance from efficiency viewpoint rather than a simple hardware integration perspective (Ghoneim et al., 2000; Gordon et al., 2003).

\subsubsection{WHAT ARE THE INTENDED BENEFITS OF AN INTEGRATED VEHICLE CONTROL SYSTEM?}

These days integrated vehicle control for handling improvement and active safety has become important because the number of available chassis actuators has increased. Moreover, automotive manufacturers aims to reduce costs by using high performance ECUs (Electronic control unit) and sharing sensors between different sub-actuator systems as well as increasing flexible compatibility for various control options and different hardware suppliers.

The intended benefits of integrated vehicle control systems can be summarized in terms of the improvements in multiple-objectives (Fruechte et al., 1989; Tanaka et al., 1992; Hirano et al., 1993; Ghoneim et al., 2000; Gordon et al., 2003; Koehn et al., 2006):

- improving safety and comfort

- reducing complexity of the control design

- reducing system costs

- providing design flexibility and 'plug-and-play' extensibility in terms of actuator system modularity

- enhancing system reliability

- diagnosing and monitoring system conditions

\subsection{INTEGRATED VEHICLE CONTROL APPLICATIONS}

Integrated vehicle control has been studied intensively and various control techniques have been designed with different chassis actuators: brake control, active steering control, drive torque vectoring, active suspension and other control systems. These different control techniques include model matched-control, which uses a model 
inversion (Horiuchi et al., 1999; Nagai et al., 1999), robust $H_{2}$ and $H_{\infty}$ design methods (You and Chai, 1999; Kitajima and Peng, 2000), and nonlinear predictive control (Tøndel and Johansen, 2003; Borrelli et al., 2005; Falcone et al., 2007a). Other standard techniques are also employed in integrated vehicle control, for example, a direct output feedback methods (Mastinu et al., 1994; Rodic and Vukobratovic, 1999), sliding mode control (Lim and Hedrick, 1999; Mokhiamar and Abe, 2002; Mokhiamar and Abe, 2005), model reference (Wang and Nagai, 1999; Komatsu et al., 2000; Hac and Bodie, 2002), fuzzy logic (Economou and Colyer, 2000; Zeyada et al., 2000; Gordon et al., 2003) and artificial neural Networks (Nwagboso et al., 2002).

The potential benefits of the integrated vehicle control can be interpreted by a ' $\mathrm{g}$ g diagram' which includes vehicle lateral and longitudinal accelerations of vehicle mass center, as shown in Fig. 2.2. The 'g-g diagram' represents the predictable authority of the vehicle controls in terms of lateral and longitudinal accelerations within the fixed limits of vehicle and road surface friction (Tanaka et al., 1992; Gordon et al., 2003).

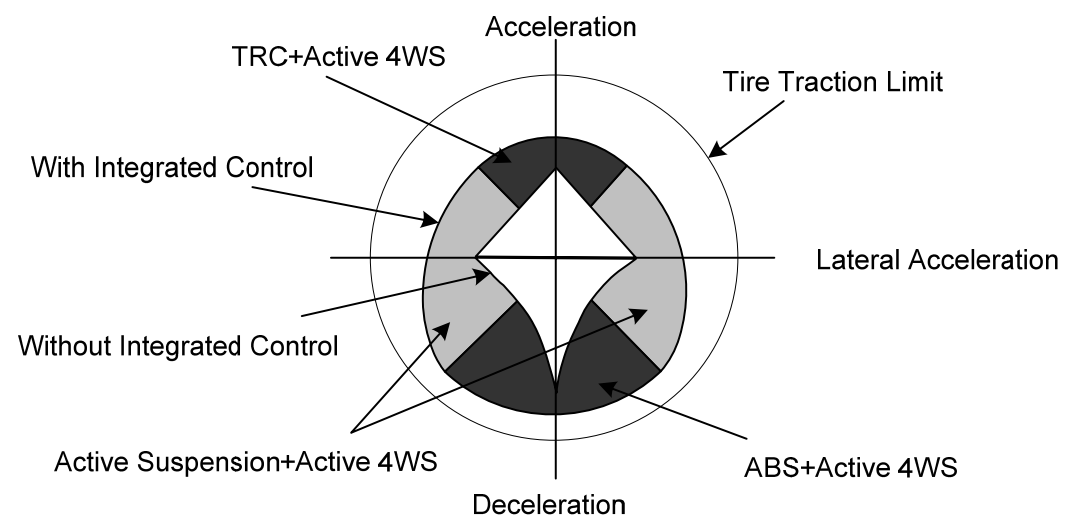

Fig. 2.2 Integrated control and the g-g diagram (Tanaka et al., 1992; Gordon et al., 2003)

Hac and Bodie (2002) analyzed actuator authority in terms of corrective yaw moment generation. In their study, the integrated control rule was based on a simplified 3DOF vehicle model and a nonlinear tire model to calculate the corrective yaw moment 
authority of each subsystem as a control rule. The subsystems consist of active front/rear wheel steer, active brake control, active roll moment distribution between front and rear using controllable suspension. Using the corrective yaw moment authority of each subsystem, supervisory vehicle level controller determines the actuator apportionment to control vehicle motions. A test was performed to evaluate the performance of the integrated control using brake and MR (Magneto-Rheological) suspension control. However, the actuator authority was determined only by considering a stand-alone actuator control.

A review of research on integrated control was presented by Gordon et al. (2003) who attempted to classify the main structural features of integrated vehicle control systems. They also assessed recent developments in control integration methodologies and formulated an enhanced multi-layer architecture based on explicit coordination functionality. A combination of fuzzy sub-system controllers was demonstrated using simulation to develop integrated control behavior at the vehicle level.

\subsection{INTEGRATED VEHICLE CONTROL ARCHITECTURE}

From the overall control architecture view point, Gordon et al. (2003) described five district topologies for integrated vehicle control systems. In their study, 'topology' was broadly defined to include relevant aspects of causality (triggering), ranking (overriding), and dependency as well as the 'geometric' topology of network. A summary of their approach:

- T1. The physical layout of hardware (e.g. sensors, actuators, microcontrollers, communication links and other hardware)

- T2. The causal connection and relative authority of control actions 
- T3. the connection and flow of information - from sensors, state estimators, control output and other monitoring signals (This level is closely connected with fault diagnosis and condition monitoring)

- T4. The structure of the control algorithms and methodologies

- T5. The underlying structure of the control functional design in terms of a modular design

Among these topologies, T2 and T5 are commonly implemented in vehicle control systems, and T5 topology provides more flexibility in terms of functional modular approach with multi-layer architectures. The multi-layer architecture of T5 topology may consist of Layer 0 for hardware (brake, steering, suspension, etc), Layer 1 for basic software ('ABS', 'TCS', 'RWS', etc), Layer 2 for basic behavior ('Yaw stability', 'Roll stability', etc), and other higher layers.

In multi-layer architecture of integrated vehicle control systems, the actuator apportionment plays a key role in the control design, and several advanced strategies of actuator apportionment introduced in (Gordon et al., 2003), for example, pure subsumption, choosing the largest (modulus) activation signal(voting based activation), artificial neural network, and fuzzy rule-based coordination function. In next Section, the actuator apportionment will be discussed in detail.

\subsection{ACTUATOR APPORTIONMENT}

Actuator apportionment is the process of allocating the distribution of braking torques between the four wheels in order to generate a required yaw moment. Because there are more actuators than desired output, one can generate the required yaw moment is many ways, in which case the most effective or efficient method should be selected. Here we compare a simple "actuate one brake only" rule with one that shares the braking effort between two or more wheels. Rule-based actuator can actually be more general 
than this, and includes 'pure subsumption' and voting based activation, while flexible actuator apportionment can be obtained by optimization techniques as well as artificial neural network and fuzzy rule-based coordination functions. In terms of computational burden, rule-based actuator apportionment requires less computer power than does flexible actuator apportionment because the pre-selected rule can be tuned off-line and implemented as a look-up table. Flexible actuator apportionment, on the other hand, works on-line requiring high computational burden. This computational burden can be overcome by developing computer power.

Compared to the rule-based approach, the advantage of the flexible approach is that the combination of the available actuators can reduce the actuator load by appropriately distributing control commands to all available actuators, potentially enhancing the usage of actuator.

In this section, the advantage of the flexible approach compared to the rule-based approach is explained using vehicle simulations for four-wheel individual brake controls. The rule-based approach assumes single-wheel braking, i.e., braking is applied at the front outer wheel of a turn to correct over-steer vehicle motion, and at the rear inner wheel of a turn to correct under-steer vehicle motion. The simulation was performed using an 8 DoF nonlinear vehicle model (see Appendix A) with an initial speed $80 \mathrm{~km} / \mathrm{h}$ and sine maneuver $(0.5 \mathrm{~Hz}, 60 \mathrm{deg}$ peak handwheel steer). In order to focus on the effect of the actuator apportionment, the brake control is idealized by assuming a perfect slip ratio tracking control corresponding to a perfect wheel slip control.

\subsubsection{COMPARISION OF THE ACTUATOR POTENTIAL FOR YAW MOMENT GENERATION}

In order to test the basic mechanism of yaw motion control conceptually in terms of under-steer correction and over-steer correction, the actuator potential for yaw moment generation $\Delta M_{z P T}$ is defined by the magnitude of the gradient of yaw moment versus 
(a) Handwheel steering input

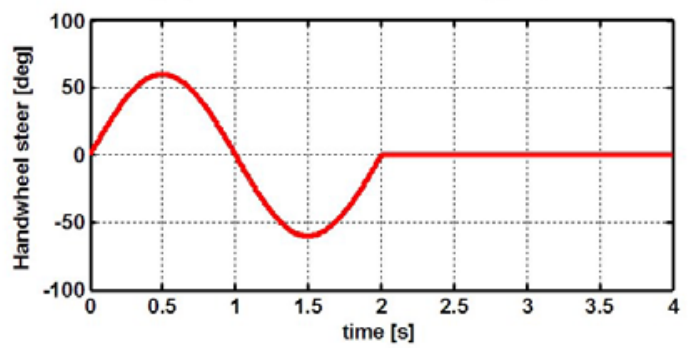

(c) Rule-based apportionment

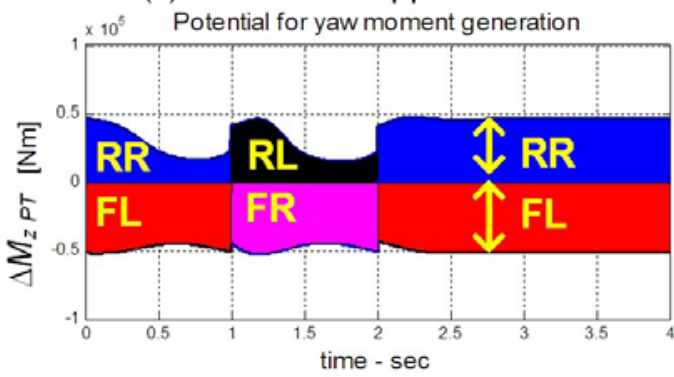

(b) Lateral acceleration response

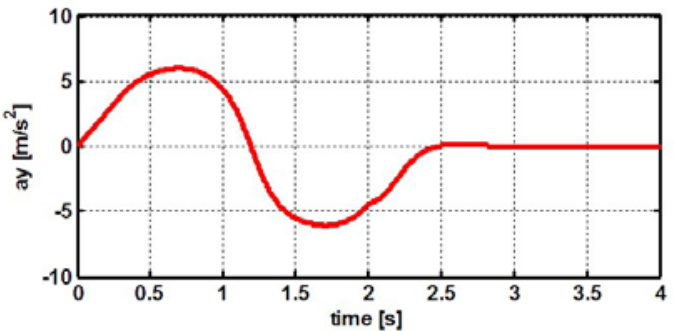

(d) Flexible apportionment

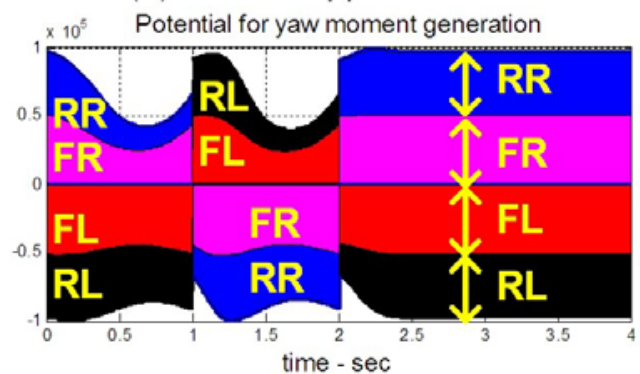

Fig. 2.3 Potential for yaw moment generation using a nonlinear $8 \mathrm{DoF}$ vehicle model (The outer line of (c) and (d) represents the maximum of the potential of yaw moment generation at all wheels)

longitudinal slip ratio from the no-braking condition. The sign of the actuator potential $\Delta M_{z P T}$ is determined to be consistent with the steering direction. The positive potential $\left(\Delta M_{z P T}>0\right)$ corresponds to the action for under-steer correction and the negative potential $\left(\Delta M_{z P T}<0\right)$ represents over-steer correction. In other words, actuator potential for yaw moment generation is positive when the yaw moment gradient is positive for a right turn or negative for a left turn. Moreover, the yaw moment at each wheel is interpreted by the longitudinal and lateral tire forces as well as vehicle geometry

The no braking sine maneuver is given in Fig. 2.3 which shows steering input, lateral acceleration response, and the comparison of the actuator potential for yaw moment generation between the flexible approach using four wheel braking and the rulebased approach using single-wheel braking. In Fig. 2.3 (c) and (d), the different shades of gray denote different wheel positions, and the value of the potential for the yaw moment generation corresponds to the difference between the high and low boundaries 
for each wheel. As shown in Fig. 2.3 (c), the rule-based approach considers only singlewheel braking, e.g. braking at the front outer wheel of a turn for over-steer correction or the rear inner wheel of a turn for under-steer correction. However, the flexible approach in Fig. 2.3 (d) reflects all wheels available to generate desired yaw moment generation. The flexible approach has a bigger potential for yaw moment generation than the rulebased approach because the flexible approach applies braking to all available wheels.

\subsubsection{ACTUATOR LOAD}

In the previous example, no actual braking was applied. Here, the rule-based approach is activated, brakes are applied and simple flexible actuator apportionment is implemented to see the potential for reducing actuator load while maintaining similar kinematic response of the vehicle (equivalent oversteer correction). Fig. 2.4 shows an example case where the flexible approach generates a very similar yaw moment as the rule-based approach. This vehicle simulation was performed with the initial vehicle speed $80 \mathrm{~km} / \mathrm{h}$ and an open-loop straight steering maneuver. In order to compare the brake loads of the rule-based approach and the flexible approach, first, the rule-based control was simulated using a pre-defined sinusoidal slip ratio input. The slip ratio input of the flexible approach was then estimated to track the yaw moment response of the rule-based control with a pre-defined slip ratio. As shown in Fig. 2.4 and Fig. 2.5, while both approaches show similar vehicle responses (yaw moment, yaw rate, sideslip and lateral acceleration), the flexible approach reduces the maximum slip ratio by $58.2 \%$, (0.2 to -0.0835 ) compared with the simple rule-based approach. This is significant because with friction reduced, the flexible approach can deliver the required yaw moment under conditions when the single-wheel rule-based system cannot (the friction limit of the single tire is exceeded). 

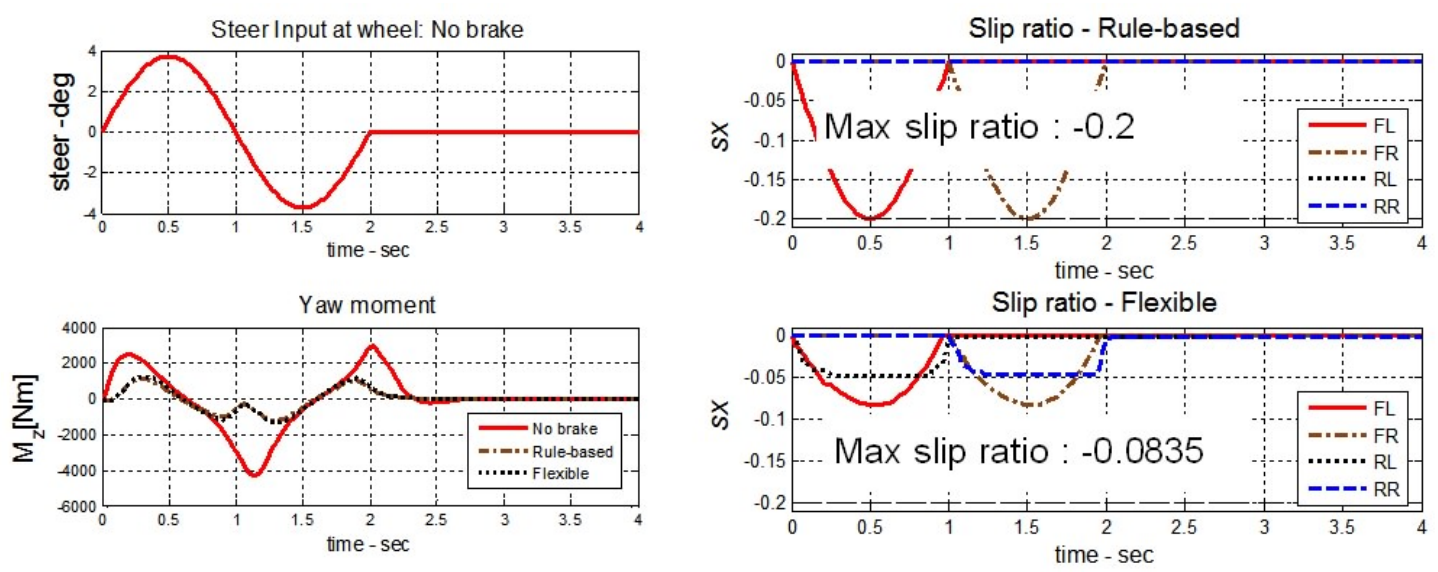

Fig. 2.4 Yaw moment comparison of the rule-based actuator apportionment and the flexible actuator apportionment for sine maneuver.
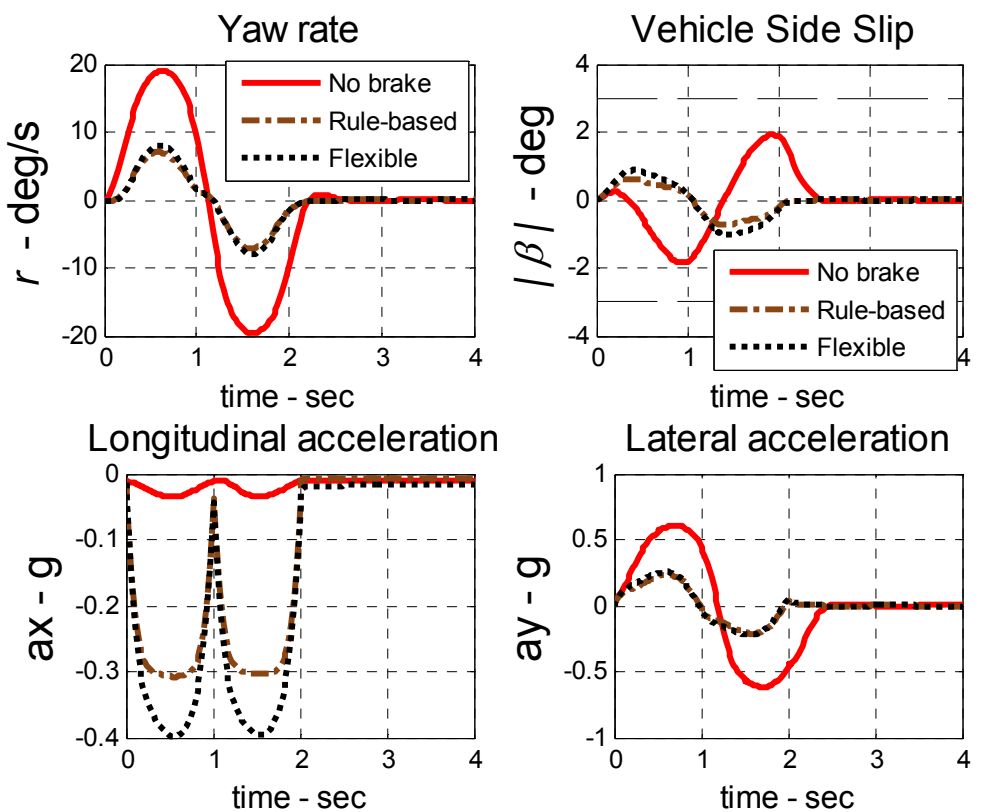

Fig. 2.5 Vehicle responses comparison of the rule-based actuator apportionment and the flexible actuator apportionment for sinusoidal steering maneuver.

The longitudinal acceleration response shown in Fig. 2.5 reveals that the magnitude of the deceleration by the flexible approach is bigger than that resulting from the rule-based approach. This is due to the different tire force distribution of each approach. In the flexible approach, braking to the real wheel reduces the lateral force, while releasing braking force at the front wheel increases the lateral force. The change in 
these lateral forces creates a counter-yaw moment which requires a bigger yaw moment resulting from longitudinal forces. These increased longitudinal forces lead to bigger deceleration than that of the rule-based approach. This bigger deceleration is preferable from the vehicle stability viewpoint because vehicle stability can be achieved by stopping the vehicle. However, if a driver does not want to reduce vehicle speed, the flexible controller may distribute the braking forces as in the rule-based approach at the sacrifice of the actuator load reduction.

The key point to recognize is that flexible actuator apportionment has potential for significantly greater control authority compared to the simple rule-based method, or can achieve the same level of control authority in a more efficient way. As will be developed in this dissertation, the flexible approach also has much greater scope for satisfying multiple objectives, such as combine path and speed tracking, while maintaining overall lateral stability for the vehicle.

\subsection{MODEL PREDICTIVE CONTROL REVIEW}

In this thesis, model predictive control is used to realize flexible actuator apportionment, and this section provides briefly MPC review.

\subsubsection{WHY SHOULD MPC BE USED IN INTEGRATED CHASSIS CONTROL?}

When a system has more than one actuator to realize a control objective, this system is called a redundant actuator system. Examples of these systems include integrated chassis control systems. One of the issues regarding redundant systems is how to apportion redundant actuators. MPC is a possible solution to this actuator apportionment problem, because of its flexibility to handle multi-variables.

MPC is an optimal control methodology that minimizes the tracking error between the predicted future responses of a system and desired responses. This is 
accomplished by considering the minimization of actuators usage as well as the physical limit of the actuator or system response. The tracking error and the actuator efficiency are the bases for a cost function, and the physical limits are implemented as constraint conditions. As shown in industrial application surveys (Qin and Badgwell, 1997; Qin and Badgwell, 2000; Qin and Badgwell, 2003), MPC has been successfully implemented for many industrial control problems such as chemicals, automotive, aerospace and other industry plants. These broad implementations are based on the following main advantages (Maciejowski, 2002):

- MPC can handle multivariable controls such as multiple-input-multiple-output (MIMO) control with an intuitive cost function in the form of the effects of each actuator

- MPC considers physical constraints such as actuator limit or output limit

- MPC's control procedure can be interpreted as the combination of feedback control and feed-forward control: the feedback control is achieved using measured outputs, measured/estimated states or estimated disturbance, and the feed-forward control is done by the prediction of the future plant motions.

In addition to industrial application surveys, a more comprehensive literature review can be found in (Morari and Lee, 1999; Mayne et al., 2000; Allgower et al., 2004) which also examine theoretical and implementation issues.

\subsubsection{PRINCIPLE OF MPC}

In MPC, the future behaviors of the plant are predicted at each control instant. This allows a sequence of control inputs within the prediction horizon to be determined. The general concept of MPC is illustrated in Fig. 2.6. There, outputs $y(\cdot)$, control inputs $u(\cdot)$ and disturbances $w(\cdot)$ are represented up to their horizons and denoted $H_{P}, H_{U}$ and $H_{W}$, respectively. The notation $\left(^{\wedge}\right)$ indicates a predicted value, and $y_{d}(\cdot)$ 


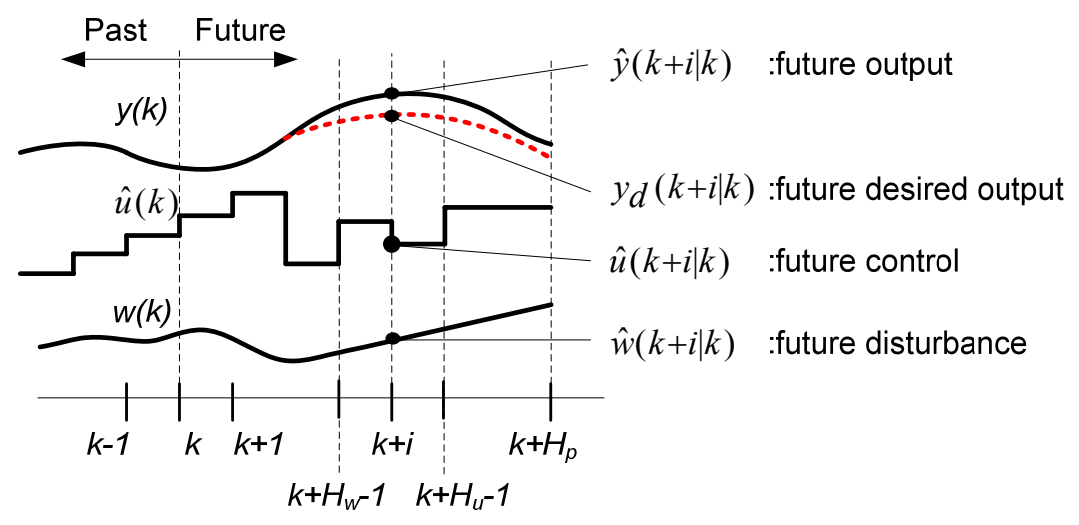

Fig. 2.6 Model Predictive Control concept

corresponds to a desired output. The control sequence $\hat{u}(\cdot)$ is designed to match system performance to the desired motions by minimizing the cost function. Once the full prediction has been obtained, the first value of the control sequence, $\hat{u}(k)$, is used as the control input to the plant, and this process is repeated for each time step.

In mathematical form, the optimization problem is defined to minimize the cost function $J(k)$ at time $t_{k}$ by control moves $\Delta \hat{u}(\cdot)$ as follows:

$$
J(k)_{o p t}=\min _{\Delta \hat{u}\left(t_{k} t_{k}\right), \ldots, \Delta \hat{u}\left(t_{k+H u} \mid t_{k}\right)} J(k)
$$

which is subjected to these constraints

Input constraint:

$$
\begin{aligned}
& \Delta \hat{u}(k+i-1 \mid k) \in \mathcal{U}, \quad i=1, \ldots, H_{u} \\
& \Delta \hat{x}(k+i \mid k) \quad \in \mathcal{X}, \quad i=1, \ldots, H_{P}
\end{aligned}
$$

where constraints $\mathcal{U}$ and $\mathcal{X}$ represent some sets to satisfy the limit of control input and states, respectively. The state constraint in Eq. (2.3) can be substituted by the output constraint depending on the problem. The cost function $J(k)$ at time $t_{k}=T_{s} k$ is the summation of stage cost $L(k+i \mid k)$ up to the prediction horizon $H_{P}$.

$$
\begin{gathered}
\qquad J(k)=\sum_{i=1}^{H_{p}} L(k+i \mid k) \\
L(k+i \mid k)=\|\hat{e}(k+i \mid k)\|_{\mathbf{Q}}^{2}+\|\hat{u}(k+i-1 \mid k)\|_{\mathbf{R}}^{2}+\|\Delta \hat{u}(k+i-1 \mid k)\|_{\mathbf{S}}^{2} \\
\text { where } \hat{e}(k+i \mid k)=\hat{y}(k+i \mid k)-y_{d}(k)
\end{gathered}
$$


Here, $\hat{e}(\cdot)$ is the output tracking error, $\hat{u}(\cdot)$ is the control input, and $\Delta \hat{u}(\cdot)$ is the control move. $\hat{e}(\cdot), \hat{u}(\cdot)$ and $\Delta \hat{u}(\cdot)$ are represented up to their horizons and denoted $H_{P}$ and $H_{u}$ respectively. The stage cost $L(k+i \mid k)$ consists of three terms: output tracking error $\hat{e}(k+i \mid k)$ between predicted output $\hat{y}(k+i \mid k)$ and desired output $y_{d}(k)$, control input $\hat{u}(k+i-1 \mid k)$ and control move $\Delta \hat{u}(k+i-1 \mid k)$. For $k>H_{u}$, the control input and control move are not considered in the stage cost, in other words, $\hat{u}(k+i-1 \mid k)=\Delta \hat{u}(k+i-1 \mid k)=0$ if $k>H_{u}$ in Eq. (2.5). Each of these terms has a weighted norm $\|\cdot\|_{\mathrm{w}}^{2}$ which is defined as $\|\cdot\|_{\mathbf{W}}^{2}:=(\cdot)^{T} \mathbf{W}(\cdot)$ where $\mathbf{W}$ is a diagonal matrix with appropriate dimension.

\subsubsection{APPROACHES TO MPC IMPLEMENTATION}

According to the plant model used to predict the future behaviors of the plant, MPC can be divided into two approaches: linear MPC (LMPC) and nonlinear MPC (NMPC). Table 2.1 shows the general classification of MPC according to plant models.

For LMPC without constraints, the optimal control can be solved by a simple matrix inverse, which makes the derivative of the cost function by control inputs zero, and is similar to conventional proportional control. However, if LMPC is subjected to linear constraints, the optimal control should be calculated using appropriate numerical solvers, such as a quadratic problem solver, because the minimal control of the quadratic cost function may be infeasible due to the constraints.

In NMPC, future plant behaviors can be predicted using a nonlinear plant model directly or an approximated model by the linearization of the nonlinear model. The MPC based on a linearized model (LTV model) can be classified as suboptimal control, while the MPC based on a nonlinear model can be called optimal control. The linearized model of the LTV-MPC can be constructed using direct linearization in terms of Jacobian matrices of the nonlinear dynamics (Prett and García, 1988; Borrelli et al., 2005; Falcone et al., 2007a) or reformulating the nonlinear dynamics into a linear parameter varying 
Table 2.1 General classification of MPC approaches

\begin{tabular}{|l|l|l|}
\hline Approach & Plant model & Description of optimization problem \\
\hline $\begin{array}{l}\text { Linear MPC } \\
\text { (LMPC) }\end{array}$ & LTI model & $\begin{array}{l}\text { i)No constraint: Analytical optimal control } \\
\text { using a matrix inverse } \\
\text { (similar to proportional control) } \\
\text { ii)With linear constraint: optimal control } \\
\text { using appropriate numerical solvers }\end{array}$ \\
\hline $\begin{array}{l}\text { Nonlinear MPC } \\
\text { (NMPC) }\end{array}$ & Nonlinear model & $\begin{array}{l}\text { Optimal control using nonlinear optimization } \\
\text { numerical solvers }\end{array}$ \\
\cline { 2 - 3 } & linear time-varying(LTV) \\
& model (Linearized model) & $\begin{array}{l}\text { Suboptimal control using appropriate } \\
\text { numerical solvers according to linear or } \\
\text { nonlinear constraints. }\end{array}$ \\
\hline
\end{tabular}

(LPV) model (Kothare et al., 2000; Chisci et al., 2003; Keviczky and Balas, 2005) . The LPV scheme depends on the characteristics of the dynamic systems, whereas the direct linearization approach can be applied to any type of dynamic systems. Compared to LMPC, NMPC requires more complicated numerical solvers for optimization problems; the resulting expensive computational cost and reliability of the solver have led to intensive research to find more efficient algorithms in real-time (Qin and Badgwell, 2000; Allgower et al., 2004).

\subsubsection{MODEL FORMULATION}

In MPC, a model should be able to represent a real plant because the performance of MPC is determined by the cost function, which includes the tracking error between the predicted outputs and desired outputs. If the predicted outputs are wrong the good performance of MPC cannot be expected. The choice of a linear or nonlinear model is dependent on plant characteristics. If the operation range of a plant is close to steady state a linear model can well describe the plant behaviors near the steady state operation range. However, if the plant is operated in the transient range and has nonlinear 
behaviors from this different operation range, a nonlinear model should be adopted to predict future plant behaviors.

Model formulation can be achieved by directly using analytical models or using model identification techniques, depending on the plant information. Analytical models can be derived from physical dynamics or energy conservation laws. These analytical models can be represented as state space models or transfer function models for LMPC, and can be used directly as nonlinear functions such as ODE, PDE and DAE for NMPC. However, when plant systems include too many states or complex dynamics, the derivation of analytical models is difficult. As an alternative approach of analytical model representation, model identification techniques can be used; for example, these include input-output models, neural network models / fuzzy models / ARMA (autoregressive moving average) polynomial models, FIR (finite impulse response) models, and other models (Morari and Lee, 1999; Maciejowski, 2002).

For vehicle dynamic systems, there exist relatively accurate analytical nonlinear models as given in Appendix A. Even though vehicle parameters or the nonlinearity of vehicle systems have uncertainties, the accuracy of a vehicle model can be improved by parameter estimation.

\subsubsection{COMPUTATIONAL APPROACHES}

Regarding the optimization solver, various MPC algorithms can be found in the literature (Maciejowski, 2002; Allgower et al., 2004). In LMPC, the quadratic cost function with linear constraint can be solved using standard QP solvers such as active-set optimization or interior-point methods. As one alternative form of the cost function, the quadratic cost function can be transformed into a linear matrix form, which can be solved using linear matrix inequality (LMI) with linear constraints. As another alternative form of the standard quadratic cost function, multiparametric programming considers current 
states as a set of parameters that represents the pieces of the state-space. In this approach, the control gain of each piece is calculated by an off-line computation of the QP problem. Using this off-line computation, the on-line MPC applies the pre-calculated control gain according to the current states. Although this multiparametric programming is efficient for a small number of constraints with fast actuators, due to the increase of the memory size required for the pre-calculated gains it may be infeasible for a system having large numbers of constraints (Maciejowski, 2002).

For NMPC, the numerical solver requires reliability and efficiency for real-time implementation, because the nonlinearity of plants can lead to complexity in the optimal solution. For example, the solution of the NMPC problem can be a global optimum or a local optimum, depending on the numerical algorithm which should have an algorithm to terminate its computation because the solver cannot find a solution within a finite time. The global optimization approach includes genetic algorithms (GA), and the local optimization approach includes Sequential Quadratic Programming (SQP). From the efficiency viewpoint, multiparametric nonlinear programming (mp-NLP) is a real-time implementable nonlinear solver which operates since it combines off-line and on-line approaches based on the approximation of the nonlinear systems with multiparametric programming for linear systems. However, for this mp-NLP the number of states should be carefully chosen because if there are too many parameters they can not be handled appropriately due to computational limits (Tøndel and Johansen, 2005). As an alternative approach to NMPC, the LTV-MPC approach provides a practical means for real-time implementation, even though it requires higher computational burden compared to Linear MPC approaches. This computational burden can be overcome with the evolution of computational power and the convex quadratic optimization approach of the LTV-MPC (Falcone et al., 2007a; Falcone et al., 2007b). 


\subsubsection{MPC STABILITY THEORY}

Because activated constraint conditions cannot be considered by any conventional linear stability approaches such as Root-locus methods or Nyquist criteria, one of the critical issues of MPC theory is how to guarantee the stability of controlled systems,. For this reason, the stability theory of MPC has been studied extensively for the last three decades, and several MPC stability theories have been implemented successfully under certain assumptions (Morari and Lee, 1999; Mayne et al., 2000; Allgower et al., 2004). These assumptions include control feasibility, which means that there exists a control sequence that can steer any initial state to a set point or an equilibrium point. This control feasibility is limited by the control constraint and initial states. For example, when the actuator cannot supply the required control force due to actuator saturation, it is impossible to improve the response except for by maintaining the saturated control force.

In this section, four different MPC stability theories are briefly considered; (i) terminal constraints, (ii) terminal cost and/or terminal constraint based on maximal output admissible sets, (iii) infinite horizon, and (iv) contracting constraint.

\section{Terminal constraints:}

The terminal constraints steer the state to the equilibrium point at the end of the prediction horizon, as given in Eq. (2.6).

$$
x\left(k+H_{p} \mid k\right)=0
$$

Stability can be proven in the sense of Lyapunov by assuming no model error and no disturbance. The Lyapunov function can be constructed using the cost function given in Eq. (2.4). The optimal cost function and the optimal control sequence are respectively denoted as $V^{*}(k)$ and $\pi^{*}(k)=\left[u^{*}(k \mid k), \ldots, u^{*}\left(k+H_{u}-1 \mid k\right)\right]^{T}$ at time $k$. The optimal response at time $k+1$ can then be achieved by applying the optimal control sequence calculated at the previous time step while holding the control input constant after time $k+H_{P}$, as in: 


$$
\pi(k+1)=[\underbrace{u^{*}(k \mid k), \ldots, u^{*}\left(k+H_{u}-1 \mid k\right)}_{N_{u} \times\left(N_{u} \times H_{u}\right)}, \underbrace{u^{*}\left(k+H_{u}-1 \mid k\right)}_{N_{u} \times 1}]^{T}
$$

By the terminal constraints from Eq. (2.6), the terminal stage cost at time $k+1$ is

$$
L^{*}\left(k+1+H_{P} \mid k+1\right)=0
$$

From Eqs. (2.4) and (2.7), the cost function at time $k+1$ can be written in terms of the optimal cost function and the optimal stage cost at time $k$.

$$
\begin{aligned}
V^{*}(k+1) & =\sum_{i=1}^{H_{p}} L^{*}(k+1+i \mid k+1)=\sum_{i=1}^{H_{p}} L^{*}(k+i \mid k)+L^{*}\left(k+1+H_{P} \mid k+1\right)-L^{*}(k+1 \mid k) \\
& =V^{*}(k)-L^{*}(k+1 \mid k)
\end{aligned}
$$

Thus, the monotonic characteristic of the cost function is obtained by rewriting Eq. (2.8) because the stage cost is $L^{*}(k+1 \mid k) \geq 0$ :

$$
V^{*}(k+1)-V^{*}(k)<-L^{*}(k+1 \mid k)
$$

This approach, which is quite simple and intuitive, guarantees the stability by steering the state to the equilibrium point, or the set point at the end of the prediction horizon. However, this terminal constraint can cause the optimal solution to be infeasible because it is an equality constraint which may be difficult to satisfy. Moreover, a large computation load may not be solvable in a finite time for real-time implementation. Thus, infeasibility is the main problem of this approach.

\section{Terminal cost and/or terminal constraint based on maximal output admissible sets:}

To address the infeasibility problem of the terminal equality constraint, various alternative approaches are proposed and implemented by relaxing the terminal constraints given in Eq. (2.6) with the terminal cost and/or terminal constraint given in Eq. (2.10). These alternative approaches are designed to drive the state into a specified terminal constraint set $X_{0}$, which contains the origin, and then switch to some other control law, which can be guaranteed to stabilize the system for initial conditions within $X_{0}$.

$$
\hat{x}(k+N \mid k) \in X_{0} \text { and } L(x, u)=0 \text { if } x \in X_{0}
$$


This method using terminal cost and/or terminal constraint includes various approaches: sub-optimal nonlinear MPC, dual-mode predictive control scheme, sliding mode control based MPC. However, the computation of the admissible set $X_{0}$ is not easy from the practical viewpoints and won't be considered further in this work.

\section{Infinite horizon}

The cost function with an infinite horizon consists of two parts; one is the summation of the stage cost up to a finite control horizon similar to Eq.(2.5), and the other is the cost to stabilize the terminal state at the end of the control horizon up to the infinite horizon. The cost with the infinite horizon can be written mathematically as:

$$
\begin{aligned}
V(k)= & \hat{x}\left(k+H_{u} \mid k\right)^{T} \bar{Q} \hat{x}\left(k+H_{u} \mid k\right)+\sum_{i=1}^{H_{u}}\left\{\|\hat{z}(k+i-1 \mid k)\|_{Q}^{2}\right. \\
& \left.+\|\Delta \hat{u}(k+i-1 \mid k)\|_{R}^{2}+\|\hat{u}(k+i-1 \mid k)\|_{S}^{2}\right\}
\end{aligned}
$$

where $F^{T} \bar{Q} F=\bar{Q}-C_{z}^{T} Q C_{z}=\sum_{i=H_{u}+1}^{\infty}\|\hat{z}(k+i-1 \mid k)\|_{Q}^{2}$

Here, $\hat{z}$ is the output tracking error, $C_{z}$ is the output matrix of a discrete state-space equation, $F$ is an appropriate sequential matrix, and $\bar{Q}$ is the penalty matrix for the infinite horizon, which can be obtained off-line by solving the algebraic Riccati equation for stable linear systems. For unstable linear systems, the plant should (i) be stabilized using state feedback control plus prediction control, or (ii) be decomposed into its stable part and unstable part. For the decomposed plant, the unstable part should be constrained to reach the terminal conditions given in Eqs. (2.6) or (2.10) at the end of control prediction horizon. However, the implementation of this approach is limited for linear time invariant systems, and hence is not applicable to nonlinear vehicle control considered here.

\section{Contracting constraint}

The final approach considered here for MPC stability analysis, a contracting constraint is imposed upon the predicted states to provide the monotonic decrease in their 


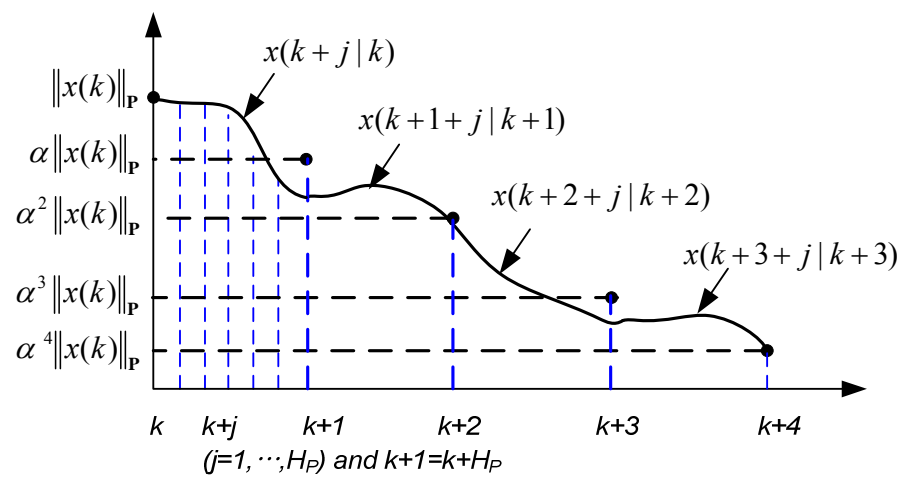

Fig. 2.7 Exponential decay of the state trajectory (de Oliveira Kothare and Morari, 2000)

magnitude along the prediction horizon. The advantage of this approach is that it avoids excessively complicated calculation of the terminal set. The monotonic decrease of states magnitude can be achieved by defining a contracting ratio based on the contracting time interval. This contracting time interval can be a flexible value or a fixed value depending on the optimization formula.

Polak and Yang (Polak and Yang, 1993a; Polak and Yang, 1993b; Yang and Polak, 1993) considered the contracting time interval as a variable with control sequence in the optimization problem. Thus, the nonlinear optimization problem given in Eq. (2.12) determines an optimal control sequence $u$ and an optimal horizon $N^{o}(x)$, which is corresponding to the contracting time interval with contracting constraint. The contracting constraint is defined as a weight norm of the state, and is applied only to the states at the contracting time interval.

$$
P^{c t r}(x): V^{0}(x)=\min _{(u, N)}\left\{V^{*}(x,(\mathbf{u}, N)) \mid \mathbf{u} \in u_{N}(x), N \in\left\{1, \ldots, N_{\max }\right\}\right.
$$

Contracting constraint : $M\left(x\left(k+N_{x}^{0} ; x\right)\right) \leq \alpha M(x(k))$

where $x(k)$ is the plant stateat time $k$ and $M(x)=(1 / 2) x^{T} \mathbf{P} x$

Morari and De Oliveira(1998) proposed a contracting constraint based on two time intervals as shown in Fig. 2.7: One is the sample time interval $(j)$ and the other is 
the contracting time interval $(k)$. The Contracting constraint remains constant during the contracting time interval.

Contracting constraint: $\|x(k+1 \mid k+j)\|_{\mathbf{P}} \leq \alpha\|x(k \mid k)\|_{\mathbf{P}}, \alpha \in[0,1), \mathbf{P}>0$

$$
\text { and } t_{j} \in\left[t_{k}, t_{k+1}\right] \text { or } t_{k+1}=t_{k}+H_{P}, j \in\left[1, \ldots, H_{P}\right]
$$

However, the drawback of this approach is the slow convergence of the contracting condition because the exponential convergence is guaranteed only the time interval, $H_{P}$, which is equal to the prediction horizon. On the other hand, the contracting constraint can allow transient responses during the contacting time interval to be a little larger than the exact monotonic decrease. This means that the contracting constraint can be less strong compared to other stability constraints (e.g., Terminal constraints, Terminal cost, and Infinite horizon) which are sufficient conditions to guarantee stability and in some cases may cause the control to be too severe. This characteristic of the contracting constraint allowing transient motions can be more preferable for vehicle control systems because too severe control inputs can lead to quick tire force saturation. Some issues to implement the contracting constraint to vehicle control systems are discussed in Section 3.3 .

\subsection{SUMMARY}

The general topic of vehicle control architecture has been briefly reviewed based in part on the approach given by Gordon et al., (2003). The trend of the integrated control architecture is to pursue a multi-layer design which consists of integrated layers that specify reference vehicle motions, nonintegrated layers that control lower level actuator systems, and the intermediate layers for actuator apportionment.

Actuator apportionment plays a key role in the control design in terms of actuator modularity and flexibility. Apportionment has been simply classified according to whether it is rule-based or flexible. To demonstrate the benefits of flexible actuator 
apportionment, the apportionment of four wheel individual braking control was investigated by the simulation of an $8 \mathrm{DoF}$ nonlinear vehicle model. The simulation studies showed that the appropriate combination of the braking control at each wheel can reduce the actuator load in terms of smaller applied maximum wheel slip, unlike singlewheel rule-based braking control which does not use the potential for the yaw moment generation at other wheels. The key point to recognize is that flexible actuator apportionment has potential for significantly greater control authority compared to the simple rule-based method. In other words, the flexible approach has much greater scope for satisfying multiple objectives, such as combine path and speed tracking, while maintaining overall lateral stability for the vehicle, as will be developed in this dissertation.

As a solution to realize flexible actuator apportionment, MPC was reviewed briefly with respect to the principle of the MPC, the overview of MPC approaches, model formulation, computational approaches, and MPC stability theory.

The following chapter describes the details of our proposed integrated chassis controller which is based on a model-based hierarchical structure to realize flexible actuator apportionment in a vehicle control system. 


\section{CHAPTER 3}

\section{INTEGRATED CHASSIS CONTROL DESIGN}

The main purpose of the proposed controller is to provide a total vehicle control solution in an active safety system. In other words, the proposed controller aims to satisfy the multiple objective demands of evolutional active safety systems based on integrated chassis control rather than individual stand-alone control, as is the case with $\mathrm{ABS}, \mathrm{ACC}, \mathrm{VSC}$ or other chassis controls. In this thesis, the proposed controller is referred as flexible integrated chassis control (FICC) because chassis control systems are mainly used as actuator systems. The FICC consists of three layers: (1) vehicle motion control as an upper layer, (2) an intermediate layer for the actuator apportionment using Model Predictive Control (MPC) and (3) a lower layer for individual actuator control. For the actuator systems in the lower layer, the brake system and active front steering system are assumed to be high-band width actuators for future X-by-wire actuation which includes as electro-hydraulic brakes (EHB), electro-mechanical brakes (EMB) or electronic power steering (EPS) - see References (Stence, 2004; Huh et al., 2005; Yao, 2006).

In this thesis, the proposed control approach is limited because the decision of control mode switching or timing of driver's input overriding(Seiler et al., 1998; Mai et al., 2000; Hac and Dickinson, 2006; Zhang et al., 2006) will not be covered. Further, road environmental information such as road boundary and obstacle detection (PrakahAsante et al., 2003) is assumed available for simplicity in order to focus on the development of control logic. The road environmental information can be acquired from various devices such as camera, radar, vehicle states measurement devices, vehicle 


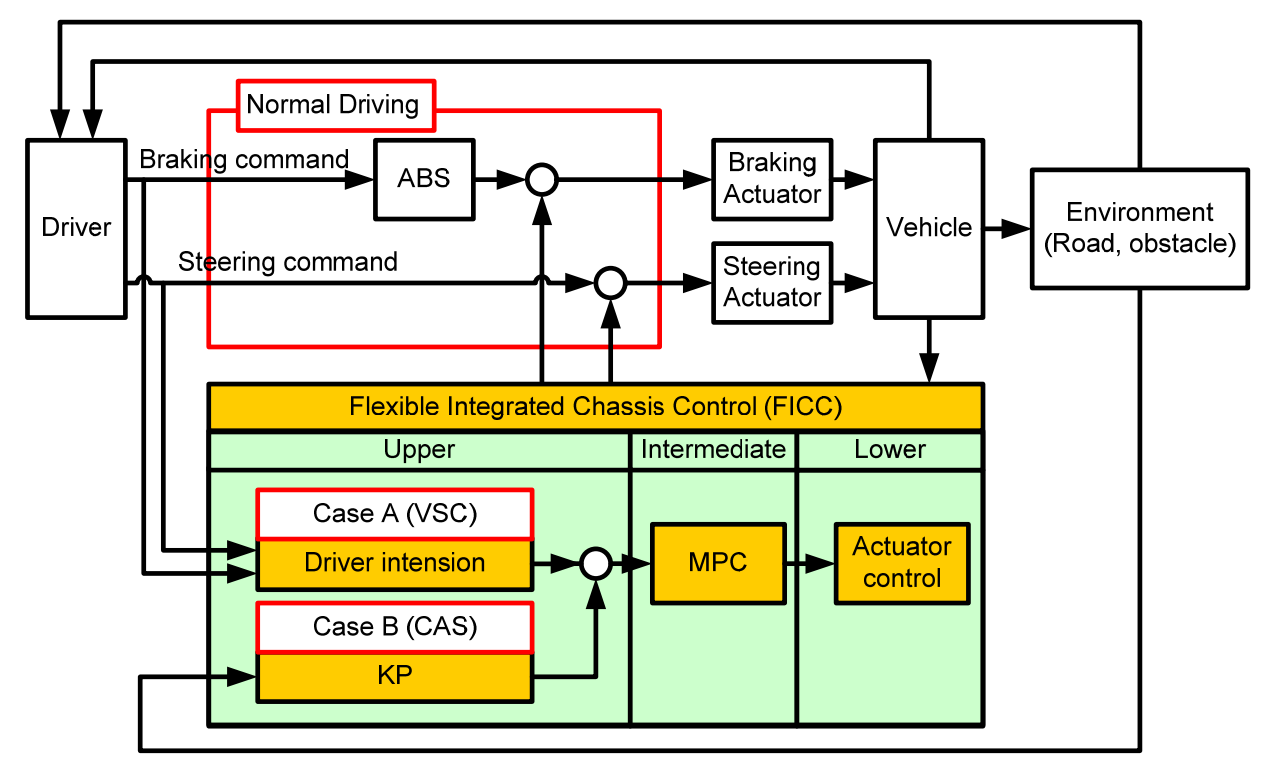

Fig. 3.1 Hierarchical control architecture of flexible integrated chassis control

infrastructure integration (VII), which is designed for communication between vehicles (or with roadside infrastructure) for safety, and other applications.

The overall controller structure is presented in Section 3.1. Section 3.2 provides the vehicle motion control as an upper layer. In Section 3.3, the actuator apportionment in the intermediate layer is described by discussing MPC implementation in vehicle stability control systems. The stand-alone actuator control in a lower layer is presented in Section 3.4. Finally, Section 3.5 summarizes the proposed controller design.

\subsection{OVERALL CONTROLLER STRUCTURE}

In this section, a model-based hierarchical control strategy is presented. The overall FICC consists of the following three control layers:

(a) upper layer: a vehicle motion controller calculates desired vehicle motions by tracking the driver's intention or collision avoidance commands in terms of desired longitudinal speed, sideslip and yaw rate 
(b) intermediate layer: this tracks desired vehicle states by apportioning desired tire forces in appropriate forms of intermediate control commands, for example, reference slip ratios at the four wheels for braking control or steering angle for active front steering control

(c) lower layer: actuator control tracks the desired intermediate control commands

These three layers represent the basic modularity of the controller. When the control objectives are subdivided, a top level control objective can be adopted at the upper layer and any actuator control module can be used at the lower layer. The intermediate layer coordinates the lower layer systematically to achieve the control objectives of the upper layer.

In order to demonstrate the functional adaptation of the FICC in the upper layer, three different driving modes are considered in this study: a normal driving model, a VSC mode (Case A in Fig. 3.1), and a CAS mode (Case B in Fig. 3.1). The normal driving mode corresponds to moderate driving maneuvers, in which the driver's commands (e.g. braking and steering inputs) go directly into the actuator systems. The FICC can override the normal driving mode depending on driver's commands and vehicle states. For example, Case A (Fig. 3.1) of the upper layer represents the VSC control which aims to track driver commands as interpreted from brake pedal input and handwheel steer input, while maintaining vehicle stability simultaneously. On the other hand, Case B (Fig. 3.1) of the upper layer represents an autonomous control mode without direct driver commands. This case represents incorrect driver judgment or unawareness. In addition to the functional adaptation in the upper layer, the inherent flexibility of the control system is provided by the intermediate layer, which employs a form of MPC to determine actuator apportionment for braking and steering control. The MPC uses a linearized model of a nonlinear vehicle model (with 3DoF motion, longitudinal, yaw and side slip) at successive time instants about non-equilibrium operating points to enable on-line optimization. The detailed data flow of the overall FICC control system in terms of 
inputs and outputs of each layer is fully described in Appendix E.3 along with auxiliary modules such as estimation module and other modules.

\subsection{VEHICLE MOTION CONTROL}

The goal of vehicle motion control is to provide reference vehicle motions in terms of longitudinal speed, sideslip and yaw rate. These reference vehicle motions are determined by control modes, for instance, vehicle stability control and collision avoidance control. For the VSC design, FICC provides more longitudinal motion control compared to conventional VSC design consisting of only lateral and yaw motion control. This reference longitudinal motion represents the driver's deceleration intent which can be interpreted from the brake pedal position or brake master cylinder pressure. The reference yaw motion is interpreted from the driver's steering command using a simple steady state bicycle model. The reference sideslip motion aims to provide stability and prevent the vehicle from spinning out. On the other hand, the CAS is designed to provide autonomous maneuvers by supposing that driver commands are not available. Thus the longitudinal speed and yaw rate should be calculated by CAS control logic. More details on the CAS design are described in Chapter 6.

\subsubsection{YAW RATE TRACKING}

Yaw rate tracking significantly provides directional motion control corresponding to a driver's steering command or collision avoidance steering maneuver. For the VSC, the desired yaw rate is defined by the low frequency limit of the transfer function of a bicycle model from (front) steering angle input to yaw rate output:

$$
r_{d}^{*}=G_{r} \delta
$$

where $G_{r}=\frac{r}{\delta}=\frac{V_{x}}{L+K_{u s} V_{x}^{2}}$ and $K_{u s}$ is the target vehicle under-steer gradient. In this study, the reference under-steer gradient has been set to zero; this is for simplicity, and 
clearly other control references could be achieved, e.g. with $K_{u s}>0$. This does not mean that neutral steering is to be achieved, but that sufficiently large deviations from neutral steering should be corrected. On the other hand, the desired yaw rate $r_{d}{ }^{*}$ for the CAS is calculated using Kinematic Policy based on the worst collision threaten which is described in Chapter 6.

The desired yaw rate is limited by a presumed limit on the maximum vehicle cornering force as follows:

$$
r_{d}=\min \left(\left|r_{d}^{*}\right|,\left|\mu_{s} g / V_{x}\right|\right) \cdot \operatorname{sign}(\delta)
$$

This yaw rate tracking control occurs when both the yaw rate error and its percentage exceed their respective thresholds, which aims to prevent the controller from being too sensitive for sensor noise:

$$
\text { If }\left|r-r_{d}\right| \geq r_{\text {threshold }} \text { and }\left|r-r_{d}\right|>\left|r_{d}\right| \cdot r_{\text {threshold_percent }} \text {, activate Yawcontrol }
$$

\subsubsection{VEHICLE SIDESLIP REGULATION}

A reference sideslip motion aims to provide stability and prevent the vehicle from spinning out, where the sideslip is defined by $\beta=\arctan \left(V_{y} / V_{x}\right)$. The reference vehicle sideslip is based on a tolerance band around $\beta=0$, so that the direction of vehicle travel is close to that of the vehicle heading. The following sideslip condition means that the controller is activated only when the current sideslip is greater than the pre-defined threshold of sideslip $\beta_{\text {threshold }}$ :

$$
\text { If }|\beta| \geq \beta_{\text {threshold }} \text { and } \beta \cdot \Delta \beta>0 \text {, activate } \beta \text { control }
$$

where $\Delta \beta=\beta(t)-\beta\left(t-T_{s}\right)$ and $T_{s}$ is the sample time for the vehicle motion controller.

\subsubsection{LONGITUDINAL SPEED TRACKING}

Longitudinal speed tracking controls a vehicle longitudinal motion, and is an additional function of the FICC in contrast conventional VSC based on yaw and sideslip control. 
The longitudinal speed is calculated by a proportional control using a feed-back current acceleration and reference acceleration under the assumption of relatively small lateral velocity.

$$
V_{x \text { ref }}=V_{x}(t)+\left(a_{\text {ref }}+K_{\text {Paccel }}\left(a_{\text {ref }}-a(t)\right)\right) \cdot T_{s}
$$

Here $V_{x \text { ref }}$ is the reference longitudinal speed, and $V_{x}(t)$ is the current longitudinal speed, $a_{r e f}$ is the reference acceleration, and $a(t)$ is the current resultant acceleration of longitudinal and lateral accelerations, $K_{P a c c e l}$ is the constant proportional gain, and $T_{s}$ is the sample time.

The reference acceleration is filtered by a maximum road surface friction, similar to the yaw rate case:

$$
a_{r e f}=\min \left(a_{r e f}, \mu_{s} g\right)
$$

In addition, to prevent the controller from being too sensitive against sensor noise, the controller is activated only when the tracking error of the acceleration is greater than the pre-defined threshold of acceleration tracking error $a_{\text {threshold }}$ :

$$
\text { If }\left|a_{\text {ref }}-a(t)\right| \geq a_{\text {threshold }}, \text { activate } V_{x} \text { control }
$$

\subsection{ACTUATOR APPORTIONMENT}

The intermediate level controller determines the desired tire force vector at each wheel using input reference vehicle motions (longitudinal speed, yaw rate and sideslip), as well as the control activation signals. In order to achieve the desired tire force vector, this controller combines a predictive performance estimate of the vehicle response under the influence of changing intermediate actuator control input using MPC, where the intermediate actuator control input provides a reference to the low level controller.

While this is not the first attempt at implementing MPC in the context of VSC, the approach adopted here offers potential advantages over previous efforts. Carlson and Gerdes (2003) developed an optimal VSC using MPC, based on a very simple vehicle 
model, linearized about an equilibrium point and with imposed constraint conditions. While linearization leads to an explicit control solution, the equilibrium point restriction appears to be unnecessary. Tøndel and Johansen (2003) designed a lateral stabilization system based on nonlinear constrained predictive control, using a three DoF nonlinear vehicle model that incorporates a nonlinear tire; constraints were imposed on slip ratios, yaw rate, side slip and longitudinal velocity. Unfortunately, the solution involved the use of propriety optimization tools, and the relative complexity of the formulation makes real-time implementation seem challenging at the least.

The approach presented here is intended to avoid the complexity of full nonlinear MPC (avoiding a high computational requirement for real-time implementation), and addresses the vehicle nonlinearity by using time-varying linearization at arbitrary (nonequilibrium) operating points. The linearization of the nonlinear vehicle model allows MPC implementation to follow an efficient linear MPC algorithm (Maciejowski, 2002), while taking account of the important tire nonlinear behavior. This approach is similar to recent work, linear time-varying (LTV) MPC, presented by Falcone et al. in (Falcone et al., 2007a), which is implemented for active steering control in autonomous vehicle systems and their controller was evaluated with a test vehicle in real-time environment as well as simulation studies. Recently, Falcone et al. (2007b) also demonstrated an extension of the actuator systems using braking control as well as steering control, and compared the performance of the different DoF predictive vehicle models (e.g., a 2 wheel model and a 4 wheel model) from the practical implementation viewpoint.

For LTV-MPC, the cost function given in Eq. (2.1) can be rewritten in a linear quadratic form which consists of three components: the tracking errors between desired output vector $\mathbf{Y}_{d}$ and predicted output vector $\mathbf{Y}$ up to prediction horizon $H_{P}$, the predicted control input vector $\mathbf{U}$ and also the changes of the predicted controls $\Delta \mathbf{U}$ up to control horizon $H_{U}$. Depending on the weighting parameters $\left(\overline{\mathbf{Q}}_{y}, \overline{\mathbf{Q}}_{u}\right.$ and $\left.\overline{\mathbf{Q}}_{\Delta u}\right)$, the 
cost function minimization seeks to balance tracking performance against control input magnitude and excursions. In mathematical form:

$$
\min _{\Delta \mathbf{U}}\left(J=\frac{1}{2}\left(\mathbf{Y}_{d}-\mathbf{Y}\right)^{T} \overline{\mathbf{Q}}_{y}\left(\mathbf{Y}_{d}-\mathbf{Y}\right)+\frac{1}{2} \mathbf{U}^{T} \overline{\mathbf{Q}}_{u} \mathbf{U}+\frac{1}{2} \Delta \mathbf{U}^{T} \overline{\mathbf{Q}}_{\Delta u} \Delta \mathbf{U}\right)
$$

The desired output vector $\mathbf{Y}_{d}$ comes from the upper vehicle motion control in terms of reference longitudinal speed, sideslip and yaw rate. The predicted output vector $\mathbf{Y}$ is calculated using a predictive vehicle model, which is described in detail in Section 4.2.

Moreover, the cost function $J$ is quadratic and positive definite, and can be solved by a standard quadratic programming for the constraint minimization problem subjected to the following linear matrix inequality constraint:

$$
\mathbf{A}_{\text {const }} \Delta \mathbf{U} \leq \mathbf{b}_{\text {const }}
$$

Here $\mathbf{A}_{\text {const }}$ and $\mathbf{b}_{\text {const }}$ are appropriated matrix forms corresponding to the constraint conditions including actuator physical limits or performance limit. Finally, the first value of the control sequence is used as the control input to the plant, and this process is repeated at each time step. The detailed method is fully presented in Chapter 4 .

Regarding stability constraints, it does not seem to be appropriate to directly implement the existing stability constraints to vehicle control systems for the following reasons:

- The existing stability constraints described in Section 2.5.6 are sufficient conditions to guarantee stability on equilibrium problems or set-point tracking problems, and are aimed to steer the states or Lyapunov function to an equilibrium point by decreasing monotonically.

- However, the vehicle control during severe transient maneuvers is a time varying reference trajectory tracking problem, not a regulation problem. In other words, a particular set-point is not clear because driver's intension can be only interpreted using driver's operations such as steering wheel angle, brake pedal position and accelerator pedal position. 
- Moreover, aggressive control is not always a desired control action for vehicle control systems because of undesirable jerking motion struggling against comfortable ride or tire force saturation leading to steerability loss.

From the practical viewpoint, the strong constraint can be relaxed by allowing transient response boundary to be a little larger than the exact monotonic decrease. Further the solution infeasibility problem resulting from uncertainty can also be addressed by adding a slack variable into the cost function which is similar to a funnel cost approach which minimizes the violation of the constraints and steering the state inside of the contracting boundary toward a set-point. In this approach, a funnel-cost based contracting constraint, may be less restricted against the weights of the cost function because when the slack variable is activated the optimal control tends to minimize the cost due to the constraint violation by overriding the pre-defined weights of the cost function. However, for the rapid time varying reference trajectory tracking problem including VSC systems this funnel-cost based contracting constraint should be more carefully tuned and still have the difficulty to prove its theoretical stability.

\section{$3.4 \quad$ ACTUATOR CONTROL}

In the lower layer, the brake system and active front steering system are assumed to be high-band width actuators for future X-by-wire actuation such as a electro-hydraulic brake (EHB), electro-mechanical brake (EMB) or electronic power steering (EPS) - see References (Stence, 2004; Huh et al., 2005; Yao, 2006). The wheel slip controllers employ Sliding Mode Control (SMC) which is designed based on the general SMC method (Slotine and Li, 1991), and the steering system is simplified as a first-order system.

\subsubsection{WHEEL SLIP CONTROLLER}


The sliding mode controller (SMC) is designed based on the general SMC method - see Slotine and Li (1991). Brake torque $T_{b i}$ is used a control input of the $i$-th wheel, and can be represented by an equivalent control torque $T_{b i, e q}$ plus a switching control torque $T_{b i, s w}, T_{b i}=T_{b i, e q}+T_{b i, s w}$. For simplicity, the brake torque is derived with omission of the subscript $i$. The equivalent control is derived from the sliding surface $\sigma \equiv \dot{\tilde{x}}+\gamma \tilde{x}=0$, where $\tilde{x}=s_{x}-s_{x d}$ and $s_{x d}$ denotes a desired slip ratio and $\gamma$ is a positive constant. The switching control is designed to drive the slip ratio to the sliding surface $(\sigma=0)$ and is defined as $T_{b, s w}=-K \operatorname{sat}(\sigma)$, where $K$ is a switching control gain and $\operatorname{sat}(\sigma) \equiv \min \{1,|\sigma|\} \cdot \operatorname{sgn}(\sigma)$ is the saturation function.

Following the standard SMC design process, the control torque input can be obtained in the following form, with guaranteed stability:

$$
T_{b}=\hat{F}_{x w} R_{w}+\left(1+s_{x}\right) \hat{\dot{u}}_{x w} \frac{I_{w}}{R_{w}}-\gamma\left(s_{x}-s_{x d}\right) \frac{u_{x w} I_{w}}{R_{w}}-K \operatorname{sat}\left(\frac{\sigma}{\Phi}\right)
$$

where

$$
K=D_{1}+I_{w} \frac{\left(1+s_{x}\right)}{R_{w}{ }^{2}} D_{2}+\gamma \frac{u_{x w} I_{w}}{R_{w}{ }^{2}}\left|s_{x}-s_{x d}\right|, \quad D_{1}=d_{1}\left|\hat{F}_{x w}\right| \geq\left|\hat{F}_{x w}-F_{x w}\right|, \quad D_{2}=d_{2}\left|\hat{\dot{u}}_{x w}\right| \geq\left|\hat{\dot{u}}_{x w}-\dot{u}_{x w}\right|
$$

and $\gamma, \Phi, d_{1}$ and $d_{2}$ are design parameters. The switching control gain $K$ is obtained analytically by using the stability condition of a Lyapunov function. The candidate Lyapunov function is defined as $V=\tilde{x}^{2} / 2$, and uses an estimated longitudinal tire force $\hat{F}_{x w}$ and a longitudinal acceleration $\hat{\dot{u}}_{x w}$ of each wheel center. This represents a general form of the controller design, with error bounds on state and force estimation. The approximate longitudinal tire force and acceleration are to be bounded within $D_{1}$ and $D_{2}$, respectively, and are proportional to the maximum error percentage $\left(d_{1}, d_{2}\right)$ of their estimation values. In addition, to avoid the chattering problem due to the imperfect switching control under physical limits of the actuator or model uncertainty, the saturation function is used with the boundary layer thickness $\Phi$ around the sliding surface. A more detailed derivation of the wheel slip controller is presented in Appendix 
B. The investigation of the effects of different sliding surface designs on the wheel slip controller can be found in (Shim et al., 2007).

\subsubsection{ACTIVE FRONT STEERING CONTROLLER}

The steering system is simplified as a first-order system. In other words, the intermediate steering control provided by the MPC is imported into the vehicle model through a first order transfer function with time constant $\tau_{s}=0.05 \mathrm{~s}$ and steering ratio $1 / 16$. More complicated steer-by-wire model and control approaches can be found in $\mathrm{Cu}$ et al., 1998; Amberkar et al., 2004; Verschuren and Duringhof, 2004; Park et al., 2005)

\subsection{SUMMARY}

Flexible integrated chassis control (FICC) is proposed as a means to provide a total vehicle control solution in an active safety system, particularly in terms of two control modes: vehicle stability control and collision avoidance control. The FICC consists of three layers: (1) vehicle motion control as an upper layer, (2) an intermediate layer for the actuator apportionment using Model Predictive Control (MPC) and (3) a lower layer for individual actuator control. In this chapter, the upper layer and intermediate layer design were described in detail as the main control logic of our proposed controller. The upper layer determines reference vehicle motions corresponding to vehicle stability control or collision avoidance control in terms of longitudinal speed, yaw rate, and side slip. In the intermediate layer, the MPC design is based on the linear time-varying MPC in order to avoid the complexity of full nonlinear MPC and to address the vehicle nonlinearity by using time-varying linearization at arbitrary (non-equilibrium) operating points. In the lower layer, the brake system and active front steering system are assumed to be high-band width actuators for future X-by- 
wire actuation. For the braking control, SMC is used for wheel slip ratio tracking by providing a fast switch control around sliding surface.

The following chapter provides more detailed MPC design, and several strategies for VSC system implementation are presented with respect to predictive vehicle models in MPC. 


\section{CHAPTER 4}

\section{MODEL PREDICTIVE CONTROL}

This chapter provides more detailed linear time-varying (LTV) MPC design which plays a key role of the actuator apportionment of the FICC. Several strategies for MPC predictive model were presented according to the vehicle model freedom of degree and various chassis actuators. Moreover, MPC design parameter tuning is discussed with respect to the weights of the cost function and actuator constrains.

The basic idea of the LTV-MPC can be found in several early process control studies (Prett and García, 1988; Maciejowski, 2002), and it has been recently implemented to chassis control systems for path following (Falcone et al., 2007a; Falcone et al., 2007b). However, MPC problem formulation depends on target systems, for example, states, outputs, disturbance, cost function, and the matrix form of predictive output sequence. Thus the proposed MPC design is formulized to be suitable for vehicle control systems.

The proposed MPC design is described in detail in Section 4.1. Then Section 4.2 discusses the implementation of MPC on vehicle control systems. Finally, Section 4.3 summarizes findings and presents overall conclusions from the study.

\subsection{LINEAR TIME-VARYING MPC DESIGN}

The proposed MPC is designed based on LTV-MPC in order to avoid the complexity of full nonlinear MPC and to address the vehicle nonlinearity by using timevarying linearization at arbitrary (non-equilibrium) operating points. In this section, the 
MPC design starts from the derivation of the difference predictive model equation for the prediction output sequence, and a linear quadratic cost function will be formulated as an optimization problem.

\subsubsection{DIFFERENCE EQUATIONS AT NON-EQUILIBRIUM POINTS}

Consider the following continuous nonlinear dynamical systems $\dot{x}(t)$ and output variables $y(t)$.

$$
\begin{gathered}
\dot{x}(t)=f(x(t), u(t), w(t)) \\
y(t)=g(x(t))
\end{gathered}
$$

Here $x(t) \in R^{N x}$ is the vector of states at time $\mathrm{t}, f: R^{N x} \rightarrow R^{N x}$ is the nonlinear vectorvalued function, $u(t) \in R^{N u}$ is the input control input, $w(t) \in R^{N w}$ is the input disturbance vector, $y(t) \in R^{N y}$ is the output vector and $g: R^{N y} \rightarrow R^{N y}$ is the nonlinear vector-valued function. The initial operating points $\left(x_{0}, u_{0}, w_{0}\right)$ are not required to be equilibria. At $t_{0}$ the following differential equation and initial conditions are presumed known:

$$
\dot{x}_{0}=f\left(x_{0}, u_{0}, w_{0}\right), x_{0}=x\left(t_{0}\right), u_{0}=u\left(t_{0}\right), w_{0}=w\left(t_{0}\right)
$$

The linearized differential equation at a point $(x, u, w)$ located near the initial point $\left(x_{0}, u_{0}, w_{0}\right)$ can be approximated by the first two terms in a Taylor series expansion:

$$
\begin{gathered}
\dot{x} \approx f\left(x_{0}, u_{0}, w_{0}\right)+\mathbf{A}_{c}\left(x-x_{0}\right)+\mathbf{B}_{c}\left(u-u_{0}\right)+\mathbf{E}_{c}\left(w-w_{0}\right) \\
y_{k}=\mathbf{C} x_{k}
\end{gathered}
$$

where

$$
\mathbf{A}_{c}=\frac{\partial}{\partial x} f\left(x_{0}, u_{0}, w_{0}\right), \mathbf{B}_{c}=\frac{\partial}{\partial u} f\left(x_{0}, u_{0}, w_{0}\right), \mathbf{C}=\frac{\partial}{\partial x} g\left(x_{0}\right) \text { and } \mathbf{E}_{c}=\frac{\partial}{\partial w} f\left(x_{0}, u_{0}, w_{0}\right)
$$

are the Jacobian matrices. The difference equation in terms of time step $k$ can be derived from the continuous Eq. (4.4) using the Euler approximation for the time derivative:

$$
\dot{x}_{k} \approx \frac{x_{k+1}-x_{k}}{T_{s}}=\frac{\Delta x_{k+1}}{T_{s}}=f\left(x_{0}, u_{0}, w_{0}\right)+\mathbf{A}_{c}\left(x_{k}-x_{0}\right)+\mathbf{B}_{c}\left(u_{k}-u_{0}\right)+\mathbf{E}_{c}\left(w_{k}-w_{0}\right)
$$

where $\Delta x_{k+1}=x_{k+1}-x_{k}$. Rearranging this equation: 


$$
\Delta x_{k+1}=T_{s} f\left(x_{0}, u_{0}, w_{0}\right)+T_{s} \mathbf{A}_{c}\left(x_{k}-x_{0}\right)+T_{s} \mathbf{B}_{c}\left(u_{k}-u_{0}\right)+T_{s} \mathbf{E}_{c}\left(w_{k}-w_{0}\right)
$$

In order to express the above difference equation in a more useful form, the constant term $f\left(x_{0}, u_{0}, w_{0}\right)$ is to be eliminated. Using the same notation, consider a second (time-shifted) difference equation, at time $t_{k-1}$ :

$$
\Delta x_{k}=T_{s} f\left(x_{0}, u_{0}, w_{0}\right)+T_{s} \mathbf{A}_{c}\left(x_{k-1}-x_{0}\right)+T_{s} \mathbf{B}_{c}\left(u_{k-1}-u_{0}\right)+T_{s} \mathbf{E}_{c}\left(w_{k-1}-w_{0}\right)
$$

Subtracting Eqs. (4.7) and (4.8) we obtain the linear form

$$
\Delta x_{k+1}=\mathbf{A} \Delta x_{k}+\mathbf{B} \Delta u_{k}+\mathbf{E} \Delta w_{k}
$$

where

$$
\begin{aligned}
& \mathbf{A}=\left(1+T_{s} \mathbf{A}_{c}\right), \mathbf{B}=T_{s} \mathbf{B}_{c}, \mathbf{E}=T_{s} \mathbf{E}_{c} \\
& \Delta x_{k}=x_{k}-x_{k-1}, \Delta u_{k}=u_{k}-u_{k-1}, \Delta w_{k}=w_{k}-w_{k-1}
\end{aligned}
$$

The initial conditions for the difference Eq. (4.9) are then given by

$$
\Delta x_{0}=x_{0}-x_{-1}, \Delta u_{0}=u_{0}-u_{-1}, \Delta w_{0}=w_{0}-w_{-1},
$$

Variables $x_{-1}, u_{-1}, w_{-1}, x_{0}, u_{0}, w_{0}$ are then all to be prescribed at time $t_{0}$.

\subsubsection{PREDICTIVE CONTROL DESIGN}

In MPC, at each control instant, the future behaviors of the plant are predicted to determine a desired sequence of control inputs within the prediction horizon. This sequence is designed to match system performance to the desired motions by minimizing a cost function. Once the full prediction has been obtained, the first value of the control sequence, $u\left(t_{0}\right)$ is used as the control input to the plant, and this process is repeated at each time step. The states, control inputs and disturbances are represented up to their horizons, denoted $H_{p}, H_{u}$ and $H_{w}$, respectively. From the output Eq. (4.5) and the notation $\left(^{\wedge}\right)$ indicating a predicted value at time $t_{0}+k$, the predicted output in the discrete form can be expressed as follows with:

$$
\hat{y}_{k}=\mathbf{C} \hat{x}_{k}
$$

From the Eq. (4.9), the sequence of state vectors is predicted as follows 


$$
\begin{gathered}
\hat{x}_{1}=x_{0}+\mathbf{A} \Delta x_{0}+\mathbf{B} \Delta u_{0}+\mathbf{E} \Delta w_{0} \text { and } \Delta \hat{x}_{1}=\mathbf{A} \Delta x_{0}+\mathbf{B} \Delta u_{0}+\mathbf{E} \Delta w_{0} \\
\hat{x}_{2}=\hat{x}_{1}+\mathbf{A} \Delta \hat{x}_{1}+\mathbf{B} \Delta \hat{u}_{1}+\mathbf{E} \Delta \hat{w}_{1} \\
=x_{0}+\left(\mathbf{A}+\mathbf{A}^{2}\right) \Delta x_{0}+(\mathbf{B}+\mathbf{A B}) \Delta u_{0}+\mathbf{B} \Delta \hat{u}_{1}+(\mathbf{E}+\mathbf{A E}) \Delta w_{0}+\mathbf{E} \Delta \hat{w}_{1} \\
\ldots \\
\hat{x}_{H_{p}}=x_{0}+\sum_{i=1}^{i=H_{P}} \mathbf{A}^{i} \Delta x_{0}+\sum_{i=0}^{i=H_{p}-1} \mathbf{A}^{i} \mathbf{B} \cdot \Delta u_{0}+\sum_{i=0}^{i=H_{p}-2} \mathbf{A}^{i} \mathbf{B} \Delta \hat{u}_{1}+\ldots+\sum_{i=0}^{i=H_{P}-H_{U}} \mathbf{A}^{i} \mathbf{B} \Delta \hat{u}_{H_{U}-1} \\
+\sum_{i=0}^{i=H_{p}-1} \mathbf{A}^{i} \mathbf{E} \cdot \Delta w_{0}+\sum_{i=0}^{i=H_{p}-2} \mathbf{A}^{i} \mathbf{E} \Delta \hat{w}_{1}+\ldots+\sum_{i=0}^{i=H_{p}-H_{W}} \mathbf{A}^{i} \Delta \hat{w}_{H_{W}-1}
\end{gathered}
$$

where $H_{P}>\left(H_{U}-1\right)$ and $H_{P}>\left(H_{W}-1\right)$

The above may be reformulated in compact matrix form, in terms of a predictive output vector $Y$, defined as the difference between predicted outputs (up to the prediction horizon) and the initial output:

$$
\mathbf{Y}=\mathbf{G} \Delta x_{0}+\mathbf{F}_{1} \Delta \mathbf{U}+\mathbf{F}_{2} \Delta \mathbf{W}
$$

where

$$
\begin{aligned}
& \mathbf{Y}=\left[\begin{array}{l}
\hat{y}_{1}-y_{0} \\
\hat{y}_{2}-y_{0} \\
\cdots \\
\hat{y}_{H_{P}}-y_{0}
\end{array}\right], \Delta \mathbf{U}=\left[\begin{array}{l}
u_{0}-u_{-1} \\
\hat{u}_{1}-u_{0} \\
\cdots \\
\hat{u}_{H_{U}-1}-\hat{u}_{H_{U}-2}
\end{array}\right], \Delta \mathbf{W}=\left[\begin{array}{l}
w_{0}-w_{-1} \\
\hat{w}_{1}-w_{0} \\
\cdots \\
\hat{w}_{H_{W}-1}-\hat{w}_{H_{W}-2}
\end{array}\right], \mathbf{G}=\left[\begin{array}{l}
\mathbf{C A} \\
\mathbf{C A}+\mathbf{C A}^{2} \\
\cdots \\
\sum_{i=1} \mathbf{C A}^{i}
\end{array}\right] \\
& \mathbf{F}_{1}=\left[\begin{array}{llll}
\mathbf{C B} & \mathbf{0} & \ldots & \mathbf{0} \\
\mathbf{C B}+\mathbf{C A B} & \mathbf{C B} & \ldots & \mathbf{0} \\
\ldots & & & \\
\sum_{i=0}^{H_{P}-1} \mathbf{C} \mathbf{A}^{i} \mathbf{B} & \sum_{i=0}^{H_{p}-2} \mathbf{C A}^{i} \mathbf{B} & \ldots & \sum_{i=0}^{H_{p}-H_{U}} \mathbf{C A}^{i} \mathbf{B}
\end{array}\right], \mathbf{F}_{2}=\left[\begin{array}{llll}
\mathbf{C E} & \mathbf{0} & \ldots & \mathbf{0} \\
\mathbf{C E}+\mathbf{C A E} & \mathbf{C E} & \ldots & \mathbf{0} \\
\ldots & & \\
\sum_{i=0}^{H_{P}-1} \mathbf{C} \mathbf{A}^{i} \mathbf{E} & \sum_{i=0}^{H_{P}-2} \mathbf{C} \mathbf{A}^{i} \mathbf{E} & \ldots & \sum_{i=0}^{H_{p}-H_{W}} \mathbf{C A}^{i} \mathbf{E}
\end{array}\right]
\end{aligned}
$$

As described in Section 3.3, the cost function is defined with three components: the errors between desired outputs $y_{d}(t)$ and predicted outputs $\hat{y}(t)$, the predicted control inputs and also the changes of the predicted controls. Depending on the weighting 
parameters, the cost function minimization seeks to balance tracking performance against control input excursions. In mathematical form:

$$
\begin{aligned}
J & =\frac{1}{2} \sum_{j=1}^{H_{P}}\left[y_{d}\left(t_{0}+j\right)-\hat{y}\left(t_{0}+j\right)\right]^{T} \mathbf{Q}_{y}(j)\left[y_{d}\left(t_{0}+j\right)-\hat{y}\left(t_{0}+j\right)\right] \\
& +\frac{1}{2} \sum_{j=1}^{H_{U}} \hat{u}_{j-1}{ }^{T} \mathbf{Q}_{u}(j) \hat{u}_{j-1}+\frac{1}{2} \sum_{j=1}^{H_{U}} \Delta \hat{u}_{j-1}{ }^{T} \mathbf{Q}_{\Delta u}(j) \Delta \hat{u}_{j-1}
\end{aligned}
$$

or using a more compact notation

$$
J=\frac{1}{2}\left(\mathbf{Y}_{d}-\mathbf{Y}\right)^{T} \overline{\mathbf{Q}}_{y}\left(\mathbf{Y}_{d}-\mathbf{Y}\right)+\frac{1}{2} \mathbf{U}^{T} \overline{\mathbf{Q}}_{u} \mathbf{U}+\frac{1}{2} \Delta \mathbf{U}^{T} \overline{\mathbf{Q}}_{\Delta u} \Delta \mathbf{U}
$$

where

$$
\begin{aligned}
& \overline{\mathbf{Q}}_{y}=\operatorname{diag}\left\{\mathbf{Q}_{y}(1), \ldots ., \mathbf{Q}_{y}\left(H_{P}\right)\right\} \geq 0 \quad \text { and } \mathbf{Q}_{y}(i) \in R^{N y \times N y} \quad i=1,,, H_{P} \\
& \overline{\mathbf{Q}}_{u}=\operatorname{diag}\left\{\mathbf{Q}_{u}(1), \ldots ., \mathbf{Q}_{u}\left(H_{U}\right)\right\} \geq 0 \quad \text { and } \mathbf{Q}_{u}(i) \in R^{N u \times N u} \quad i=1,,,, H_{U} \\
& \overline{\mathbf{Q}}_{\Delta u}=\operatorname{diag}\left\{\mathbf{Q}_{\Delta u}(1), \ldots, \mathbf{Q}_{\Delta u}\left(H_{U}\right)\right\} \geq 0 \text { and } \mathbf{Q}_{\Delta u}(i) \in R^{N u \times N u} \quad i=1,,,, H_{U} \\
& \mathbf{Y}_{d}=\left[y_{d 1}-y_{0}, y_{d 2}-y_{0}, \ldots, y_{d p_{1}}-y_{0}\right]^{T}
\end{aligned}
$$

The control input vector can be rewritten in terms of the change of control inputs as follows:

$$
\mathbf{U}=\left[u_{0}, \hat{u}_{1}, \ldots, \hat{u}_{H_{U}-1}\right]^{T}=\left[u_{-1}+\Delta u_{0}, \ldots, u_{-1}+\sum_{i=0}^{H_{U}} \Delta u_{i-1}\right]^{T}=\mathbf{U}_{-1}+\mathbf{L}_{w} \Delta \mathbf{U}
$$

where $\mathbf{U}_{-1}=\left[I_{N u}, I_{N u}, \ldots, I_{N u}\right]^{T} u_{-1}, I_{N u}$ is an $N u \times N u$ identity matrix and

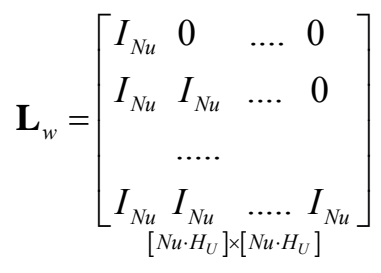

By substituting the predictive output Eq. (4.14) and the control input vector Eq. (4.18) into the cost function Eq. (4.16), the following form is obtained:

$$
\begin{aligned}
J & =\frac{1}{2}\left(\mathbf{Y}_{d}-\mathbf{G} \Delta x_{0}-\mathbf{F}_{1} \Delta \mathbf{U}-\mathbf{F}_{2} \Delta \mathbf{W}\right)^{T} \overline{\mathbf{Q}}_{y}\left(\mathbf{Y}_{d}-\mathbf{G} \Delta x_{0}-\mathbf{F}_{1} \Delta \mathbf{U}-\mathbf{F}_{2} \Delta \mathbf{W}\right) \\
& +\frac{1}{2}\left(\mathbf{U}_{-1}+\mathbf{L}_{w} \Delta \mathbf{U}\right)^{T} \overline{\mathbf{Q}}_{u}\left(\mathbf{U}_{-1}+\mathbf{L}_{w} \Delta \mathbf{U}\right)+\frac{1}{2} \Delta \mathbf{U}^{T} \overline{\mathbf{Q}}_{\Delta u} \Delta \mathbf{U}
\end{aligned}
$$


This cost function (4.19) can be reduced to the standard quadratic form by eliminating the irrelevant constant term:

$$
\min _{\Delta \mathbf{U}}\left(J=\frac{1}{2} \Delta \mathbf{U}^{T} \mathbf{H} \Delta \mathbf{U}+\mathbf{f} \Delta \mathbf{U}\right)
$$

where

$$
\begin{aligned}
& \mathbf{H}=\overline{\mathbf{Q}}_{\Delta u}+\mathbf{F}_{1}^{T} \overline{\mathbf{Q}}_{y} \mathbf{F}_{1}+\mathbf{L}_{w}{ }^{T} \overline{\mathbf{Q}}_{u} \mathbf{L}_{w} \\
& \mathbf{f}=-\left(\mathbf{Y}_{d}-\mathbf{G} \Delta x_{0}-\mathbf{F}_{2} \Delta \mathbf{W}\right)^{T} \overline{\mathbf{Q}}_{y} \mathbf{F}_{1}+\mathbf{U}_{-1}{ }^{T} \overline{\mathbf{Q}}_{u} \mathbf{L}_{w}
\end{aligned}
$$

Since $J$ is quadratic and positive definite, in the absence of constraints, the optimization is simply implemented via the equation $\partial J / \partial \Delta \mathbf{U}=0$. Then the control input at time $t_{0}$ is

$$
\Delta \mathbf{U}=\mathbf{H}^{-1}(-\mathbf{f})
$$

The proposed MPC approach can be extended to include control constraints. The problem becomes a standard quadratic programming form for the constraint optimization problem:

$$
\begin{aligned}
& \min _{\Delta \mathbf{U}}\left(J=\frac{1}{2} \Delta \mathbf{U}^{T} \mathbf{H} \Delta \mathbf{U}+\mathbf{f} \Delta \mathbf{U}\right) \\
& \text { subject to } \mathbf{A}_{\text {const }} \Delta \mathbf{U} \leq \mathbf{b}_{\text {const }}
\end{aligned}
$$

Here $\mathbf{A}_{\text {const }}$ and $\mathbf{b}_{\text {const }}$ are appropriated matrix forms corresponding to the constraint conditions such as actuator physical limits or performance limit. Even though the optimal solution without constraint can be calculated by simple matrix inverse, the weight of the cost function should be tuned carefully to prevent actuator saturation or infeasible actuator command. On the other hand, the optimal approach with constraint provides lots of flexibility to handle actuator saturation limit or failure at the sacrifice of the computational burden increase.

Finally the control input $u_{0}$ at time $k$ is determined by (4.23) where $\Delta \mathbf{U}$ is the solution of the quadratic optimization problem without constraint given in (4.21) or the quadratic optimization problem with constraints given in (4.22):

$$
u_{0}=u_{-1}+\Delta u_{0}
$$


where $\Delta u_{0}=\mathbf{I}_{m} \Delta \mathbf{U}$ and $\mathbf{I}_{m}=\left[I_{N u}, \operatorname{zeros}\left(N u, N u \cdot H_{U}\right)\right]$

\subsection{IMPLEMENTATION OF MPC ON VEHICLE CONTROL SYSTEMS}

The actuator apportionment on chassis control systems is achieved by the MPC with a predictive vehicle model, which estimates a predictive performance of the vehicle response under the influence of changing actuator control input. This section describes several different predictive vehicle models and the MPC tuning parameters.

\subsubsection{DEGREE OF FREEDOM OF VEHICLE MODEL}

For a predictive model, more accurate vehicle response is fundamentally expected with a high order degree of freedom model. However, due to computational burden or hardware limitations, a simple model is preferred from a practical implementation viewpoint. For example, a 2 DoF nonlinear vehicle model (lateral and yaw dynamics) can be used for a conventional VSC design based on yaw and sideslip control and a 3 DoF nonlinear vehicle model (longitudinal, lateral and yaw dynamics) can be used for more longitudinal control compared to the conventional VSC design. Moreover, the predictive vehicle model can be expanded flexible depending on control purpose, i.e., roll dynamics for roll-over prevention.

The predictive model can be constructed by the following procedure. First, consider a 3 DoF nonlinear vehicle model with four wheels where the vehicle dynamics equations in terms of longitudinal speed $V_{x}$, lateral speed $V_{y}$ and yaw rate $r$ can be written as

$$
\begin{gathered}
\dot{V}_{x}=\frac{1}{m}\left[r V_{y}+\sum_{i=1}^{4} F_{x i}\right] \\
\dot{V}_{y}=\frac{1}{m}\left[-V_{x} r+\sum_{i=1}^{4} F_{y i}\right] \\
\dot{r}=\frac{1}{I_{z z}}\left[c_{f}\left(F_{x 1}-F_{x 2}\right)+c_{r}\left(F_{x 3}-F_{x 4}\right)+a\left(F_{y 1}+F_{y 2}\right)-b\left(F_{y 3}+F_{y 4}\right)\right]
\end{gathered}
$$


Table 4.1 State space components of a predictive vehicle model

\begin{tabular}{|c|c|c|c|}
\hline & $2 \mathrm{DoF}$ & $3 \mathrm{DoF}$ & Remark \\
\hline State & $\begin{array}{c}x=\left[V_{y}, r\right]^{T} \text { or } \\
x=[\beta, r]^{T}\end{array}$ & $x=\left[V_{x}, V_{y}, r\right]^{T}$ & $\begin{array}{l}\text { In a } 2 \text { DoF vehicle model, a } \\
\text { longitudinal speed is assumed as } \\
\text { constant. }\end{array}$ \\
\hline Output & $y=[\beta, r]^{T}$ & $y=\left[V_{x}, \beta, r\right]^{T}$ & Desired vehicle motions \\
\hline $\begin{array}{l}\text { Intermediate } \\
\text { actuator input }\end{array}$ & $\begin{aligned} u & =\left[\vec{s}_{x}\right]^{T} \text { or } \\
u & =\left[\delta_{f}\right] \text { or } \\
u & =\left[\vec{s}_{x}, \delta_{f}\right]^{T}\end{aligned}$ & $\begin{aligned} u & =\left[\vec{s}_{x}\right]^{T} \text { or } \\
u & =\left[\delta_{f}\right] \text { or } \\
u & =\left[\vec{s}_{x}, \delta_{f}\right]^{T}\end{aligned}$ & $\begin{array}{l}\text { Depending on the combination } \\
\text { of available actuators }\end{array}$ \\
\hline Disturbance & $\begin{array}{c}\text { No } w \text { model or } \\
w=\delta_{f}\left(\text { if } \delta_{f} \not \subset u\right)\end{array}$ & $\begin{array}{c}\text { No } w \text { model or } \\
w=\delta_{f}\left(\text { if } \delta_{f} \not \subset u\right)\end{array}$ & $\begin{array}{l}\text { Depending on a disturbance } \\
\text { model }\end{array}$ \\
\hline
\end{tabular}

Here $m$ is a vehicle sprung mass, $I_{z z}$ is the yaw moment of inertia w.r.t. $z$-axis, $a$ and $b$ are the distance of c.g to front and rear axles, and $c_{f}$ and $c_{r}$ are the half track of front and rear axles, respectively. $F_{x i}$ and $F_{y i}$ are tire forces in vehicle $x$ and $y$ directions at $i$-th tire contact patch. The nonlinear tire force generation is represented by the combined slip tire model based on Pacejka's Magic formula, a similarity model, as functions of tire longitudinal slip ratio, tire lateral slip ratio, a vertical load and surface friction coefficient (Pacejka, 2002; Milliken and Milliken, 2003; Gordon and Best, 2006).

The linearized first-order state-space equation at non-equilibrium points can be written as follows in terms of Jacobian matrices, which are calculated numerically by considering small perturbations of each variable:

$$
\dot{x}=f\left(x_{0}, u_{0}, w_{0}\right)+\mathbf{A}_{c}\left(x-x_{0}\right)+\mathbf{B}_{c}\left(u-u_{0}\right)+\mathbf{E}_{c}\left(w-w_{0}\right)
$$

Each state, output, input and disturbance can be established depending on control strategies as shown in Table 4.1.

Here, slip ratios $\vec{s}_{x}=\left[s_{x 1}, s_{x 2}, s_{x 3}, s_{x 4}\right]^{T}$ and front steering input $\delta_{f}$ correspond to the individual four wheel braking control and active front steering control, respectively. The outputs mean performance variables. For a disturbance model, the bandwidth of the 
steering input is assumed sufficiently small that a constant steering rate can be used, i.e. the disturbance model takes the form $\dot{\delta}=$ constant. However, when the controller uses the steering input as an actuator, then the disturbance terms is ignored. The Jacobian matrices for the linearized vehicle model are calculated numerically by considering small perturbations of each variable, based on a nonlinear tire model.

The most flexible actuator apportionment can be realized by considering the largest DoF vehicle predictive model with all available actuators. Then this predictive model can cover all possible MPC predictive models by combining the modification of the weight of cost function and the constraints of actuator control. For example, if reference motions focus on the $2 \mathrm{DoF}$ vehicle motions of yaw and sideslip, then the longitudinal term of the cost function can be ignored by no penalty on the longitudinal weight. Regarding different actuator options, any actuator can be easily decoupled from control systems by constraint its operation range as the zero change of control input. This flexible control structure can provide a solution to adopt various demands of customers and different hardware suppliers without major change of control logic.

\subsubsection{WEIGHTING PARAMETERS IN THE COST FUNCTION}

For simplicity, the weighting parameters in the cost function (4.17) is reduced to a series of constant diagonal matrices as follows

$$
\begin{gathered}
\mathbf{Q}_{y}(i)=\mathbf{Q}_{y}=\operatorname{diag}\left(Q_{y 1}, . ., Q_{y N y}\right) \in R^{N y \times N y}, Q_{y j} \in R \quad\left(i=1,,, H_{P} \text { and } j=1, . ., N y\right) \\
\mathbf{Q}_{u}(i)=\mathbf{Q}_{u}=\operatorname{diag}\left(Q_{u 1}, . ., Q_{u N u}\right) \in R^{N u \times N u}, Q_{u j} \in R \quad\left(i=1, . ., H_{U} \text { and } j=1, . ., N u\right) \\
\mathbf{Q}_{\Delta u}(i)=\mathbf{Q}_{\Delta u}=\operatorname{diag}\left(Q_{\Delta u 1}, . ., Q_{\Delta u N u}\right) \in R^{N u \times N u}, Q_{\Delta u j} \in R \quad\left(i=1, . ., H_{U} \text { and } j=1, . ., N u\right)
\end{gathered}
$$

Here the weight for actuator control change was set to equal that of actuator control magnitude, $Q_{\Delta u}=Q_{u}$. The value of output tracking weight $Q_{y j}$ in Eq. (4.28) can be assigned zero penalty by setting $Q_{y j} \rightarrow 0$ when the activation signal of the output $y_{i}$ tracking control from the upper layer is turned off. For example, if no driver's 
longitudinal demand is specified, we can set $Q_{V_{x}} \rightarrow 0$. Regarding vehicle stability, the vehicle sideslip regulation is designed to have higher priority than yaw rate tracking; thus during any excursion which leads to excessive side-slipping of the vehicle, the priority is to orient the vehicle to the motion path, rather than respond directly to the drivers steering input, as would be the case with prioritizing the yaw rate tracking control. Of course other possibilities exist, and while the present choice appears effective, no claim is made for its optimality. The chosen prioritization is achieved via the following rule:

$$
\text { If ( } \beta \text { control is activated) then } Q_{r} \rightarrow 0 \text {, else } Q_{\beta} \rightarrow 0
$$

\subsubsection{PREDICTION HORIZON AND SAMPLE TIME OF MPC}

Regarding the prediction horizons $H_{P}, H_{U}$ and $H_{W}$, there is a trade-off between the computational load implied by a large prediction horizon, and available improvements in control performance. Based on an earlier study (Chang and Gordon, 2007a), we chose $H_{P}=10, H_{U}=1$ and $H_{W}=10$; here $H_{U}=1$ means that the chosen control is assumed constant throughout the state prediction interval. The most likely reason for this is that the control model does not include a delay in the buildup of tire forces, nor in actuator actuation - for larger values of $H_{U}$, the control sequence increases its bandwidth, while the actual plant model reduces its effect through low-pass filtering. Moreover, the disturbance horizon $H_{W}$ is only considered when the controller does not use steering input as an actuator. We should mention that the larger number of the prediction horizon does not mean better vehicle motion prediction because this LTVMPC is based on the linearization at operating points, in other words, when the system nonlinearity is severe then the linearized model can not predict as accurate as in linear systems. However, the prediction horizon should be able to capture appropriately the change of vehicle motions resulting from the activation of each actuator. 
The sample time of MPC $T_{s}$ need not be the same as that of the measurement and can be considered as a design parameter because it is related to the choice of prediction horizon. The prediction horizon itself is to be large enough to be capable of capturing the control effects on future vehicle motion. Thus, for the any give prediction horizon (in seconds) a smaller sample time implies the need for more data in the control sequence and leads to an increased computational burden. While it is difficult to define optimal values for the sample time, the value $T_{s}=0.02$ was chosen here as "reasonable".

The accuracy of the LTV-model for prediction horizon is investigated by comparing the predicted response to the response of the nonlinear model at an arbitrary time instant. The nonlinear model consists of $8 \mathrm{DoF}$ vehicle dynamics with the nonlinear time model (see Appendix A). The responses of the LTV-model are obtained using the linearization of the $3 \mathrm{DoF}$ nonlinear vehicle model at time $1.4 \mathrm{~s}$ and Eq. (4.29) to predict the future vehicle motions during the prediction interval $T_{\text {prediction }}=0.2 \mathrm{~s}\left(T_{\text {prediction }}=T_{S} \cdot H_{P}\right.$, $H_{P}=10$ and $\left.T_{s}=0.02 s\right)$.

$$
\mathbf{Y}_{\text {prediction }}=\mathbf{G} \Delta x_{0}+\mathbf{F}_{1} \Delta \mathbf{U}+\mathbf{F}_{2} \Delta \mathbf{W}+y_{0}
$$

Here $\mathbf{Y}_{\text {prediction }}$ is the predicted output sequence, and $y_{0}$ represents the output at time $1.4 \mathrm{~s}$. $\mathbf{G}, \mathbf{F}_{1}$, and $\mathbf{F}_{2}$ are given in Eqs. (4.14) .

Fig. 4.1 shows the comparison of yaw and sideslip responses between the nonlinear model and the LTV-model from time $1.4 \mathrm{~s}$ to time $1.6 \mathrm{~s}$ during a sine with dwell test without braking $\left(U_{0}=80 \mathrm{~km} / \mathrm{h}\right.$ and $\left.\mu_{H}=0.9\right)$. The response of the LTV-model shows small deviation from those of the nonlinear vehicle model at the end of the prediction interval, i.e., yaw rate error (5.9\%) and sideslip error (1.8\%). These deviations obviously become larger as the prediction interval increases. 

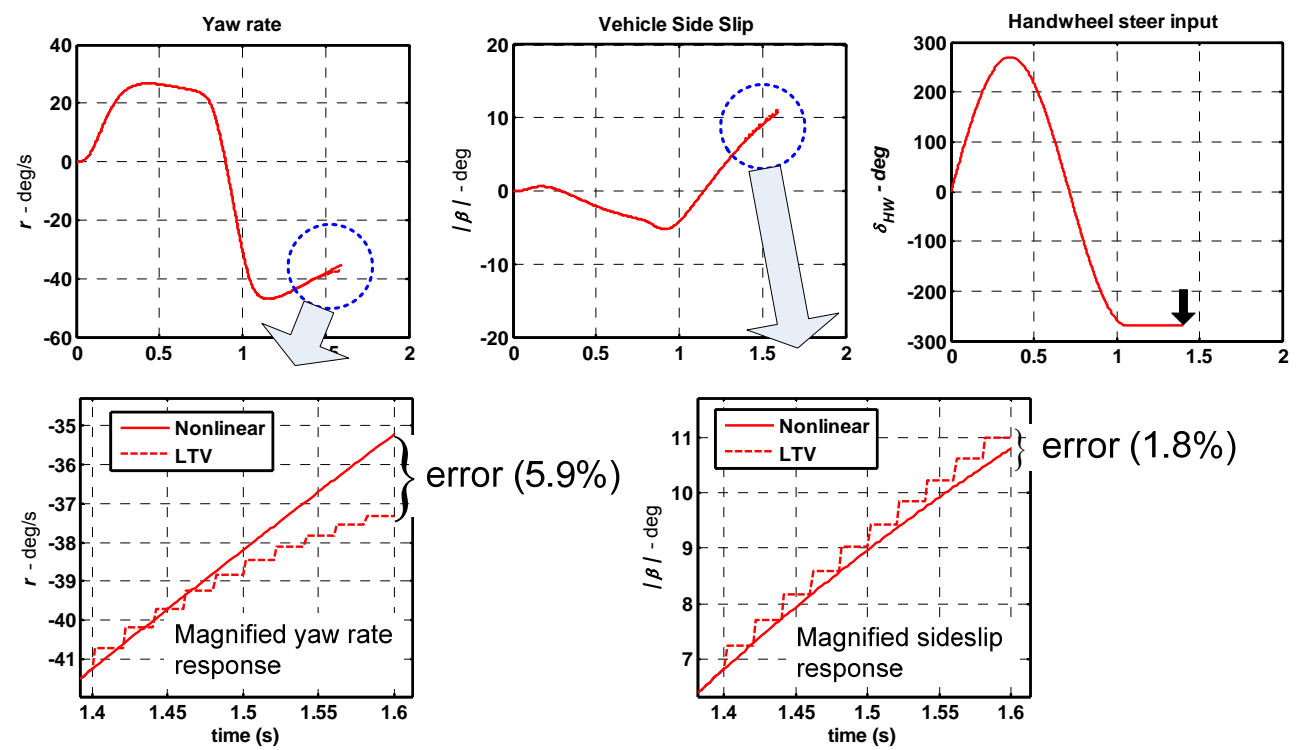

Fig. 4.1 Comparison of a nonlinear model and a LTV-model at instant $1.4 \mathrm{~s}$ during a sine with dwell test (8DoF nonlinear vehicle model and no braking)

\subsubsection{ACTUATOR CONSTRAINTS}

The braking control constraint is intended to avoid excessive actuator demands (to protect system hardware from failure) and also to prevent wheel lock $\left(s_{x}=-1\right)$; this was translated into the form $-0.2 \leq s_{x}+\Delta s_{x} \leq 0$ in order to prevent the large slip ratio from causing a severe loss of steering controllability. Moreover, the steering control has a wheel steer angle limit, 30 degrees, and a wheel steering angle rate limit, $1.636 \mathrm{rad} / \mathrm{s}(=1500 \mathrm{deg} / \mathrm{s}$ measured at the steering wheel).

\subsection{SUMMARY}

For actuator apportionment in the FICC, the proposed MPC is based on linear time-varying (LTV) MPC in order to avoid the complexity of full nonlinear MPC and to address the vehicle nonlinearity by using time-varying linearization at arbitrary (nonequilibrium) operating points. The vehicle predictive model can be constructed using a simple nonlinear vehicle dynamics including control performance states representing 
longitudinal, lateral and yaw motions and available actuator systems. The most flexible actuator apportionment can be realized by considering the largest DoF vehicle predictive model with all available actuators. Then this predictive model can cover all possible MPC predictive models by combining the modification of the weight of the cost function and the constraint of actuator control. Thus, this flexible control structure can provide a solution to adopt various demands of customers and different hardware suppliers without major change of control logic.

Regarding MPC design parameters, there is a trade-off between the computational burden implied by a large prediction horizon, and the available improvements in control performance. For example, the prediction horizon should be able to capture appropriately the change of vehicle motions resulting from the activation of each actuator.

In the following chapter, we will investigate the performance benefits of optimal actuator apportionment based on the proposed MPC approach in vehicle stability control through simulation studies for various driving maneuvers. 


\section{CHAPTER 5}

\section{PERFORMANCE BENEFITS OF OPTIMAL ACTUATOR APPORTIONMENT}

This chapter investigates the performance benefits of vehicle stability control (VSC) using the proposed optimal actuator apportionment, where the purpose of VSC is to achieve desired motion tracking performance (handling responsiveness) while maintaining vehicle stability (handling stability). The vehicle stability control is designed first based on the general framework for flexible integrated chassis control (FICC) described in Chapter 3 using four wheel individual braking control, brake-based VSC. The chassis control systems of the controller is then expanded by adding active front steering control (AFS) to address the actuator failure to control braking. The braking control is commonly used for VSC because ABS can be reused without major mechanical change and yaw moment generation using braking control is effective overall operation ranges including limit driving situations, e.g. severe transient maneuvers or ice road (Koehn et al., 2006; Hac et al., 2007). AFS can directly influence on handling responsiveness during normal driving, however, its capability to control handling responsiveness becomes small during severe maneuvers due to the limit of lateral tire forces (Hac et al., 2007).

In order to investigate the robustness of the controller, the exact model-plant match of MPC is relaxed by introducing model-plant mismatch, i.e., various road surface friction, vehicle state and tire force estimation, and road surface friction estimation. In this study, an Extended Kalman Filter (EKF) is used to estimate vehicle states and tire forces. Although the estimation performance using EKF is quite dependent on system model accuracy as well as sensor accuracy, this drawback can be addressed using vehicle 
parameter identification (Kiencke and Nielsen, 2005) or integrating inertial sensors and GPS (Global Positioning System) with compensating sensor noise and bias (Bevly, 2004). Moreover, the roughly designed EKF used in this study seems to be suitably simple and effective for the relative comparison of the exact known state case. Similar to the vehicle states and tire force estimation, a road surface friction estimation is designed based on a simple logic that uses longitudinal and lateral accelerations by following the design concept presented in (Eckert, 1998), even though several sophisticated road surface friction estimation approaches such as (Liu and Peng, 1996; Gustafsson, 1997; Ray, 1997; Canudas-de-Wit and Horowitz, 1999) are available.

The performance evaluation procedure is described in detail in Section 5.1. Section 5.2 then discusses the performance of the brake-based VSC by comparing different control scenarios. Proposed controller robustness issue is covered in Section 5.3 and addressed model-plant mismatch, actuator time constants, vehicle state estimation, and road surface friction estimation. The performance of more complex maneuvers is presented in Section 5.4 and the actuator failure mitigation is given in Section 5.5. The feasibility of real-timed implementation is discussed in Section 5.6. Section 5.7 finally summarizes findings and presents overall conclusions from the study.

\subsection{BRAKE-BASED VSC IMPLEMENTATION}

\subsubsection{TEST MANEUVER}

Vehicle stability control can be defined in terms of handling stability and handling responsiveness (Hac et al., 2007). Handling stability represents stable vehicle motions by interpreting vehicle sideslip against spin-out or roll angle against roll-over. The handling responsiveness, on the other hand, refers to the sensitivity to the driver's inputs which can be interpreted using the driver's steering input, yaw rate and lateral acceleration response. 


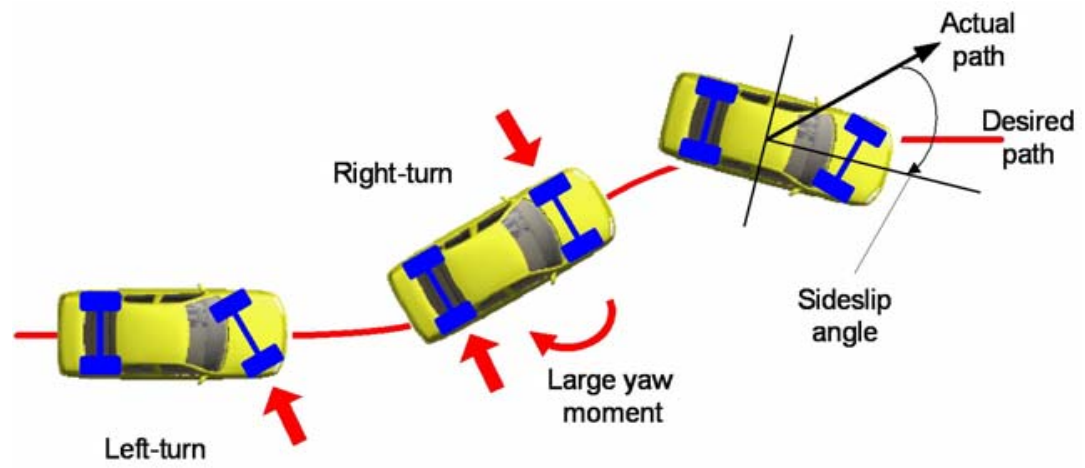

Fig. 5.1 Over-steer vehicle response during emergency lane change maneuver on high friction road - Arrow represents a conceptual lateral tire force at a front axle (Hac and Bodie, 2002)

To evaluate vehicle handling characteristics, several different tests are chosen. An overview of test maneuvers is presented by Hac et al. (2007). Hac et al highlights such tests as (1) steady-state test maneuver (constant radius test), (2) transient test maneuver (single or double lane change), and (3) combined steady-state and transient test maneuvers (step steer or J-turn, and Brake-in-turn). Recently, a 0.7Hz Sine-With-Dwell maneuver proposed by NHTSA has been included among the transient test maneuvers as an open-loop steering maneuver. This Sine-With-Dwell maneuver is intended to provide a repeatable objective test to demonstrate vehicle response qualities of different VSC equipped vehicles, as compared to non-VSC vehicles (NHTSA, 2007). In addition to these pre-defined tests, the worst-case vehicle evaluation methodology is used to define the test maneuver by optimization based on comprehensive and rich test maneuvers. This was done through a simulation study in which all possible steer maneuvers are considered to identify the worst roll-over performance case (Ma and Peng, 1999; Kou et al., 2007) .

Among the above test maneuvers, the Sine-With-Dwell maneuver causes a vehicle to be over-steer due to severe transient maneuvers along with abrupt steering direction change as shown in Fig. 5.1. This over-steer vehicle behavior is useful to identify the benefits of the VSC equipped vehicles compared to no VSC equipped vehicles. 


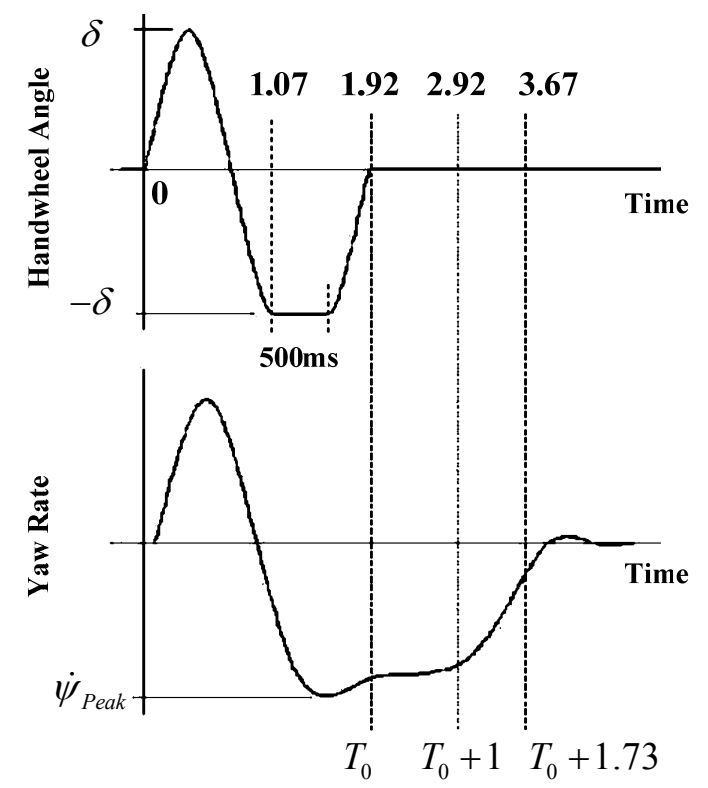

Fig. 5.2 Sine-With-Dwell maneuver steering wheel position and yaw velocity information (NHTSA, 2007)

To identify the benefits of VSC equipped vehicles, the simulation studies are mainly performed with a $0.7 \mathrm{~Hz}$ Sine-With-Dwell maneuver described in Fig. 5.2.

In the evaluation of the controller performance, vehicle motions satisfy the following lateral stability and responsiveness criteria proposed by NHTSA, as given in (5.1) and (5.2):

Lateral stability criteria:

$$
\left\{\begin{array}{l}
\frac{\dot{\psi}_{\left(T_{0}+1.00\right)}}{\dot{\psi}_{\text {Peak }}} \times 100 \leq 35 \% \quad(\text { Criterion } \# 1), \text { and } \\
\frac{\dot{\psi}_{\left(T_{0}+1.75\right)}}{\dot{\psi}_{\text {Peak }}} \times 100 \leq 20 \% \quad(\text { Criterion } \# 2)
\end{array}\right.
$$

Responsiveness Criterion:

$$
\begin{aligned}
& \text { Lateral Displacement }(Y) \text { at } \mathrm{t}=1.07 \\
& \left\{\begin{array}{l}
\geq 1.83 \mathrm{~m}, \text { when } \mathrm{GVWR} \geq 1587.6 \mathrm{~kg}(3,500 \mathrm{lb}) \\
\geq 1.22 \mathrm{~m}, \text { when GVWR }<1587.6 \mathrm{~kg}(3,500 \mathrm{lb})
\end{array}\right.
\end{aligned}
$$

Here $T_{0}$ is the time to the completion of the steering input, and $\dot{\psi}_{\text {Peak }}$ is the first local peak yaw rate generated by the $0.7 \mathrm{~Hz}$ Sine-With-Dwell maneuver. The value $T_{0}=1.92$ was estimated from (NHTSA, 2007). 
In order to satisfy these criteria, the maximum allowable peak handwheel steer $\left|\delta_{H W}\right|_{\text {peak }}$ is first investigated with a handwheel steer limit of 270 degrees. If necessary, a reduced peak handwheel steering angle is used to satisfy the stability criteria compared to the handwheel steer limit. Vehicle responses are then evaluated in terms of the RMS (root mean square) yaw rate error $\left|e_{r}\right|_{R M S}$ representing yaw rate tracking, peak sideslip $|\beta|_{\text {peak }}$ and control effort (for applied braking torque). The yaw rate error $e_{r}$ is defined as the difference between the yaw rate deviation from the desired yaw rate and a threshold of yaw rate deviation given in equations (3.2) and (3.3). This yaw rate error is only considered when the yaw rate deviation exceeds the threshold of yaw rate deviation, while the yaw rate deviation is not considered when it is less than the threshold of the yaw rate deviation. For brake control input, the RMS braking torque $T_{b}$ of four wheels is assessed using the sum of the individual RMS braking torques at each wheel. This RMS braking torque can be interpreted loosely as the energy or control effort expended in controlling the vehicle motion.

The Sine-With-Dwell maneuver is performed with an initial vehicle speed $80 \mathrm{~km} / \mathrm{h}$ on a dry road (road surface friction 0.9 ), and the gear ratio of the handwheel steer is 1/16. It is assumed that all vehicle variables, braking torques, tire forces and the surface friction coefficient are available to the controller from direct measurements or appropriate estimations (Satria and Best, 2002; Wilkin et al., 2005) if there is no mention about practical measurements or estimation approaches.

\subsubsection{CONTROL SCENARIOS AND DESIGN PARAMETER TUNING}

\section{(i) Control scenarios}

Performance benefits for vehicle stability control based on yaw and sideslip motion control were evaluated for four different control scenarios with a $0.7 \mathrm{~Hz}$ SineWith-Dwell maneuver: 
Case 1 (No braking control): No VSC control is implemented to show free responses by an open loop steer input

Case 2 (Rule-based): VSC is implemented to address over-steer motions by applying or releasing braking force at the right or left wheel, and more details can be found in Appendix C

Case 3 (LTI-MPC): VSC is implemented using a pre-defined 3 DoF linear vehicle model with four wheel individual braking control

Case 4 (LTV-MPC): VSC is implemented using a 3 DoF nonlinear vehicle model with four wheel individual braking control (see Chapter 4).

Here Case 4 (LTV-MPC) covers the proposed controllers, FICC, the rule-based controller and the LTI-MPC controller are chosen as the bases for a comparison with the proposed controller. The rule-based controller is a simple design, even though the related control design rules can be developed or refined with a level of sophistication. More sophisticated design procedures can be expected with various intensive work loads. The LTV-MPC controller and the LTI-MPC controller use the same standard quadratic optimization solver (FSQP, details in Section 5.6.1.) to determine control inputs, except for the predictive model used for the intermediate layer of the VSC. In other words, the LTI-MPC controller uses a fixed linear predictive model for the MPC, whereas the LTVMPC controller uses a revised linearization model at each time step. As noted in Chapter 3, the LTI-MPC has a benefit from the computational load viewpoint in that it uses a preset linear model, but the LTI-MPC has difficulty reflecting the vehicle nonlinearity during severe transient maneuvers. On the other hand, the LTV-MPC can reflect the vehicle nonlinearity but this requires on-line numerical linearization.

\section{(ii) Control design parameter tuning}

Controller performance depends on the tuning objectives, and needs to balance between multiple objectives. Multiple objectives must be considered that the emphasis on one objective can cause other objectives to degrade. For example, aggressive 
handling responsiveness tracking can lead to handling stability problems in terms of vehicle spin-out.

In this study, the goal of controller tuning is to maintain the vehicle sideslip less than 5 degrees to meet the priority of handling stability, and then to minimize the yaw rate tracking error to address handling responsiveness. To achieve these controller tuning goals, controller tuning is achieved off-line using commercial optimization software, iSIGHT (iSight, 2004). A Sequential Quadratic Programming (NLPQL) algorithm in iSIGHT was used in order to minimize the cost function, where the cost function is defined as the RMS yaw rate tracking error with the sideslip constraint such as $\beta(t) \leq 5 \mathrm{deg}$. The controller tuning results are given in Table E.2 and Table E.3.

\subsection{PERFORMANCE EVALUATION}

In this section, controller performance is evaluated for an exact model-plant match, where the model represents a nominal vehicle model which is used for the control design and the plant is an actual vehicle system. The responses of the exact model-plant match is used as reference responses for more realistic situation such as model-plant mismatch due to uncertainty of the real plant. The controller performance assessment follows the SAE coordinate system (Gillespie, 1992).

In order to satisfy the criteria of a Sine-With-Dwell maneuver given in Eqs. (5.1) and (5.2), the maximum allowable peak handwheel steer $\left|\delta_{H W}\right|_{\text {peak }}$ is first investigated with a handwheel steer limit of 270 degrees. In other words, if necessary, a reduced peak handwheel steering angle is used to satisfy the stability criteria rather than using the handwheel steer limit proposed on the original Sine-With-Dwell maneuver. Vehicle responses are then evaluated in terms of the RMS (root mean square) yaw rate error $\left|e_{r}\right|_{R M S}$ to represent yaw rate tracking, peak sideslip $|\beta|_{\text {peak }}$ and braking control effort (RMS braking torque $T_{b}$ ). 


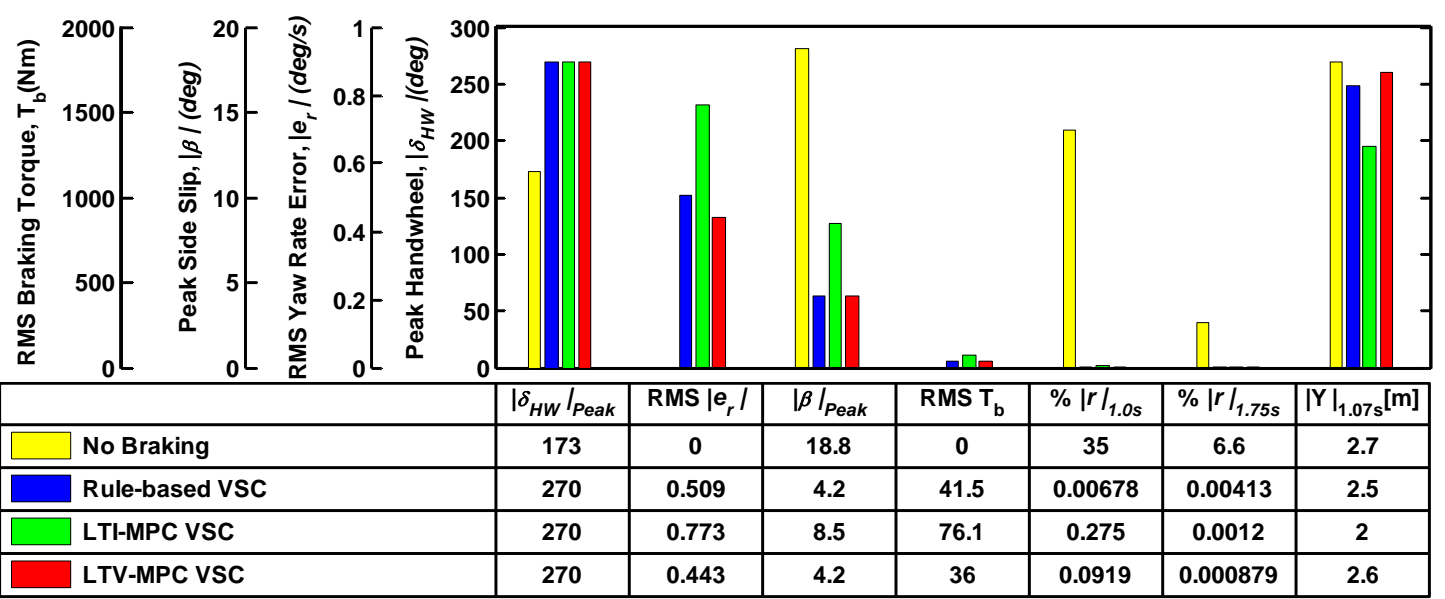

Fig. 5.3 Performance comparison for Sine-With-Dwell maneuver (8DoF, $U_{0}=80 \mathrm{~km} / \mathrm{h}, \mu_{H}=0.9$ )

(a) Yaw rate

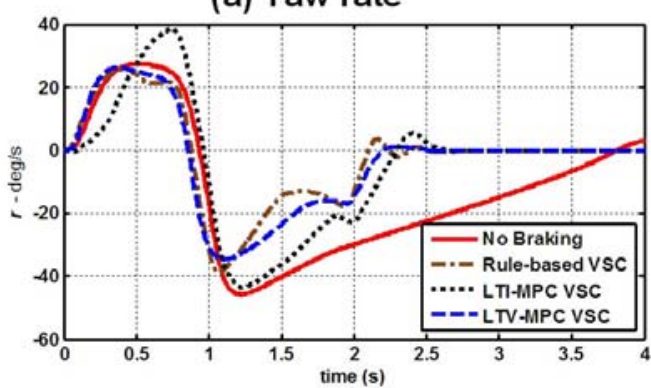

(b) Side slip

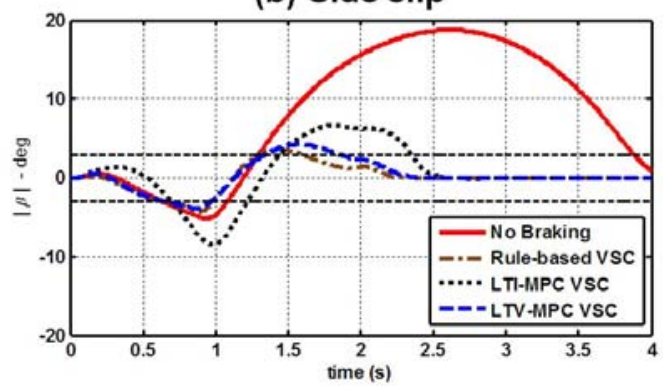

Fig. 5.4 Yaw rate and sideslip responses during Sine-With-Dwell maneuver (8DoF, $U_{0}=80 \mathrm{~km} / \mathrm{h}, \mu_{H}=0.9$ )

Fig. 5.3 shows peak handwheel steer, RMS yaw rate error, peak side slip, RMS braking torque, and yaw rate percent and lateral displacement defined by NHTSA criteria given in Eqs. (5.1) and (5.2). Figs. 5.4 and 5.5 present vehicle responses - yaw rate and sideslip, and wheel slip ratio and braking torque, respectively.

As shown in Fig. 5.4 (a) and (b), even though the peak handwheel steer of the no VSC vehicle is 173 degrees to satisfy NHTSA stability criteria, the no braking control responses show relatively large motions in terms of increased sideslip compared to those of VSC equipped vehicles with a limit handwheel steer of 270 degrees. The LTI-MPC 
(a) Slip ratio - Rule-based VSC

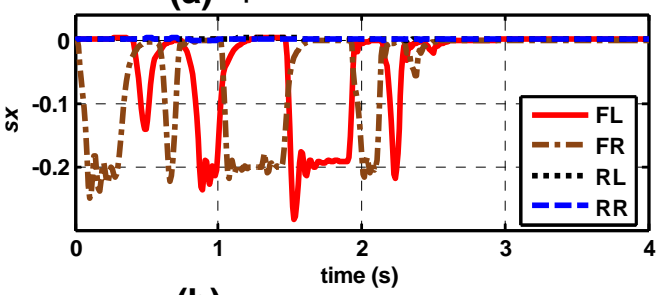

(b) Slip ratio - LTI-MPC vSC

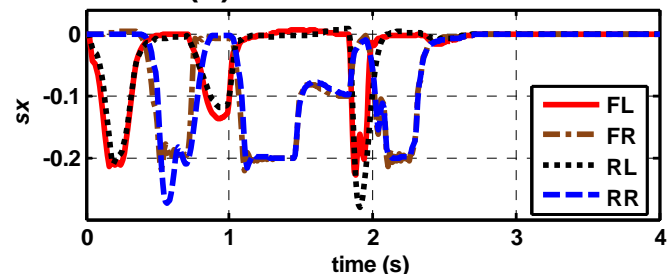

(c) Slip ratio - LTV-MPC VSC

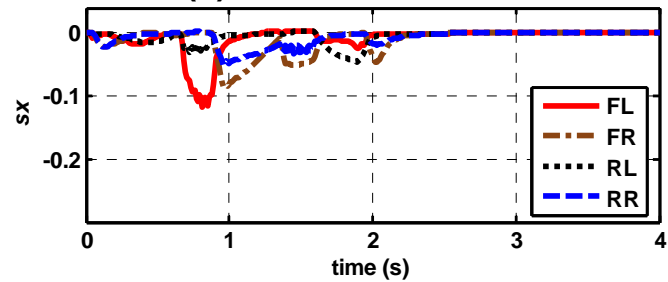

(d) Brake Torque - Rule-based VSC

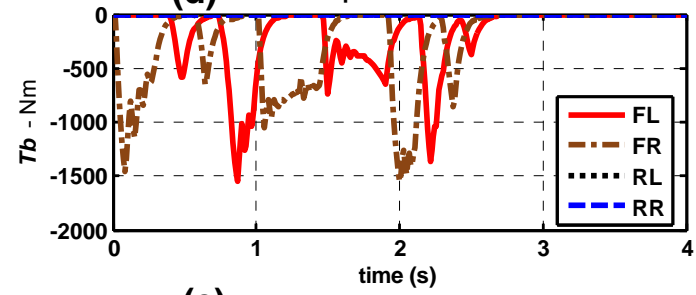

(e) Brake Torque - LTI-MPC vSC

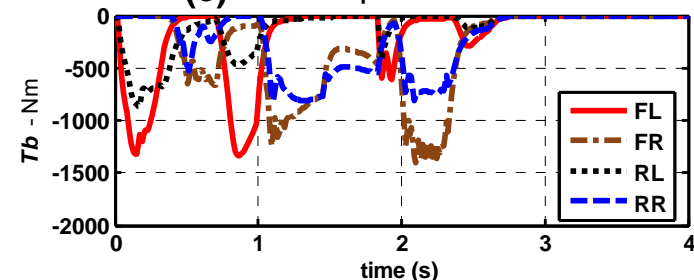

(f) Brake Torque - LTV-MPC VSC

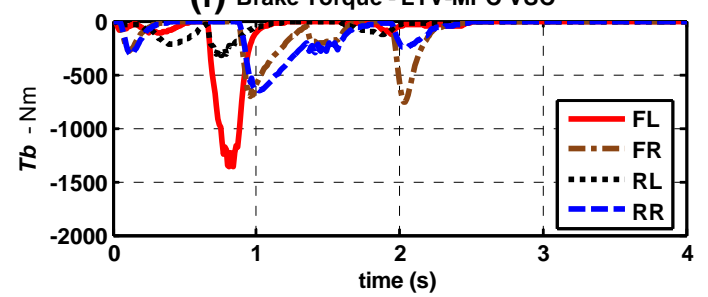

Fig. 5.5 Slip ratio responses (a-c) and braking torque responses (d-f) according to different controllers during Sine-With-Dwell maneuver $\left(8 \mathrm{DoF}, U_{0}=80 \mathrm{~km} / \mathrm{h}, \mu_{H}=0.9\right.$ )

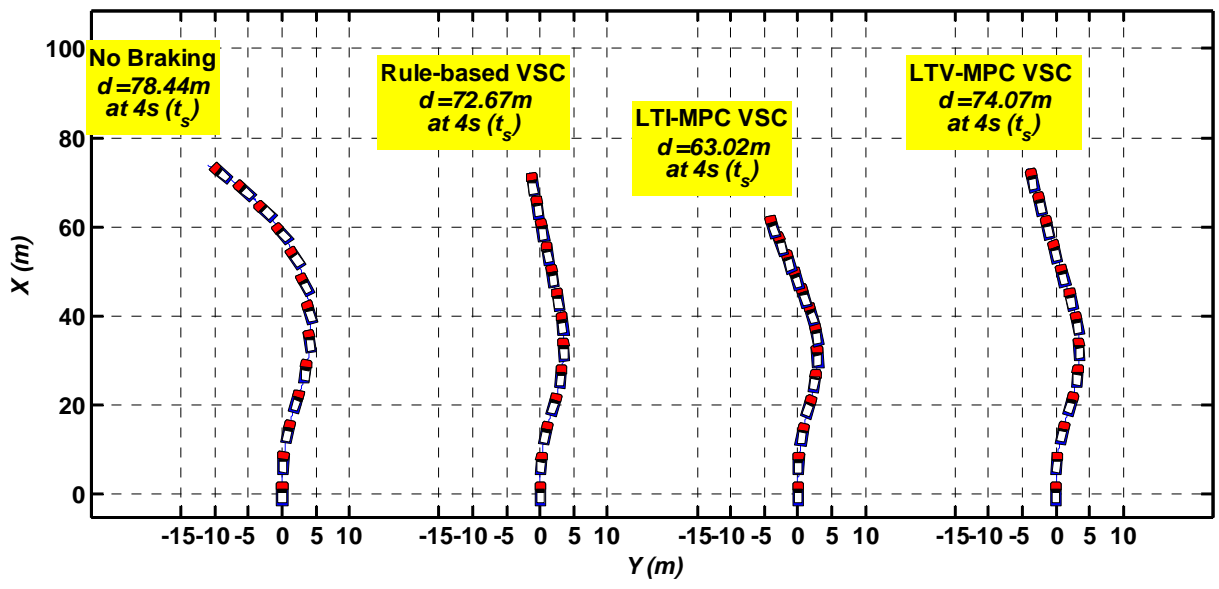

Fig. 5.6 Vehicle trajectories and moving distances $(d)$ for Sine-With-Dwell maneuver (8DoF, $U_{0}=80 \mathrm{~km} / \mathrm{h}, \mu_{H}=0.9$ )

controller shows the worst performance. The tuning procedure presented in Section 5.1.2 did not satisfy the sideslip constraint $\left(|\beta|_{\text {peak }}>5 \mathrm{deg}\right)$ as shown in shows Fig. 5.3. 
This failure likely occurred because the LTI-MPC based VSC overacted during the transient motions. This overact was due to the system's inability to consider the transient motions such as load transfer. Moreover, as shown in Fig. 5.5, the slip responses of front and rear wheels are almost identical to the compensation for the tracking error using the linear predictive model at the equilibrium points, while the slip responses of the LTV MPC-based VSC are different on the basis of the transient vehicle responses.

Figs. 5.5 (a) and (d) demonstrate that the rule-based VSC uses only front wheels to correct vehicle motions due to the pre-defined rule. In this condition, the peak braking torque is relatively larger than that of any other controller. The LTV-MPC controller, on the other hand, distributes braking torque progressively over four wheels on the basis of vehicle transient motions. Therefore, the maximum slip ratio and braking torque are significantly smaller than they are for other controllers.

With respect to moving distance during the simulation time 4 seconds, the larger braking force of the LTI-MPC controller leads to a shorter distance $(63.02 \mathrm{~m})$ traveled than that resulting from the LTV-MPC controller $(74.07 \mathrm{~m})$, as shown in Fig. 5.6.

\subsection{VSC ROBUSTNESS}

As mentioned above, the LTV-MPC VSC requires state and model parameter information in order to function. The question arises as to whether the LTV-MPC VSC is reasonably robust to errors, especially in terms of model-plant mismatch. Thus the brake-based VSC controller designed for an exact model-plant match is first investigated. Further investigation of controller robustness is performed by relaxing the following assumptions: various road and speed conditions, a model-plant mismatch, different actuator time constraints, vehicle state and tire force estimation, and road surface friction estimation. 

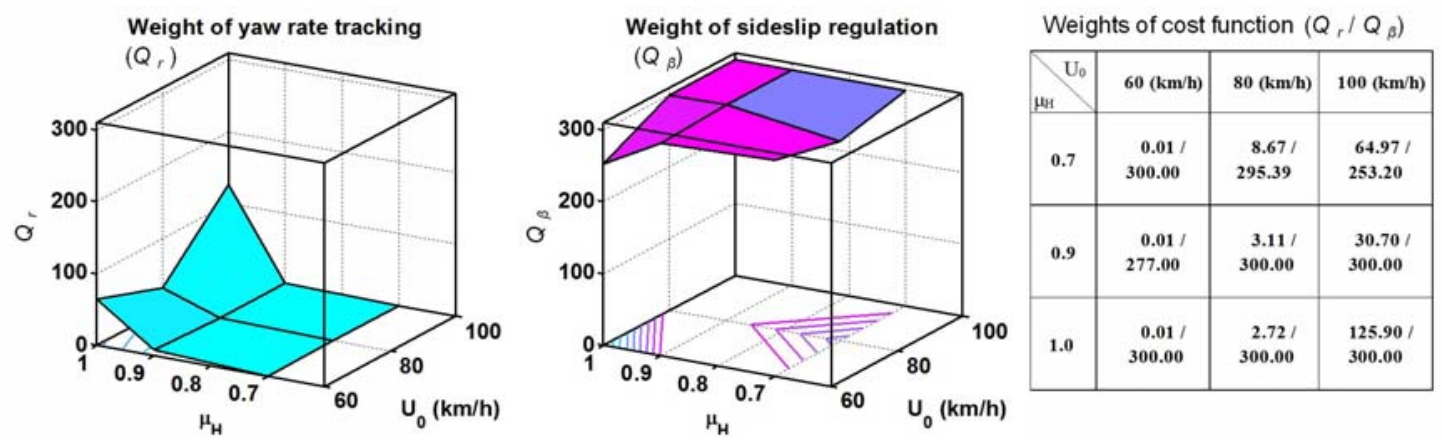

Fig. 5.7 Retuned weighs of cost function for different driving conditions w.r.t. road surface friction and initial vehicle speed (8 DoF)

\subsubsection{MODEL-PLANT MISMATCH}

The exact model-plant match is simulated using an 8 DoF vehicle model along with a brake-based LTV-MPC controller. The proposed controller uses a 3 DoF nonlinear vehicle model having the same vehicle parameters and nonlinear tire model which are used for the plant, an 8 DoF vehicle model described in Appendix A. In contrast to the exact model-plant match, the model-plant mismatch consists of a CarSim vehicle model and the brake-based LTV-MPC controller based on the 8 DoF vehicle model.

\section{(i) Controller design parameter tuning}

The metrics of Sine-With-Dwell maneuver conditions consist of 9 different driving conditions including 3 different road surface frictions $(0.7,0.9,1.0)$ and 3 different vehicle initial speeds $(60 \mathrm{~km} / \mathrm{h}, 80 \mathrm{~km} / \mathrm{h}, 100 \mathrm{~km} / \mathrm{h})$.

Fig. 5.7 shows the retuned weights of the cost function for each driving condition. The weights of sideslip show nearly the highest value under the fixed maximum limit of the sideslip weight, which is aimed to investigate the relative scale between the sideslip weight and yaw rate weight. This means that in order to minimize yaw rate tracking error the controller tends to minimize the sideslip regulation activation because the control priority is imposed on the sideslip regulation. The magnitude of the retuned yaw rate 


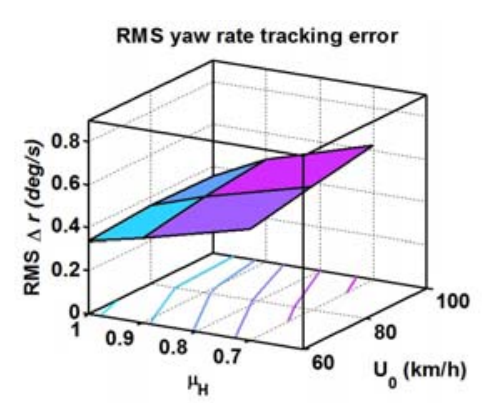

$\max (0.623)$, mean ( 0.4529), std( 0.1012$)$

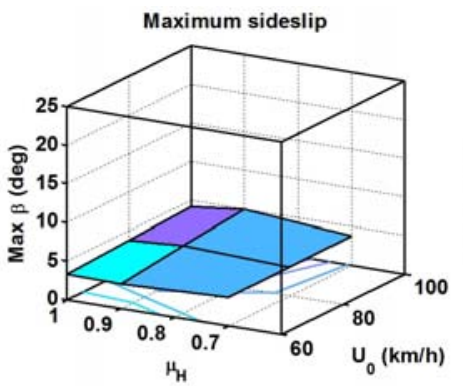

$\max (5.0)$, mean ( 3.9), std( 0.5)

(a)

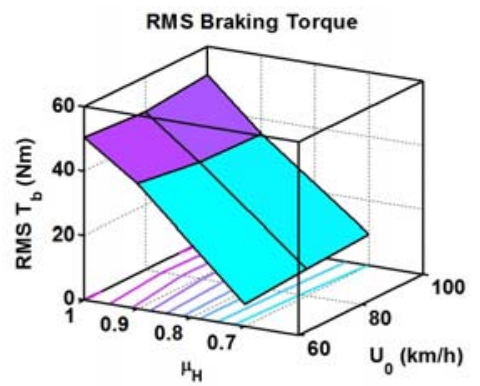

$\max (50.9)$, mean (31.7), std( 18.6)

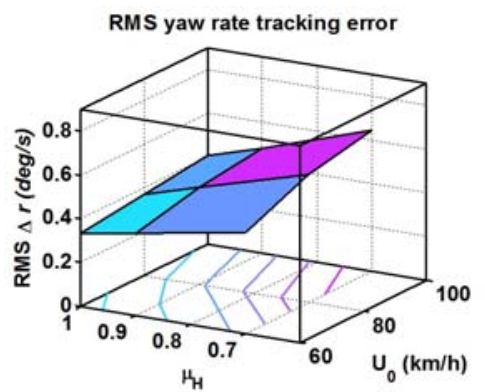

$\max (0.643), \operatorname{mean}(0.4557), \operatorname{std}(0.1022)$

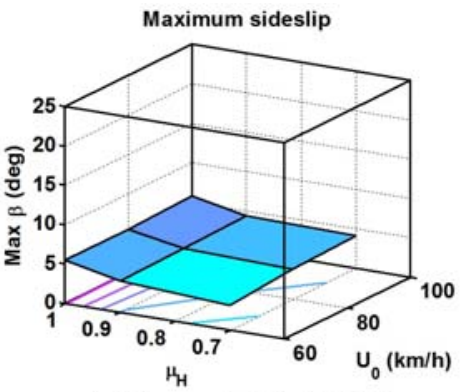

$\max (5.7)$, mean (4.5), $\operatorname{std}(0.9)$

(b)

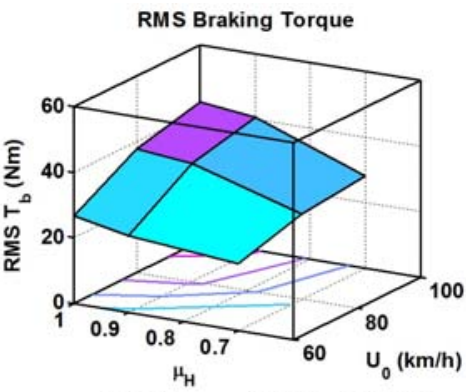

$\max (42.4)$, mean( 31.3), std( 8.0)

Fig. 5.8 Performance metrics of (a) retuned weights of cost function and (b) fixed weights of cost function for the exact model-plant match (8DoF)

weight is proportional to the vehicle motion severity. For example, the highest yaw rate weight occurs under the high road fiction and high speed.

\section{(ii) Performance comparison between Retuned-weight LTV-MPC vs. Fixed- weight LTV-MPC}

The purpose of this section is to investigate the effect of the pre-fixed weight of the cost function on the MPC design because the retuned weights do not mean the best tuning practically due to the model-plant mismatch even though the retuned weight on the model-plant match can be scheduled using a look-up table form.

The fixed-weight LTV MPC-based VSC uses control design parameters which are tuned at $\mu_{H}=0.9$ and $U_{0}=80 \mathrm{~km} / \mathrm{h}$. 

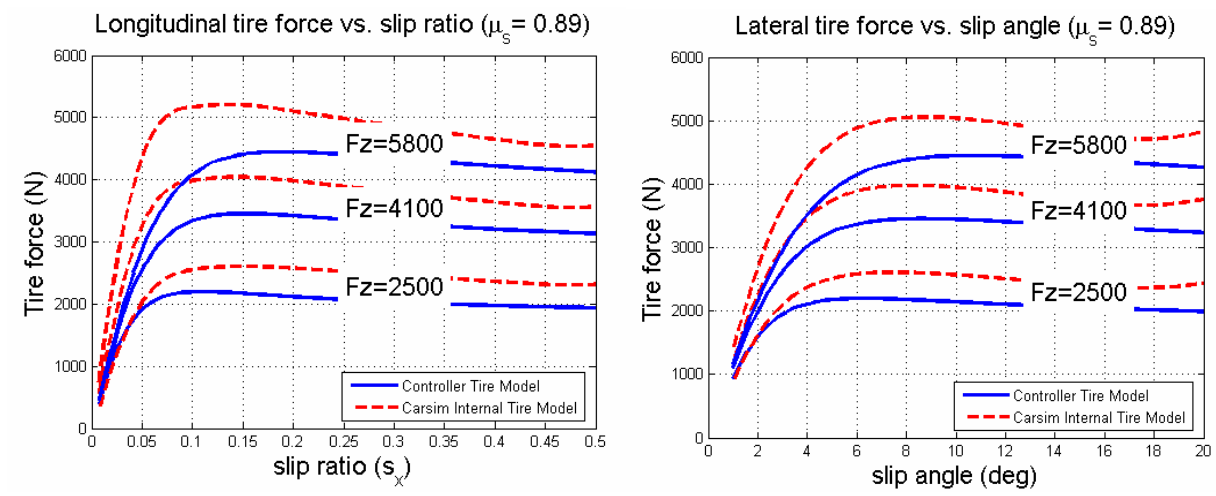

Fig. 5.9 Comparison between CarSim and 8 DoF tire models

Fig. 5.8 shows a slight increase in the fixed-weight LTV-MPC for yaw rate tracking error and maximum sideslip in terms of maximum value, mean value and standard deviation of 9 cases. This slight degradation of the LTV-MPC control performance likely occurs due to the reduced braking torque compared to the retunedweight LTV-MPC.

\section{(iii) Model-plant mismatch}

A CarSim vehicle model was used to examine the effect of mismatch between the actual vehicle and the control model used for MPC. Vehicle and tire modeling errors result from the implementation of the exact same controller within the CarSim model, which has a different structure and in particular has quite different tire characteristics- see Fig. 5.9.

Even though the control design parameters tuned for the 8 DoF vehicle model is implemented without retuning for the CarSim model, the results of the CarSim model follow trends similar to those of the 8 DoF vehicle model for different driving maneuvers. Fig. 5.10 shows that the results for the CarSim model parallel those of the 8 DoF results presented in Fig. 5.8. Vehicle yaw and sideslip responses are only very slightly changed in the CarSim model, though braking torque actuation is of larger amplitude. The existence of slight different responses is hardly surprising - the CarSim 


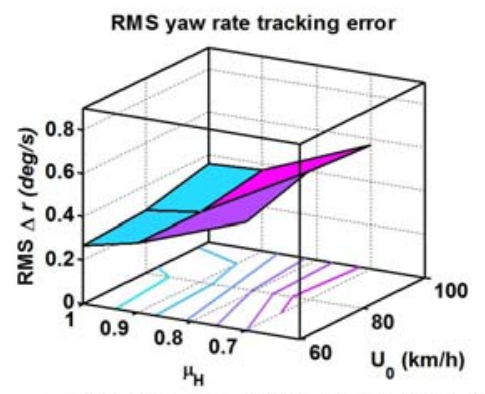

$\max (0.589)$, mean ( 0.3987), $\operatorname{std}(0.1222)$

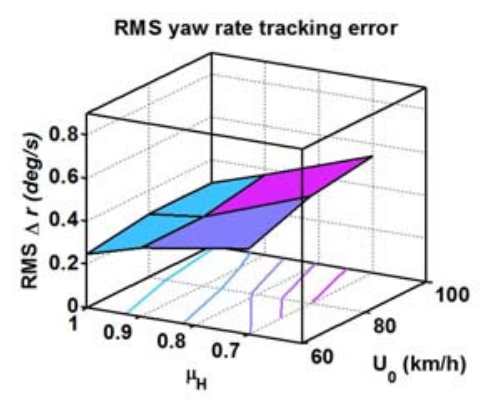

$\max (0.540)$, mean $(0.3646), \operatorname{std}(0.0986)$

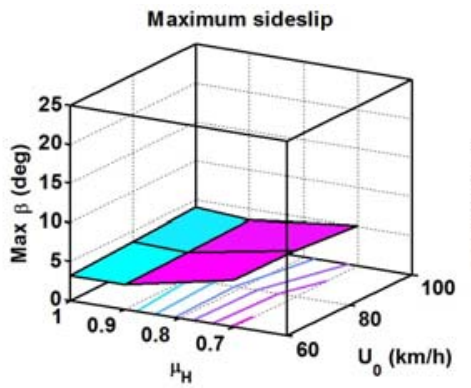

$\max (6.1)$, mean( 4.2), $\operatorname{std}(1.1)$

(a)

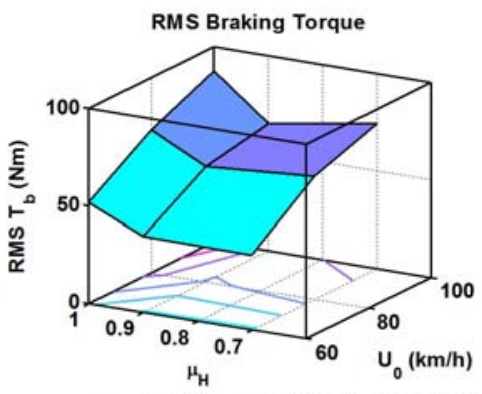

$\max (87.8)$, mean( 61.3), std( 16.4)

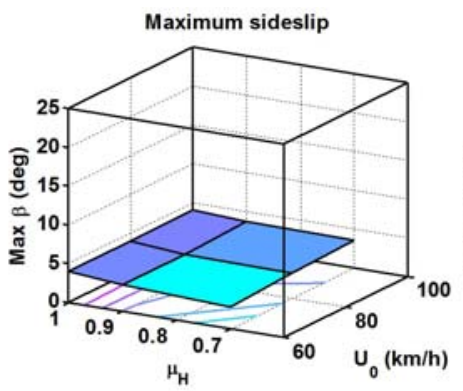

$\max (4.1)$, mean (3.6), std( (0.4)

(b)

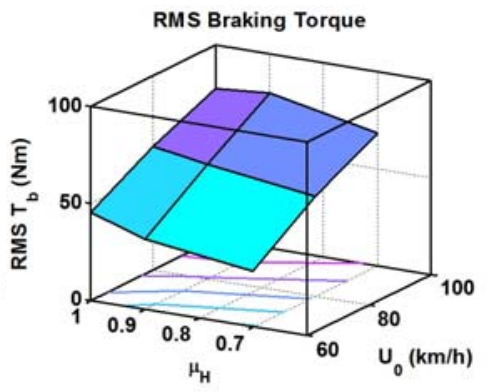

$\max (79.9), \operatorname{mean}(56.7), \operatorname{std}(17.8)$

Fig. 5.10 Performance metrics of (a) retuned weights of cost function and (b) fixed weights of cost function for the model-plant mismatch (CarSim)

model has very different suspension characteristics compared to the simpler 8 DoF model, as well as very different tire characteristics.

As shown in Fig. 5.10, the retuned-weight controller does not guarantee better performance over the fixed-weight controller in the model-plant mismatch. However, the performance of each controller is still similar to that of the exact model-plant mismatch except for the increased braking torque usage that seems to result from the tire model difference.

\subsubsection{ACTUATOR TIME CONSTANT}

The effects of the actuator time constants are investigated for the braking control as a stand-alone low level controller and the brake-based vehicle stability control as an integrated controller. 


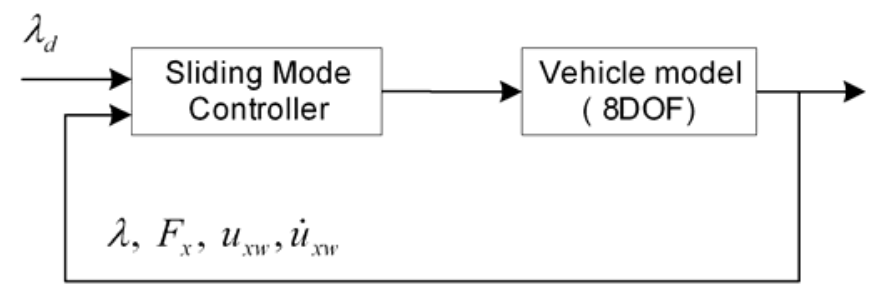

Fig. 5.11 Block diagram of actuator time constant test using wheel slip controller

\section{(i) Wheel slip control}

In order to assess the effects of the actuator time constants on controller performance, the wheel slip controller is investigated through simulation. The wheel slip is well suited for this analysis because the wheel slip control is the lowest level control. The design parameters of the wheel slip controller are tuned off-line using commercial optimization software, iSIGHT (iSight, 2004), with a Sequential Quadratic Programming (NLPQL) algorithm. The cost function is defined as total tracking error which is the summation of the absolute error between the target slip ratio and actual slip ratio at four wheels during simulation. The smaller cost function can be interpreted as less chatter (oscillation) around the target slip ratio, where less chatter reduces the brake load as well as increases the durability of brake hardware. Among the design parameters needed for robust wheel slip controller design, a sliding surface design parameter $\gamma$ and a switching gain design parameter $\eta$ are optimized to minimize the slip ratio tracking error with preselected values for the rest of the design parameters. The design parameter optimization and simulation analysis are performed by using the ideal full state feedback model shown in Fig. 5.11. Pure braking tests in an open-loop straight-line maneuver are performed at the initial velocities, $120 \mathrm{~km} / \mathrm{h}\left(\mu_{H}=0.9\right)$.

The actuator dynamics generally affects the performance of the SMC by delaying responses or increasing the oscillation in transient responses due to the physical limits of 


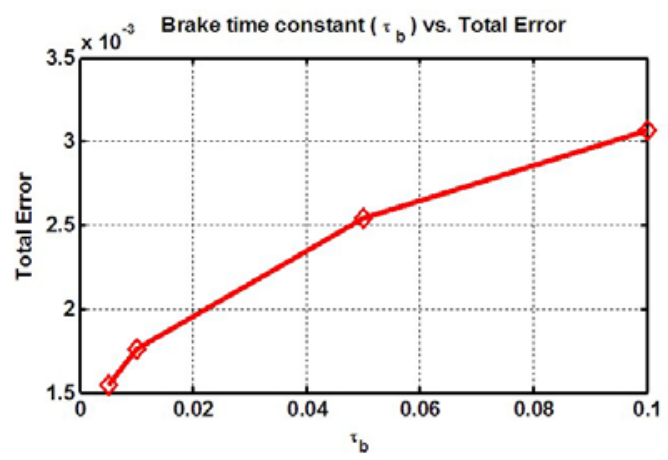

\begin{tabular}{|c|c|c|c|c|c|c|}
\hline \multirow{3}{*}{$\frac{\tau_{\mathrm{b}}}{}$} & \multicolumn{5}{|c|}{ Design Parameters } & \multirow{3}{*}{$\begin{array}{l}\begin{array}{l}\text { Total } \\
\text { Error }\end{array} \\
0.00154\end{array}$} \\
\hline & \multicolumn{2}{|c|}{$\eta(\mathrm{Fr} / \mathrm{Rr})$} & \multicolumn{2}{|c|}{ Y $(\mathrm{Fr} / \mathrm{Rr})$} & \multirow{2}{*}{$\frac{\Phi}{52225}$} & \\
\hline & 0 & 0 & 208.9 & 182.7 & & \\
\hline 0.01 & 0 & 0 & 135.8 & 129.9 & 3.395 & 0.00176 \\
\hline 0.05 & 0 & 0 & 103.4 & 103.1 & 2.585 & 0.00254 \\
\hline 0.1 & 0 & 0 & 121.6 & 1148 & 3.04 & 0.00306 \\
\hline
\end{tabular}

Fig. 5.12 Comparisons of total tracking error for target slip ratio with varying brake time constants $\left(\lambda_{d, f}=-0.12, \lambda_{d, r}=-0.1, \mu_{H}=0.9,8 \mathrm{DoF}\right)$

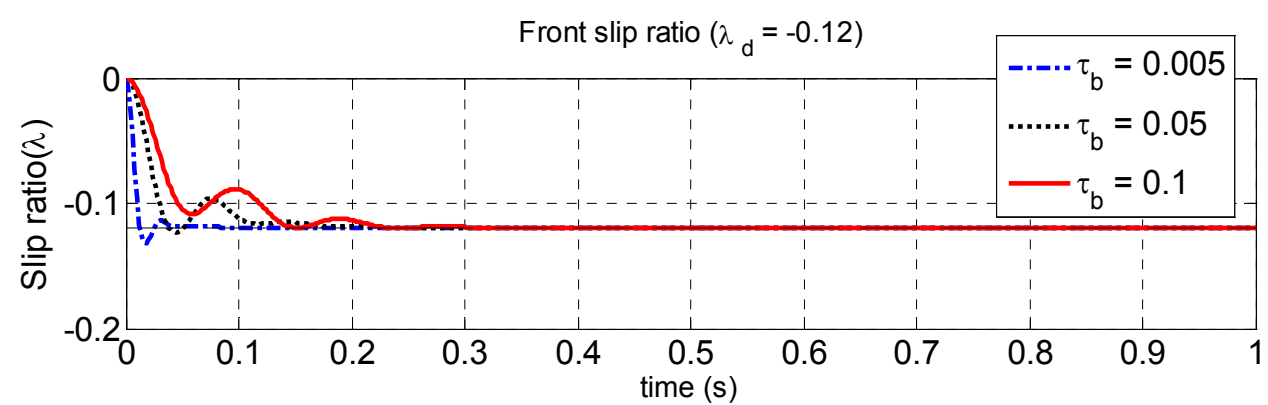

Rear slip ratio $\left(\lambda_{d}=-0.1\right)$

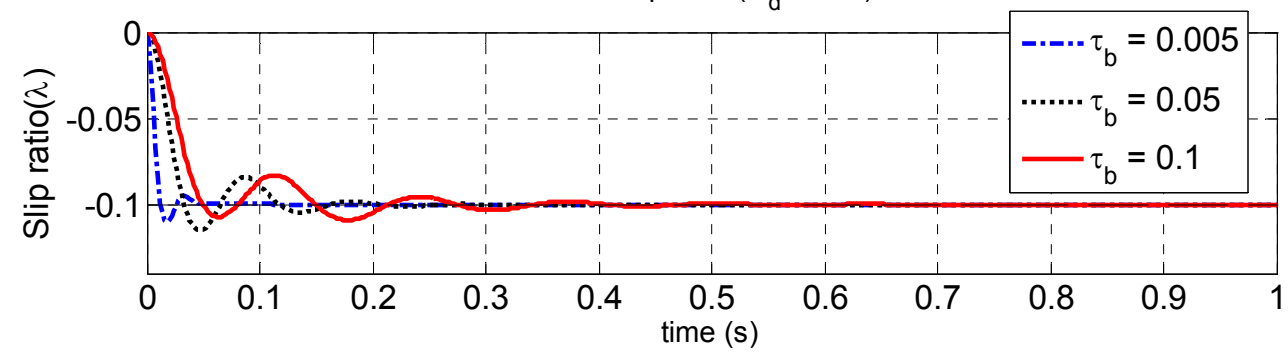

Fig. 5.13 Comparisons of target slip ratio tracking with varying brake time constants $\left(\lambda_{d, f}=-0.12, \lambda_{d, r}=-0.1, \mu_{H}=0.9,8 \mathrm{DoF}\right)$

the actuators. The brake actuator modeled is a first-order system, whose time constant affects the target slip ratio tracking performance. This effect is investigated with off-line tuned optimal design parameters.

As shown in Figs. 5.12 and 5.13, increasing the brake time constant causes the wheel slip tracking error and the oscillation around target slip ratio to be large due to actuator delay. 

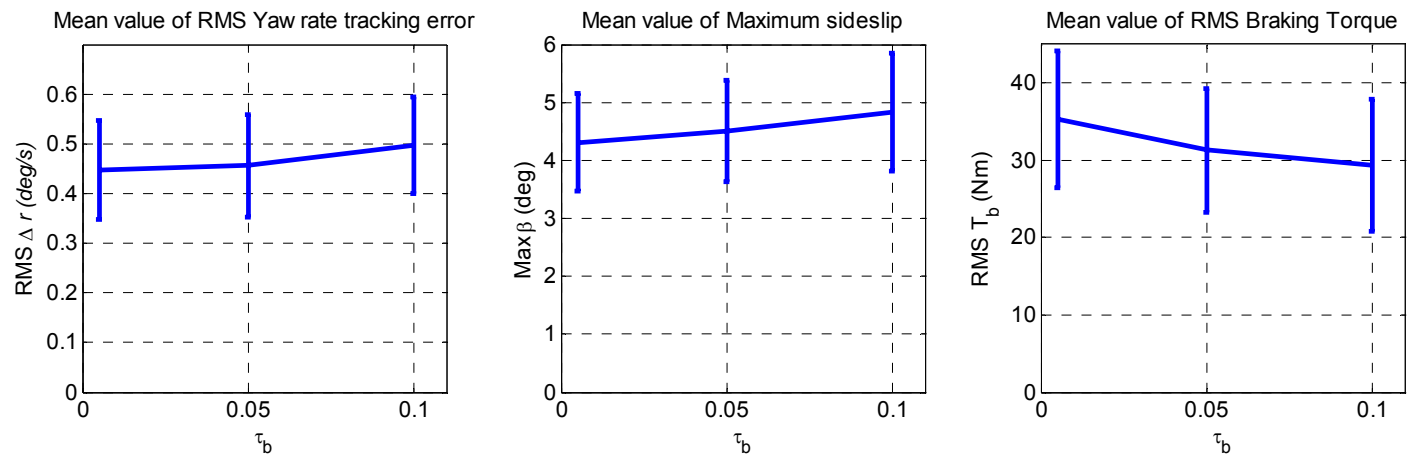

Fig. 5.14 Performance summary of the fixed-weight LTV-MPC VSC for different brake time constants in terms of mean and standard deviation of 9 different driving conditions during Sine-With-Dwell maneuver(8DoF model)

\section{(ii) Brake-based VSC}

At the integrated control level, the effect of the brake time constant is investigated. The simulation is achieved using the $8 \mathrm{DoF}$ model and the fixed-weight LTV-MPC VSC for different brake time constants during the Sine-With-Dwell maneuver. The performance is summarized in terms of mean and standard deviation of 9 different driving conditions including 3 different road surface frictions $(0.7,0.9,1.0)$ and 3 different vehicle initial speeds $(60 \mathrm{~km} / \mathrm{h}, 80 \mathrm{~km} / \mathrm{h}, 100 \mathrm{~km} / \mathrm{h})$. In Fig. 5.14 , mean value corresponds to the center point of the vertical bar, and standard deviation is the distance from the center point to the end point of the vertical bar.

As shown in Fig. 5.14, the fast actuator (small time constant) provides more sophisticated control performance by providing smaller RMS yaw rate tracking error and maximum sideslip compared to other braking control with relatively large brake time constants. Because of this effect, actuator should be chosen by considering its performance and hardware cost because the actuator hardware cost is proportional to its performance.

\subsubsection{VEHICLE STATE ESTIMATION}


Table 5.1 Sensor noise characteristics in terms of standard deviation, $\sigma$

\begin{tabular}{cc}
\hline Accelerometer & $0.1 \mathrm{~m} / \mathrm{s}^{2}$ \\
\hline Wheel speed sensor & $0.03 \mathrm{~m} / \mathrm{s}(5.7 \mathrm{deg} / \mathrm{s})$ \\
\hline Yaw rate gyro sensor & $0.3 \mathrm{deg} / \mathrm{s}$ \\
\hline Brake torque sensor & $21 \mathrm{Nm}$ \\
\hline Steering wheel angle sensor & $2.5 \mathrm{deg}$ \\
\hline
\end{tabular}

In this section, the effects of estimation are investigated by relaxing the assumptions that all vehicle variables, braking torques, tire forces and the surface friction coefficient are available. The estimation of vehicle states and tire forces is based on the Extended Kalman Filter (EKF) in this study. The EKF is designed by following the procedure presented in (Ray, 1997), and a brief summary of its procedures for the 8 DoF nonlinear vehicle model is shown below. The state and measurement equations are

$$
\dot{\vec{x}}_{A}=f\left(\vec{x}_{A}, \vec{u}\right) \text { and } \vec{y}=h\left(\vec{x}_{A}, \vec{u}\right)
$$

The augmented nonlinear state equation consists of the tire force term $\left(\dot{F}_{x, y}=\dot{F}_{x, y}\right.$ and $\left.\ddot{F}_{x, y}=0\right)$ and the $8 \mathrm{DoF}$ vehicle dynamics given in Appendix A. The augmented state vector with approximation symbol " $\wedge$ " is constituted by nine vehicle states, six estimated tire forces and six first derivatives of estimated forces.

$$
\begin{gathered}
\vec{x}_{A}(1: 9)=\left[\hat{U}, \hat{V}, \hat{\Omega}, \hat{\varphi}, \hat{\dot{\varphi}}, \hat{\omega}_{1}, \hat{\omega}_{2}, \hat{\omega}_{3}, \hat{\omega}_{4}\right]^{T} \\
\vec{x}_{A}(10: 15)=\left[\hat{F}_{x 1}, \hat{F}_{x 2}, \hat{F}_{x 3} \hat{F}_{x 4},\left(\hat{F}_{y 1}+\hat{F}_{y 2}\right),\left(\hat{F}_{y 3}+\hat{F}_{y 4}\right)\right]^{T} \\
\vec{x}_{A}(16: 21)=\left[\hat{\dot{F}}_{x 1} \hat{\dot{F}}_{x 2}, \hat{\dot{F}}_{x 3} \hat{\dot{F}}_{x 4},\left(\hat{\dot{F}}_{y 1}+\hat{\dot{F}}_{y 2}\right),\left(\hat{\dot{F}}_{y 3}+\hat{\dot{F}}_{y 4}\right)\right]^{T}
\end{gathered}
$$

Input vectors are composed of the steering angles and brake torques at each wheel shown in (5.5).

$$
\vec{u}=\left[u_{1}, u_{2}\right]
$$

where $u_{1}=\left[\delta_{1}, \delta_{2}, \delta_{3}, \delta_{4}\right]^{T}$ and $u_{2}=\left[T_{b 1}, T_{b 2}, T_{b 3}, T_{b 4}\right]^{T}$

For the output equation, the measurements of longitudinal and lateral acceleration, yaw rate, and roll angle at the c.g, and each wheel angular velocity are used. 


$$
\vec{y}=\left[a_{x}, a_{y}, \Omega, \varphi, \omega_{1}, \omega_{2}, \omega_{3}, \omega_{4}\right]^{T} .
$$

The system noise Q was chosen accordingly by comparing the relative magnitude order of $\mathrm{Q}$ with corresponding noise covariance in order to obtain estimation accuracy and robustness under model uncertainty. Particularly, the covariance for tire force term was set at large values to consider fast tire force change during transient motion (Ray, 1997; Wilkin et al., 2005). Noise covariance in Table 2 was determined by assuming uniform noise distribution with standard deviation given in the reference (Ryu et al., 2000; Bevly, 2004; Kiencke and Nielsen, 2005).

Using the estimated states, vertical tire force and slip ratio at each wheels are interpreted using the static equilibrium equation and kinematics in Eqs. (A.6) and (A.7), respectively. The longitudinal acceleration at each wheel $\dot{u}_{x w i}(i=1 \sim 4)$ for wheel slip control is approximated using following kinematic equations based on the vehicle states and geometry.

$$
\hat{\dot{u}}_{w i}=\hat{\dot{u}}_{i} \cos \delta_{i}+\hat{\dot{v}}_{i} \sin \delta_{i}(i=1 \sim 4)
$$

where

$\hat{\dot{u}}_{i}=\dot{U}+l_{u, i} \cdot \Omega^{2}=\left(a_{x}+V \cdot \Omega\right)+l_{u, i} \cdot \Omega^{2}, l_{u, i} \in\left[-L_{a},-L_{a}, L_{b}, L_{b}\right]$

$\hat{\dot{v}}_{i}=\dot{V}+l_{v, i} \cdot \Omega^{2}=\left(a_{y}-U \cdot \Omega\right)+l_{v, i} \cdot \Omega^{2}, l_{v, i} \in\left[\frac{t_{f}}{2},-\frac{t_{f}}{2}, \frac{t_{r}}{2},-\frac{t_{r}}{2}\right]$.

For simplicity, the estimation of other terms in the vehicle dynamics equation such as vehicle geometry data was considered as constant.

\section{(i) Vehicle state estimation results}

The purpose of this section is to investigate the effect of the state estimation, rather than achieve accurate state estimation, even though the EKF can be tuned to reduce the estimation error by increasing the accuracy of the nonlinear model or adding more measurements such as longitudinal and lateral speeds. In this study, the longitudinal and lateral speeds were estimated as shown in Fig. 5.15, and were used to calculate wheel slip, vehicle sideslip and other required states for the controller. Fig. 5.15 gives constant 

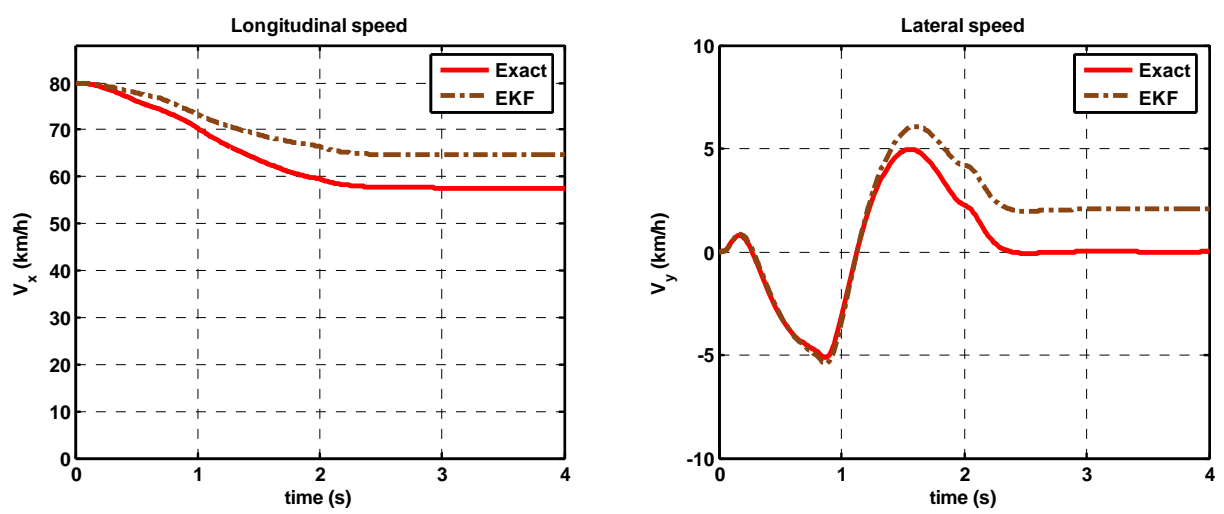

Fig. 5.15 Vehicle longitudinal and lateral speed estimation using EKF during Sine-WithDwell maneuver of LTV-MPC VSC (CarSim, $U_{0}=80 \mathrm{~km} / \mathrm{h}, \mu_{H}=0.9$ )
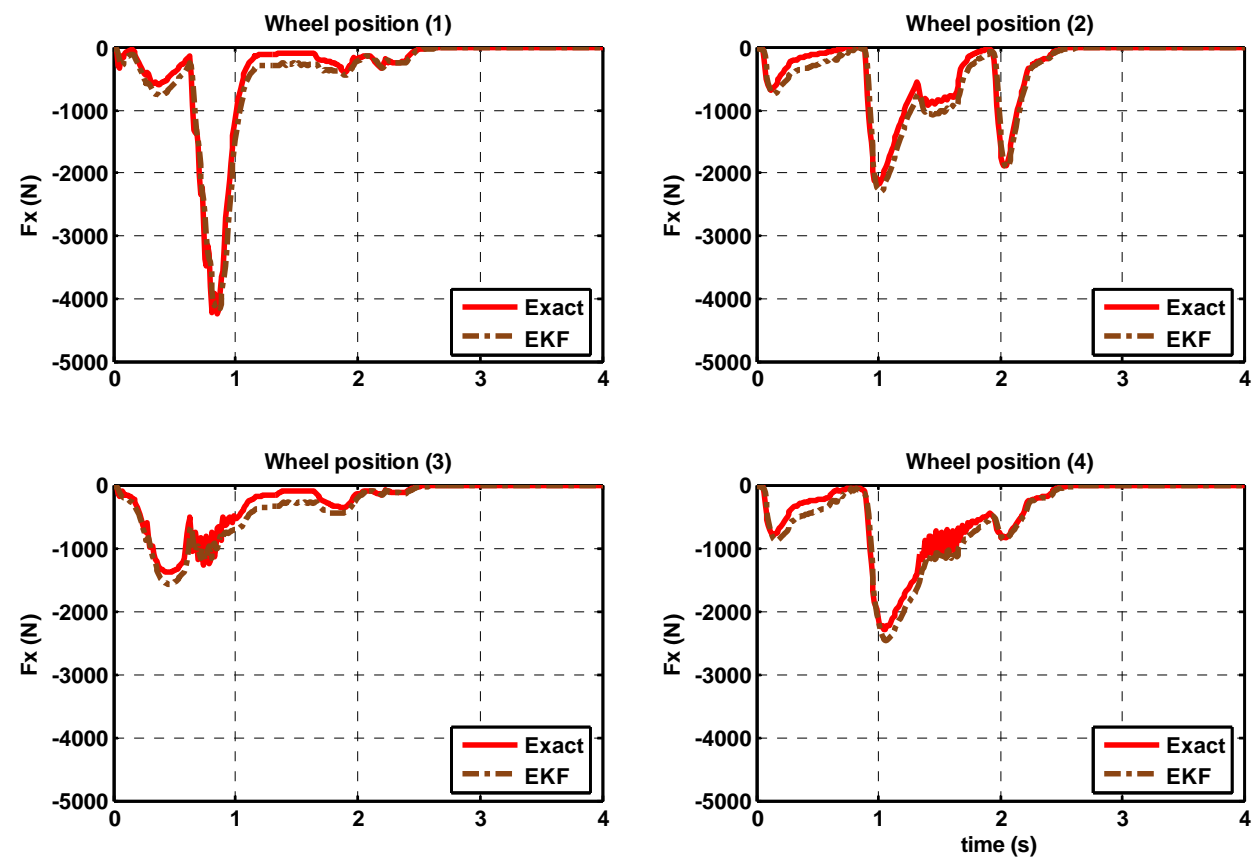

Fig. 5.16 Longitudinal tire force estimation using EKF during Sine-With-Dwell maneuver of LTV-MPC VSC (CarSim, $U_{0}=80 \mathrm{~km} / \mathrm{h}, \mu_{H}=0.9$ )

steady state estimation errors of longitudinal and lateral speeds. These errors are due to insufficient persistent excitation after the maneuver; in other words, there is no steer input and braking control after the maneuver because the constant sideslip estimation error is less than its threshold (approximated steady state value $2 \mathrm{deg}<3 \mathrm{deg}$ in this study). 


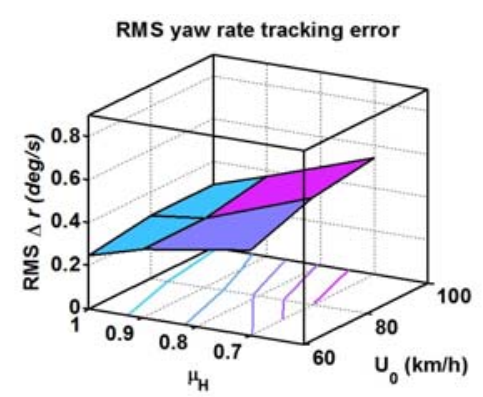

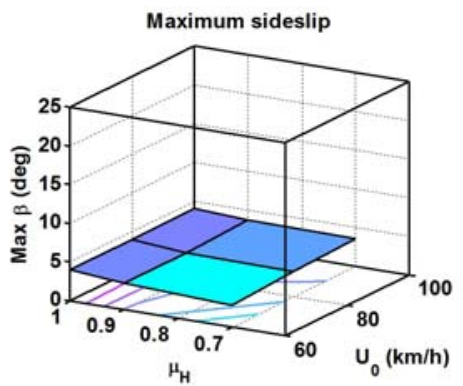

$\max (4.1)$, mean (3.6), std( 0.4)

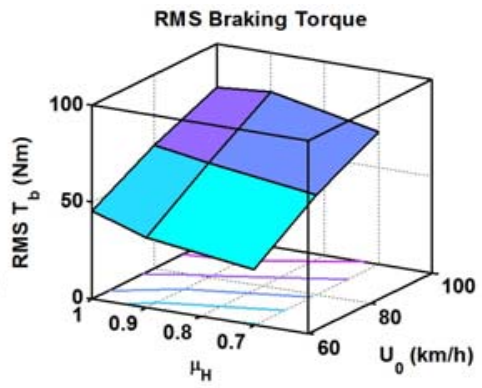

$\max (79.9)$, mean ( 56.7), std( 17.8)

(a) exact measurement (no noise) and known state assumption (CarSim)

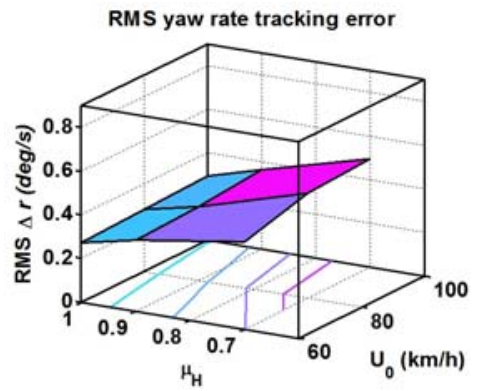

$\max (0.495), \operatorname{mean}(0.3616), \operatorname{std}(0.0822)$

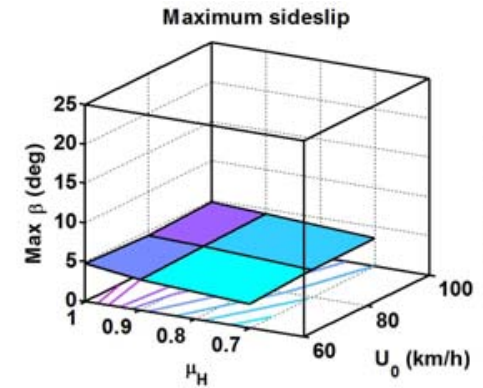

$\max (4.8)$, mean (4.0), std( 0.6)

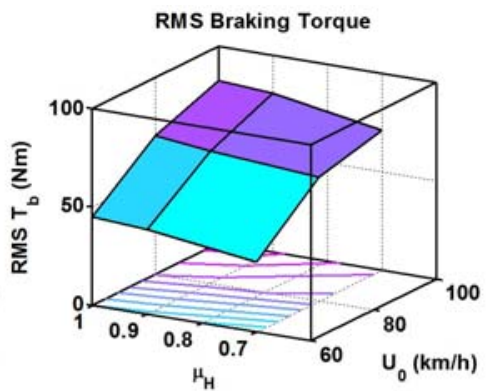

$\max (82.7)$, mean( 62.2), std( 17.1)

(b) state estimation and nosed measurement (CarSim)

Fig. 5.17 Performance metrics of (a) the fixed-weight LTV-MPC with no noise and known state assumption and (b) the fixed-weight LTV-MPC with EKF estimation during Sine-With-Dwell maneuver

In this situation, the controller does not work after the maneuver. One solution to address this steady state estimation error is using additional longitudinal or lateral speed measurement using the integration of inertial sensors with GPS (Bevly, 2004).

In Fig. 5.16, the estimated longitudinal tire force, which is used for the wheel slip control, is compared to the exact known values from the vehicle plant model. At the beginning of the maneuver, the estimation seems to work well but during severe transient maneuvers the estimation shows slightly a large deviation from the exact value.

\section{(ii) Performance comparison of the VSC between exact measurement vs. EKF}

In order to prevent the reference vehicle motion from being too sensitive against measurement noise, the reference yaw rate $r_{d}$ given in (3.2) is modified by a first order transfer function filter with $\tau_{f}=0.05$ 


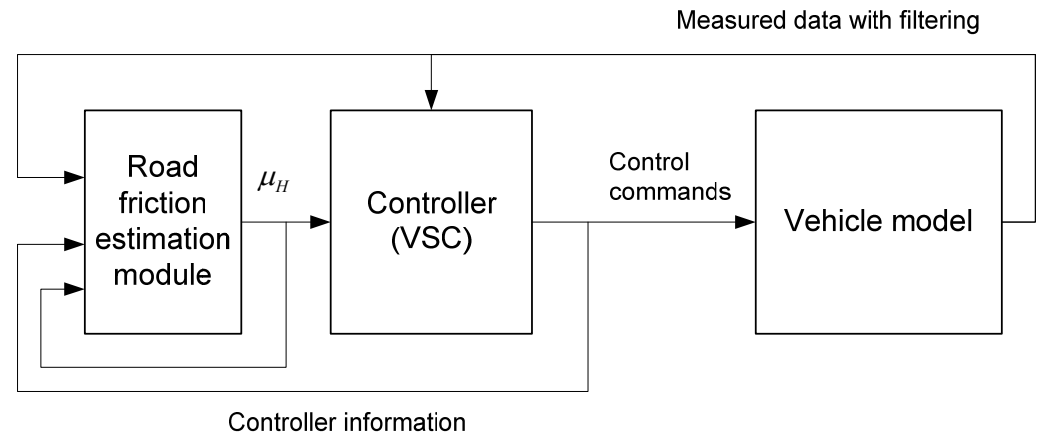

Fig. 5.18 Overall procedure of road surface friction estimation

$$
\frac{d r_{d}}{d t} \approx \frac{r_{d \text { filtered }}-r_{d}}{\tau_{f}}
$$

Here $r_{d \text { filtered }}$ is a filtered reference yaw rate. The other values for the controller such as wheel slip or vehicle sideslip are calculated using the estimated states by EKF.

Fig. 5.17 shows the performance metrics for the Sine-With-Dwell maneuver consisting of 9 different driving conditions such as 3 different road surface frictions $(0.7$, $0.9,1.0)$ and 3 different vehicle initial speeds $(60 \mathrm{~km} / \mathrm{h}, 80 \mathrm{~km} / \mathrm{h}, 100 \mathrm{~km} / \mathrm{h})$. The controller performance with state estimation is similar to that of the exact known state case, even though the sideslip and braking torque are slightly increased.

\subsubsection{ROAD SURFACE FRICTION COEFFICIENT ADAPTATION}

This section investigates the effect of the road surface friction mismatch on the controller and a vehicle plant. This investigation is important because the road surface friction plays a key role determining reference vehicle motions in terms of the saturation of the desired motions based on a simple bicycle model. Therefore, road surface friction is used to represent the physical limits of the desired motions of the vehicle stability controller such as yaw rate and deceleration, as shown in Fig. 5.18.

\section{(i) VSC performance without road surface friction adaptation}




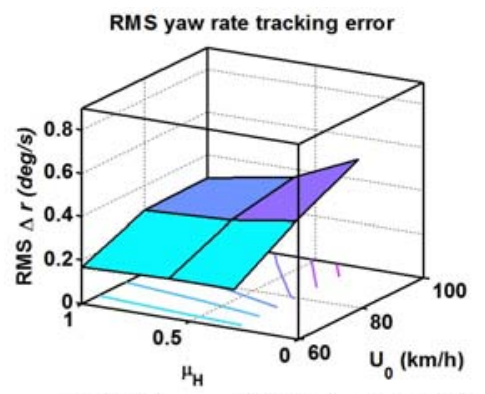

$\max (0.495), \operatorname{mean}(0.2926), \operatorname{std}(0.1068)$

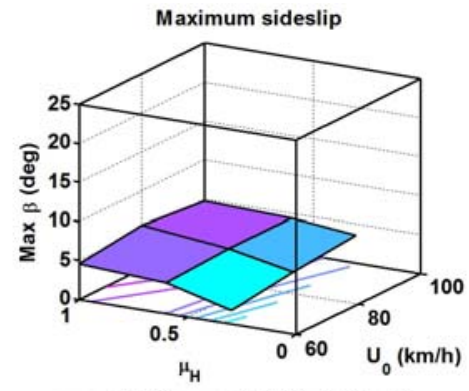

$\max (5.6)$, mean $(4.0), \operatorname{std}(1.1)$

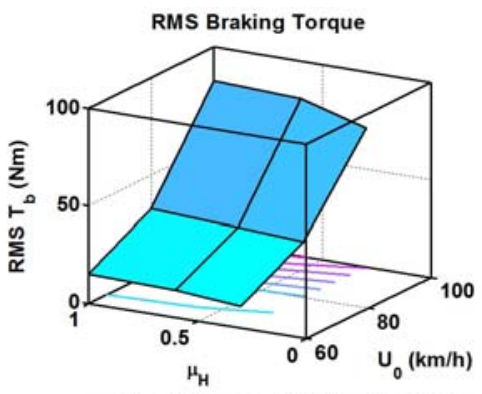

$\max (82.7)$, mean (40.8), std( 29.3)

(a) Exact known road surface friction (CarSim and EKF estimation)

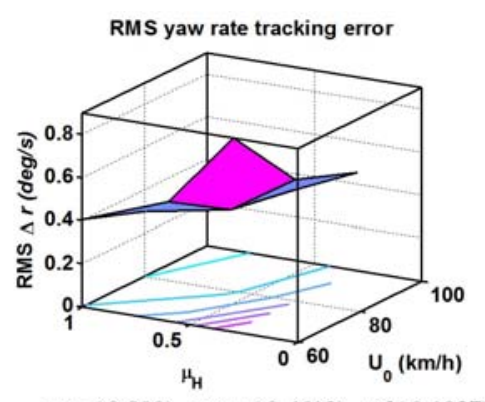

$\max (0.900), \operatorname{mean}(0.4612), \operatorname{std}(0.1937)$

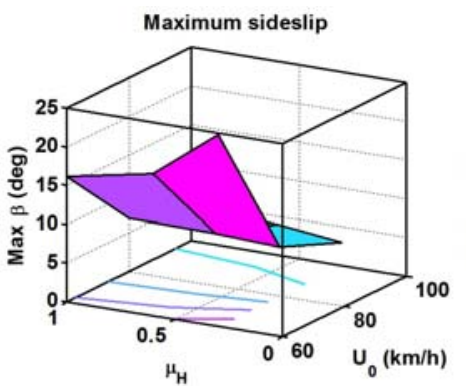

$\max (24.8)$, mean(10.1), std( 7.7)

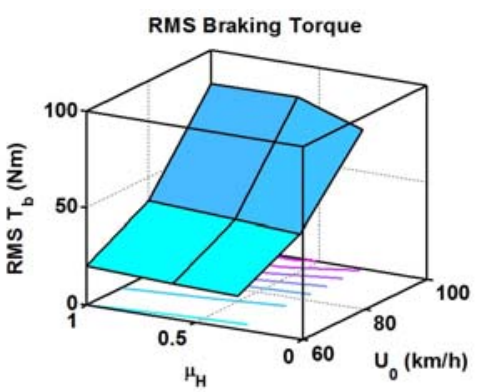

$\max (83.0)$, mean (44.4), std( 27.3)

(b) Model-plant mismatch for road surface friction (CarSim and EKF estimation)

Fig. 5.19 Performance metrics of (a) exact known road surface friction for the controller and (b) model-plant mismatch (Controller: $\mu_{H}=0.9$, Plant: various $\mu_{H}$ ) during Sine-

With-Dwell maneuver

The road surface friction model-plant mismatch assumes a controller design based on a high road surface friction (0.9) for all simulation cases, even though the actual road surface friction varies from 0.3 to 1.0 . In particular, the performance evaluation is achieved using the Sine-With-Dwell maneuver consisting of 9 different driving conditions including 3 different road surface frictions $(0.3,0.6,1.0)$ and 3 different vehicle initial speeds $(60 \mathrm{~km} / \mathrm{h}, 80 \mathrm{~km} / \mathrm{h}, 100 \mathrm{~km} / \mathrm{h})$.

Fig. 5.19 shows that under low road surface friction the performance is more degraded than in high road surface friction cases, compared to the known road surface friction case. This degradation is due to the overestimation of the reference vehicle motions based on the feedback states. Overestimation leads to low control input which is insufficient to control vehicle motions. 


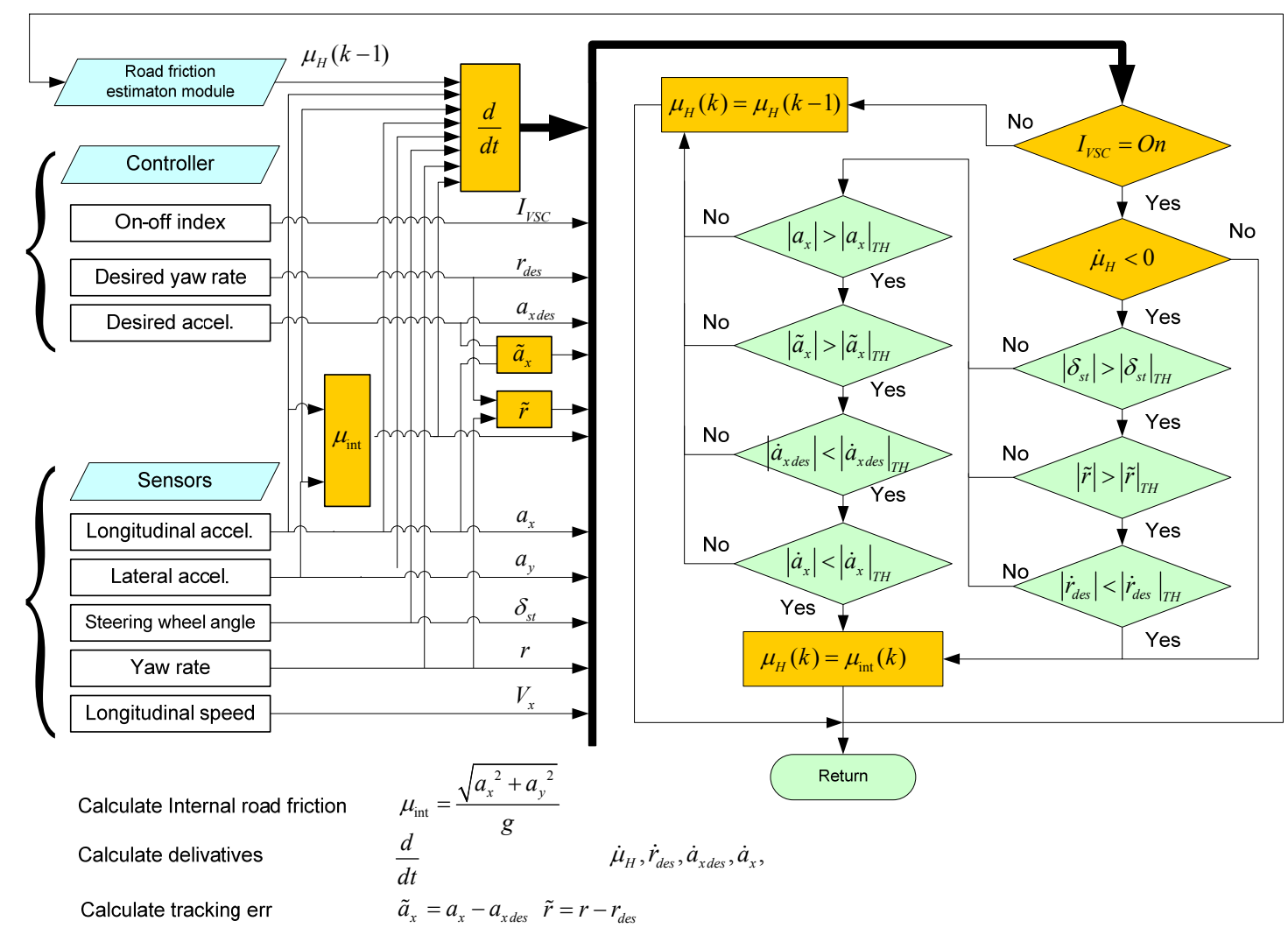

Fig. 5.20 Flow chart of road surface friction estimation algorithm

\section{(ii) VSC performance with road surface friction adaptation}

In this section, a road surface friction estimation is designed based on a simple logic that uses longitudinal and lateral accelerations by following the design concept presented in (Eckert, 1998).

$$
\mu=\frac{\sqrt{a_{x}^{2}+a_{y}{ }^{2}}}{g}
$$

The resultant acceleration of the longitudinal and lateral accelerations can represent a pick-value under a limit driving maneuver. The road surface friction estimation algorithm in Fig. 5.20 allows the estimated road surface friction to be greater than the nominal road surface friction which starts with a high road surface friction (ex. $\left.\mu_{H}=1\right)$ and updates the nominal road surface friction using Eq. (5.9) only when the 
driving maneuver belongs to limit maneuvers. In other words, the algorithm does not update the estimated road surface friction when a driving maneuver is not severe because the controller can handle the tracking of desired vehicle motions. The detailed algorithm of the road surface friction estimation is as follows:

\section{Data initialization:}

Minimum steering input rate: $|\dot{\delta}|_{\min }$,

Maximum counting number for the case $I_{V S C}=0: N_{\mu M a x}$

\section{Step 0:}

Set up initial nominal road surface friction $\hat{\mu}_{H}=1$, updating count number $N_{\mu}=0$, controller activation index at previous sample time Index $_{V S C_{-} \text {old }}=0$

\section{Step 1:}

(a) Measure accelerations $a_{x}, a_{y}$, yaw rate, and steering input $\delta$

(b) Set a controller activation index $\operatorname{Index}_{V S C}$, i.e., ( Index $_{V S C}=0$ : controller off and Index $_{V S C}=1$ : controller on) and update the counting number $N_{\mu}$

If Index VSC_old $_{1}=1 \&$ Index $_{V S C}=1, \quad N_{\mu}=0$

If Index $_{V S C_{-} \text {old }}=0 \&$ Index $_{V S C}=1, \quad N_{\mu}=1$

If Index $_{V S C_{-} \text {old }}=0 \&$ Index $_{V S C}=0, \quad N_{\mu}=N_{\mu}+1$

(c) estimated the internal road surface friction $\mu_{\text {int }}=\sqrt{a_{x}{ }^{2}+a_{y}{ }^{2}} / g$

\section{Step 2:}

(a) If $I_{V S C}=1$ or $I_{\mu} \leq I_{\mu M a x}$, go to Step 2(b). Otherwise, go to Step 3 .

(b) Calculate the derivatives of internal road surface friction, desired yaw rate, desired acceleration, and measured acceleration (ex. $\dot{\mu}_{\text {int }}=\frac{\mu_{\text {int }}-\hat{\mu}_{H}}{T_{S}}$ ) to detect vehicle motion saturation.

(c) If $\dot{\mu}_{\text {int }}<0$ and $|\dot{r}|>|\dot{r}|_{\min }$, go to Step 3 .

(d) Otherwise, update the road surface friction $\hat{\mu}_{H}=\mu_{\text {int }}$.

\section{Step 3:}

Reset $\delta_{\text {old }}=\delta$ and Index $V_{V S C \_o l d}=$ Index $V_{V S C}$ and go back to Step 1. 

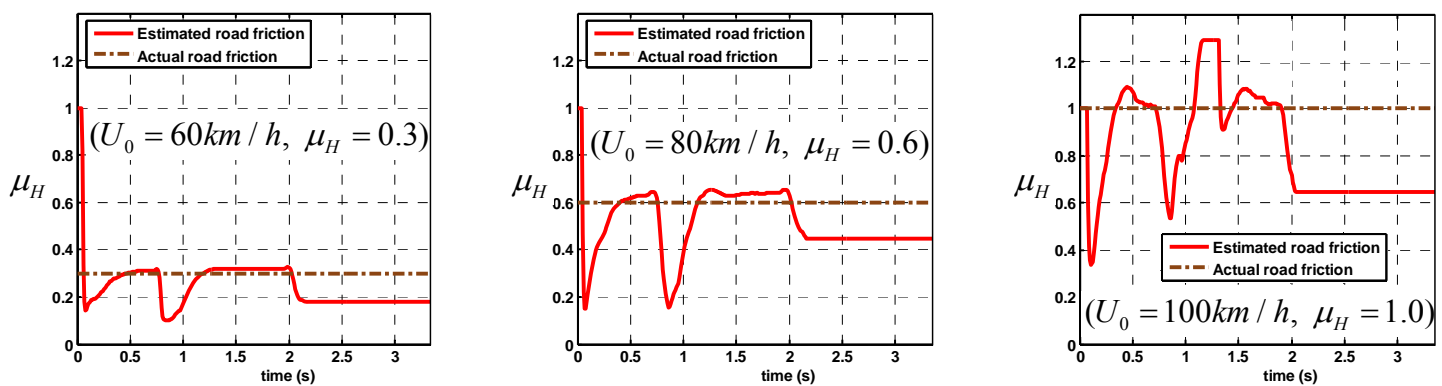

Fig. 5.21 Examples of road surface friction estimation during Sine-With-Dwell maneuver under three different driving conditions (CarSim and EKF estimation)

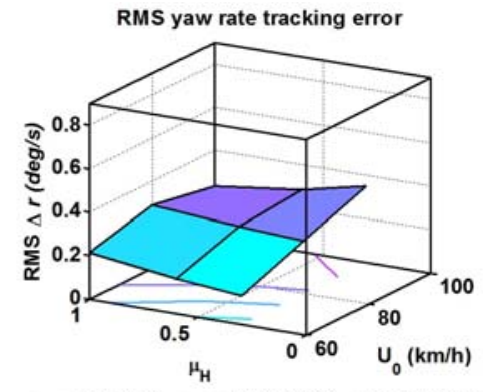

$\max (0.351), \operatorname{mean}(0.2408), \operatorname{std}(0.0671)$

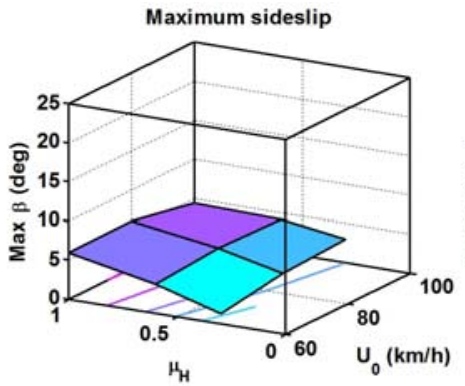

$\max (6.0), \operatorname{mean}(3.9), \operatorname{std}(1.5)$

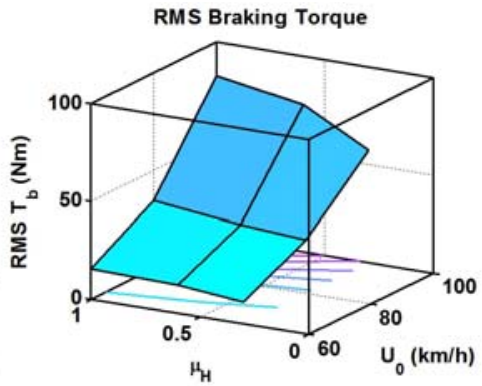

$\max (82.9)$, mean( 38.7), std( 26.8)

Fig. 5.22 Performance metrics of the road surface friction adaptation using the fixedweight LTV MPC during Sine-With-Dwell maneuver (CarSim and EKF estimation)

\section{(iii) Road surface friction estimation:}

Road surface friction estimation is performed using a CarSim vehicle model. The controller uses the estimated values using EKF given in Section 5.3.3 with the same noise condition on the measurement. This assumes the acceleration measurements are free of extraneous noise or coupling of body roll to acceleration bias - we assume that suitable filtering and compensation has been carried out.

In Fig. 5.21, the road surface friction adaptation algorithm shows that the estimated value is only close to the true road surface friction when the vehicle motion corresponds to peak transient motions. In other words, when the vehicle motions are not so severe as to lead to maximum acceleration, the road surface friction tends to be a somewhat lower estimated value than the actual value, even though the controller is activated because the logic is based on the measured combined longitudinal and lateral 
accelerations. The estimated road fiction is updated and sometimes held during transient motion in Fig. 5.21.

As shown in Fig. 5.22, the controller with the road surface friction adaptation can control vehicle motions similar to the exact model-plant match case in Fig. 5.19 (a). This controller also addressed the undesirable vehicle motions compared to the controller without road surface friction adaptation.

\subsection{MORE COMPLEX DRIVING MANEUVERS}

This section aims to demonstrate that the FICC can be employed for more complex maneuvers by tracking an additional longitudinal motion tracking objective as well as the lateral and yaw motion control which can be found in the conventional vehicle stability control. This situation is realized by an open-loop brake-in-turn maneuver under various road surface friction conditions and a closed-loop lane change maneuver under low road surface friction where a driver is supposed to apply full braking during both maneuvers. In particular, the brake-in-turn maneuver causes the left and right-hand side braking forces to be unbalanced due to a load transfer which then triggers undesirable yaw motion.

In this study, the vehicle responses of the FICC are compared to those of the pure braking control using a simply designed ABS to maximize braking force by tracking predefined peak slip ratio, even though commercial ABS controllers include the functionality with more sophisticated rules to compensate for undesirable yaw motions resulting from the unbalance right and left braking forces. (Bosch, 2004)

\subsubsection{OPEN-LOOP BRAKE-IN-TURN MANEUVER}

First, the controller is tested for the open-loop step-steer maneuver during brakein-turn under 9 different driving conditions- 3 different road surface friction $(0.3,0.6$, 

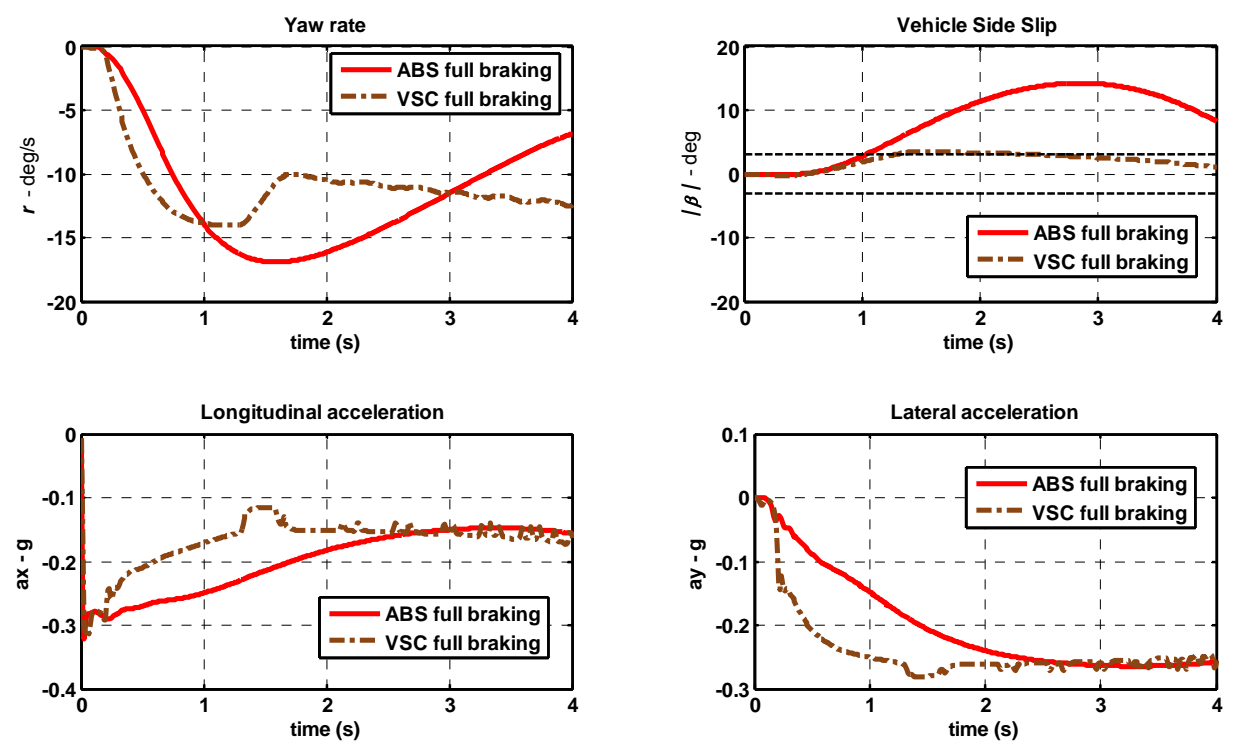

Fig. 5.23 Vehicle responses during the open-loop brake-in-turn maneuver (CarSim and EKF estimation. $U_{0}=60 \mathrm{~km} / \mathrm{h}, \mu_{H}=0.3, \delta_{H W}=180 \mathrm{deg}$ )

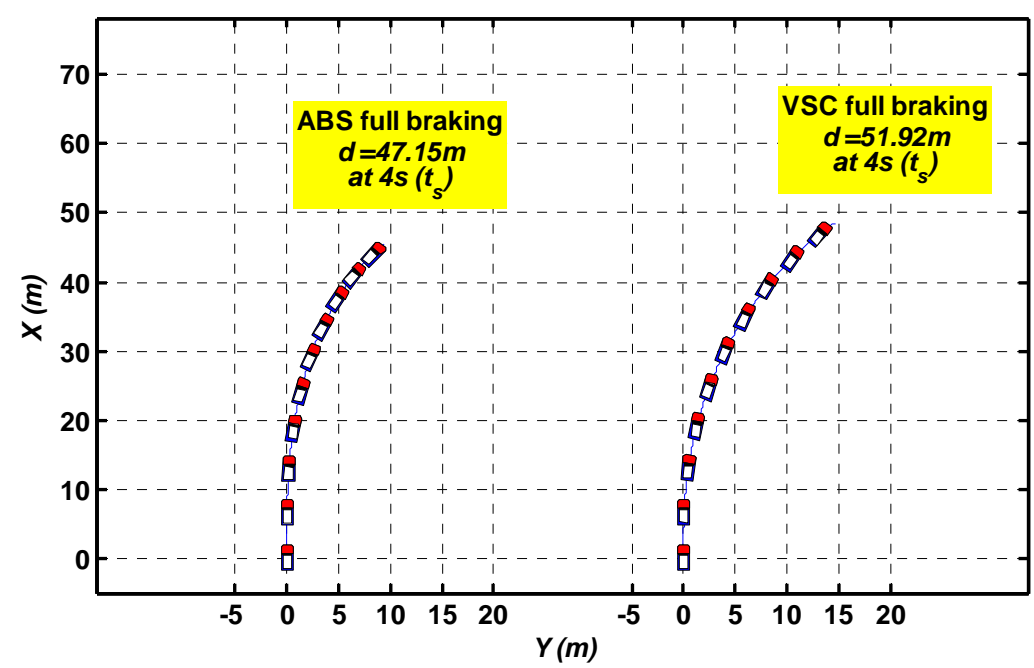

Fig. 5.24 Vehicle trajectories during the open-loop brake-in-turn maneuver (CarSim and EKF estimation. $U_{0}=60 \mathrm{~km} / \mathrm{h}, \mu_{H}=0.3, \delta_{H W}=180 \mathrm{deg}$ )

$1.0)$ and 3 different initial speeds $(60 \mathrm{~km} / \mathrm{h}, 80 \mathrm{~km} / \mathrm{h}, 100 \mathrm{~km} / \mathrm{h})$ with the step steer handwheel angle 180 degrees.

Figs. 5.23 and 5.24 show a direct comparison of two controllers in terms of vehicle responses and trajectories including moving distance for 4 seconds under low 


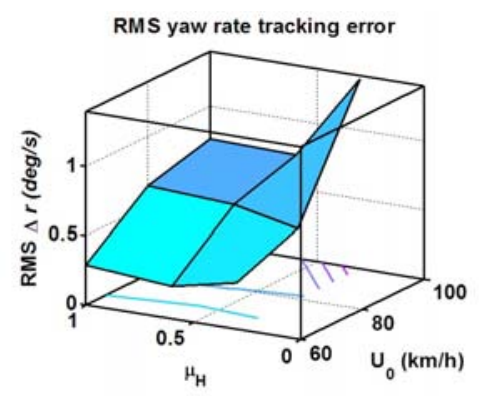

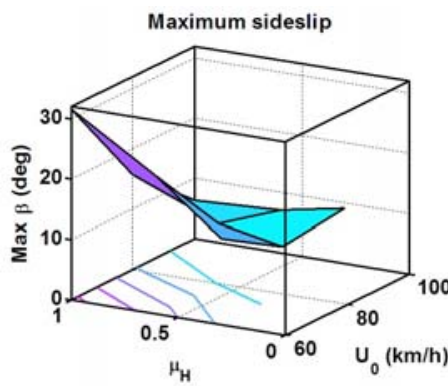

$\max (31.7), \operatorname{mean}(13.8), \operatorname{std}(8.5)$

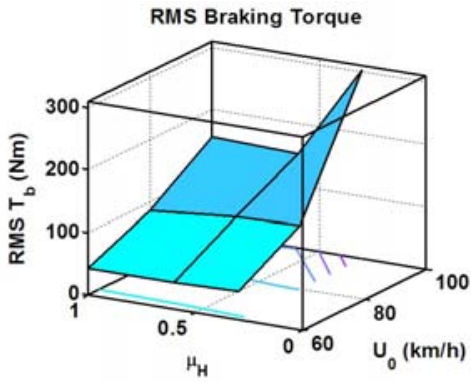

$\max (303.2), \operatorname{mean}(114.0), \operatorname{std}(83.0)$

$\max (1.379), \operatorname{mean}(0.6116), \operatorname{std}(0.3465)$

Fig. 5.25 Performance metrics of the ABS controller during an open-loop brake-in-turn maneuver $\delta_{H W}=180 \mathrm{deg}$ (CarSim and EKF estimation)

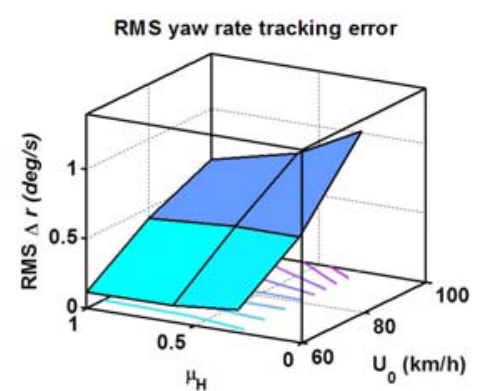

$\max (1.018)$, mean $(0.4671), \operatorname{std}(0.3090)$

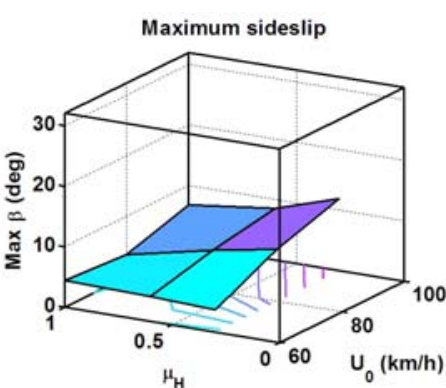

$\max (11.8), \operatorname{mean}(6.4), \operatorname{std}(2.8)$

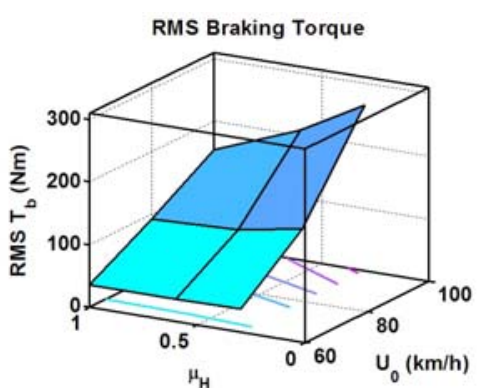

$\max (264.9)$, mean(115.7), std( 80.9)

Fig. 5.26 Performance metrics of the FICC controller during an open-loop brake-in-turn maneuver $\delta_{H W}=180 \mathrm{deg}$ (CarSim and EKF estimation)

road surface friction. As shown in Fig. 5.23, there is a balance between longitudinal and lateral acceleration clear from 0 second to 2 second to prevent sideslip increase. Moreover, Fig. 5.24 shows that the large sideslip of the ABC controller leads to a large cornering radius due to drift-out. However, the proposed VSC achieves smaller cornering radius, but sacrifices the longitudinal deceleration in terms of moving distance, $\operatorname{VSC}(d=51.92)$ and ABS $(d=47.15)$.

Fig. 5.25 shows the vehicle performance in terms of RMS yaw rate tracking error, maximum sideslip and RMS braking torque. The pure ABS controller shows the drift-out phenomena with a large sideslip angle particularly under low road surface friction. However, as shown in Fig. 5.26, the FICC controller can maintain vehicle stability for 


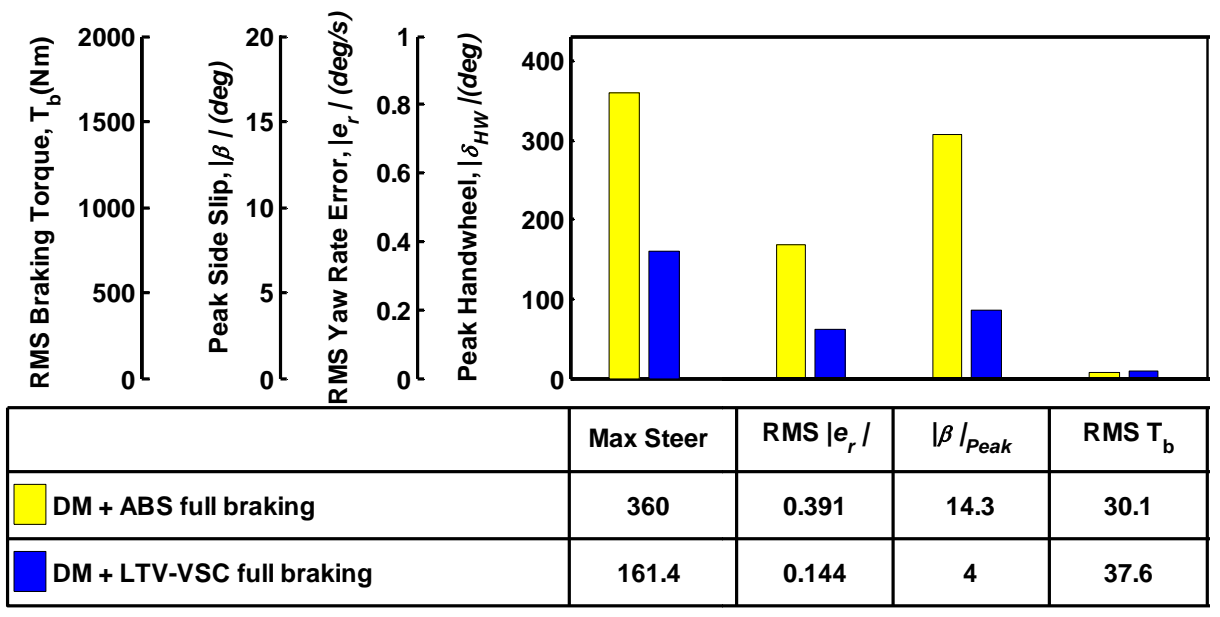

Fig. 5.27 Performance comparison during the closed-loop lane change maneuver with an ideal driver (CarSim and EKF estimation, $U_{0}=60 \mathrm{~km} / \mathrm{h}, \mu_{H}=0.3$ )

(a) Vehicle trajectories

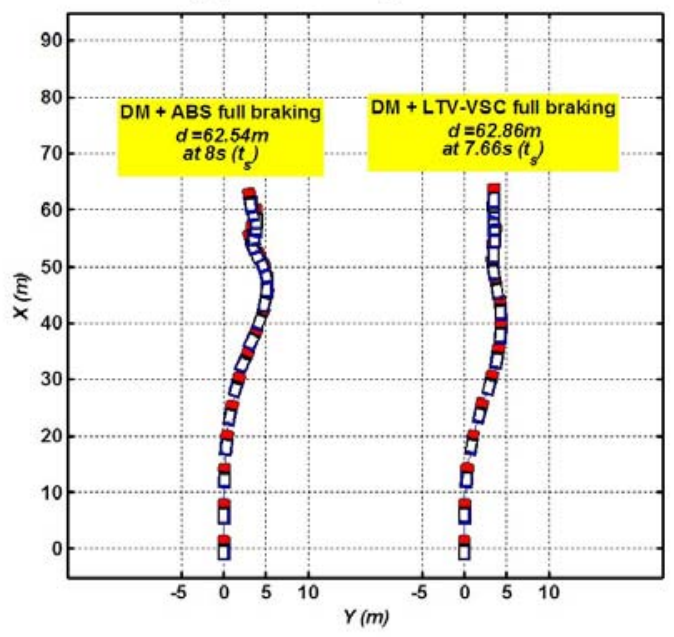

(b) Handwheel steer : DM + ABS full braking

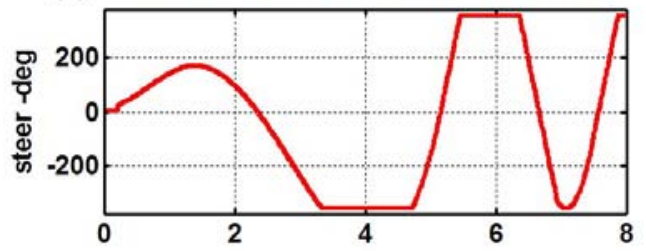

(c) Handwheel steer : DM + LTV-VSC full braking

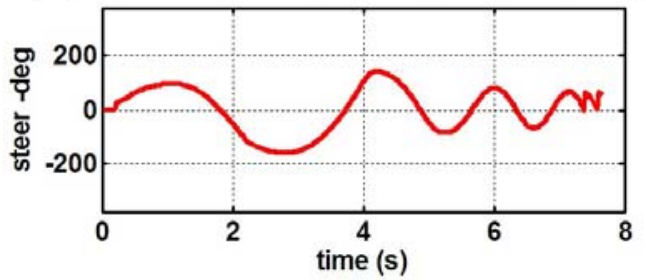

Fig. 5.28 Vehicle trajectories and handwheel steer inputs during the closed-loop lane change maneuver with an ideal driver (CarSim and EKF estimation.

$$
U_{0}=60 \mathrm{~km} / \mathrm{h}, \mu_{H}=0.3 \text { ) }
$$

overall driving conditions by preventing sideslip increase and sacrificing the longitudinal deceleration.

\subsubsection{CLOSED-LOOP LANE CHANGE MANEUVER}

For the lane change maneuver with full braking, the close-loop steer maneuver is performed with using the MacAdam driver model built in the CarSim. The MacAdam 
driver model is an optimal preview driver model designed to minimize the deviation between a target trajectory and the predicted trajectory using a linear vehicle model up to the preview time. The characteristics of the MacAdam driver model can be specified using the preview time and delay time. In this study, the preview time and the delay time are 1.1 second and 0.2 seconds respectively (Macadam, 2003). The handwheel steer and handwheel steering rate are limited to 360 degrees and 1200degree/s, respectively.

Fig. 5.27 shows the performance comparison (maximum handwheel steer, RMS yaw rate tracking error, peak sideslip and RMS braking torque) of the ABS controller and the FICC controller during the lane change maneuver with an ideal driver (driver model, DM) under the low road surface friction. The benefits of the FICC are less max handwheel input, smaller yaw rate tracking error and sideslip, compared to the ABS controller with the driver model. In Fig. 5.28, the vehicle trajectory of the ABS controller shows greater lateral displacement and large driver steering input when the vehicle moved into the second lane around the $X$-coordinate $45 \mathrm{~m}$. This seems to cause a loss of steerability due to spin-out. This spin-out leads to a driver counter steer too severe to reach the handwheel steer limit.

\subsection{ACTUATOR FAILURE MITIGATION}

In this section, actuator failure mitigation is investigated using the integrated chassis control to show the flexibility of the FICC to handle various vehicle conditions. The FICC is designed with additional active front steering control along with the individual controllable braking control at four wheels. The reason for this additional steering control is to enhance handling responsiveness by directly steering vehicle motions, especially, during braking failure situations.

Braking system failure can occur under ordinary driving conditions due to defective parts, severely worn parts, degraded parts or mechanical damaged parts (i.e. a 


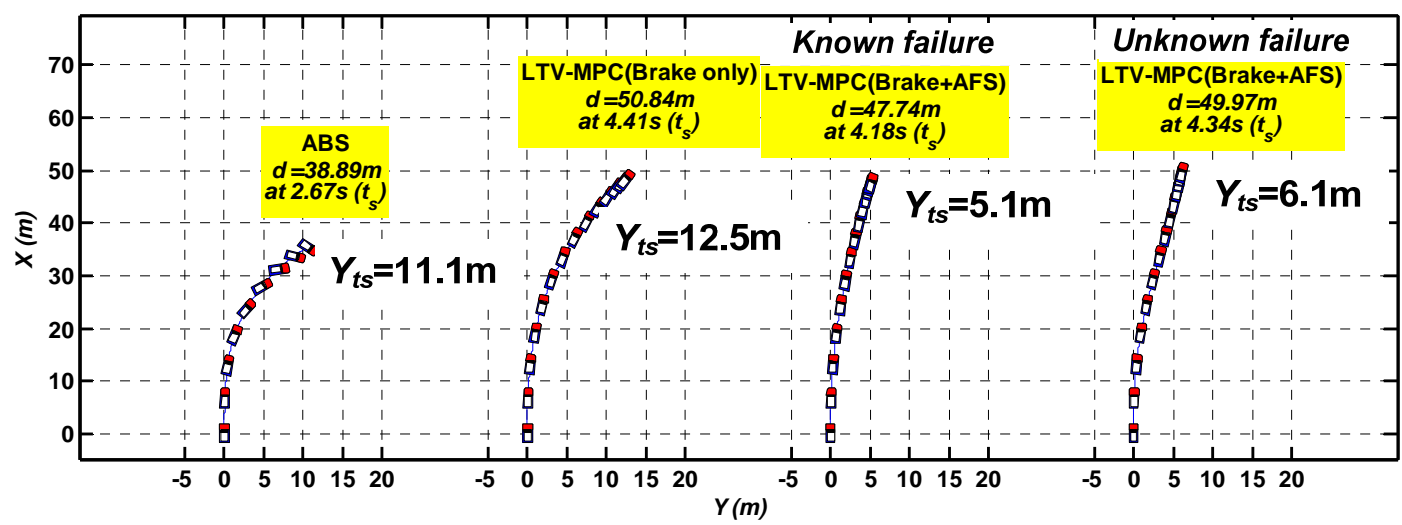

Fig. 5.29 Vehicle trajectory during open-loop straight maneuver with full braking and front left wheel braking failure (CarSim)

: Moving distance $(d)$ and lateral displacement $\left(Y_{t s}\right)$ at the stopping position

bleeder valve fracture or brake hose leakage caused by road debris) (Limpert, 1999). Some of these causes of brake failure can be detected by an ABS module that includes an electrical signal check such as a short or operational range of a circuit, wiring harness, pressure sensor, wheel speed sensor or other electric components. A model-based fault detection for a brake actuator can be found in (Rajamani et al., 2001) and (Song et al., 2003). With respect to the fault tolerant control for brake failure with the VSC, Hac et al (Hac, 2006; Hac and Dickinson, 2006) presented two approaches: rule-based control and optimal-based coordinated control. They considered the Brake- and Steer-by-Wire Systems and assumed known faults where Fault Detection Isolation (FDI) was available and no braking force was generated at the failed actuator. The rule-based control applied different rules depending on the amount of braking force loss due to actuator failure. On the other hand, the coordinated control was designed by optimization techniques using linear programming for dynamic inversion with inequality constraints.

In this study, brake failure is assumed to be detectable by the FDI technique, which can be found in brake health monitor systems of conventional ABS systems. Further, no braking force is assumed at the wheel with brake failure, for simplicity. Four different control scenarios are investigated under the assumption of front left wheel brake 
failure: (1) a simple ABS control without yaw moment compensation, (2) brake-based LTV-MPC with known failure information, (3) integrated LTV MPC using brake and AFS with known failure information, and (4) integrated LTV MPC using brake and AFS with unknown failure information. When failure information is known, the MPC controller treats the failure as a constraint. When failure information is unknown, the MPC controller calculates its control output based on nominal constraints such as wheel slip ratio limit. The simulation is performed using CarSim vehicle model for the openloop straight lane driving with full braking on a dry road $\left(\mu_{s}=0.9\right)$ with an initial speed $80 \mathrm{~km} / \mathrm{h}$. The MPC design parameters of the integrated control are given in Table E.2.

Fig. 5.29 shows the vehicle trajectory until the vehicle stops at $t_{s}$ after braking, except for the ABS case where $t_{s}$ represents the time at which the vehicle spins out with a sideslip angle of 90 degrees. All LTV-MPC controllers enable the vehicle to stop without spin-out. In particular, the integrated LTV-MPC controller with known failure information shows the best performance in terms of the small deviation from the straight lane and short stopping moving distance. The integrated controller without failure information shows better performance than the ABS controller, but worse performance than the integrated LTV-MPC controller with known failure information.

Fig. 5.30 shows vehicle responses in terms of yaw rate, side slip, longitudinal and lateral accelerations for four different control scenarios. As can be seen in Fig. 5.30, the ABS control causes the vehicle to spin out due to brake failure; however, all LTV-MPC controllers maintain vehicle stability in terms of the yaw and sideslip responses.

The counter steer of the integrated controllers is presented in Fig. 5.31. As the vehicle tends to turn to the left-hand side at the beginning, the counter-steer to the righthand side is applied by the AFS to correct vehicle motion. When the failure information is not available, the magnitude of the active front steer is larger than that resulting from the known failure information. This seems to occur because of an unbalance in braking force. This unbalance occurs in response to inappropriate control commands by the 

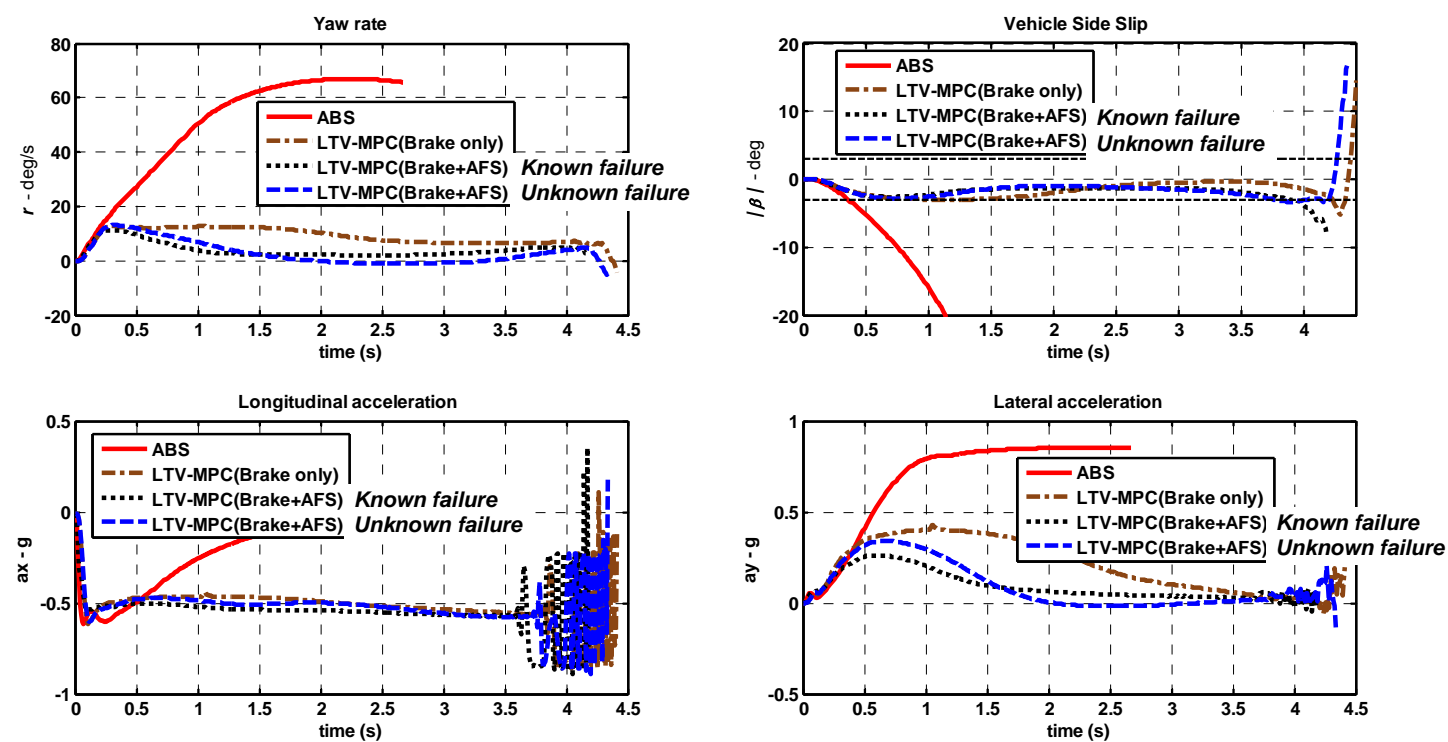

Fig. 5.30 Vehicle response comparisons during open-loop straight maneuver with full braking and front left wheel braking failure (CarSim)

Known failure

Handwheel steer : LTV-MPC(Brake+AFS)

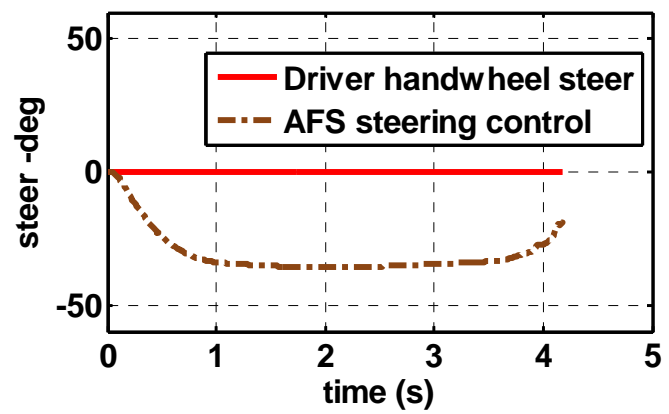

Unknown failure

Handwheel steer : LTV-MPC(Brake+AFS)

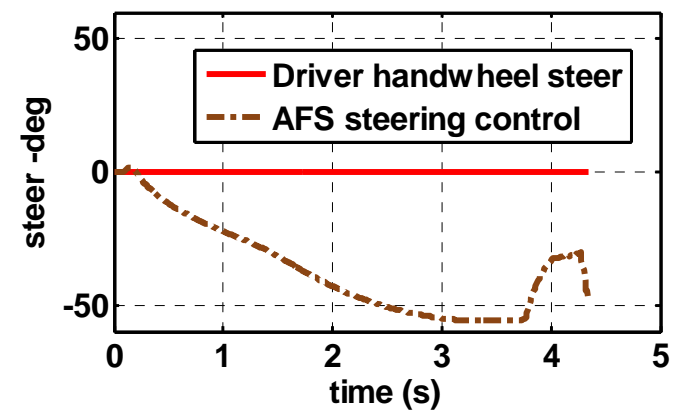

Fig. 5.31 Handwheel steer response during open-loop straight maneuver with full braking and front left wheel braking failure (CarSim)

MPC, which relies on braking apportionment at all wheels, even though one of wheels is not available. Thus, this unbalanced braking force leads to a larger steering control to compensate for the undesirable yaw motion.

\subsection{FEASIBILITY OF REAL-TIME IMPLEMENTATION}

\subsubsection{PC-BASED REAL-TIME SIMULATON}




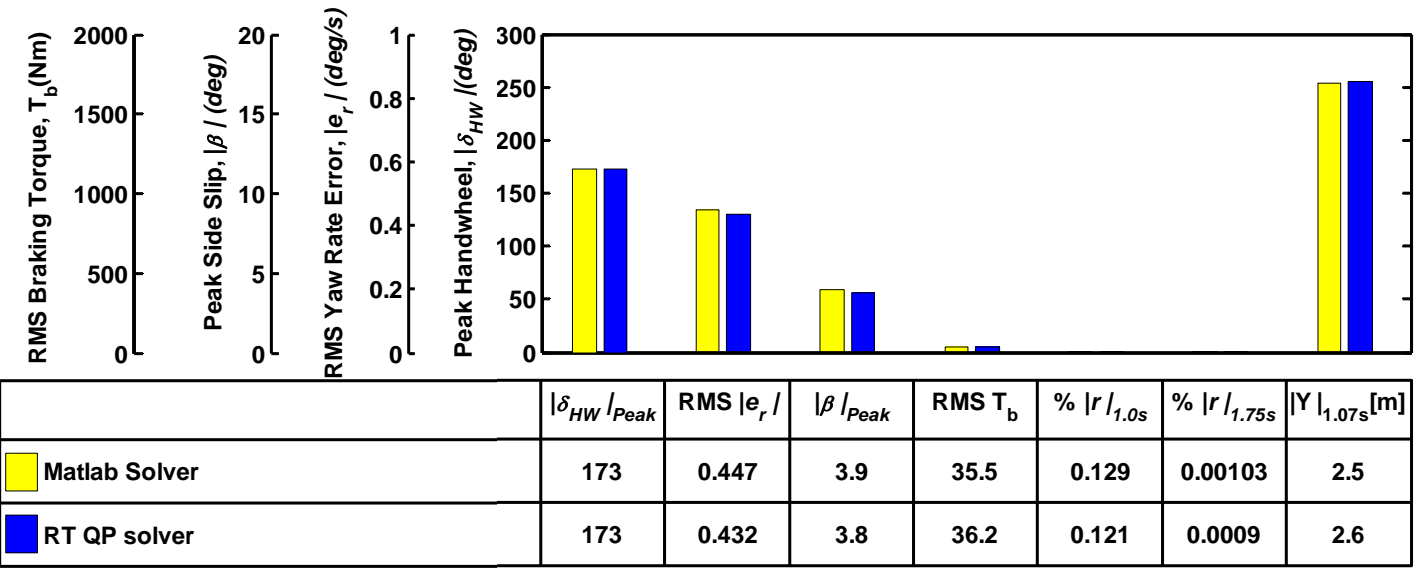

Fig. 5.32 Performance comparison of the VSC controller using real-time QP solver and Matlab QP solver during Sine-With-Dwell maneuver (8 DoF)
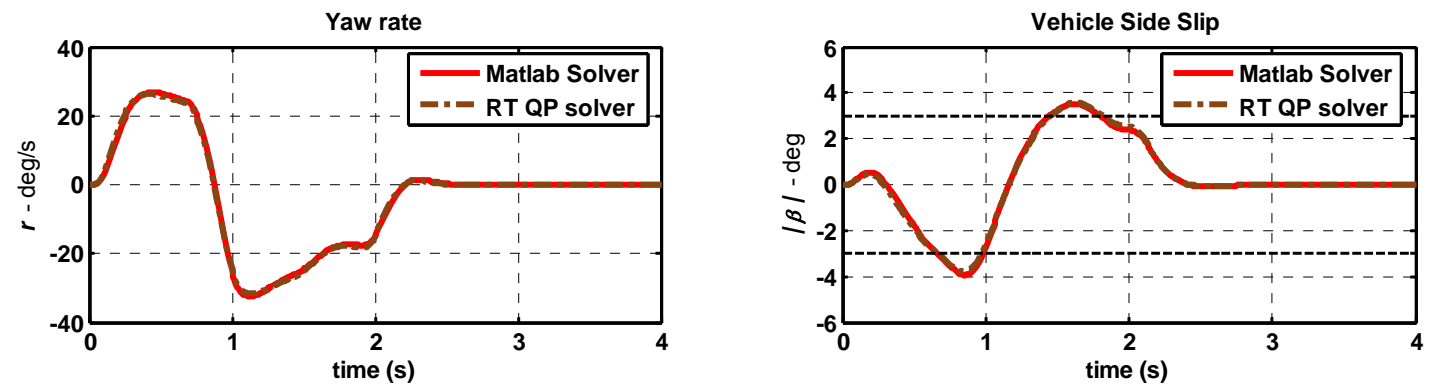

Fig. 5.33 Comparison of vehicle yaw rate and sideslip responses between real-time QP solver and Matlab solver during Sine-With-Dwell maneuver (8 DoF)

The feasibility of real-time implementation is demonstrated using Matlab toolbox Real-Time Windows Target, which includes C Mex S-function block in Simulink, on a Pentium PC platform (Intel Centrino 1.6GHz Processor). The Real-Time Windows Target provides a PC solution, Software-in-the-loop simulation, for the design and testing of real-time systems similar to Hardware-in-the-loop simulation (HILS) except that it uses a single PC as a host and target, while HILS consists of actual hardware and a computer that provide virtual plant or controller. The various application of the HILS in chassis control systems can be found in (Sorniotti, 2004; Park et al., 2005; Schuette and Waeltermann, 2005; Sorniotti et al., 2006) 
The LTV-MPC approach is one possible approach to real-time implementation in terms of a fast and reliable solver that is based on convex optimization, a subfield of mathematical optimization. The LTV-MPC properties can be described as follows: (a) the optimal solution is a global minimum when a local minimum exists, (b) the global minimum solution is convex, and (c) there exists at most one minimum if the optimization problem function is strictly convex.

In this study, the real-time quadratic (RT-QP) solver used in C Mex S-function is FSQP (Feasible Sequential Quadratic Programming), which can solve nonlinear constrained optimization as well as quadratic linear constrained optimization. FSQP was originally developed by Andre Tits' research group at the Institute for Systems Research (ISR), University of Maryland, College Park. Two versions of FSQP are available CFSQP Version 2.5d (C version) and FFSQP Version 3.7b (Fortran version). The detailed procedures of the PC-based real-time simulation is provided in Appendix D.

Using Matlab toolbox Real-Time Windows Target, the proposed vehicle stability controller was successfully performed in Software-in-the-loop simulation as real-time development environments for the typical MPC design parameters of this thesis, i.e., sample time $T_{s}=0.002 s, H_{P}=10$, and $H_{U}=1$. In the next section, more quantitative approaches are presented to investigate the effect of control design parameter to the realtime controller development in terms of computational loads.

\subsubsection{EVALUATION OF REAL-TIME SIMULATION PERFORMANCE}

\section{(i) Comparison of controller performance between the real-time QP solver and off-line QP solver}

First, this section evaluates the accuracy of the controller design using the RT solver and compares the latter to a controller based on popular commercial software, Matlab QP solver-quadprog (The MathWorks, 2006a). The difference between each VSC controller is compared by investigating controller performance. The simulation is 


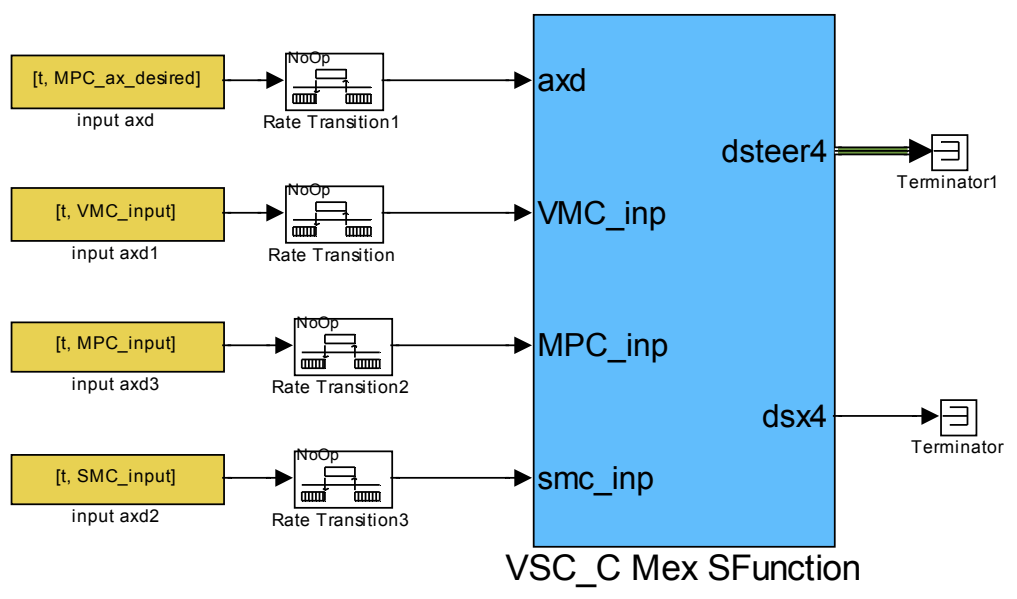

Fig. 5.34 Simulink block for the evaluation of real-time simulation performance

performed under the same simulation environment such as the brake-based LTV-MPC controller, an 8 DoF nonlinear vehicle model for a Sine-With-Dwell maneuver on a dry road with an initial speed of $80 \mathrm{~km} / \mathrm{h}$, and the same control design parameters.

As shown in Figs. 5.32 and 5.33, the vehicle responses are almost identical to each other, except that the VSC using RT-QP solver shows a little less peak yaw rate and sideslip. This difference resulting from the VSC seems to be due to the difference in the programming development environment. For example, the VSC using RT-QP solver was developed using C-programming language and the VSC using Matlab tool box QP solver was developed using Matlab/Simulink, but both QP solvers provide the same optimal solution for a simple optimization problem.

\section{(ii) Computational load of the real-time QP solver}

This section focuses on the quantitative evaluation of the feasibility of real-time implementation by running the real-time QP solver-based controller on a Pentium PC platform (Intel Centrino 1.6GHz Processor). The feasibility of real-time implementation is measured by the mean value of real-time ratio, which is defined by the computational time on the PC platform versus nominal simulation time on Matlab/Simulink environment. This mean value of real-time ratio represents the average computational 
load during the controller operation. As shown in Fig. 5.34, the Simulink block is simplified by loading vehicle information which is used for the controller instead of using a vehicle model. Unlike the mean value of real-time ratio, the peak value of real-time ratio defined based on each instant, although the entire simulation time can be used directly to assess the feasibility of the real-time implementation. In other words, when the peak real-time ratio is less than one, this mean that the direct implementation of the control signal from the PC to real systems is theoretically possible. However, an appropriate interface between the PC and real systems is necessary and this can be achieved with additional interfacing software and hardware such as LabVIEW, dSPACE AutoBox system and Opal-RT, and can be found in HILS implementation or a real car test (Borrelli et al., 2005; Park et al., 2005; Sorniotti et al., 2006; Falcone et al., 2007a).

In this study, the computational load in terms of the mean value of real-time ratio is first evaluated for different sample times where the computational time on the PC platform is inversely proportion to the sample time by assuming that the controller performs its computation procedure once at each sample time. The reason to consider the sample time is that this is one way to reduce the computational capacity necessary to find a solution within a limited time interval. The smaller sample time (high sample rate) represents a fast controller which can perform the computation procedure many times over at each sample instance for a certain controller operation time interval. Regarding other design parameters, such as output prediction horizon, control prediction horizon and stopping criterion of the MPC solver, these factors can be critical issues for real-time implementation in large plant systems such as chemical plants (Findeisen, 2005) and nonlinear MPC problems. However, the development of efficient computational hardware in recent years helps the controller to address the computational capacity including computer memory size. Moreover, the vehicle control systems based on the convex linear quadratic optimization is more suitable for the real-time implementation than nonlinear MPC solver-based controller because an optimal solution of the convex 
(a) Mean value of real-time(RT) ratio: Ts vs. $H_{P}\left(H_{U}=1\right)$

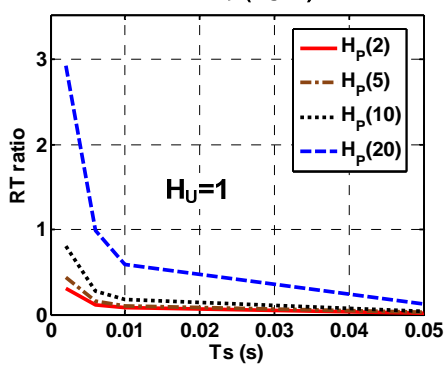

(b) Mean value of real-time(RT) ratio: $H_{P}$ vs. $H_{U}(T s=0.01 s)$

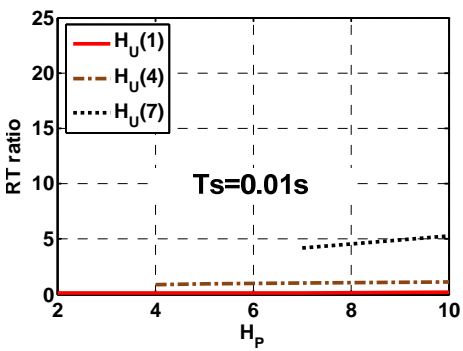

(c) Mean value of real-time(RT) ratio: $H_{P}$ vs. $H_{U}(T s=0.002 s)$

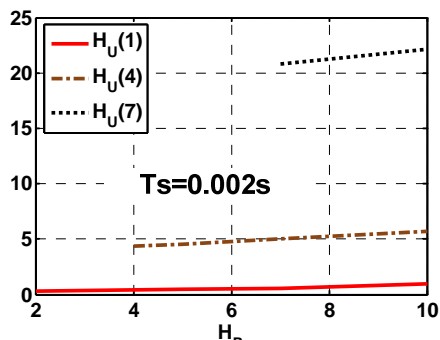

Fig. 5.35 Mean value of real-time ratio with respect to (a) sample time and $H_{P}$ (fixed $\left.H_{U}=1\right)$, (b) $H_{P}$ and $H_{U}$ of the LTV MPC-based VSC (fixed $T_{s}=0.01 s$ ), and $H_{P}$ and $H_{U}$ of the LTV MPC-based VSC (fixed $T_{s}=0.002 s$ )

linear quadratic optimization always exists, whereas the nonlinear optimization does not guarantee this.

Fig. 5.35 shows the mean value of real-time ratio with respect to sample time, output prediction horizon and control prediction horizon using the LTV MPC-based VSC controller with fixed weights of MPC cost function during the brake-in-turn step steer maneuver $\left(\delta_{H W}=180 \mathrm{deg}, U_{0}=60 \mathrm{~km} / \mathrm{h}, \mu_{H}=0.3\right.$ ). Fig. 5.35(a) shows the mean value of real-time ratio with respect to several sample times from 0.002 to 0.05 second and different prediction horizon $H_{P}$. The effect of the prediction horizon $H_{P}$ and control horizon $H_{U}$ is shown in Figs. 5.35(b) and (c) for fixed sample time 0.01s and 0.002s, respectively. The mean value real-time ratio varies inversely with respect to sample time $T_{S}$, output prediction horizon $H_{P}$, and control prediction horizon $H_{U}$. Most notable is that the effect of the control prediction horizon is more dominant than other factors.

Fig. 5.36 shows the number of iterations required for the QP solver to converge at each instant, and can be considered as a surrogate for real-time ratio when the computational load is proportional to the number of iterations the QP solver needs to find optimal solutions. This iteration number depends on particular driving conditions, for example, the iteration number during initial transient motions is higher than it is for the later vehicle motions, as shown in Fig. 5.36. The maximum iteration number of the QP solver is proportional to the control prediction horizon, however, less sensitive to the 
(a) Iteration number of QP solver $(\mathrm{Ts}=\mathbf{0 . 0 1 \mathrm { s } )}$

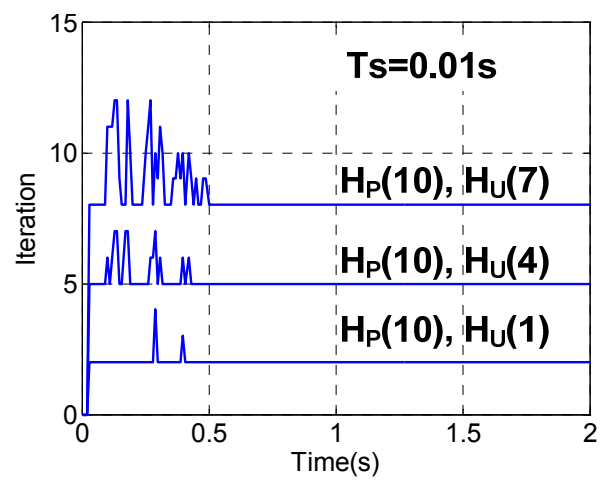

(b) Iteration number of QP solver $(\mathrm{Ts}=\mathbf{0 . 0 0 2 s})$

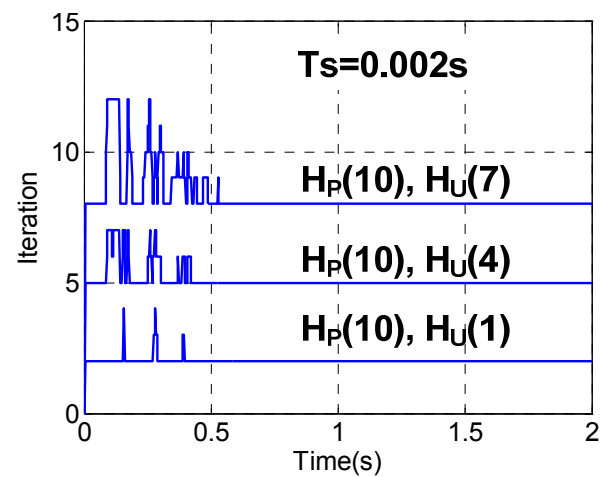

Fig. 5.36 Number of iteration for the QP solver to converge at each instant

sample time. The minimum number of QP solver iterations is exactly proportional to the control prediction horizon.

According to the quantitative evaluation in terms of real-time ratio and the number of iterations required for the QP solver, the smaller values of sample time, output prediction horizon and control prediction horizon are preferable for lower computational load; however degradation of controller performance is expected. Thus, the appropriate trade-off between controller performance and hardware cost should be considered because a high level of computer performance is usually more costly to implement.

\subsection{SUMMARY}

The benefits of VSC are first evaluated using as a guideline controller tuning that maintains vehicle sideslip at less than 5 degree from a handling stability viewpoint as first priority, and then minimizes the yaw rate tracking error from a handling responsiveness viewpoint. The off-line tuning using commercial optimization software was performed by minimizing the cost function defined as the RMS yaw rate tracking error with a sideslip constraint of $\beta(t) \leq 5 \mathrm{deg}$.

For the exact model-plant match, the proposed LTV MPC-based controller achieves stable vehicle motion more efficiently than both the rule-based reference 
controller and the LTI MPC-based controller during a Sine-With-Dwell maneuver. The LTV MPC-based controller also uses significantly less brake torque, while providing a vehicle response very similar to the rule-based controller. Regarding the rule-based controller, a more sophisticated design may improve its performance, but developing appropriate control rules for this would require considerable time and effort. By keeping overall vehicle motions similar to those of other control scenarios, the simulation results confirm the benefits of the flexible actuator apportionment as a result of updating the nonlinearity of the MPC model compared to simple reference controllers.

The robustness of the controller is investigated for various driving conditions, model-plant mismatch, actuator time constants, vehicle state and road surface friction estimation. Through numerous simulation studies, the dominant factors of the proposed control design parameters are the choice of the weights of the cost function and the reference vehicle motions which is saturated by road surface friction.

The effectiveness of the proposed controller was successfully demonstrated during more complex drive maneuvers by adding longitudinal full braking demand as well as lateral and yaw motion tracking. The flexibility of the controller in terms of actuator extension was shown to mitigate actuator failure using AFS and braking control. The feasibility study of real-time implementation was performed through the PC-based real-time simulation using Matlab toolbox Real Time Simulation Target.

The next chapter will consider an added level of functionality based on the same control structure, an autonomous control for collision avoidance systems (CAS); here the main control architecture is left intact, and reference vehicle motions are adapted in the upper layer. 


\section{CHAPTER 6}

\section{CONTROL METHODOLOGY FOR COLLISION AVOIDANCE SYSTEMS}

This chapter considers an autonomous driving functionality for the collision avoidance systems (CAS) using the FICC, as described in 3.1. The proposed control methodology includes both lateral and longitudinal control, extending the functionality of the vehicle stability controller by adapting the reference vehicle motions at the upper level of the controller. The reference vehicle motions of CAS in the upper layer are obtained by simple and real-time implementable control logic, a kinematic policy (KP) for collision avoidance. The KP determines reference yaw rate and two-dimensional mass center accelerations using simple information about range and azimuth angles for multiple points that bound the available vehicle trajectory, and prioritizes yaw motion response based on the worst case collision threat. This KP approach for CAS is more practical than trajectory tracking approaches which can be found in conventional optimal driver model or collision avoidance control because the KP does not need a pre-defined a reference path and does not need any computationally intensive optimization of the vehicle motion control. The decision or timing of driver's input overriding will not be covered. Further for simplicity road circumstance information such as road boundary and obstacle detection (Prakah-Asante et al., 2003) is assumed available.

The background of the collision avoidance systems is summarized in Section 6.1. The overall control architecture and the KP are briefly described in Section 6.2 and in Section 6.3, respectively. The controller implementation is given in Section 6.4. Then Section 6.5 presents simulation results. Finally, Section 6.6 summarizes findings and presents overall conclusions from the study. 


\subsection{REVIEW OF COLLISION AVOIDANCE SYSTEMS}

Vehicle safety devices have been developed to avoid car crashes and minimize harmful effects of accidents. These vehicle safety devices include both passive and active safety systems: the passive safety systems such as airbags or seatbelts are aimed to reduce injury after an accident, and the active safety systems such as ABS (Anti-locking Braking System) or VSC (Vehicle Stability Control) are primarily designed to prevent car accidents from occurring at first. Recently, the evolution of the vehicle safety systems are aimed to provide solutions such as collision avoidance systems (CAS) by providing warnings to the driver, or even control commands to the vehicle to avoid or minimize the harmful effects of an accident (Takahashi and Asanuma, 2000; Beutnagel-Buchner et al., 2004; Knoll, 2006; Ohue et al., 2006).

The CAS can be achieved by maximizing braking forces to minimize stopping distance or by conducting evasive maneuver to avoid an obstacle. For minimum stopping distance control, methods to obtain activation timing may be found in (Seiler et al., 1998; Zhang et al., 2006). For evasive maneuvers, path-following methods have been proposed where the target trajectory is obtained using optimization technique (Macadam, 2003; Gordon and Best, 2006; Hattori et al., 2006) or string theory (Sledge Jr. and Marshek, 1998; Hilgert et al., 2003). In (Hattori et al., 2006), a nonlinear optimization method was used to calculate optimal target trajectory and optimal chassis control was implemented to achieve the target trajectory.

The path following approach to collision avoidance is however complex and requires two stages of optimization (first define the intended path, then apply steering and/or brakes to follow that path) that may need to be repeated at high frequency in the rapidly changing conditions prior to a crash or near crash. In order to provide a driver model without a target trajectory generation, Gordon and Magnuski proposed a simple 
kinematic policy which determines reference inputs using simple information about range and azimuth angles for multiple points that bound the available vehicle trajectory (Gordon and Best, 2006). In that paper, it was shown that normal driving - lateral and longitudinal vehicle control using road boundaries to constrain the trajectory - is possible without the need to pre-define a reference path, and without need for computationally intensive optimization. Indeed, for collision avoidance applications, this reference may be obtained from commercially available radar systems, so the method appears attractive from the practical perspective.

\subsection{OVERALL CONTROLLER STRUCTURE}

Flexible Integrated Chassis Control for CAS shares the same hierarchical architecture as the VSC, consisting of three layers: (1) vehicle motion control as an upper layer, (2) an intermediate layer for the actuator apportionment using Model Predictive Control (MPC) and (3) a lower layer for individual actuator control. Compared to the upper layer design of the FICC for VSC, the FICC for CAS uses a kinematic policy instead of a driver's braking command through brake pedal and the steady state bicycle model for a reference yaw motion in the VSC to determine longitudinal and yaw reference vehicle motions respectively. More detail of the kinematic policy is presented in Section 6.3, and the details of the upper layer design are described in Chapter 3.

For actuator apportionment, the MPC of the FICC for CAS also is designed using a 3DoF nonlinear vehicle model (longitudinal, yaw and side slip) as a predictive model with Active Front Steer in addition to the brake actuators.

For simplicity, it is assumed that all vehicle variables, brake torques, tire forces and the surface friction coefficient are available to the controller by direct measurements or appropriate estimations (Ray, 1997; Bevly, 2004). 


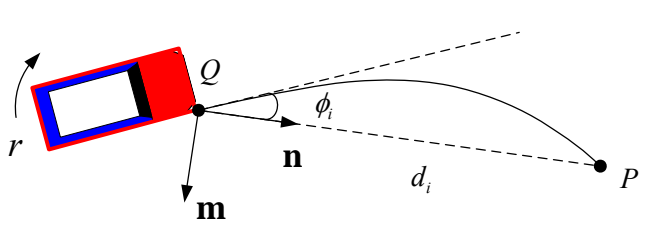

(a)

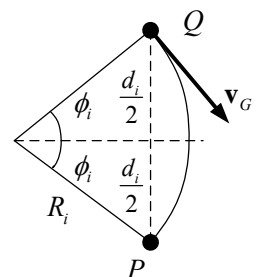

(b)

Fig. 6.1 (a) Arc-like projection of relative motion between vehicle and external reference point and (b) Simplified geometry of the projected arc

\subsection{KINEMATIC POLICY}

\subsubsection{KINEMATIC POLICY PRINCIPLE}

The vehicle motions, in the form of reference yaw rate and two-dimensional mass center accelerations, are determined using a kinematic policy for collision avoidance previously proposed in the context of a driver model (Gordon and Magnuski, 2006). The kinematic policy uses simple information about range and azimuth angles for multiple points that bound the available vehicle trajectory, and prioritizes yaw motion response based on the worst case collision threat. For simplicity, road information is supposed to be available by radar, vision systems or other devices with appropriate signal processing (Prakah-Asante et al., 2003).

In Fig. 6.1 (a), the motion of the right front corner of the vehicle $Q$ is viewed relative to the motion of a static right-hand boundary point of the road, $P$. The combination of vehicle mass center velocity $\mathbf{v}_{G}$ and vehicle yaw rate $r$, if perceived as constant, projects an arcing motion of $Q$ relative to $P$. The figure illustrates the critical case where forward motion would intersect with the boundary point. The same geometry is redrawn more simply in Fig. 6.1 (b) where $d_{i}$ is the relative distance from the front corner and $\phi_{i}$ is an azimuth angle at the $i$ th boundary point.

From this, the arc radius $R_{i}$ is found to be

$$
R_{i}=\frac{d_{i}}{2 \sin \phi_{i}}
$$


and for scalar vehicle speed $U=\left|\mathbf{v}_{G}\right|$, the critical yaw rate $\overline{r_{i}}$ is given by

$$
r<\bar{r}_{i}=\frac{2 U \sin \phi_{i}}{d_{i}}
$$

Boundary points are combined using an edge signature value

$$
\sigma_{i}=\left\{\begin{array}{lll}
+1 & \text { if } & P_{i} \in \text { Right-hand boundary points } \\
-1 & \text { if } & P_{i} \in \text { Left-hand boundary points }
\end{array}\right.
$$

This is results in a simple multi-point geometric criterion for avoidance on both boundaries simultaneously:

$$
e_{i}(t)=\sigma_{i} \cdot\left(r(t)-\overline{r_{i}}\right) \leq 0
$$

Here $r(t)$ is the current vehicle yaw rate and $\bar{r}_{i}$ is given in Eq. (6-2). Yaw velocity correction is then based on the star point - if any reference point conflicts with condition in Eq. (6.4), $i^{*}$ denotes the 'worst offender':

$$
i^{*} \equiv \arg \left(\max _{i} e_{i}\right)
$$

and the yaw velocity reference is given by the corresponding yaw rate

$$
r_{\text {ref }}=\bar{r}_{i}
$$

If on the other hand condition in Eq. (6.4) is satisfied by all points within the horizon, no steering correction is assumed to be required, and this is implemented via

$$
r_{r e f}=r(t)
$$

For a normal driving speed control as a simple autonomous driver model, the normal driving target speed is defined in terms of a conservative deceleration requirement to the horizon point in (Gordon and Magnuski, 2006):

$$
U_{r e f}=\sqrt{2 a_{r e f} d_{H}}
$$

where $d_{H}(t)=\left|\mathbf{x}_{H}(t)-\mathbf{x}_{D}(t)\right|$ is the instantaneous distance from the driver to the horizon point, and $a_{r e f}$ is a given fixed reference acceleration. However, unlike the normal driving speed control, the reference speed to achieve full braking is determined by a proportional control using a feed-back current acceleration as given in Chapter 3.

$$
U_{\text {ref }}=U(t)+\left(a_{\text {ref }}+K_{\text {Paccel }}\left(a_{\text {ref }}-a(t)\right)\right) \cdot T_{s} \quad \text { if } a_{\text {ref }} \in \text { full braking }
$$




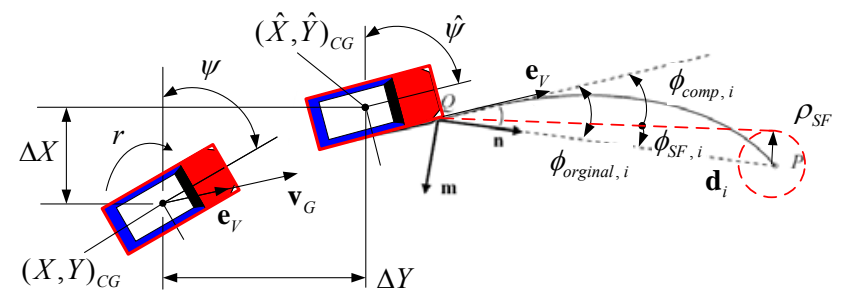

Fig. 6.2 Concepts of compensation time $\tau_{\text {comp }}$ and safety margin radius $\rho_{S F}$

\subsubsection{KP DESIGN PARAMETER}

The critical yaw rate given in Eq. (6-2) is a function of an azimuth angle and relative distance of lane boundary points. Thus the modification of the critical yaw rate change can be obtained by introducing two design parameters: a compensation time lag $\tau_{\text {comp }}$ and a safety margin radius $\rho_{S F}$, as shown in Fig. 6.2. The compensation time lag $\tau_{\text {comp }}$ is aimed to model driver anticipation used to offset actuator time delay or phase lag in a yaw motion. The safety margin radius $\rho_{S F}$ is intended to make the control a little aggressive by modifying the azimuth, i.e., decreasing the azimuth for the right-hand boundary point and increasing the azimuth of the left-hand boundary point, inversely. The modified azimuth $\phi_{c o m p, i}$ at the $i$ th boundary point is as follows:

$$
\phi_{\text {comp }, i}=\phi_{\text {orginal }, i}-\phi_{S F, i}
$$

Here the original azimuth angle and the safety margin angle are $\phi_{\text {orginal }, i}=\cos ^{-1}\left(\mathbf{e}_{V} \cdot \hat{\mathbf{d}}_{i}\right)$, $\phi_{S F, i}=\rho_{S F} / \hat{\mathbf{d}}_{i}$, respectively. $\vec{e}_{V}$ is a unit vector coinciding with a vehicle heading angle, and $\hat{d}_{i}$ represents the compensated relative distance with anticipated vehicle motions in terms of a compensated coordinate $(\hat{X}, \hat{Y})_{C G}$ and yaw angle $\hat{\psi}$, which is evaluated using current longitudinal an lateral velocities $\left(V_{x}\right.$ and $\left.V_{y}\right)$, yaw rate $r$ and the compensation time lag $\tau_{\text {comp }}$ :

$$
\begin{gathered}
\hat{X}=X+\Delta X=X+\left(V_{x} \cos \psi-V_{y} \sin \psi\right) \tau_{\text {comp }}, \hat{Y}=Y+\Delta Y=\left(V_{x} \sin \psi+V_{y} \cos \psi\right) \tau_{\text {comp }}(6.1 \\
\text { and } \hat{\psi}=\psi+r \tau_{\text {comp }}
\end{gathered}
$$


Moreover, the minimum preview distance $d_{0 \text { min }}$ is used to prevent the critical yaw rate from becoming too large when the relative distance $d_{i}$ is too small:

$$
d_{0 \min }=\max \left(5, \mid \mathbf{v}_{G} \cdot \tau_{\text {comp }}\right)
$$

The compensation time $\tau_{\text {comp }}=0.2 \mathrm{sec}$ and the safety margin radius is $\rho_{S F}=0.5 \mathrm{~m}$ except the corner point ' $P_{c}$ ', which is assumed as a known position, as show in Fig. 6.4. At the corner point, the adaptive safety margin radius is used as follows.

$$
\rho_{S F \text { corner }}(t)=\rho_{S F}+K_{S F R}\left(d_{\text {corner }}(t)-d_{0 \text { min }}\right)
$$

Here $K_{S F R}$ is constant $(0.18), d_{\text {corner }}(t)$ is the relative distance between the vehicle and the corner point of the boundary, and $d_{0 \text { min }}$ is given in Eq. (6.12). The purpose of Eq. (6.13) is to generate more aggressive steering input by increasing its azimuth angle to move fast toward the target lane when the distance of the corner point is large.

Regarding the reference speed control, the reference acceleration and the proportional gain in Eq. (6.13) are $a_{\text {ref }}=-1 \cdot g$ and $K_{\text {Paccel }}=3$ respectively where $g$ is a gravitational acceleration $9.8 \mathrm{~m} / \mathrm{s}^{2}, a(t)$ is a current resultant acceleration of longitudinal and lateral accelerations, and $T_{s}$ is a sample time. Then the reference speed $U_{r e f}$ is used as a reference longitudinal velocity for the intermediate layer under the assumption of relatively small lateral velocity.

\subsection{CONTROLLER IMPLEMENTATION}

The proposed controller was investigated under an emergency lane change maneuver with full braking where vehicle motions correspond to severe transient maneuvers. Simulations were performed in the CarSim (Mechanical Simulation, 2004) and Matlab/Simulink simulation environment(The MathWorks, 2005). A standard 'big sedan' model in the CarSim is used as well as the MacAdam driver model built in the CarSim used to run a closed-loop steering maneuver as an idealized driver that may be compared with the proposed controller. The characteristics of the MacAdam driver 

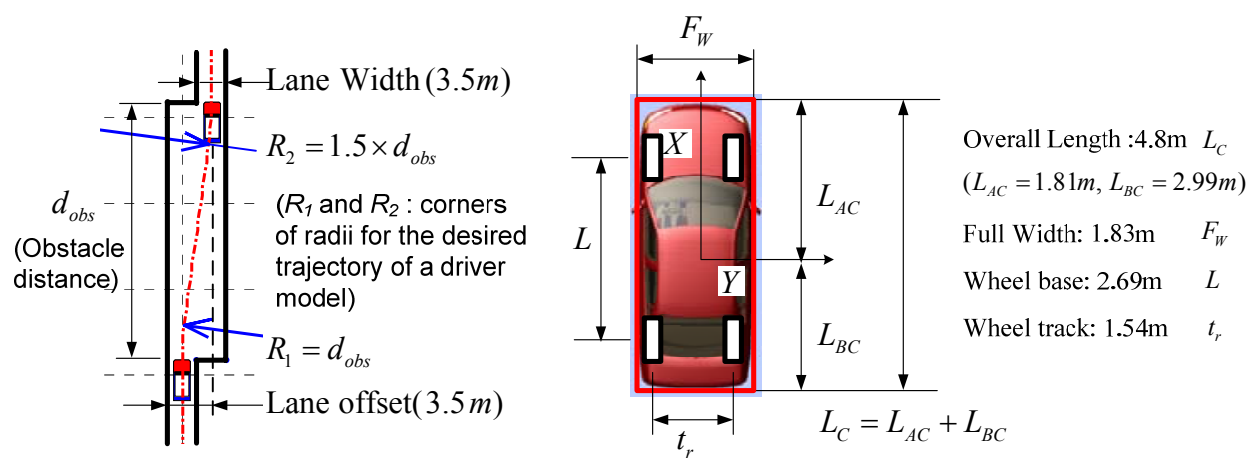

Fig. 6.3 Course layout for emergency lane change and vehicle outline

model is specified using the preview time 1.1 second and delay time 0.2 seconds, respectively (Macadam, 2003). The CarSim plant model is simulated with a fixed time step 0.002 s on a dry road $\left(\mu_{H}=0.89\right)$.

\subsubsection{COURSE LAYOUT AND VEHICLE OUTLINE}

Fig. 6.3 shows the course layout for emergency lane change maneuver as well as the basic vehicle outline. The center line of the road is used as a normal target trajectory for the driver model and its curvature is smoothed by corners of radii $R_{1}$ and $R_{2}$. We should mention that this trajectory is very important for the driver model performance but the trajectory used in this paper seems to be suitably simple and effective for the relative comparison with the proposed controller. The geometry data of the vehicle outline corresponds to a mid-size passenger car. The clearance between the vehicle outline and the lane boundary is important to assess the vehicle motion after lane change because a large clearance allows greater lateral displacement or yaw motion.

\subsubsection{VEHICLE MOTION CRITERA AFTER LANE CHANGE}

The vehicle motion criterion after lane change is defined using the vehicle outline and lane boundaries. Any point on the vehicle outline with $x$-coordinate inside the 


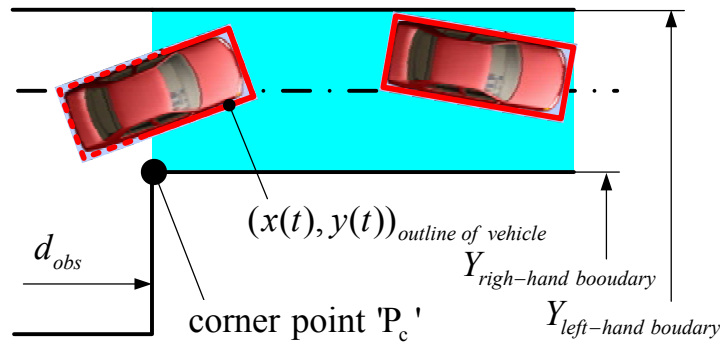

Fig. 6.4 Vehicle motion criteria after lane change

shaded area of Fig. 6.4 should stay within the lane boundaries. In other words, the vehicle motion criteria can be written

$$
\begin{aligned}
& Y_{\text {right-hand boundary }}<Y<Y_{\text {left-hand boundary }} \\
& \text { where } \\
& P_{\text {vehicle }}=\left\{(X, Y): X>d_{\text {obs }} \text { and }(X, Y) \subset(x(t), y(t))_{\text {outline of vehicle }}, t>0\right\} \\
& \text { and } 0<Y_{\text {right-hand boundary }}<Y_{\text {left-hand boundary }}
\end{aligned}
$$

Here, $P_{\text {vehicle }}$ represents a set of the vehicle outline points which $x$-coordinate is greater than the obstacle distance for all time $t$.

\subsubsection{MPC DESIGN}

Regarding actuator apportionment, the reference vehicle model for the MPC is a 3 DoF nonlinear vehicle model which consists of longitudinal, lateral and yaw motions with four wheels. The linearized first-order state-space equation at non-equilibrium points consists of the state vector $x=\left[V_{x}, V_{y}, r\right]^{T}$, the vector input of slip ratios and front steering input, $u=\left[\vec{s}_{x}, \delta_{f}\right]^{T}=\left[s_{x 1}, s_{x 2}, s_{x 3}, s_{x 4}, \delta_{f}\right]^{T}$, corresponding to the individual four wheel braking control and active front steering control, respectively. Disturbance model was not considered. The outputs corresponding to vehicle performance variables are longitudinal speed, side slip, and yaw rate, $y=\left[V_{x}, \beta, r\right]^{T}$.

For simplicity, the weighting parameters in the cost function are reduced to a series of constant diagonal matrices by applying constant values up to the prediction horizon. The following weights were chosen in this study: the weight of outputs is 
$Q_{y}=\left[Q_{V_{x}}, Q_{\beta}, Q_{r}\right]=[1,150,50]$ for longitudinal, lateral and yaw motions, the weight of control inputs is $Q_{U}=\left[Q_{s x}, Q_{\delta}\right]=[200,200]$ for braking and steering control, and the weight of the change of control input is $Q_{\Delta U}=\left[Q_{\Delta s x}, Q_{\Delta \delta}\right]=[200,200]$, respectively. Of course there is considerable freedom to choose these weights, and the values selected here were chosen based on informal tuning of responses from multiple simulations. Once determined however, they were not tuned further based on particular test conditions.

Regarding the prediction horizons $H_{P}$ and $H_{U}$, there is a trade-off between the computational load implied by a large prediction horizon, and the available improvements in control performance. Based on an earlier study (Chang and Gordon, 2007a), we chose $H_{P}=10$ and $H_{U}=1$; here $H_{U}=0$ means that the chosen control is assumed constant throughout the state prediction interval. The sample time of MPC, $T_{s}$, need not be the same as that of the measurement and can be considered as a design parameter because it is related to the choice of prediction horizon; the value $T_{s}=0.02 \mathrm{~s}$ was chosen here, again based on earlier studies. The constrained optimal solution was solved by using Matlab's optimization toolbox function, quadprog (The MathWorks, 2006a).

\subsubsection{ACTUATOR CONTROL}

The braking control constraint is intended to avoid excessive actuator demands (to protect system hardware from failure) and also to prevent wheel lock $\left(s_{x}=-1\right)$; this was translated into the form $-0.2 \leq s_{x}+\Delta s_{x} \leq 0$ in order to prevent the large slip ratio from causing a severe loss of steering controllability. Moreover, the steering control has a wheel steer angle limit, 30 degrees, and a wheel steering angle rate limit, $1.636 \mathrm{rad} / \mathrm{s}(=1500 \mathrm{deg} / \mathrm{s}$ measured at the steering wheel $)$.

For braking control, the control brake torque by the SMC is treated as an external input variable and acts on the CarSim vehicle model through a first order filter, with time 
constant $0.05 \mathrm{~s}$. Brake actuation is limited to a maximum torque of $2000 \mathrm{Nm}$ at each wheel. Similar to braking control, the steering control provided by the MPC is imported into the CarSim vehicle model through a first order transfer function with time constant $0.05 \mathrm{~s}$ and steering ratio $1 / 16$.

\subsection{SIMULATION RESULTS}

For the emergency lane change maneuver, five different control situations were simulated for comparison with the (Case 5) FICC controller:

Case 1 (No steer + ABS full braking) represents the straight maneuver with full braking by the ABS control to stop the vehicle before hitting an obstacle

Case 2 (Driver model (DM) + no braking) is to show the pure evasive maneuverability by an ideal driver without braking

Case 3 (DM + ABS full braking) and Case 4 (DM + VSC full braking) are for the evasive maneuver by an idealized driver with full braking by the ABS control and the VSC control, respectively. The VSC uses the same control logic and design parameters as that of the proposed FICC for CAS except tracking driver's intention in the upper layer and only using braking control

Case 5 (FICC including full braking), the proposed controller is implemented to perform autonomous collision avoidance maneuver.

With respect to five control situations, the simulations are performed for an obstacle distances $d_{o b s}=30 \mathrm{~m}$, and terminated when the vehicle speed is less than $1 \mathrm{~km} / \mathrm{h}$ except the case 2 due to no braking.

\subsubsection{CONTROL PERFORMANCE BY AN IDEALIZED DRIVER}

As the initial speed is increased while friction and vehicle limits are fixed, eventually the vehicle must collide with one of the boundaries. As show in Fig. 6.5-a, the 
(a) DM+ABS full braking
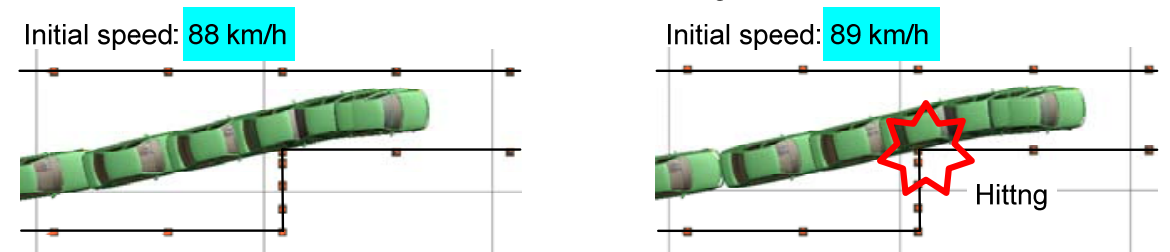

(b) DM+VSC full braking
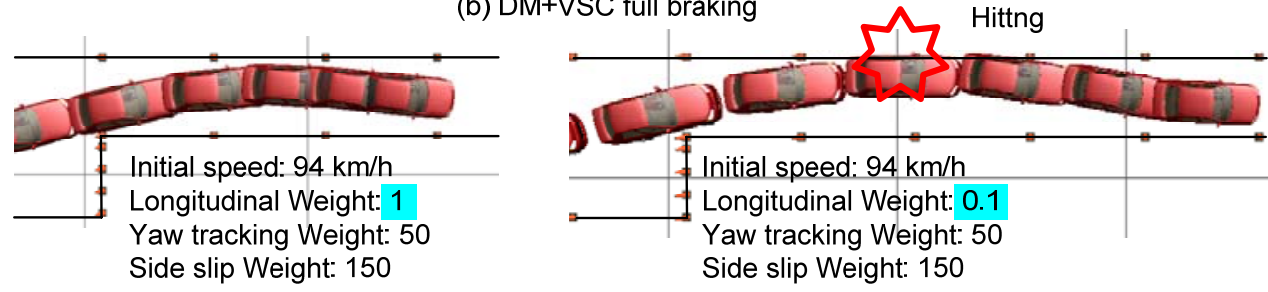

Fig. 6.5 Animation frames during emergency lane change - animation time $(\sim 2.9 \mathrm{~s})$ and time step $(0.3 \mathrm{~s})$

Case 3 control, evading an obstacle by an idealized driver model supported by only ABS control, shows that the vehicle tends towards hitting the corner point of the lane boundary as the initial vehicle speed increases. This phenomenon seems to occur because the longitudinal motion control by the ABS full braking is compromised by the lateral motion control - there is a tendency towards a straight path. On the other hand, Case 4 using VSC in Fig. 6.5-b shows the lateral motion is emphasized, giving a kind of "path over-steer" and the left lateral boundary is hit. While the details are affected by the VSC tuning parameters, the difference is qualitatively clear.

\subsubsection{CONTROLLER EVALUATION FOR COLLISION AVOIDANCE}

Fig. 6.6 shows the overall performance comparisons. The performance was summarized in terms of the maximum initial speed $U_{0}$ for which all constraints are met, as well as the sum of the individual RMS brake torques at each wheel, which can be interpreted loosely as the control effort expended in the vehicle motion. Fig. 6.7 then shows the vehicle trajectory and moving distance $d$ at the simulation terminal time $t_{s}$ during emergency lane change maneuver. 


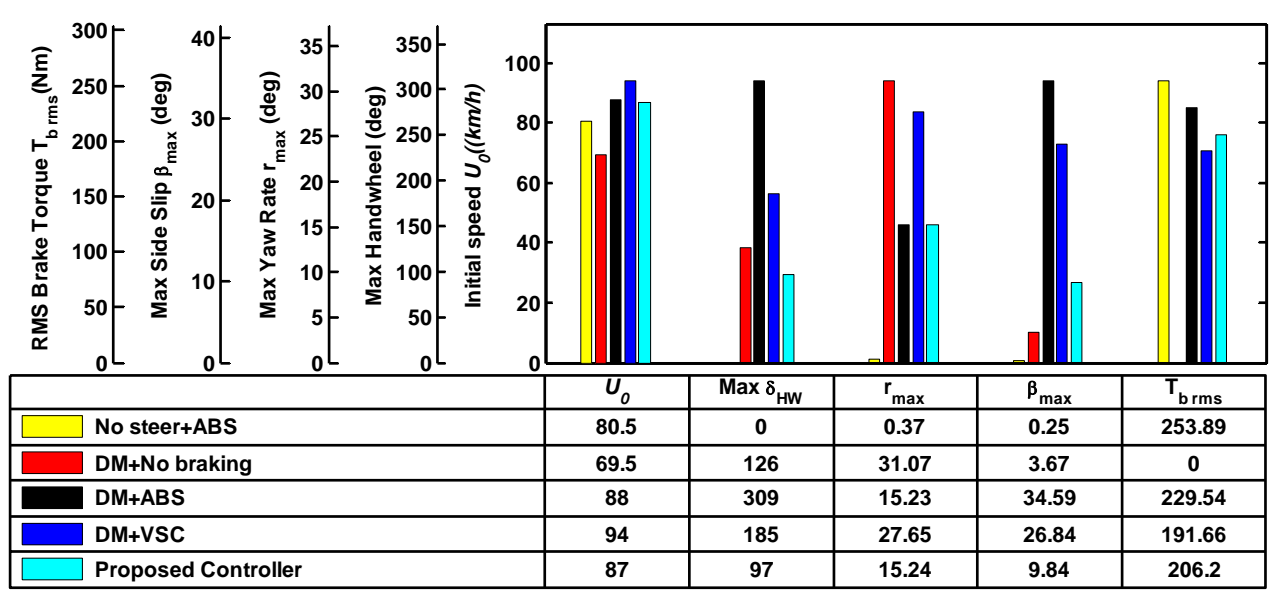

Fig. 6.6 Performance comparison for emergency lane change $\left(d_{o b s}=30 \mathrm{~m}\right)$

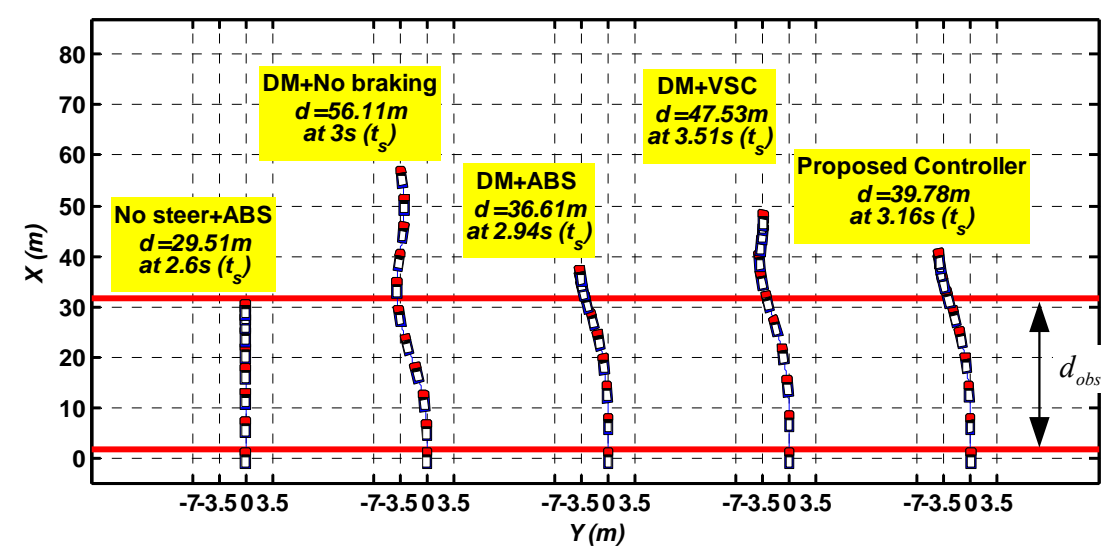

Fig. 6.7 Vehicle trajectories and moving distances $(d)$ for emergency lane change $\left(d_{o b s}=30 \mathrm{~m}\right)$

Compared to the maximum initial speed of the Case 2 (pure evading by an ideal driver without braking), the other control cases by braking show a higher initial speed. In other words, reducing the vehicle speed by braking is better stratagem to avoid collision than the pure evasive maneuver without braking. Moreover, the VSC control with an ideal driver provides better maneuverability $\left(U_{0}=94 \mathrm{~km} / \mathrm{h}\right)$ for collision avoidance than the ABS control with an ideal driver $\left(U_{0}=88 \mathrm{~km} / \mathrm{h}\right)$ by sacrificing increased moving distance $(d=47.53 \mathrm{~m})$ compared to that of the ABS control $(d=36.61 \mathrm{~m})$ as a result of the balance between the steerability and the longitudinal motion control. The FICC control gives performance that is quite similar to Cases 3 and 4 , even though it represents the 


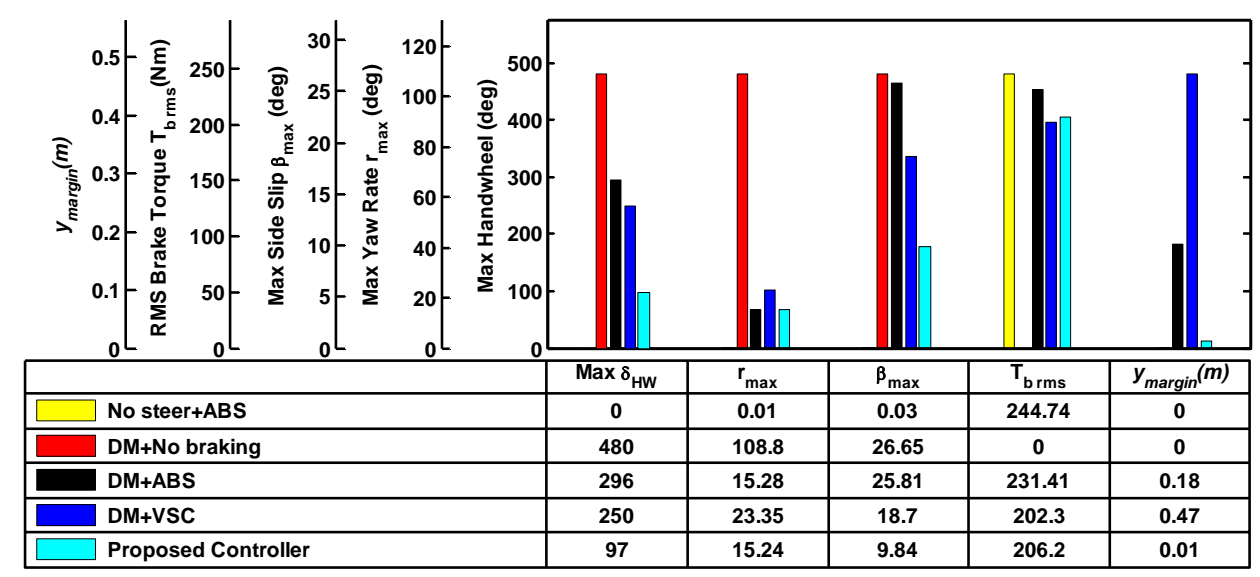

Fig. 6.8 Performance comparison for emergency lane change $\left(d_{o b s}=30 \mathrm{~m}\right)$ with a fixed initial speed $U_{0}=87 \mathrm{~km} / \mathrm{h}$

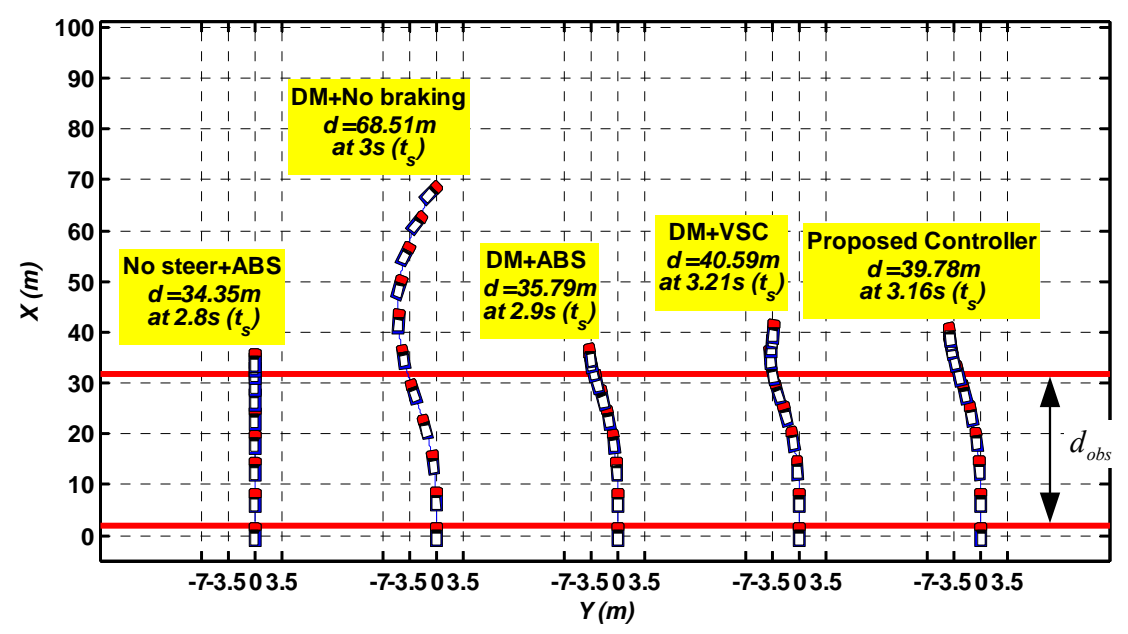

Fig. 6.9 Vehicle trajectories and moving distances $(d)$ for emergency lane change $\left(d_{o b s}=30 \mathrm{~m}\right.$, Initial vehicle speed $\left.=87 \mathrm{~km} / \mathrm{h}\right)$

automated response of the vehicle, rather than an optimized steering control by an alert and planning driver, supported by ABS or VSC. To make comparisons more meaningful however, a uniform initial speed is now chosen.

Fig. 6.8, Fig. 6.9 and Fig. 6.10 show the performance comparison, trajectory response and vehicle responses for the emergency lane change against the obstacle distance $30 \mathrm{~m}$ with the same initial speed $87 \mathrm{~km} / \mathrm{h}$, respectively. Case 1 (No steer + ABS) hits the obstacle and Case 2 (DM + no braking) becomes unstable due to spinning-out. 

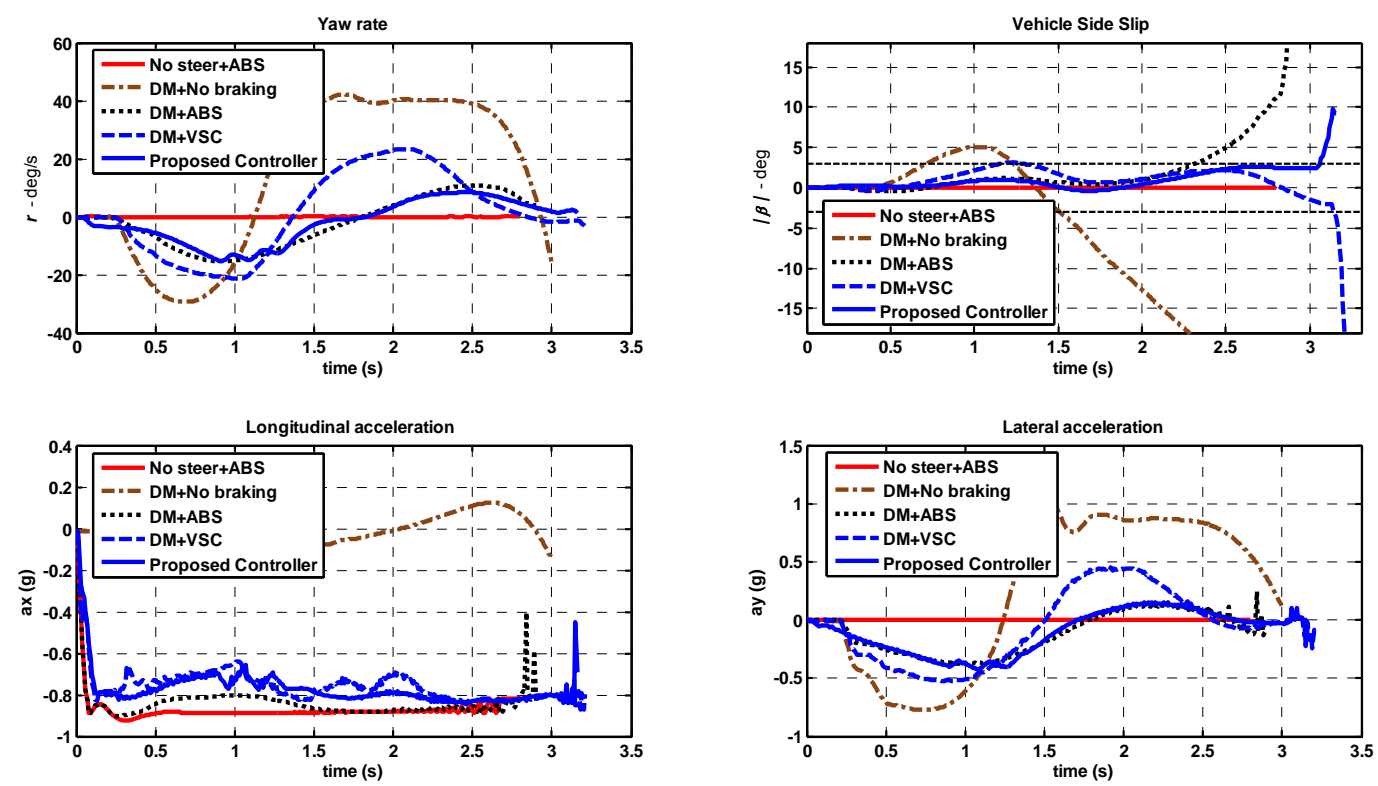

Fig. 6.10 Vehicle responses comparison for emergency lane change $\left(\mathrm{d}_{\mathrm{obs}}=30 \mathrm{~m}\right.$, Initial vehicle speed $=87 \mathrm{~km} / \mathrm{h}$ )

On the other hand, other case 3, 4 and 5 by braking control show stable motions and no collision with obstacles, so comparisons may be made. Between Cases 3 and 4 (idealized driver) the maximum handwheel steer input can be interpreted as driver's effort to perform the lane change, and the VSC control shows smaller maximum handwheel steer and larger $Y_{\text {margin }}$ (the clearance between the vehicle outline and the lane boundary) than the ABS control. This shows that the VSC control performs better steerability by sacrificing its deceleration ability compared to the ABS control. The proposed FICC still performs successfully in the emergency lane change, uses less steering authority than the driver model cases, and its vehicle dynamic responses are very similar to those of the ABS control as shown in Fig. 6.10.

\subsection{SUMMARY}

As an extension of the vehicle stability control, a hierarchical control scheme for integrated chassis control has been applied to the problem of collision avoidance system (CAS) design. This includes both lateral and longitudinal control, using Active Front 
Steer (AFS) in addition to brake actuators. The extension is realized by extending the functionality of an upper layer motion controller, and the reference model of the intermediate MPC, which is a 3 DoF nonlinear vehicle model. The vehicle motions, in the form of reference yaw rate and two-dimension mass center accelerations, are determined using a kinematic policy for collision avoidance previously proposed in the context of a driver model. The kinematic policy uses simple information about range and azimuth angles for multiple points that bound the available vehicle trajectory, and prioritizes yaw motion response based on the worst case collision threat. This makes the CAS implementation both simple and computationally inexpensive, certainly compared to a controller that must repeatedly make path planning and optimization decisions. The fact that collision avoidance is nearly as effective as the combination of an optimizing driver supported by the MPC-based stability controller suggests that this approach could be used in future high performance collision avoidance systems. 


\section{CHAPTER 7}

\section{CONCLUSIONS AND FUTURE RESEARCH SUGGESTIONS}

\subsection{CONCLUSIONS}

Integrated vehicle control for active safety systems is currently given a great deal of attention by the automotive industry in terms of performance improvement and cost reduction. Performance is dependent on control objectives such as safety, comfort, flexibility, modularity and reliability. These multiple objectives can be effectively achieved by avoiding functional conflicts between each chassis control system. Cost reduction can be simply obtained by integrating or sharing hardware such as ECUs or sensors. Costs can also be lowered by saving development time and resources. This can be achieved through a flexible control structure that covers various customer demands, subsystems suppliers, and driving conditions.

The proposed controller offers a total vehicle motion control solution in an active safety system. This control is achieved by addressing several issues of the integrated vehicle control systems design in terms of modularity, flexibility, and robustness. In this thesis, modularity is realized by the model-based hierarchical control structure that consists of three layers: an upper layer for reference vehicle motions, an intermediate layer for actuator apportionment, and a lower layer for stand-alone actuator control. Reference vehicle motions can be determined by means of any type of reference model such as steady-state bicycle model for vehicle stability control or Kinematic policy for collision avoidance control. In the intermediate layer, the actuator apportionment uses MPC to provide the inherent flexibility of the controller. Flexibility is achieved by 
simultaneously balancing tire forces to track target reference vehicle motions and considering constraint conditions such as actuator limits.

The proposed MPC design, LTV-MPC, avoids the complexity of full nonlinear MPC for real-time implementation, and addresses the vehicle nonlinearity by using timevarying linearization at arbitrary (non-equilibrium) operating points for predictive vehicle model accuracy. For the practical implementation of the MPC into vehicle control systems, several strategies for MPC predictive model were presented according to the vehicle model freedom of degree and various chassis actuators.

The proposed controller was evaluated through intensive simulation studies. The studies examined the performance of the vehicle stability control to track the reference vehicle motions while keeping vehicle stability, controller robustness against model-plant mismatch, actuator time constant, vehicle state and road surface friction estimation, and the flexibility of the controller to mitigate actuator failure. Moreover, the effects of the MPC design parameter tuning and actuator conditions were discussed, and the feasibility study of the real-time implementation was demonstrated through a pc-based real-time simulation using Matlab toolbox Real Time Simulation Target.

The evaluation of the proposed controller also explored possible trade-offs between several factors so that multiple control objectives under certain vehicle and road surface friction limits can be achieved:

- Tuning the weights of the cost function to balance multiple objectives

- Minimizing model-plant mismatch to reduce the error of vehicle motion prediction

- Adapting feasible reference vehicle motions to prevent excessive or too weak control

- Increasing the accuracy of measured signals or estimated states

- Considering actuator specification in the stand-alone lower level control in terms of actuator frequency bandwidth and maximum actuator power capacity 
Among the above control design factors, the dominant factors were the choice of the weights of the cost function and the reference vehicle motions, which are saturated by road surface friction. Thus, these two factors should be considered first in efforts to achieve more robust control.

As an extension of the vehicle stability control, the proposed controller was applied to collision avoidance systems (CAS) by adapting the reference vehicle motions at the upper level of the controller. The desired vehicle motions in the upper layer, in the form of reference yaw rate and two-dimensional mass center accelerations, are determined using a kinematic policy for collision avoidance. The performance of the controller was evaluated under emergency lane change with full braking compared to the idealized CAS driven by an optimal-based driver model. The results of this analysis show that the proposed CAS controller is nearly as effective as the combination of an optimizing driver supported by the MPC-based stability controller. This outcome suggests that this approach could be used in future high performance collision avoidance systems.

In summary, the specific major contributions of this thesis are as follows;

- An off-line advanced MPC based control system that deals with vehicle and tire nonlinearity has been implemented in software.

- Feasibility of the real-time version of the proposed controller was demonstrated.

- Flexible actuator apportionment was established to improve the efficiency of friction utilization under limited vehicle and tire conditions.

- The proposed control structure was shown to be capable of providing the required functionality for collision avoidance systems. 


\subsection{FUTURE RESEARCH SUGGESTIONS}

The framework presented in this thesis offers a framework for systematic development to address the following issues in future.

- Experimental real-time implementation tests: From the practical implementation viewpoint, the experimental tests will likely confirm the effectiveness of the proposed controller in terms of the flexible modular design as an active safety system. The appropriate hardware design should be introduced, particularly the interface between the electronic controller and vehicle mechanical systems.

- Improvement in the MPC stability on vehicle control systems: Even though vehicle control tracks time-varying set-points during severe transient maneuvers, the existing theory can be applied to the saturated region due to vehicle and road limits where the change of the time-varying set-point is not considerable. In this saturated region, stability theory can improve vehicle response from the practical viewpoint by overriding pre-defined weights of cost function. However, several challenges remain to be addressed. Specifically, more work should be done on the saturated region identification and appropriate controller tuning to avoid too aggressive control due to the direct implementation of control inputs that respect theoretical stability constraints.

- Integration of collision avoidance control with environment information devices such as radar and vision systems: More challenges can be expected in terms of robustness of realistic environment information and control design parameter tuning when specific environment information techniques are integrated to the proposed collision avoidance control.

- Decision logic of the controller mode switching as a global active vehicle safety controller: The decision for overriding driver input is still a hot issue in the 
automotive industry because a vehicle is a safe-critical system. Thus, the controller should be designed to combine multiple pre-warning steps and minimize the effects of any possible faulty operation

- Extension of target vehicle motions to develop a fully integrated chassis controller: In this thesis, integrated chassis control is limited to application in four wheel individual braking control and active front control for longitudinal, lateral and yaw motion control. The extension of chassis actuator systems and controller functionality can be achieved by modifying the predictive vehicle model of the MPC. For example, a predictive vehicle model could be constructed by adding vertical and/or roll dynamics in addition to the longitudinal, lateral and yaw motions and more additional actuators such as rear-steer, drive torque vectoring (via controllable differentials) and active or semi-active suspension. With these additions, the degrees of freedom increase for the predictive vehicle model. While this will tend to increase the computational burden, it also offers the greatest possible benefit for integrated chassis control in the future. 
APPENDICES 


\section{APPENDIX A}

\section{DOF NONLINEAR VEHICLE MODEL}

The 8 DoF nonlinear vehicle model comprises 4 DoF for rigid body dynamics and 4 DoF for wheel rotation. The vehicle body has longitudinal, lateral, yaw and roll degrees freedom-Fig. A.1. Vertical and pitch degrees of freedom are not considered and the road surface is considered flat and horizontal. The wheel dynamics include brake torque and longitudinal tire force at the contact point on the ground -Fig. A.2.

Tire rolling resistance and driveline friction are not considered in the model. However, longitudinal and lateral force equations do include aerodynamic drag forces, which are proportional to the square of the speed, in a direction that opposes the mass center velocity vector. The vehicle dynamics equations are formulated as follows.

Longitudinal:

$$
m\left(\dot{V}_{x}-r V_{y}-r p h_{1} \cos \varepsilon\right)=\sum_{i=1}^{4} F_{x i}-F_{\text {aero }} \cos \beta
$$

Lateral:

$$
m\left(\dot{V}_{y}+\dot{p} h_{1} \cos \varepsilon+r V_{x}\right)=\sum_{i=1}^{4} F_{y i}-F_{\text {aero }} \sin \beta
$$

Roll:

$$
\begin{aligned}
\sum M_{x} & =\left[\left(I_{x x}+m h_{1}^{2}\right) \cos \varepsilon-I_{x z} \sin \varepsilon\right] \dot{p}-I_{x z} \dot{r}+m\left(\dot{V}_{y}+r V_{x}\right) h_{1} \\
& =\left(m g h_{1}-K_{\varphi f}-K_{\varphi r}\right) \varphi-\left(K_{p f}+K_{p r}\right) p-\left(h_{0}-h_{r f}\right) \sum_{i=1}^{2} F_{y i}-\left(h_{0}-h_{r r}\right) \sum_{i=3}^{4} F_{y i}
\end{aligned}
$$

Yaw:

$$
\begin{aligned}
\sum M_{z} & =\left(-I_{x z} \cos \varepsilon+I_{z z} \sin \varepsilon\right) \dot{p}+I_{z z} \dot{r} \\
& =c_{f}\left(F_{x 1}-F_{x 2}\right)+c_{r}\left(F_{x 3}-F_{x 4}\right)+a\left(F_{y 1}+F_{y 2}\right)-b\left(F_{y 3}+F_{y 4}\right)
\end{aligned}
$$

Wheel rotation:

$$
I_{w i} \dot{\omega}_{i}=T_{b i}-F_{x w i} \cdot R_{w} \quad\left(i=1 \sim 4, T_{b i} \leq 0\right)
$$




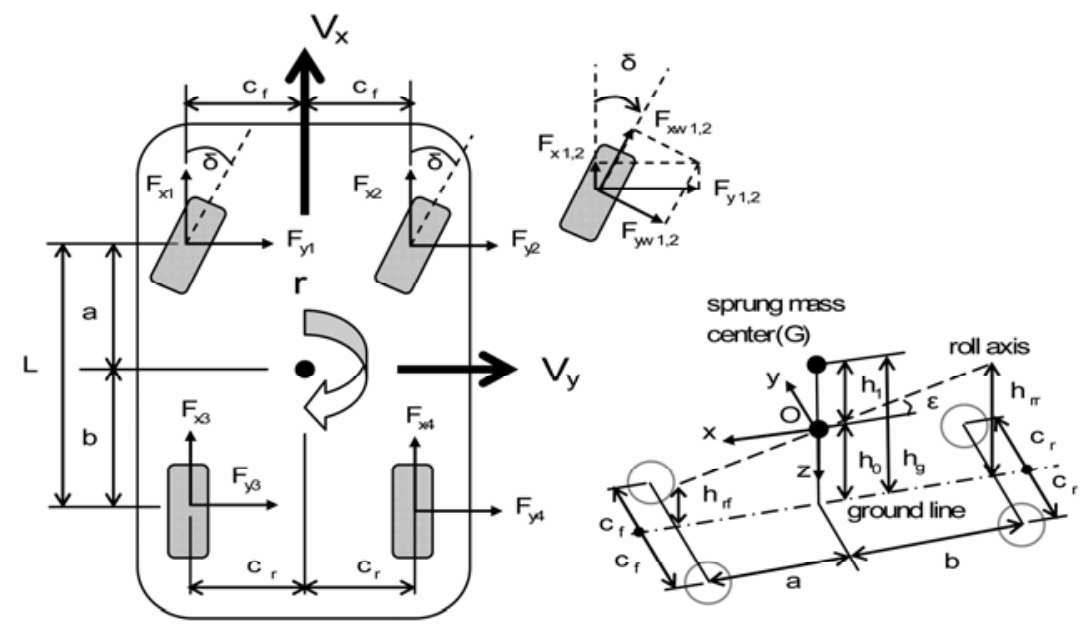

Fig. A.1 Geometric definition of the 8 DoF vehicle model in the SAE coordinate system

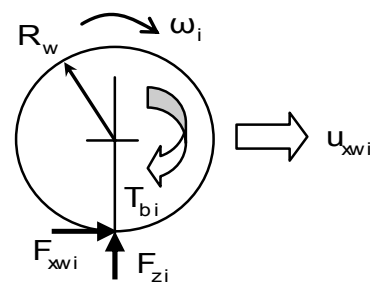

Fig. A.2 Wheel rotational dynamics

Here $\beta=\arctan \left(V_{y} / V_{x}\right)$ is vehicle sideslip angle (rad) and $\delta$ is steering angle at the front wheel (rad). $\omega_{i}$ and $T_{b i}$ are respectively angular velocity $(\mathrm{rad} / \mathrm{s})$ and brake torque $(\mathrm{Nm})$ at the $i$-th wheel. $F_{x i}, F_{y i}$ and $F_{z i}$ are tire forces in vehicle $x, y$ and $\mathrm{z}$ directions at $i$-th tire contact patch, and $F_{x w i}$ and $F_{y w i}$ are the longitudinal and lateral tire forces at $i$-th tire contact patch, with directions defined relative to the wheel plane intersection with the ground plane. $F_{\text {aero }}$ is the aerodynamic drag force on the vehicle. Further vehicle parameters are given in Appendix E.

The vertical loads are calculated by considering the static equilibrium of a vehicle mass and dynamic load transfers of the sprung mass as follows.

$$
F z_{1,2}=\frac{m g b}{2(a+b)}-\frac{h_{c g}}{2(a+b)} \sum_{i=1}^{4} F_{x i} \mp \frac{K_{\varphi f}}{2 c_{f}} \varphi \mp \frac{K_{p f}}{2 c_{f}} p \pm \frac{h_{r f}}{2 c_{f}}\left(F_{y 1}+F_{y 2}\right)
$$




$$
F z_{3,4}=\frac{m g a}{2(a+b)}+\frac{h_{c g}}{2(a+b)} \sum_{i=1}^{4} F_{x i} \mp \frac{K_{\varphi r}}{2 c_{r}} \varphi \mp \frac{K_{p r}}{2 c_{r}} p \pm \frac{h_{r r}}{2 c_{r}}\left(F_{y 3}+F_{y 4}\right)
$$

The nonlinear tire force generation is represented by the combined slip tire model based on Pacejka's Magic formula; a similarity model is used (Pacejka, 2002; Milliken and Milliken, 2003; Gordon and Best, 2006) with tire forces given as functions of tire longitudinal slip ratio $s_{x i}$, tire lateral slip ratio $s_{y i}$, vertical load $F_{z i}$ and surface friction coefficient $\mu_{s}$. The longitudinal and lateral slip ratios, $s_{x i}$ and $s_{y i}$, are defined as

$$
\begin{gathered}
s_{x i}=\left(R_{w} \omega_{i}-u_{x w i}\right) / u_{x w i} \\
s_{y i}=\tan \left(-u_{y w i} / u_{x w i}\right)
\end{gathered}
$$

where $u_{x w i}$ and $u_{y w i}$ represent the longitudinal and lateral velocity of the $i$-th wheel center. Eq. (A.9) shows the longitudinal $F_{x}$ and lateral $F_{y}$ tire forces,

$$
\begin{gathered}
F_{x}=\frac{\mu_{x} \cdot F_{z p} \cdot R\left(s_{R}\right) \cdot s_{x}}{\sqrt{s_{x}^{2}+\eta\left(s_{R}\right)^{2} \tan ^{2} \alpha}}, \\
F_{y}=\frac{\mu_{y} \cdot F_{z p} \cdot R\left(s_{R}\right) \cdot \eta \tan \alpha}{\sqrt{s_{x}^{2}+\eta\left(s_{R}\right)^{2} \tan ^{2} \alpha}}
\end{gathered}
$$

where

$$
\begin{gathered}
R\left(s_{R}\right)=D \sin \left(C \arctan \left(B(1-E) s_{R}+E \arctan \left(B s_{R}\right)\right)\right) \\
s_{R}=\sqrt{\left(\frac{C_{x} s_{x}}{\mu_{x} F_{z p}}\right)^{2}+\left(\frac{C_{y} \tan \alpha}{\mu_{y} F_{z p}}\right)^{2}} \\
\eta\left(s_{R}\right)=\left\{\begin{array}{l}
0.5\left(1+\eta_{0}\right)-0.5\left(1-\eta_{0}\right) \cos \left(0.5 s_{R}\right) \text { for } s_{R}<2 \pi \\
1 \text { for } s_{R}<2 \pi
\end{array} \text { and } \eta_{0}=\frac{C_{y} \mu_{x}}{C_{x} \mu_{y}} .\right.
\end{gathered}
$$

$\mu_{x}$ and $\mu_{y}$ are the tire longitudinal and lateral friction coefficients, and $C_{x}$ and $C_{y}$ represent the longitudinal and lateral stiffness coefficients. The peak vertical force $F_{z p}$ is approximated by the following function according to the vertical load $F_{z}$.

$$
F_{z p}\left(F_{z}\right)=\frac{F_{z}}{1+\left(1.5 F_{z} / M_{t o t} \cdot g\right)^{3}} .
$$




\section{APPENDIX B}

\section{SLIDING MODE CONTROL(SMC) FOR WHEEL SLIP CONTROL}

In SMC, a brake torque $T_{b i}$ as a control input at the $i$-th wheel is represented by an equivalent control torque $T_{b i, e q}$ and a switching control torque $T_{b i, s w}$, $T_{b i}=T_{b i, e q}+T_{b i, s w}$. The equivalent control torque can be interpreted as a control that makes the system state $\left(s_{x i}, \dot{x}_{x i}\right)$ move along the desired sliding surface in which a change of control law occurs, and is determined by the wheel dynamics Eq. (A.5) and the slip ratio Eq. (A.7). Switching control torque ensures that the trajectory of system state reaches at the desired sliding surface, and the magnitude of a switching control torque can be analytically obtained by using Lyapunov stability condition. For simplicity, the brake torque is derived by using the omission of the subscript $i$ corresponding to wheel position because the brake torque at each wheel is calculated under the same control law respectively. The more investigation of the effects of different sliding surface designs on the wheel slip controller can be found (Shim et al., 2007).

\section{B.1 EQUIVALENT CONTROL, $T_{b, e q}$}

Regarding the first conventional sliding surface design $\sigma=\tilde{x}=s_{x}-s_{x d}$, where $s_{x d}$ denotes a desired slip ratio, there is no relationship between a slip ratio and its derivative such as an exponential convergence of states on the sliding surface corresponding to the common SMC characteristic of second order systems.

For the purpose of improvement of the convergence rate, the sliding surface is designed by $\sigma=\dot{\tilde{x}}+\gamma \tilde{x}$. The equivalent torque can be calculated by the sliding condition $\sigma=0$ because the brake control input appears in this condition.

$$
\begin{aligned}
\sigma & =\dot{\tilde{x}}+\gamma \tilde{x}=\dot{s}_{x}-\dot{s}_{x d}+\gamma\left(s_{x}-s_{x d}\right) \\
& =\frac{R_{w}}{u_{x w} I_{w}}\left(T_{b}-F_{x w} R_{w}\right)-\frac{\left(1+s_{x}\right)}{u_{x w}} \dot{u}_{x w}+\gamma\left(s_{x}-s_{x d}\right)=0
\end{aligned}
$$




$$
T_{b},_{e q}=F_{x w} R_{w}+\left(1+s_{x}\right) \dot{u}_{x w} \frac{I_{w}}{R_{w}}-\gamma\left(s_{x}-s_{x d}\right) \frac{u_{x w} I_{w}}{R_{w}}
$$

In the above equation, the equivalent brake torque $T_{b, e q}$ can be replaced with an approximated equivalent control torque $\hat{T}_{b, e q}$ by considering the estimation of vehicle states and tire forces which are hard to measure directly. For the simplification of control design, tire forces and the longitudinal acceleration of each wheel center are only considered as approximated values and the uncertainty including other vehicle states and parameters such as $R_{w}, I_{w}, u_{x w}$ and $s_{x}$ is not considered.

The approximated longitudinal tire force $\hat{F}_{x w}$ can be estimated by using Kalman Filter (KF) technique or Extended Kalman Filter (EKF) technique depending on the measurement data. Four longitudinal accelerations at each wheel center $\dot{u}_{x w i}(i=1 \sim 4)$ are approximated by using the measured longitudinal/lateral acceleration and steering angle.

$$
\hat{\dot{u}}_{x w 1,2}=a_{x} \cos \delta+a_{y} \sin \delta \text { and } \hat{\dot{u}}_{x w 3,4}=a_{x}
$$

Then the approximated brake torques according to sliding surface is

$$
\hat{T}_{b}, e q=\hat{F}_{x w} R_{w}+\left(1+s_{x}\right) \hat{\dot{u}}_{x w} \frac{I_{w}}{R_{w}}-\gamma\left(s_{x}-s_{x d}\right) \frac{u_{x w} I_{w}}{R_{w}}
$$

\section{B.2 SWITCHING CONTROL, $T_{b, s w}$}

The roll of a switching control is to drive the system states to the sliding surface $(\sigma=0)$. Defining the switching control as $T_{b, s w}=-K \operatorname{sgn}(\sigma)$ where $\mathrm{K}$ is a switching control gain and $\operatorname{sgn}(\sigma)$ is a sign function, brake control torque is

$$
T_{b}=\hat{T}_{b, e q}+T_{b, s w}=\hat{T}_{b, e q}-K \operatorname{sgn}(\sigma)
$$

Considering the physical limit of the actuator, the switching gain will be chosen with satisfaction of the stability condition rather than choosing the sufficiently increasing switching gain $K$.

In order to determine the magnitude of switching control gain, Lyapunov method is applied on the slip ratio error domain using the candidate of Lyapunov function as 


$$
V=\frac{1}{2} \tilde{x}^{2}
$$

For stability, the time derivative of $V(\tilde{x})$ should be less than or equal to zero.

$$
\dot{V}=\tilde{x} \dot{\tilde{x}} \leq 0
$$

From the sliding surface equation, the above equation can be rewritten as

$$
\sigma \dot{\tilde{x}}<0 \quad \because \dot{V}=\frac{1}{\gamma}(\sigma-\dot{\tilde{x}}) \dot{\tilde{x}}=\frac{1}{\gamma} \sigma \dot{\tilde{x}}-\frac{1}{\gamma}|\dot{\tilde{x}}|^{2} \text { and }-\frac{1}{\gamma}|\dot{\tilde{x}}|^{2} \leq 0
$$

From Eqs. (B.4), (B.5) and (B.8), and letting $F=K \frac{R_{w}{ }^{2}}{u_{x w} I_{w}}$,

$$
\begin{array}{r}
\sigma \dot{\tilde{x}}=\sigma\left[\frac{R_{w}{ }^{2}}{u_{x w} I_{w}}\left(\hat{F}_{x w}-F_{x w}\right)+\frac{\left(1+s_{x}\right)}{u_{x w}}\left(\hat{\dot{u}}_{x w}-\dot{u}_{x w}\right)-\gamma\left(s_{x}-s_{x d}\right)-F \operatorname{sgn}(\sigma)\right] \leq 0 \\
\sigma\left[\frac{R_{w}{ }^{2}}{u_{x w} I_{w}}\left(\hat{F}_{x w}-F_{x w}\right)+\frac{\left(1+s_{x}\right)}{u_{x w}}\left(\hat{\dot{u}}_{x w}-\dot{u}_{x w}\right)-\gamma\left(s_{x}-s_{x d}\right)\right] \leq F|\sigma|
\end{array}
$$

Then the boundary $F$ can be obtained by applying the triangle inequality to the above equation.

$$
F \geq \frac{R_{w}^{2}}{u_{x w} I_{w}}\left|\hat{F}_{x w}-F_{x w}\right|+\frac{\left(1+s_{x}\right)}{u_{x w}}\left|\hat{\dot{u}}_{x w}-\dot{u}_{x w}\right|+\gamma\left|s_{x}-s_{x d}\right| \geq 0
$$

Then, the switching gain can be written as

$$
K \geq D_{1}+I_{w} \frac{\left(1+s_{x}\right)}{R_{w}{ }^{2}} D_{2}+\gamma \frac{u_{x w} I_{w}}{R_{w}{ }^{2}}\left|s_{x}-s_{x d}\right|
$$

where the approximation errors of $F_{x w}$ and $\dot{u}_{x w}$ are assumed that they are bounded within $D_{1}$ and $D_{2}$ as follows.

$$
\left|\hat{F}_{x w}-F_{x w}\right| \leq D_{1},\left|\hat{\dot{u}}_{x w}-\dot{u}_{x w}\right| \leq D_{2}
$$

In general, $D_{1}$ and $D_{2}$ are considered as design parameters except that $F_{x w}$, and $\dot{u}_{x w}$ are precisely known, and the smaller boundaries means more expensive estimations for the exact values. Thus, in the robust design viewpoint, we suppose that these approximation boundaries are proportional to the maximum error percentage of the estimation values as follows.

$$
D_{1}=d_{1}\left|\hat{F}_{x w}\right|, D_{2}=d_{2}\left|\hat{\dot{u}}_{x w}\right|
$$


where $d_{1}$ and $d_{2}$ represent the maximum error percentage of a longitudinal tire force and the acceleration of a wheel center, respectively.

In addition, in order to avoid the chattering problem due to the imperfect switching control under the physical limits of actuator or model uncertainty, the sign function is substituted to the following saturation function with the boundary layer thickness $\Phi$ around the sliding surface.

$$
\operatorname{sat}\left(\frac{\sigma}{\Phi}\right)= \begin{cases}\operatorname{sgn}(\sigma) & \text { if }|\sigma| \geq \Phi \\ \frac{\sigma}{\Phi} & \text { otherwise }\end{cases}
$$

The control torque input can be summarized as follows:

$$
T_{b}=\hat{F}_{x w} R_{w}+\left(1+s_{x}\right) \hat{\dot{u}}_{x w} \frac{I_{w}}{R_{w}}-\gamma\left(s_{x}-s_{x d}\right) \frac{u_{x w} I_{w}}{R_{w}}-K \operatorname{sat}\left(\frac{\sigma}{\Phi}\right)
$$

where

$$
\begin{aligned}
& K \geq D_{1}+I_{w} \frac{\left(1+s_{x}\right)}{R_{w}{ }^{2}} D_{2}+\gamma \frac{u_{x w} I_{w}}{R_{w}{ }^{2}}\left|s_{x}-s_{x d}\right| \\
& D_{1}=d_{1}\left|\hat{F}_{x w}\right| \geq\left|\hat{F}_{x w}-F_{x w}\right|, D_{2}=d_{2}\left|\hat{\dot{u}}_{x w}\right| \geq\left|\hat{\dot{u}}_{x w}-\dot{u}_{x w}\right| \\
& \gamma, \Phi, d_{1} \text { and } d_{2} \text { are design parameters. }
\end{aligned}
$$




\section{APPENDIX C \\ RULE-BASED REFENCE CONTROLLER DESIGN}

A reference rule-based VSC distribution control is designed to correct the oversteer vehicle response experienced by the uncontrolled vehicle during the sinusoidal steer maneuver. This prescribes the application of brake torque at the front outer wheel of turn, or potentially releasing brake torque on the inner wheel. The controller follows the same general hierarchical control strategy as VSC, and again consists of three levels. The lower level is completely unchanged, while the intermediate level brake distribution control determines the control action at the front wheels via a proportional control, applied according to the heuristic that the magnitude of the yaw moment at each wheel is proportional to slip ratio. The upper level is very similar to that used above, except that a desired yaw moment is the output, in place of the reference vehicle motions used previously. The control algorithms for the upper and intermediate levels are now presented:

\section{Upper Level: Vehicle motion control}

a. Calculate the corrective yaw moment for desired yaw rate tracking

$$
\begin{aligned}
& \text { If }\left|r-r_{d}\right| \geq r_{\text {threshold }} \text { and }\left|r-r_{d}\right|>\left|r_{d}\right| \cdot r_{\text {threshold_percent }} \text { then } M_{z_{-} \text {yaw }}=K_{\text {yaw }} \cdot\left(r-r_{d}\right) \\
& \text { else } M_{z_{-} \text {yaw }}=0
\end{aligned}
$$

b. Calculate the corrective yaw moment for sideslip regulation

$$
\begin{aligned}
& \text { If }|\beta| \geq \beta_{\text {threshold }} \text { and } \beta \cdot \Delta \beta>0, \quad M_{z_{-} \beta}=K_{\beta p} \cdot \beta+K_{\beta d} \cdot \Delta \beta \\
& \text { else, } M_{z_{-} \beta}=0
\end{aligned}
$$

c. Apply higher priority to the sideslip regulation

$$
\text { If } M_{z_{-} \beta} \neq 0 \text { then } M_{z_{\text {desired }}}=M_{z_{-} \beta} \text {, else } M_{z_{\text {desired }}}=M_{z_{-} \text {yaw }}
$$

Intermediate Level: Brake torque distribution control 
a. Calculate a brake release command:

$$
\text { If }\left|T_{b}\right|>0 \text { then } \Delta s_{x R}=K_{R}\left|s_{x R}\right|, \quad \text { else } \Delta s_{x R}=0 \quad\left(K_{R}=T_{s} / 0.01\right)
$$

b. Calculate a brake activation command:

$$
\begin{aligned}
& \left|\Delta s_{x A}\right|=\left|M_{z \text { desired }}\right| / K_{m z}-\left|\Delta s_{x R}\right| \quad \text { where }\left|M_{z \text { desired }}\right|=K_{m z}\left(\left|\Delta s_{x A}\right|+\left|\Delta s_{x R}\right|\right) \\
& \text { If }\left|\Delta s_{x A}\right|>0 \text { then } \Delta s_{x A}=-\left(\left|M_{z \text { desired }}\right| / K_{m z}-\left|\Delta s_{x R}\right|\right), \quad \text { Else } \Delta s_{x A}=0
\end{aligned}
$$

c. Saturate the desired slip ratio if necessary:

$$
\text { If }\left|s_{x A}\right|>\left|s_{x \text { Limit }}\right| \text { then } \Delta s_{x A}=-\left(\left|s_{x \text { Limit }}\right|-\left|s_{x}\right|\right) \quad\left(s_{x A}=s_{x}+\Delta s_{x A}\right)
$$

The following design parameters are required by the rule-based reference controller: $r_{\text {threshold_percent }}, \beta_{\text {threshold }}, s_{x \text { Limit }}, K_{\text {yaw }}, K_{\beta p}, K_{\beta d}$ and $K_{m z}$; the subscript $A$ (for Activate) represents the outer wheel of turn to activate brake torque and the subscript $R$ (for Release) describes the inner wheel of turn to release brake torque. The slip ratio limit $s_{x \text { Limit }}$ is aimed to prevent the desired slip ratio from becoming excessive. Regarding these design parameter values, the reference controller uses the same thresholds of the vehicle motion control and the same design parameters of the wheel slip control as those of the MPC controller. The detailed design parameters are given in Table E.3. 


\section{APPENDIX D}

\section{PC-BASED REAL-TIME SIMULATON}

This section presents the procedure of the PC-based real-time simulation as a feasibility study of the real-time implementation. This study is achieved under Matlab/Simulink environment using Matlab toolbox Real Time Simulation Target. (The MathWorks, 2006b)

\section{D.1 CONFIGURATION OF SIMULINK BLOCK, C S-FUNCTION}

In order to use C Mex S-function in Simulink, the controller should be first written by C-programming language and compiled successfully into DLL (dynamic link library) files. Then 'C S-function' block in Simulink should be set up like Fig. D.1 which can be chosen by clicking the right-button of the mouse on the Simulink screen.

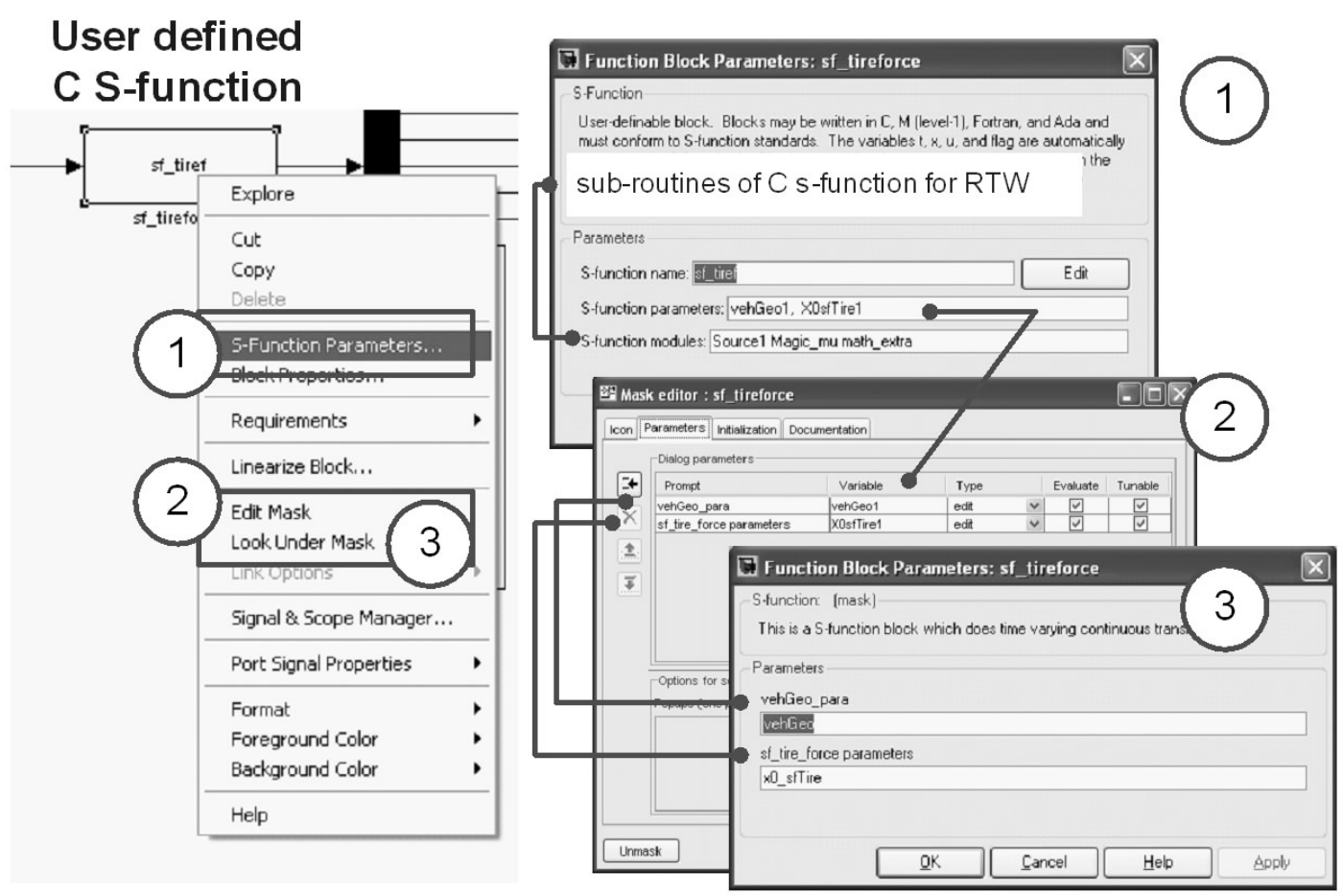

Fig. D.1 'C S-function' block set-up 
Here, the S-function name in the mark 1 corresponds to the DLL file name, and the head files of the DLL file should be defined in the S-function modules of the mark 1. The controller design parameters are defined at the S-function parameters in the mark 1, Edit Mask in the mark 2, and Look under Mask in the mark 3.

\section{D.2 PROCEDURE TO RUN REAL-TIME APPLICATION}

After the set-up of the C S-function is ready, the real-time simulation can be performed by following the procedures given in the figures from Fig. D.2 to Fig. D.5. More details can be found in (The MathWorks, 2006b).
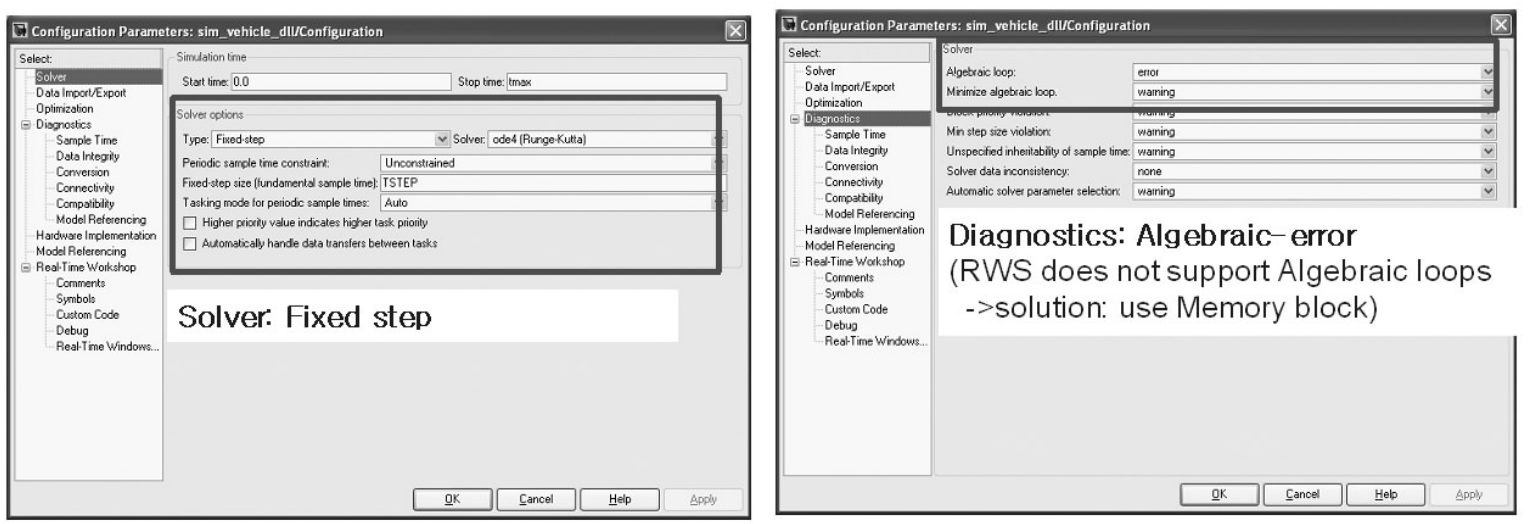

Fig. D.2 Configure the parameters of Simulink solver from the 'Simulation' popup menu in Simulink (Step 1)
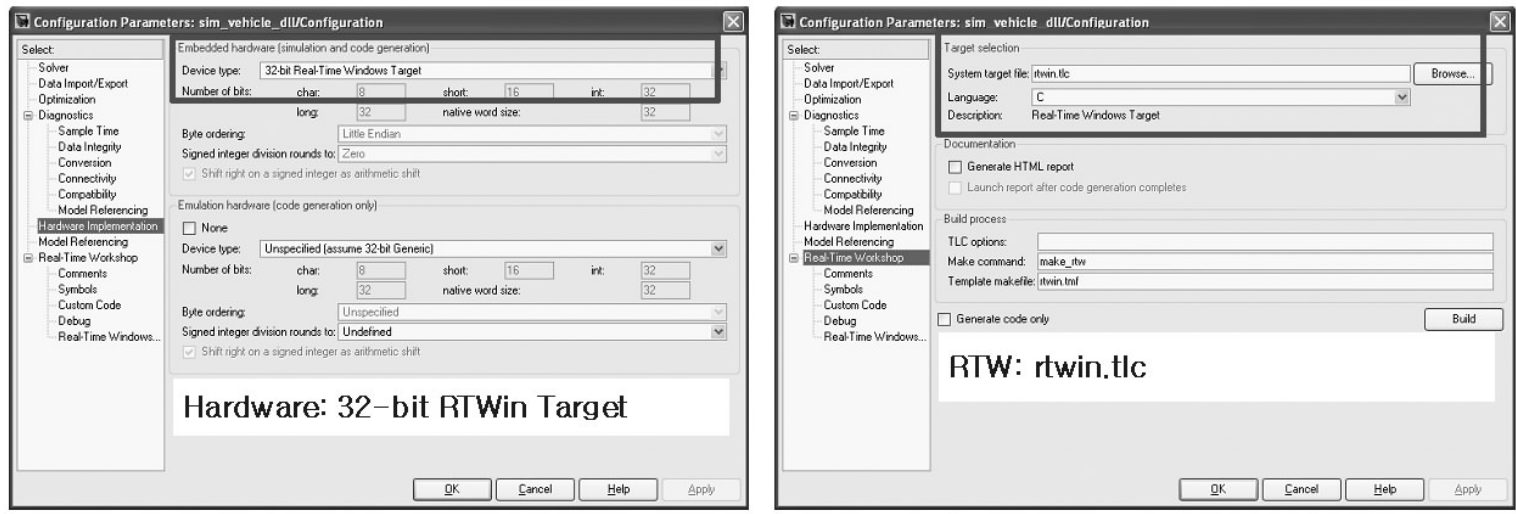

Fig. D.3 Configure the parameters of Hardware implementation and Real-time workshop from the 'Simulation' popup menu in Simulink (Step 2) 


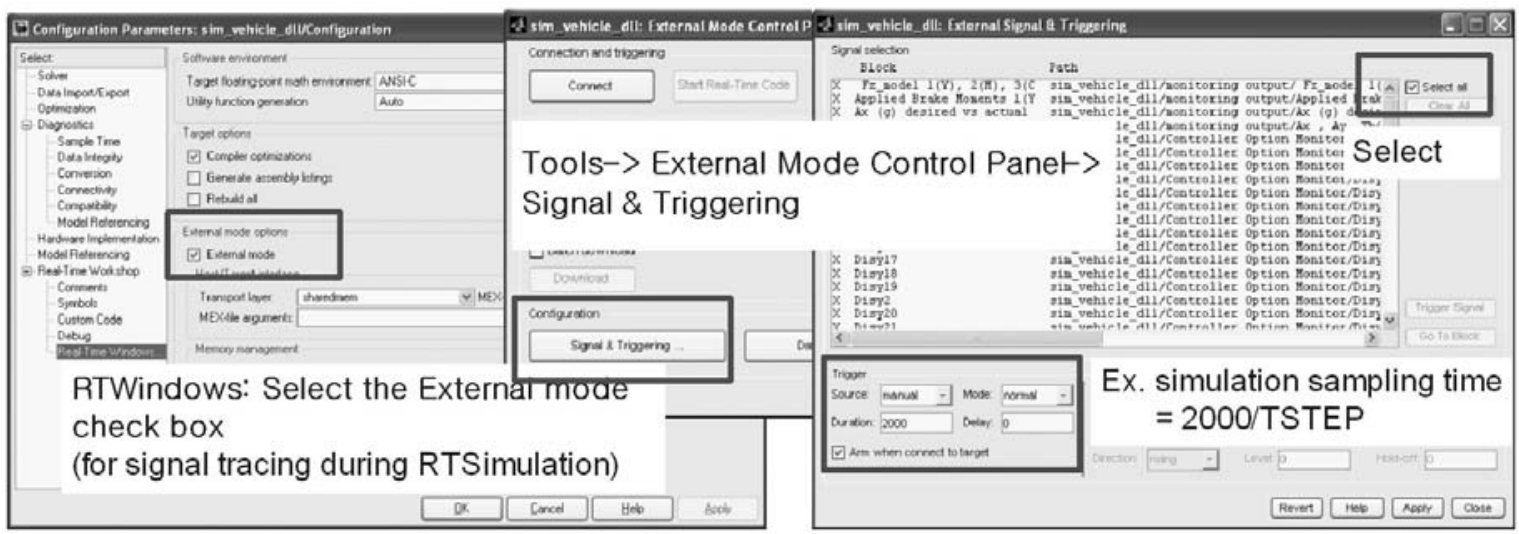

Fig. D.4 Configure the parameters of signal monitoring from 'Tools' popup menu in Simulink (Step 3)
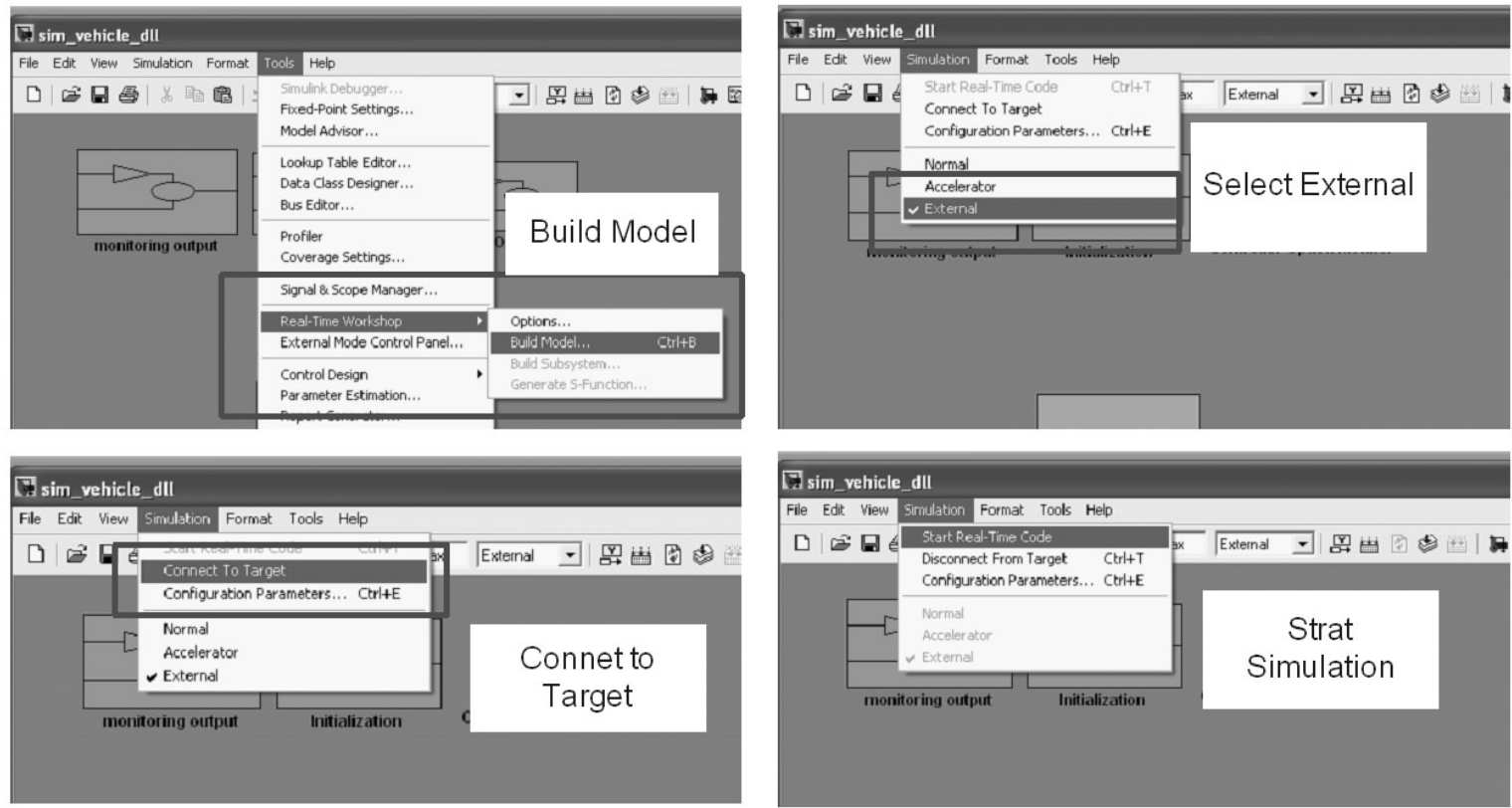

Fig. D.5 Build a real-time executable model and run simulation from 'Tools' popup menu in Simulink (Step 4) 


\section{APPENDIX E \\ LIST OF SIMULATION STUDY PARAMETERS}

\section{E.1 LIST OF VEHICLE MODEL PARAMETERS}

The reference vehicle parameters in the 8 DoF model are based on those of the CarSim "big sedan" model as follows.

Table E.1 Vehicle parameters

\begin{tabular}{ccc}
\hline$m$ & vehicle sprung mass & $1527 \mathrm{~kg}$ \\
$\mathrm{~g}$ & gravitational acceleration & $9.81 \mathrm{~m} / \mathrm{s}^{2}$ \\
$I_{x x}$ & roll moment of inertia w.r.t. x-axis & $606.1 \mathrm{~kg}-\mathrm{m}^{2}$ \\
$I_{z z}$ & yaw moment of inertia w.r.t. z-axis & $2741.9 \mathrm{~kg}-\mathrm{m}^{2}$ \\
$I_{x z}$ & product of inertia w.r.t. x and z-axis & $0 \mathrm{~kg}-\mathrm{m}^{2}$ \\
$a$ & distance of c.g to front axle & $1.014 \mathrm{~m}$ \\
$b$ & distance of c.g to rear axle & $1.676 \mathrm{~m}$ \\
$c_{f}, c_{r}$ & half track of front and rear axle & $(0.77,0.77) \mathrm{m}$ \\
$h_{c g}$ & c.g height above ground & $0.542 \mathrm{~m}$ \\
$h_{0}$ & ground to roll axis distance below c.g & $0.085 \mathrm{~m}$ \\
$h_{1}$ & distance of $h_{c g}$ to $h_{0}$ & $0.4569 \mathrm{~m}$ \\
$h_{r f}$ & height of front roll center above ground & $0.07 \mathrm{~m}$ \\
$h_{r r}$ & height of rear roll center above ground & $0.11 \mathrm{~m}$ \\
$\varepsilon$ & inclined angle between roll axis and x-axis & $0.852 \mathrm{deg}$ \\
$K_{\varphi f}, K_{\varphi r}$ & front and rear roll stiffness & $\left(5.08 \times 10^{4}, 3.83 \times 10^{4}\right) \mathrm{N}-\mathrm{m} / \mathrm{rad}$ \\
$K_{p f}, K_{p r}$ & front and rear damping rate & $\left(5.76 \times 10^{4}, 5.76 \times 10^{4}\right) \mathrm{N}-\mathrm{m}-\mathrm{s} / \mathrm{rad}$ \\
$I_{w i}$ & $i$-th wheel rotational inertia & $0.9 \mathrm{~kg}-\mathrm{m}^{2}$ \\
$R_{w}$ & effective wheel rolling radius & $0.301 \mathrm{~m}$ \\
$\mu_{s}$ & surface friction coefficient & 0.89 \\
$\tau_{t}$ & tire force build-up time constant & $0.01 \mathrm{sec}$ \\
$\tau_{b}$ & brake torque build-up time constant & $0.05 \mathrm{sec}$ \\
\hline
\end{tabular}

\section{E.2 LIST OF CONTROL DESIGN PARAMETERS}


Table E.2 Design parameters of the MPC controller

\begin{tabular}{|c|c|c|c|c|}
\hline & \multirow{2}{*}{ Design Parameter } & \multicolumn{2}{|c|}{ Controller } & \multirow{2}{*}{ Remark } \\
\hline & & LTI-MPC & LTV-MPC & \\
\hline \multirow{6}{*}{$\begin{array}{c}\text { I. Vehicle } \\
\text { motion control }\end{array}$} & $r_{\text {threshold }}$ & \multicolumn{2}{|c|}{0.5} & $\mathrm{deg} / \mathrm{sec}$ \\
\hline & $r_{\text {threshold_percent }}$ & \multicolumn{2}{|c|}{2} & $\%$ \\
\hline & $\beta_{\text {threshold }}$ & \multicolumn{2}{|c|}{3} & $\operatorname{deg}$ \\
\hline & $a_{\text {threshold }}$ & \multicolumn{2}{|c|}{0.1} & $\mathrm{~m} / \mathrm{s}^{2}$ \\
\hline & $K_{P \text { accel }}$ & \multicolumn{2}{|c|}{3} & \\
\hline & $K_{u s}$ & \multicolumn{2}{|c|}{0} & $\mathrm{rad} /(\mathrm{m} / \mathrm{s})$ \\
\hline \multirow{5}{*}{$\begin{array}{l}\text { II. Brake torque } \\
\text { distribution }\end{array}$} & $T_{s}$ & \multicolumn{2}{|c|}{0.02} & MPC sample time (s) \\
\hline & $H_{P}, H_{U}, H_{W}$ & \multicolumn{2}{|c|}{$(10 / 1 / 10)$} & Basic MPC design paramters \\
\hline & $Q_{V x}, Q_{\beta}, Q_{r}$ & $(1 / 1000 / 77.6)$ & $(1 / 300 / 3.11)$ & Output tracking error weight \\
\hline & $Q_{s x}, Q_{\Delta s x}$ & \multicolumn{2}{|c|}{$50 / 200$} & Braking control input weight \\
\hline & $Q_{\delta}, Q_{\Delta \delta}$ & \multicolumn{2}{|c|}{$50 / 200$} & AFS control input weight \\
\hline \multirow{4}{*}{$\begin{array}{l}\text { III. Wheel slip } \\
\text { control }\end{array}$} & $\gamma$ (front/rear) & \multicolumn{2}{|c|}{$103.4 / 103.1$} & \\
\hline & $\Phi$ & \multicolumn{2}{|c|}{2.585} & \\
\hline & $d_{1}$ & \multicolumn{2}{|c|}{0.5} & \\
\hline & $d_{2}$ & \multicolumn{2}{|c|}{0.5} & \\
\hline
\end{tabular}

Table E.3 Design parameters of the rule-based controller

\begin{tabular}{|c|c|c|c|}
\hline & Design Parameter & & Unit \\
\hline \hline \multirow{4}{*}{$\begin{array}{c}\text { I. Vehicle } \\
\text { motion control }\end{array}$} & $r_{\text {threshold }}$ & 0.5 & $\mathrm{deg} / \mathrm{sec}$ \\
\cline { 2 - 4 } & $r_{\text {threshold percent }}$ & 2 & $\%$ \\
\cline { 2 - 4 } & $\beta_{\text {threshold }}$ & 3 & $\mathrm{deg}$ \\
\cline { 2 - 4 } & $K_{\text {us }}$ & 0 & $\mathrm{rad} /(\mathrm{m} / \mathrm{sec})$ \\
\cline { 2 - 4 } & $K_{\text {yaw }}$ & 2.00 & $\mathrm{Nm} / \mathrm{rad} / \mathrm{sec})$ \\
\cline { 2 - 4 } & $K_{\beta p}$ & 0.77 & $\mathrm{Nm} / \mathrm{rad}$ \\
\hline \multirow{4}{*}{$\begin{array}{c}\text { II. Brake torque } \\
\text { distribution }\end{array}$} & $K_{\beta d}$ & 0 & $\mathrm{Nm} / \mathrm{rad}$ \\
\hline \multirow{4}{*}{$\begin{array}{l}\text { III. Wheel slip } \\
\text { control }\end{array}$} & $K_{\text {mz }}$ & 1 & $\mathrm{Nm} / \mathrm{slip}$ \\
\cline { 2 - 4 } & $\gamma($ front/rear $)$ & -0.2 & $\mathrm{slip}$ \\
\cline { 2 - 4 } & $\Phi$ & $103.4 / 103.1$ & \\
\cline { 2 - 4 } & $d_{1}$ & 2.585 & \\
\cline { 2 - 4 } & $d_{2}$ & 0.5 & \\
\hline
\end{tabular}




\section{E.3 LIST OF THE FICC DATA FLOW}

Fig. E.1 shows the data flow of the overall FICC control system in terms of inputs and outputs of each module. This control system covers the full functionality of the proposed control structure, including the three layers of the FICC, EKF estimation, road surface friction adaptation, kinematic policy for CAS. The details of data flow are given in Table E.4.

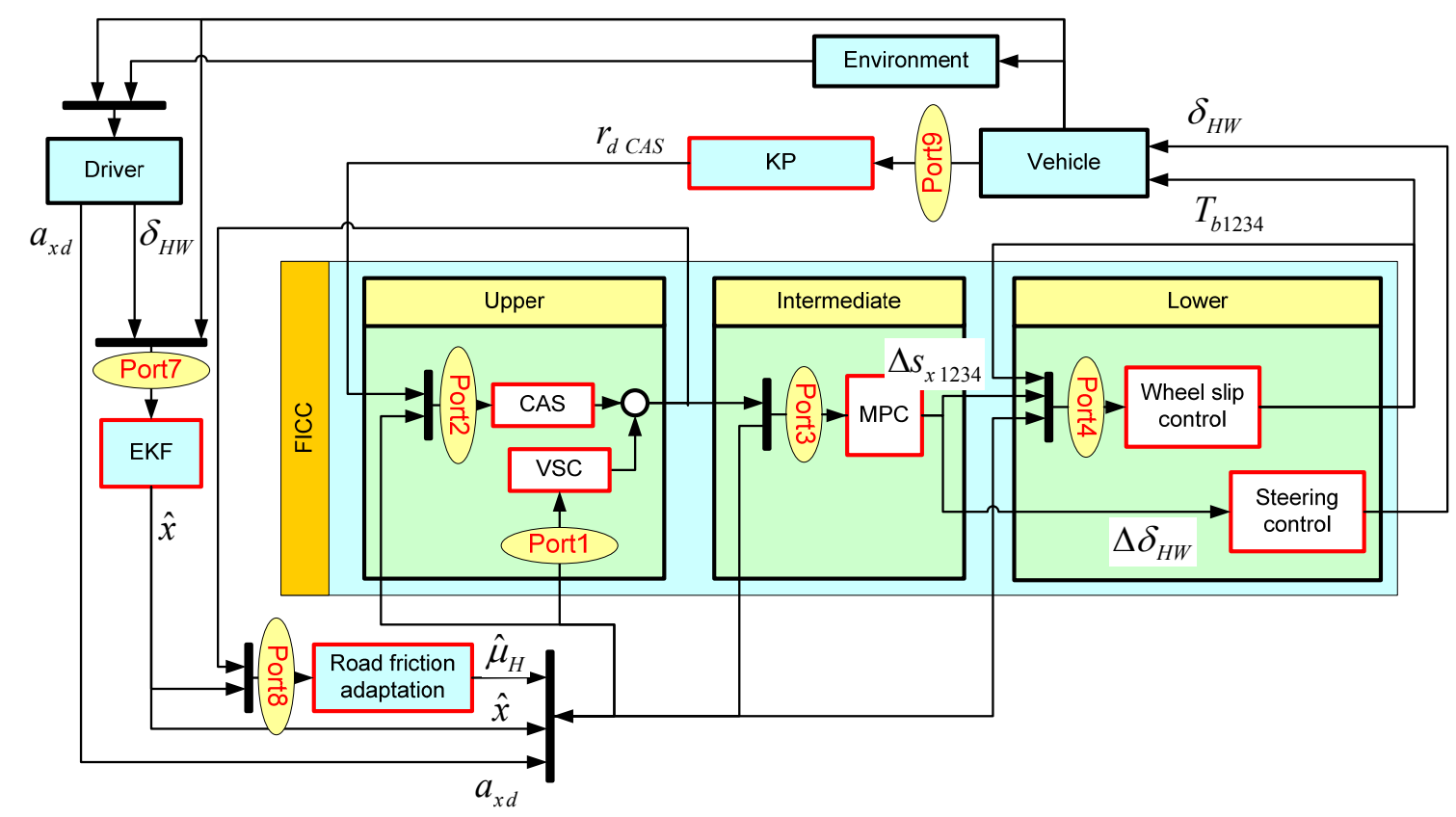

Fig. E.1 Block diagram of the FICC system 
Table E.4 List of the inputs and outputs of each module in the FICC system

\begin{tabular}{|c|c|c|}
\hline \multicolumn{2}{|c|}{ FICC module } & Remark \\
\hline \multicolumn{2}{|c|}{ Upper layer (VSC) } & \\
\hline \multirow{2}{*}{$\begin{array}{ll}\text { Input } & \text { (measurement/estimation) } \\
& \text { (control signal) } \\
\end{array}$} & $r, a_{x}, a_{y}, \delta_{H W}, V_{x}, V_{y}, \mu_{H}$ & \multirow[t]{2}{*}{ Port1 } \\
\hline & $a_{x d}$ & \\
\hline Output & $r_{d}, \beta_{d}, V_{x d}$, Controller OnOff $V_{V_{x} / \beta / r}$ flag & \\
\hline \multicolumn{2}{|c|}{ Upper layer (CAS) } & \\
\hline \multirow{2}{*}{$\begin{array}{ll}\text { Input } & \text { (measurement/estimation) } \\
& \text { (control signal) } \\
\end{array}$} & $r, a_{x}, a_{y}, V_{x}, V_{y}, \mu_{H}$ & \multirow[t]{2}{*}{ Port2 } \\
\hline & $a_{x d}, r_{d C A S}$ & \\
\hline Output & $r_{d}, \beta_{d}, V_{x d}$, Controller OnOff $V_{V_{x} / \beta / r}$ flag & \\
\hline \multicolumn{2}{|c|}{ Intermediate layer (MPC) } & \\
\hline \multirow{2}{*}{$\begin{array}{ll}\text { Input } & \text { (measurement/estimation) } \\
& \text { (control signal) }\end{array}$} & $r, V_{x}, V_{y}, \delta_{H W}, F_{z 1234}, \mu_{H}, \omega_{1234}$ & \multirow[t]{2}{*}{ Port3 } \\
\hline & $r_{d}, \beta_{d}, V_{x d}$, Controller OnOff $V_{V_{x} / \beta / r}$ flag & \\
\hline Output & $\Delta s_{x 1234}, \Delta \delta_{H W}$ & \\
\hline \multicolumn{2}{|c|}{ Lower layer (SMC) } & \\
\hline \multirow{2}{*}{$\begin{array}{ll}\text { Input } & \text { (measurement/estimation) } \\
& \text { (control signal) }\end{array}$} & $a_{x w 1234}, T_{b 1234}, F_{x w 1234}, u_{x w 1234}, s_{x 1234}$ & \multirow[t]{2}{*}{ Port4 } \\
\hline & $\Delta s_{x 1234}$ & \\
\hline Output & $T_{b 1234}$ & \\
\hline \multicolumn{2}{|c|}{ Auxiliary modules } & \\
\hline \multicolumn{2}{|c|}{ EKF module } & \\
\hline Input (measurement/estimation) & $r, p, a_{x}, a_{y}, \omega_{1234}, \delta_{H W}, T_{b 1234}$ & Port7 \\
\hline Output & $V_{x}, V_{y}, \dot{\phi}, r, \phi, \omega_{1234}, F_{x w 1234}, F_{y w \text { front }}, F_{y w \text { rear }}$ & \\
\hline \multicolumn{2}{|c|}{ Road surface friction adaptation module } & \\
\hline \multirow{2}{*}{$\begin{array}{ll}\text { Input } & \text { (measurement/estimation) } \\
& \text { (control signal) } \\
\end{array}$} & $r, a_{x}, a_{y}, \delta_{H W}$ & \multirow[t]{2}{*}{ Port8 } \\
\hline & $r_{d}$, Controller OnOff $V_{V_{x} / \beta / r}$ flag & \\
\hline Output & $\hat{\mu}_{H}$ & \\
\hline \multicolumn{2}{|c|}{ KP module } & \\
\hline Input (measurement/estimation) & $d_{i}, \phi_{i}, V_{x}, V_{y}, r$ & Port9 \\
\hline Output & $r_{d C A S}$ & \\
\hline
\end{tabular}


BIBLIOGRAPHY 


\section{BIBLIOGRAPHY}

[1] Allgower, F., Findeisen, R., and Nagy, Z. K. (2004). "Nonlinear model predictive control: From theory to application." Journal of Chinese Institute of Chemical Engineers, 35 (3), 299-315.

[2] Amberkar, S., Bolourchi, F., Demerly, J., and Millsap, S. "A Control System Methodology for Steer by Wire Systems." SAE Technical Paper No 2004-011106.

[3] Beutnagel-Buchner, U., Knoll, P. M., Schaefer, B. J., Guettler, H., Bunse, M., and Kallenbach, R. "Predictive Safety Systems-Steps Towards Collision Mitigation." SAE Technical Paper No 2004-01-1111.

[4] Bevly, D. M. (2004). "Global Positioning System (GPS): a low-cost velocity sensor for correcting inertial sensor errors on ground vehicles." ASME Journal of Dynamic Systems, Measurement, and Control, 126(2), 255-64.

[5] Borrelli, F., Falcone, P., Keviczky, T., Asgari, J., and Hrovat, D. (2005). "MPCbased approach to active steering for autonomous vehicle systems." Int. J. of Vehicle Autonomous Systems, 3(2-4), 265-291.

[6] Bosch, R. (2004). Automotive handbook, Robert Bosch GmbH

[7] Canudas-de-Wit, C., and Horowitz, R. "Observers for tire/road contact friction using only wheel angular velocity information." Phoenix, AZ, USA, 3932-7.

[8] Carlson, C. R., and Gerdes, J. C. "Optimal rollover prevention with steer by wire and differential braking." Washington, DC., United States, 345-354.

[9] Chang, S., and Gordon, T. J. "A Predictive Control For Optimal Brake Torque Distribution In Vehicle Stability Control." Proceedings of AVEC 2006.

[10] Chang, S., and Gordon, T. J. (2007a). "Model-based predictive control of vehicle dynamics." Int. J. of Vehicle Autonomous Systems, 5(1/2), 3-27.

[11] Chang, S., and Gordon, T. J. (2007b). "On the Performance Benefits of Vehicle Stability Control Using Optimal Braking Torque Distribution " JSAE Review, submitted June 2007.

[12] Chang, S., and Gordon, T. J. "A Flexible Hierarchical Model-Based Control Methodology for Vehicle Active Safety Systems." IAVSD 20th Symposium: Dynamics of Vehicles on Roads and Tracks, Berkeley, CA, August 13-17, 2007.

[13] Chen, H., and Allgower, F. (1998). "Quasi-infinite horizon nonlinear model predictive control scheme with guaranteed stability." Automatica, 34(10), 12051217.

[14] Chisci, L., Falugi, P., and Zappa, G. (2003). "Gain-scheduling MPC of nonlinear systems." International Journal of Robust and Nonlinear Control, 13(3-4), 295308. 
[15] de Oliveira Kothare, S. L., and Morari, M. (2000). "Contractive model predictive control for constrained nonlinear systems." IEEE Transactions on Automatic Control, 45(6), 1053-1071.

[16] Eckert, A. (1998). "Process for controlling driving stability with a yaw rate sensor equipped with two lateral acceleration meters." U.S. Patent 5732377.

[17] Economou, J. T., and Colyer, R. E. "Modelling of skid steering and fuzzy logic vehicle ground interaction." Chicago, IL, USA, 100-104.

[18] Falcone, P., Borrelli, F., Asgari, J., Tseng, H. E., and Hrovat, D. (2007a). "Predictive active steering control for autonomous vehicle systems." IEEE Transactions on Control Systems Technology, 15(3), 566-580.

[19] Falcone, P., Borrelli, F., Asgari, J., Tseng, H. E., and Hrovat, D. "MPC-based yaw stabilization via active front steering and braking." IAVSD 20th Symposium: Dynamics of Vehicles on Roads and Tracks, Berkeley, CA, August 13-17, 2007.

[20] Findeisen, R. (2005). "Nonlinear Model Predictive Control: A Sampled-Data Feedback Perspective," Ph.D. thesis, VDI Verlag, Dusseldorf.

[21] Fruechte, R. D., Karmel, A. M., Rillings, J. H., Schilke, N. A., Boustany, N. M., and Repa, B. S. "Integrated vehicle control." Vehicular Technology Conference, 1989 IEEE 39th, 868-877 vol.2.

[22] Ghoneim, Y. A., Lin, W. C., Sidlosky, D. M., Chen, H. H., Chin, Y.-K., and Tedrake, M. J. (2000). "Integrated chassis control system to enhance vehicle stability." International Journal of Vehicle Design, 23(1-2), 124-144.

[23] Gillespie, T. D. (1992). Fundamentals of Vehicle Dynamics, Society of Automotive Engineers Warrendale, PA.

[24] Gordon, T., Howell, M., and Brandao, F. (2003). "Integrated control methodologies for road vehicles." Vehicle System Dynamics, 40(1-3), 157-190.

[25] Gordon, T. J., and Magnuski, N. "Modeling Normal Driving as A Collision Avoidance Process." Proceedings of AVEC 2006.

[26] Gordon, T. J., and Best, M. C. (2006). "On the synthesis of driver inputs for the simulation of closed-loop handling manoeuvres." Int. J. of Vehicle Design, 40(13), 52-76.

[27] Gustafsson, F. (1997). "Slip-based tire-road friction estimation." Automatica, 33(6), 1087-1099.

[28] Hac, A., and Bodie, M. O. (2002). "Improvements in vehicle handling through integrated control of chassis systems." Int. J. of Vehicle Design, 29(1-2), 23-50.

[29] Hac, A., and Dickinson, J. E. (2006). "Collision avoidance with active steering and braking." US Patent 7016783.

[30] Hac, A. "Control of Brake- and Steer-by-Wire Systems During Brake Actuator Failure." SAE Technical Paper No 2006-01-0923.

[31] Hac, A., Bedner, E., and Fulk, D. "Exploring the Trade-Off of Handling Stability and Responsiveness with Advanced Control Systems." SAE Technical Paper No 2007-01-0812 
[32] Hattori, Y., Ono, E., and Hosoe, S. (2006). "Optimum vehicle trajectory control for obstacle avoidance problem." IEEE/ASME Transactions on Mechatronics, 11(5), 507-512.

[33] Hilgert, J., Hirsch, K., Bertram, T., and Hiller, M. "Emergency path planning for autonomous vehicles using elastic band theory." Proc. 2003 IEEE/ASME International Conf. on Advanced Intelligent Mechatronics.

[34] Hirano, Y., Harada, H., Ono, E., and Takanami, K. "Development of An Integrated System of 4ws and 4wd By H Infinity Control." SAE. J. 930267 pp. 7986.

[35] Horiuchi, S., Okada, K., and Nohtomi, S. (1999). "Improvement of vehicle handling by nonlinear integrated control of four wheel steering and four wheel torque." JSAE Review, 20(4), 459-464.

[36] Huh, K., Hong, D., Yoon, P., Kang, H.-J., and Hwang, I. "Robust Wheel Slip Control Brake by wire Systems." SAE Technical Paper No 2005-01-1584.

[37] iSight. (2004). User and Reference Manuals Version 9.0, Engineous Software Inc., Cary, NC USA.

[38] J. Yi, S. S., Howell, A., Horowitz, R., Tomizuka, M., Hedrick, J. K., and Alvarez, L. "Development and Implementation of a Vehicle-Centered Fault Diagnostic and Management System for the Extended PATH-AHS Architecture: Part I." PATH, Berkeley, CA, Rep. UCB-ITS-PRR-2002-34.

[39] Keviczky, T., and Balas, G. J. (2005). "Flight test of a receding horizon controller for autonomous UAV guidance." American Control Conference, 2005. Proceedings of the 2005, 3518-3523.

[40] Kiencke, U., and Nielsen, L. (2005). Automotive Control Systems, SpringerVerlag, New York, NY.

[41] Kitajima, K., and Peng, H. "H infinity control for integrated side-slip, roll and yaw controls for ground vehicles." Proceedings of AVEC 2000.

[42] Knoll, P. M. "Predictive Safety Systems: Convenience - Collision Mitigation Collision Avoidance." SAE Technical Paper No 2006-21-0082.

[43] Koehn, P., Eckrich, M., Smakman, H., and Schaffert, A. "Integrated Chassis Management: Introduction into BMW's Approach to ICM." SAE Technical Paper No 2006-01-1219 522.

[44] Komatsu, A., Gordon, T., and Best, M. (2000). "4WS Control of Handling Dynamics Using a Linear Optimal Reference Model." Proceedings of the Fifth International Symposium on Advanced Vehicle Control (AVEC2000), Ann Arbor, USA, 253-260.

[45] Kothare, M. V., Mettler, B., Morari, M., Bendotti, P., and Falinower, C.-M. (2000). "Level control in the steam generator of a nuclear power plant." IEEE Transactions on Control Systems Technology, 8(1), 55-69.

[46] Kou, Y., Peng, H., and Jung, D. "Worst-Case Evaluation for Integrated chassis control systems." IAVSD 20th Symposium: Dynamics of Vehicles on Roads and Tracks, Berkeley, CA, August 13-17, 2007. 
[47] Lim, E. H. M., and Hedrick, J. K. "Lateral and longitudinal vehicle control coupling for automated vehicle operation." San Diego, CA, USA, 3676-3680.

[48] Limpert, R. (1999). Brake design and safety, Society of Automotive Engineers Warrendale, $\mathrm{Pa}$.

[49] Liu, C.-S., and Peng, H. (1996). "Road friction coefficient estimation for vehicle path prediction." Vehicle System Dynamics, 25(Suppl), 413-425.

[50] Ma, W. H., and Peng, H. (1999). "Worst-Case Vehicle Evaluation MethodologyExamples on Truck Rollover/Jackknifing and Active Yaw Control Systems." Vehicle System Dynamics, 32(4), 389-408.

[51] Macadam, C. C. (2003). "Understanding and Modeling the Human Driver." Vehicle System Dynamics, 40(1), 101-134.

[52] Maciejowski, J. M. (2002). Predictive Control: With Constraints, Prentice Hall, UK.

[53] Mai, R., Bergholz, R., Lissel, E., and Van Meel, F. (2000). "Automatic emergency braking method and arrangement." U.S. Patent 6084508.

[54] Mastinu, G., Babbel, E., Lugner, P., Margolis, D., Mittermayr, P., and Richter, B. (1994). "Integrated controls of lateral vehicle dynamics." Vehicle System Dynamics, 23(SUPPL), 358-377.

[55] Mayne, D. Q., Rawlings, J. B., Rao, C. V., and Scokaert, P. O. M. (2000). "Constrained model predictive control: Stability and optimality." Automatica, 36(6), 789-814.

[56] Mechanical Simulation. (2004). CarSim Reference Manual Version 6, Mechanical Simulation Corporation, Ann Arbor, MI USA.

[57] Milliken, D. L., and Milliken, W. F. (2003). Race Car Vehicle Dynamics: Problems, Answers, and Experiments, SAE International.

[58] Mokhiamar, O., and Abe, M. (2002). "Active wheel steering and yaw moment control combination to maximize stability as well as vehicle responsiveness during quick lane change for active vehicle handling safety." Proceedings of the Institution of Mechanical Engineers, Part D: Journal of Automobile Engineering, 216(2), 115-124.

[59] Mokhiamar, O., and Abe, M. (2005). "Simultaneous Optimal Distribution of Lateral and Longitudinal Tire Forces for the Model Following Control." Journal of Dynamic Systems, Measurement, and Control, 126, 753.

[60] Morari, M., and Lee, J. H. (1999). "Model predictive control: past, present and future." Computers and Chemical Engineering, 23(4-5), 667-682.

[61] Nagai, M., Yamanaka, S., and Hirano, Y. (1999). "Integrated control of active rear wheel steering and yaw moment control using braking forces." JSME International Journal, Series C, 42(2), 301-308.

[62] NHTSA. (2007). FMVSS No. 126 Electronic Stability Control Systems, NHTSA Final Regulatory Impact Analysis. 
[63] Nwagboso, C. O., Ouyang, X., and Morgan, C. (2002). "Development of neuralnetwork control of steer-by-wire system for intelligent vehicles." Heavy Vehicle Systems, 9(1), 1-26.

[64] Ohue, K., Yamada, Y., Uozumi, S., Tokoro, S., Hattori, A., and Hayashi, T. "Development of a New Pre-Crash Safety System." SAE Technical Paper No 2006-01-1461 812.

[65] Pacejka, H. B. (2002). Tire and Vehicle Dynamics, SAE.

[66] Park, T. J., Han, C. S., and Lee, S. H. (2005). "Development of the electronic control unit for the rack-actuating steer-by-wire using the hardware-in-the-loop simulation system." Mechatronics, 15(8), 899-918.

[67] Polak, E., and Yang, T. H. (1993a). "Moving horizon control of linear systems with input saturation and plant uncertainty. I: Robustness." Int. J. of Control, 58(3), 613-638.

[68] Polak, E., and Yang, T. H. (1993b). "Moving horizon control of linear systems with input saturation and plant uncertainty. II: Disturbance rejection and tracking." Int. J. of Control, 58(3), 639-663.

[69] Prakah-Asante, K. O., Rao, M. K., and Strumolo, G. S. "Obstacle State Estimation For Imminent Crash Prediction \& Countermeasure Deployment DecisionMaking." SAE Technical Paper No 2003-01-2261.

[70] Prett, D. M., and García, C. E. (1988). Fundamental Process Control, Butterworths Boston.

[71] Qin, S. J., and Badgwell, T. A. (1997). "An overview of industrial model predictive control technology." Fifth International Conference on Chemical Process Control, 93, 232-256.

[72] Qin, S. J., and Badgwell, T. A. (2000). "An overview of nonlinear model predictive control applications." Nonlinear Predictive Control, 369-393.

[73] Qin, S. J., and Badgwell, T. A. (2003). "A survey of industrial model predictive control technology." Control Engineering Practice, 11(7), 733-764.

[74] Rajamani, R., Howell, A. S., Chen, C., Hedrick, J. K., and Tomizuka, M. (2001). "A Complete Fault Diagnostic System for Automated Vehicles Operating in a Platoon." IEEE TRANSACTIONS ON CONTROL SYSTEMS TECHNOLOGY, 9(4), 553.

[75] Ray, L. R. (1997). "Nonlinear tire force estimation and road friction identification: simulation and experiments." Automatica, 33(10), 1819-33.

[76] Rodic, A. D., and Vukobratovic, M. K. (1999). "Contribution to the integrated control synthesis of road vehicles." Control Systems Technology, IEEE Transactions on, 7(1), 64-78.

[77] Ryu, J., Rossetter, E. J., and Gerdes, J. C. "Vehicle Sideslip and Roll Parameter Estimation Using GPS." Proc. AVEC 2002 6th Int. Symp. Advanced Vehicle Control, Hiroshima, Japan. 
[78] Satria, M., and Best, M. "Comparison between kalman filter and robust filter for vehicle handling dynamics state estimation." SAE Technical Paper No 2002-011185.

[79] Schuette, H., and Waeltermann, P. "Hardware-In-The-Loop Testing of Vehicle Dynamics Controllers-A Technical Survey." SAE Technical Paper No 2005-011660.

[80] Seiler, P., Song, B., and Hedrick, J. K. "Development of a collision avoidance system." Detroit, MI, USA, 97-103.

[81] Shim, T., Chang, S., and Lee, S. (2007). "Investigation of Sliding Surface Design on the Performance of Sliding Mode Controller in Antilock Braking Systems." IEEE Transactions on Vehicular Technology (accepted for publication, 2007).

[82] Sledge Jr., N. H., and Marshek, K. M. "Development and Validation of An Optimized Emergency Lane-Change Trajectory." SAE Technical Paper No 980231.

[83] Slotine, J. J. E., and Li, W. (1991). Applied nonlinear control, Prentice Hall Englewood Cliffs, NJ.

[84] Song, B., Hedrick, J. K., and Howell, A. (2003). "Fault Tolerant Control and Classification for Longitudinal Vehicle Control." Journal of Dynamic Systems, Measurement, and Control, 125, 320.

[85] Sorniotti, A. "Hardware in the Loop for Braking Systems With Anti-Lock Braking System Electronic Stability Program." SAE Technical Paper No 2004-012062.

[86] Sorniotti, A., D'Alfio, N., Galvagno, E., Morgando, A., and Amisano, F. "Hardware-In-the-Loop Testing of Automotive Control Systems." SAE Technical Paper No 2006-01-1962

[87] Stence, R. "Digital By-Wire Replaces Mechanical Systems in Cars." $S A E$ Technical Paper No2004-01-2926.

[88] Takahashi, A., and Asanuma, N. "Introduction of Honda ASV-2(advanced safety vehicle-phase 2)." Dearbon, MI, USA, 694-701.

[89] Tanaka, H., Inoue, H., and Iwata, H. "Development of a Vehicle Integrated Control System." Proceedings of the XXIV FISITA Congress, June 1992, London, pp. 63-72.

[90] The MathWorks. (2005). Simulink User's Manual (Version 6.2), The Mathworks Inc., Natick, MA USA.

[91] The MathWorks. (2006a). Optimization Toolbox (Version 3), The Mathworks Inc., Natick, MA USA.

[92] The MathWorks. (2006b). Real-Time Winddows Target for Use with Real-Time Workshop (Version 2), The Mathworks Inc., Natick, MA USA.

[93] Tøndel, P., and Johansen, T. "Lateral vehicle stabilization using constrained nonlinear control." Proc. European Contr. Conf., Cambridge. 
[94] Tøndel, P., and Johansen, T. A. (2005). "Control allocation for yaw stabilization in automotive vehicles using multiparametric nonlinear programming." American Control Conference, 2005. Proceedings of the 2005, 453-458.

[95] Verschuren, R., and Duringhof, H.-M. "Design of a Steer-by-Wire Prototype." SAE Technical Paper No 2006-01-1497.

[96] Wang, Y. Q., and Nagai, M. (1999). "Improvement of Vehicle Handling and Stability by an Integrated Four-Wheel-Steer and Yaw Moment Control System." Progr. Technol, 77, 173-182.

[97] Wilkin, M. A., Crolla, D. C., and Levesley, M. C. "Design of a robust tyre force estimator using an extended kalman filter." SAE Technical Paper No 2005-010402 .

[98] Wu, D., Zhang, Q., Reid, J. F., Qiu, H., and Benson, E. R. (1998 ). "Model Recognition and Simulation of an E/H Steering Controller on Off-Road Equipment." ASME Fluid Power Systems and Technology 1998, 55-60.

[99] Yang, T. H., and Polak, E. (1993). "Moving horizon control of nonlinear systems with input saturation, disturbances and plant uncertainty." Int. J. of Control, 58(4), 875-903.

[100] Yao, Y. "Vehicle Steer-by-Wire System Control." SAE Technical Paper No 200601-1175.

[101] You, S. S., and Chai, Y. H. (1999). "Multi-objective control synthesis: An application to 4WS passenger vehicles." Mechatronics, 9(4), 363-390.

[102] Zeyada, Y. F., ElBeheiry, E. M., and ElArabi, M. E. (2000). "Fault Diagnosis and Mu-estimation for Integrated Chassis Control of Vehicles in Emergency Maneuvers." J. Eng. Appl. Sci. Cairo, 47, 665-682.

[103] Zhang, Y., Antonsson, E. K., and Grote, K. "A new threat assessment measure for collision avoidance systems." Proceedings. 2006 IEEE on Intelligent Transportation Systems, 968 - 975 